\title{
APPRENTISSAGE COLLABORATIF À DISTANCE
}

\section{POUR COMPRENDRE} ET CONCEVOIR LES ENVIRONNEMENTS D'APPRENTISSAGE VIRTUELS 




\section{APPRENTISSAGE \\ COLLABORATIF \\ À DISTANCE}




\section{PRESSES DE L'UNIVERSITÉ DU QUÉBEC}

Le Delta I, 2875, boulevard Laurier, bureau 450

Sainte-Foy (Québec) G1V 2M2

Téléphone: (418) 657-4399 • Télécopieur : (418) 657-2096

Courriel: puq@puq.uquebec.ca • Internet :www.puq.uquebec.ca

Distribution :

\section{CANADA et autres pays}

DISTRIBUTION DE LIVRES UNIVERS S.E.N.C.

845, rue Marie-Victorin, Saint-Nicolas (Québec) G7A 3S8

Téléphone : (418) 831-7474/1-800-859-7474 • Télécopieur : (418) 831-4021

\section{FRANCE}

DIFFUSION DE L'ÉDITION QUÉBÉCOISE

30, rue Gay-Lussac, 75005 Paris, France

Téléphone: 33143544902

Télécopieur: 33143543915

\section{SUISSE}

GM DIFFUSION SA

Rue d'Etraz 2, CH-1027 Lonay, Suisse

Téléphone : 0218032626

Télécopieur : 0218032629
DANGER

IE

PHOTOCOPILLAGE
TUE LE LIVRE
La Loi sur le droit d'auteur interdit la reproduction des œuvres sans autorisation des titulaires de droits. Or, la photocopie non autorisée - le «photocopillage »s'est généralisée, provoquant une baisse des ventes de livres et compromettant la rédaction et la production de nouveaux ouvrages par des professionnels. L'objet du logo apparaissant ci-contre est d'alerter le lecteur sur la menace que représente pour l'avenir de l'écrit le développement massif du «photocopillage». 


\section{APPRENTISSAGE COLLABORATIF À DISTANCE}

POUR COMPRENDRE ET CONCEVOIR LES ENVIRONNEMENTS D'APPRENTISSAGE VIRTUELS 
Henri, France

Apprentissage collaboratif à distance

Comprend des réf. bibliogr.

Sommaire: [1] Pour comprendre et concevoir les environnements d'apprentissage virtuels.

ISBN 2-7605-1094-8 (v. 1)

1. Enseignement à distance - Innovations. 2. Apprentissage - Travail en équipe.

3. Technologie éducative. 4. Télécommunications en éducation. 5. Internet en éducation. I. Lundgren-Cayrol, Karin, 1946- ～. II. Titre.

LC5800.H46 $2000 \quad$ 371.3'58 C00-941958-6

Nous reconnaissons l'aide financière du gouvernement du Canada par l'entremise du Programme d'aide au développement de l'industrie de l'édition (PADIÉ) pour nos activités d'édition.

Mise en pages: CARACTÉRA PROdUCtION GRAPHIQUE INC.

Couverture: Deschamps Design

123456789 PUQ 2001987654321

Tous droits de reproduction, de traduction et d'adaptation réservés (C) 2001 Presses de l’Université du Québec

Dépôt légal - $1^{\text {er }}$ trimestre 2001

Bibliothèque nationale du Québec / Bibliothèque nationale du Canada Imprimé au Canada 


\section{Avant-propos}

C

et ouvrage est l'occasion de partager le résultat des travaux que nous menons depuis quelques années pour mieux comprendre les pratiques nouvelles qui, en formation à distance, émergent sous la poussée des nouvelles technologies. Impossible ici d'aborder cette question dans toute sa complexité et toute son étendue. Notre étude se veut une humble contribution aux efforts de recherche sur ce que peut être l'apprentissage à distance, à l'aide des technologies.

Nous avons voulu décrire la nature et le fonctionnement de l'apprentissage à distance, en réseau, par la communication avec les pairs. Avec le plus de rigueur possible, nous avons étudié le phénomène de l'apprentissage collaboratif en mode virtuel pour en dégager le sens et les fondements théoriques. En nous appuyant sur notre formation en technologie éducative, nous avons tenté de percer les mécanismes de la collaboration et d'élaborer un cadre de référence pour ceux et celles qui doivent concevoir et développer des environnements d'apprentissage virtuels. Notre travail adopte donc le point de vue de la conception pédagogique. La conception pédagogique est ici synonyme de design pédagogique, mais se distingue de l'ingénierie pédagogique, un domaine plus vaste recouvrant la planification, la conception, la réalisation, l'implantation et la diffusion de systèmes d'apprentissage.

Par notre propos, nous exprimons notre conviction qu'en formation à distance la présence existe: présence intime, à soi, aux autres et à la connaissance. À ceux et celles qui croient que l'apprentissage est un acte de proximité, nous voulons dire que le partage intellectuel et humain est possible même à distance et 
viii

qu'il peut être l'occasion de vivre des expériences de croissance engageantes envers soi-même et envers les autres. Nous sommes convaincues que la formation à distance peut être émancipatrice, en raison de la distance, justement. Il ne s'agit pas d'opposer formation présentielle et formation à distance. Car, comme le dit Michel Bernard, il y a de la distance en présentiel et du présentiel à distance. Ce sont les modalités et les fondements de la présence qui diffèrent dans l'un et l'autre cas. 
À tous nos collègues $d u$ Centre de recherche LICEF qui, de près ou de loin, ont participé à notre recherche. 



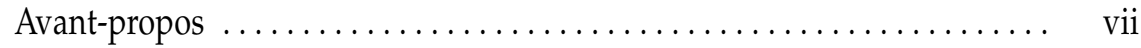

Remerciements ................................... ix

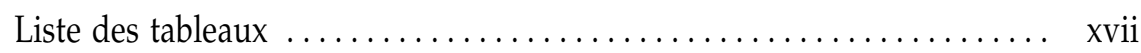

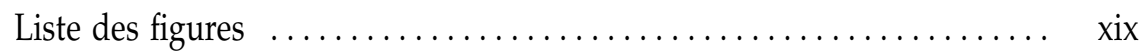

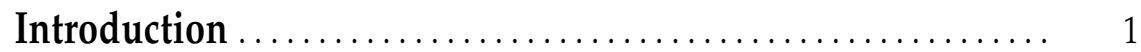

La poussée des technologies $\ldots \ldots \ldots \ldots \ldots \ldots \ldots \ldots \ldots \ldots \ldots \ldots . \ldots \ldots \ldots \ldots$

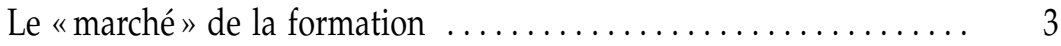

Formation et distance $\ldots \ldots \ldots \ldots \ldots \ldots \ldots \ldots \ldots \ldots \ldots \ldots, 4$

L'apprentissage collaboratif $\ldots \ldots \ldots \ldots \ldots \ldots \ldots \ldots \ldots \ldots \ldots \ldots \ldots$

Organisation de l'ouvrage $\ldots \ldots \ldots \ldots \ldots \ldots \ldots \ldots \ldots \ldots \ldots . \ldots \ldots$

Chapitre 1

Vers une définition de l'apprentissage collaboratif ......... 9

L'apprentissage selon les constructivistes $\ldots \ldots \ldots \ldots \ldots \ldots \ldots \ldots . \quad 10$

Les connaissances: des entités subjectives en quête de viabilité .... 11

Pour une pédagogie de collaboration et de coopération ......... 12

Pour une pédagogie réflexive $\ldots \ldots \ldots \ldots \ldots \ldots \ldots \ldots \ldots . \ldots \ldots$

Contribution du constructivisme à l'apprentissage collaboratif ..... 14

L'apprentissage selon les psychocognitivistes $\ldots \ldots \ldots \ldots \ldots \ldots \ldots \ldots . \ldots \ldots$

Pour une pédagogie de structuration $\ldots \ldots \ldots \ldots \ldots \ldots \ldots \ldots \ldots, 15$

Contribution des psychocognitivistes

à l'apprentissage collaboratif $\ldots \ldots \ldots \ldots \ldots \ldots \ldots \ldots \ldots \ldots, 16$

L'apprentissage selon les sociocognitivistes $\ldots \ldots \ldots \ldots \ldots \ldots \ldots \ldots$

Pour une pédagogie marquée par l'ancrage social et culturel $\ldots \ldots . \quad 17$

Contribution des sociocognitivistes à l'apprentissage collaboratif ... 18 
La théorie de la flexibilité cognitive $\ldots \ldots \ldots \ldots \ldots \ldots \ldots \ldots \ldots . \ldots . \ldots . \ldots 19$

Les domaines complexes, peu ou mal structurés ............. 19

Connaissances initiales et connaissances avancées $\ldots . \ldots \ldots \ldots .20$

Flexibilité cognitive et apprentissage de contenus complexes,

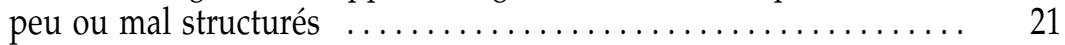

Contribution de la flexibilité cognitive

à l'apprentissage collaboratif ....................... 22

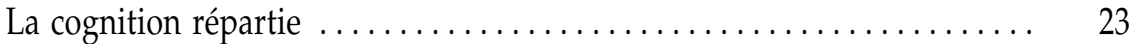

Contribution de la cognition répartie

à l'apprentissage collaboratif $\ldots \ldots \ldots \ldots \ldots \ldots \ldots \ldots \ldots \ldots, 26$

Les principes andragogiques $\ldots \ldots \ldots \ldots \ldots \ldots \ldots \ldots \ldots \ldots . \ldots \ldots$

Contribution de l'andragogie à l'apprentissage collaboratif ....... 28

La distinction entre l'apprentissage coopératif

et l'apprentissage collaboratif $\ldots \ldots \ldots \ldots \ldots \ldots \ldots \ldots \ldots \ldots, 28$

Maturité, contrôle et autonomie ....................... 30

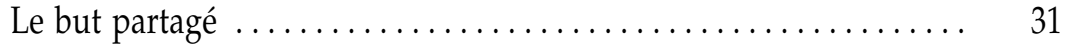

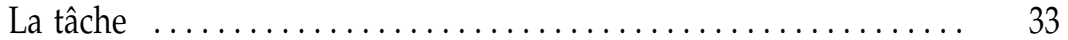

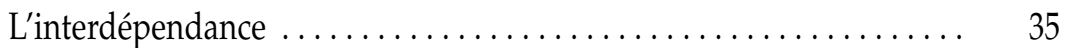

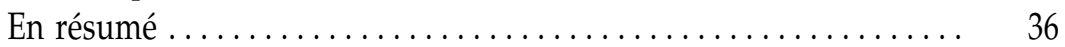

Vers une définition de l'apprentissage collaboratif ............ 42

\section{Chapitre 2}

\section{Environnements d'apprentissage virtuels,} communication et collaboration ....................... 45

Les environnements d'apprentissage virtuels $\ldots \ldots \ldots \ldots \ldots \ldots \ldots . . \ldots$

La notion d'environnement d'apprentissage virtuel ........... 48

La construction d'environnements d'apprentissage $\ldots \ldots \ldots \ldots . \quad 50$

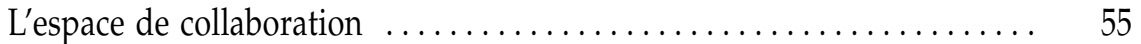

L'espace privé: un centre de réalisation

de la tâche d'apprentissage $\ldots \ldots \ldots \ldots \ldots \ldots \ldots \ldots \ldots \ldots . \ldots \ldots \ldots$

L'espace commun: un centre de ressources $\ldots \ldots \ldots \ldots \ldots \ldots \ldots . \quad 58$

L'espace de communication de groupe:

un centre de négociation et de validation

des connaissances .............................. 59

Communication, collaboration et apprentissage $\ldots \ldots \ldots \ldots \ldots \ldots \ldots .60$

La conversation au cœur de la collaboration ............... 60 
La conversation comme modèle d'apprentissage $\ldots \ldots \ldots \ldots \ldots .61$

La communication de groupe synchrone ou asynchrone $\ldots \ldots \ldots .63$

L'interaction, écrite et asynchrone $\ldots \ldots \ldots \ldots \ldots \ldots \ldots \ldots \ldots \ldots \ldots$

Le forum électronique et autres technologies

de communication asynchrone $\ldots \ldots \ldots \ldots \ldots \ldots \ldots \ldots . \ldots . \ldots . \ldots . \ldots 6$

Un mode inédit de communication humaine $\ldots \ldots \ldots \ldots \ldots \ldots .69$

Les limites cognitives $\ldots \ldots \ldots \ldots \ldots \ldots \ldots \ldots \ldots \ldots \ldots \ldots \ldots$

Limite de la présence sociale $\ldots \ldots \ldots \ldots \ldots \ldots \ldots \ldots . \ldots \ldots$

Limite du processus de développement de la pensée $\ldots \ldots \ldots \quad 72$

Limite $d u$ processus réflexif $\ldots \ldots \ldots \ldots \ldots \ldots \ldots \ldots, \quad 73$

Limites à la structuration des échanges $\ldots \ldots \ldots \ldots \ldots \ldots \quad 74$

Limites du langage verbal et de la conversation .......... 75

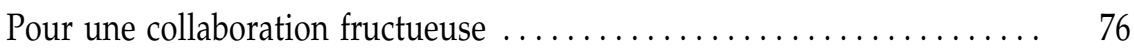

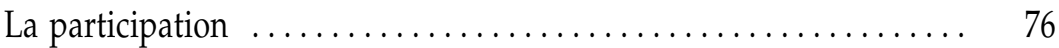

Établir les règles du jeu et développer une perception

positive de la collaboration ...................... 77

La motivation ................................... $\quad 78$

La motivation extrinsèque $\ldots \ldots \ldots \ldots \ldots \ldots \ldots \ldots \ldots \ldots, \quad 78$

La motivation intrinsèque $\ldots \ldots \ldots \ldots \ldots \ldots \ldots \ldots \ldots \ldots$

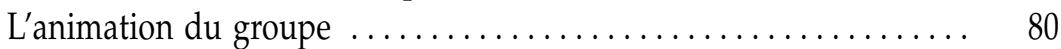

La cohésion et la productivité du groupe $\ldots \ldots \ldots \ldots \ldots \ldots \ldots . \quad 82$

Pour structurer l'espace de communication $\ldots \ldots \ldots \ldots \ldots \ldots \ldots \ldots . \quad 84$

Forums et lieux de construction collective des connaissances ..... 85

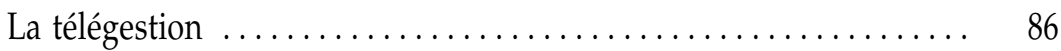

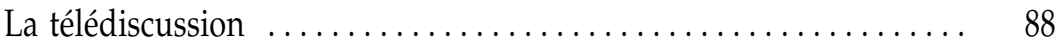

Le télétravail ............................... 90

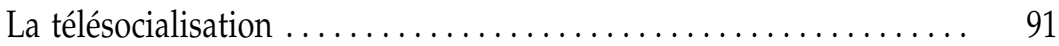

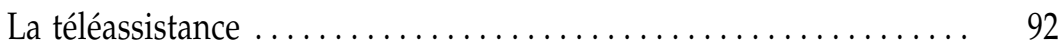

Pour une structure souple $\ldots \ldots \ldots \ldots \ldots \ldots \ldots \ldots \ldots . \ldots 4$

\section{Chapitre 3}

Modèle et ressources de collaboration à distance .......... 95

Le groupe et la collaboration $\ldots \ldots \ldots \ldots \ldots \ldots \ldots \ldots \ldots \ldots \ldots \ldots$

Qu'est-ce qu'un groupe ? .......................... 96

Un groupe est plus qu'un regroupement $\ldots \ldots \ldots \ldots \ldots \ldots .97$

La cible commune des groupes d'apprentissage .......... 98 
Les bases d'un modèle de collaboration pour les groupes d'apprenants ..................... 98

Trois composantes d'un modèle de collaboration .......... 99

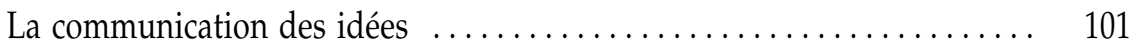

Exprimer des idées afin de les partager $\ldots \ldots \ldots \ldots \ldots \ldots \ldots . \ldots 102$

Ressources de l'environnement technologique ........... 103

Établir des liens entre les idées pour faire émerger des idées nouvelles .................................... 104

Ressources de l'environnement technologique ........... 104

Structurer les idées pour leur donner un sens ............. 105

Ressources de l'environnement technologique .......... 107

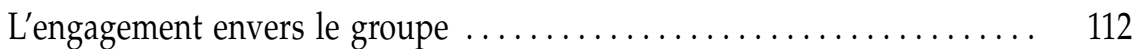

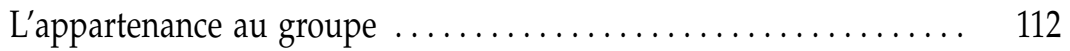

Ressources de l'environnement technologique .......... 115

Les espaces de travail virtuels $\ldots \ldots \ldots \ldots \ldots \ldots \ldots \ldots . \ldots 116$

La cohésion et la productivité ...................... 117

La cohésion .............................. 117

La productivité .............................. 119

Cohésion et productivité ....................... 120

Ressources de l'environnement technologique ............ 121

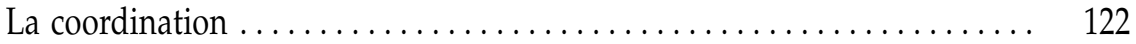

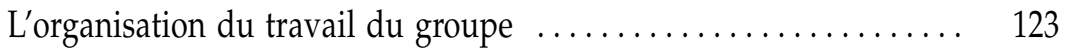

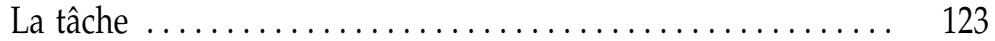

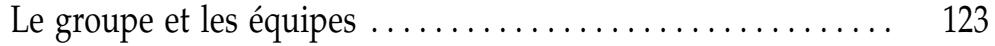

L'animation ................................ 125

Ressources de l'environnement technologique ............. 126

Pour le soutien à la tâche ...................... 126

La constitution du groupe et des équipes $\ldots \ldots \ldots \ldots \ldots \ldots . \ldots 126$

L'animation ................................ 126

La structuration de l'espace de communication ............. 127

Ressources de l'environnement technologique .......... 128

Tableau synthèse des ressources de collaboration $\ldots \ldots \ldots \ldots \ldots \ldots . . \ldots$ 


\section{Chapitre 4}

La dynamique de l'apprentissage collaboratif ........... 131

La dynamique cognitive de l'apprentissage collaboratif $\ldots \ldots \ldots \ldots . . \ldots 132$

Les quatre principaux acteurs de l'interaction cognitive ........ 134

Le modèle de connaissances de l'expert ............... 134

L'apprenant .............................. 134

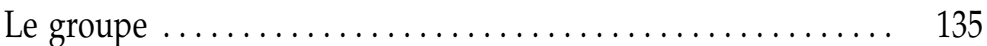

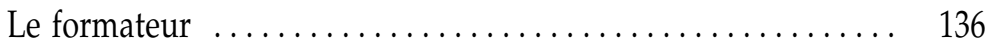

La démarche de collaboration ......................... 139

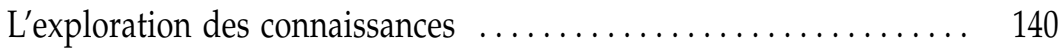

L'élaboration du modèle de connaissances .................. 141

L'évaluation des apprentissages et du processus de collaboration ... . 142

Résumé de la démarche de collaboration $\ldots \ldots \ldots \ldots \ldots \ldots . . \ldots 143$

Les habiletés de l'apprenant ........................... 147

Les habiletés cognitives $\ldots \ldots \ldots \ldots \ldots \ldots \ldots \ldots \ldots \ldots . \ldots \ldots$

Apprendre à collaborer ............................ 152

Négociation du modèle de collaboration par le groupe ...... 153

Le soutien à la construction des connaissances .................. 156

Les rôles $d u$ formateur $\ldots \ldots \ldots \ldots \ldots \ldots \ldots \ldots \ldots \ldots . \ldots \ldots$

Les interventions spécifiques aux différentes phases

de la collaboration ................................. 157

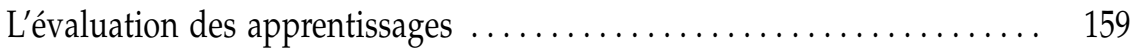

L'évaluation des apprentissages cognitifs $\ldots \ldots \ldots \ldots \ldots \ldots \ldots . \ldots 159$

Comprendre et évaluer les modèles mentaux des apprenants .... 160

Qu'est-ce qu'un modèle mental et comment l'évaluer? ....... 160

L'évaluation de la démarche de collaboration ............... 162

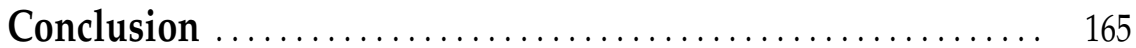

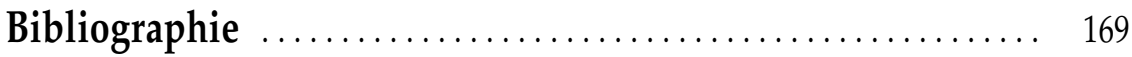

Index des auteurs cités $\ldots \ldots \ldots \ldots \ldots \ldots \ldots \ldots \ldots \ldots \ldots$ 

1.1 Interdépendance en coopération et en collaboration $\ldots \ldots \ldots \ldots \ldots \quad 37$

1.2 Comparaison des démarches coopérative et collaborative ....... 39

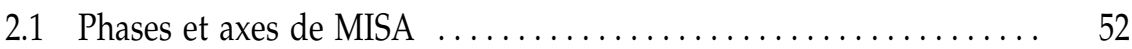

2.2 Le forum électronique $\ldots \ldots \ldots \ldots \ldots \ldots \ldots \ldots \ldots \ldots \ldots \ldots$

2.3 Partage du rôle de modérateur
entre le formateur et les apprenants $\ldots \ldots \ldots \ldots \ldots \ldots \ldots \ldots \ldots \ldots$

3.1 Dynamique de la communication

des idées et activités cognitives correspondantes ........... 102

3.2 Jeu de rôles pour la production d'une carte conceptuelle: stratégie pour la structuration des idées $\ldots \ldots \ldots \ldots \ldots \ldots \ldots .106$

3.3 Prises de conscience associées à l'apprentissage collaboratif ...... 113

3.4 Interventions pour stimuler le sentiment d'appartenance ........ 114

3.5 Exemple de comportements indiquant une bonne cohésion du groupe $\ldots \ldots \ldots \ldots \ldots \ldots \ldots \ldots \ldots . . \ldots \ldots$

3.6 Exemple de résultats de l'évaluation

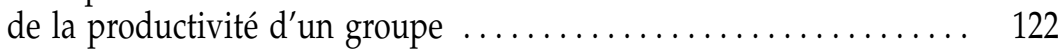

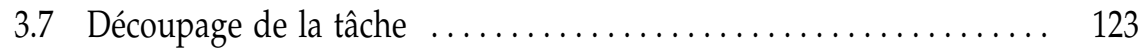

3.8 Problématique de la constitution et de la composition d'un groupe .................... 124

3.9 Animation des forums électroniques $\ldots \ldots \ldots \ldots \ldots \ldots \ldots \ldots . \ldots \ldots$

3.10 Espèces de forums en rapport avec la tâche, la taille $d u$ groupe et l'animation $\ldots \ldots \ldots \ldots \ldots \ldots \ldots \ldots, 128$

3.11 Synthèse des ressources de collaboration $\ldots . \ldots \ldots \ldots \ldots \ldots \ldots . . \ldots . \ldots 129$

4.1 Processus et résultantes des trois phases de la démarche collaborative ...................... 144 
4.2 Les composantes du modèle de collaboration et les phases de la démarche collaborative ...

4.3 Les habiletés de la pensée réflexive sollicitées aux trois phases de la collaboration .................... 150

4.4 Processus continu de négociation du modèle de collaboration ..... 154

4.5 Les rôles et les interventions du formateur pour le soutien à l'acquisition des connaissances ............ 157

4.6 Critères pour évaluer les modèles mentaux ............... 162

4.7 Les critères d'évaluation $\ldots \ldots \ldots \ldots \ldots \ldots \ldots \ldots \ldots \ldots \ldots \ldots$ 


\section{Liste des figures}

1.1 De la coopération à la collaboration:

un exercice de croissance vers l'autonomie $\ldots \ldots \ldots \ldots \ldots \ldots \ldots, 31$

1.2 Modalité de la tâche coopérative $\ldots \ldots \ldots \ldots \ldots \ldots \ldots \ldots \ldots . \ldots \ldots$

1.3 Modalité de la tâche collaborative $\ldots \ldots \ldots \ldots \ldots \ldots \ldots \ldots . . \ldots \ldots$

1.4 La tâche collaborative: la même pour tous et réalisée par tous .... 34

1.5 La tâche coopérative: un ensemble de sous-tâches différentes réalisées en équipe $\ldots \ldots \ldots \ldots \ldots \ldots \ldots \ldots \ldots \ldots \ldots \ldots \ldots \ldots, 34$

2.1 Acteurs et processus du campus virtuel $\ldots \ldots \ldots \ldots \ldots \ldots \ldots . \ldots \ldots$

2.2 Cinq espaces du campus virtuel $\ldots \ldots \ldots \ldots \ldots \ldots \ldots \ldots \ldots \ldots$

2.3 Espaces et outils de collaboration ...................... 56

2.4 L'aménagement des lieux d'échange, de travail

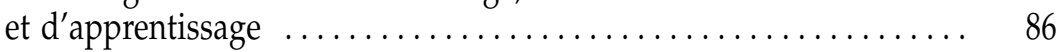

2.5 Lieux de télégestion $\ldots \ldots \ldots \ldots \ldots \ldots \ldots \ldots \ldots \ldots \ldots \ldots \ldots \ldots$

2.6 Lieux de la télédiscussion . ......................... 89

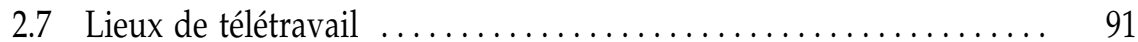

2.8 Lieux de télésocialisation $\ldots \ldots \ldots \ldots \ldots \ldots \ldots \ldots \ldots \ldots . \ldots . \ldots . \ldots . \ldots$

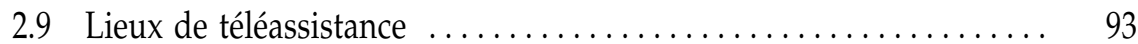

3.1 Composantes de base de la dynamique de collaboration ........ 100

3.2 Exemple de modèle de connaissances ..................... 108

3.3 Un exemple de carte conceptuelle et de liens hypertextes ........ 110

3.4 Interactions au sein d'un groupe d'apprenants $\ldots \ldots \ldots \ldots \ldots \ldots . . \ldots$

3.5 Exemples d'interfaces indiquant la présence des pairs .......... 117

3.6 Corrélation positive entre la cohésion

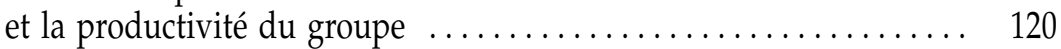

4.1 Apprendre: intégrer l'information dans un usage ........... 137 
4.2 La dynamique cognitive de l'apprentissage collaboratif $\ldots \ldots \ldots . \quad 138$

4.3 L'évolution de l'apprenant ........................... 155

4.4 Le formateur: de modérateur à animateur .............. 158 


\section{Introduction}

L

a technologie a toujours été présente à travers l'histoire. Par elle, l'homme n'a cessé d'inventer des moyens pour transformer et aménager ce qui l'entoure. Aujourd'hui, devant la fantastique évolution des nouvelles technologies de l'information et de la communication (NTIC) et leur pénétration à l'échelle planétaire, deux camps s'opposent (Bélanger, 1997). L'un, formé de technicistes, fait la promotion des NTIC et vante leurs bienfaits en annonçant une société de l'information, une société du savoir, où tout se fait au profit du citoyen. L'autre, formé de techno-sceptiques, voit dans la poussée des technologies la poursuite de l'organisation d'une société de marché qui tend à la mondialisation des modes de production et de consommation. En tant qu'éducateurs et formateurs, nous ne pouvons faire l'économie d'une réflexion pour tenter de cerner le rôle des nouveaux outils du savoir et de découvrir comment ils peuvent être utiles à l'apprentissage. Chacun imprégnera cet exercice de sa philosophie, de ses valeurs, de ses croyances, et chacun le fera aussi avec sa vision de l'éducation et de la formation. Pour notre part, nous considérons l'éducation comme une démarche vers l'épanouissement de l'individu et de sa collectivité et vers leur enrichissement culturel. Par son contenu et son organisation, la démarche éducative mise sur le développement de capacités généralistes grâce auxquelles l'apprenant façonne sa connaissance du monde. Tenant pour acquis que l'éducation, jamais achevée, est présente toute la vie durant, nous concevons la formation comme un projet, comme une œuvre que le formé poursuit avec une visée précise pour son plus grand accomplissement. La formation, pour nous, n'est donc pas synonyme de spécialisation et d'appropriation de connaissances strictement utilitaires. C'est avec cette vision que 
nous analysons les potentialités des NTIC et que nous avons voulu décrire des usages qui sont véritablement au service de l'épanouissement des êtres et de leur formation.

\section{LA POUSSÉE DES TECHNOLOGIES}

Les NTIC sont dites nouvelles parce qu'elles présentent et véhiculent l'information différemment, parce qu'elles permettent de communiquer autre chose, autrement. Elles surpassent ce que nous connaissions déjà en termes de multiplication, de reproduction, de distribution et de stockage d'information. À l'heure des NTIC, l'information est présente partout sous une diversité de formes. La communication humaine subit également de grands bouleversements. L'inédit de cette communication n'est pas de faire passer rapidement et efficacement un message d'un point à un autre. Ce qui est nouveau, c'est ce qui est communiqué ; c'est de communiquer autre chose, autrement. Cet autre chose, autrement, semble si attrayant, si envoûtant et si convaincant qu'on ne peut pas freiner la multiplication des communautés virtuelles. Ces groupes, innombrables, s'intéressent à tous les sujets imaginables et on les retrouve dans tous les secteurs de l'activité humaine. Des personnes, dans le cyberespace, vivent par ces communautés des expériences qu'elles décrivent comme uniques. Ce phénomène n'est pas absent du monde de l'éducation et de la formation. Des apprenants se rassemblent pour vivre dans le virtuel des expériences d'apprentissage différentes.

Dans un contexte où les NTIC s'imposent et nous sont imposées, et en raison des potentialités qu'on leur reconnaît, nous sommes inévitablement amenés à redéfinir l'activité éducative et de formation. Dans la même foulée, les établissements éducatifs et de formation, qui ont fondé leur existence sur la rareté de l'information et des connaissances et qui affrontent actuellement la concurrence des technologies, sont fortement remis en question (Bélanger, 1997). 


\section{LE «MARCHÉ» DE LA FORMATION}

Les NTIC ont créé un contexte économique et concurrentiel qui pousse à envisager la formation différemment. Les capacités accrues de démultiplication et donc d'industrialisation ont fait naître diverses industries: celles de la langue, de la culture, de la connaissance, de la formation. On parle aujourd'hui du marché de la formation. Dans ce contexte, il y a des produits, des vendeurs, mais aussi des acheteurs. Les établissements de formation, ayant été largement soutenus par les fonds publics et mis à l'abri des initiatives mercantiles, ont vu au cours des dernières années leurs ressources amputées. Pour maintenir leur activité, ils cherchent avidement des acheteurs; ils adoptent une logique de compétition pour accroître leur part de ce marché (Mason, 1998). Face à cette situation, on cherche en vain les fondements de la formation. Dans ce marché, on observe plutôt que les technologies sont devenues des gadgets, une sorte de plus-value qui fait «vendre» la formation. Elles ne sont pas envisagées comme de véritables outils de formation, comme des moyens au service de l'apprentissage. Nous est-il encore possible de proposer une autre vision des technologies, un autre paradigme?

De tout temps, l'éducation et la formation ont été influencées par les technologies. L'écriture, l'imprimé puis l'audiovisuel et la micro-informatique ont modifié la formation. Lorsqu'elles sont apparues, ces technologies ont fourni des moyens nouveaux de mise en mémoire et de circulation des connaissances. Aujourd'hui, à leur tour, les NTIC continuent de transformer le processus d'éducation et de formation. Avec des capacités décuplées, elles y sont présentes à titre de mémoire, de véhicule et de moyen de présentation de l'information, mais aussi à titre d'outils de communication humaine, de collaboration, d'assistance et de construction des connaissances. À cet égard, les travaux récents en sciences cognitives permettent de mieux appréhender l'usage des possibilités des technologies en éducation et en formation. Elles peuvent contribuer à reconstruire et à renouveler en profondeur la formation en "provoquant et déconstruisant l'existant» (Bernard, 1999). L'apport des possibilités des NTIC peut être déterminant pour le renouvellement de 
la formation, pour repenser l'activité de formation afin de se sortir d'une économie de marché et de s'orienter vers une économie sociale. Il nous faut alors œuvrer pour que les technologies soient utilisées en vue de donner à la personne en formation le rôle d'auteur de ses connaissances. Il faut que les acteurs de l'éducation et de la formation prennent le virage et s'engagent à parler désormais d'apprentissage et d'autonomie de l'apprenant.

\section{FORMATION ET DISTANCE}

La formation à distance est décrite le plus souvent comme un mode de formation économique qui utilise des technologies pour franchir la distance spatiotemporelle, améliorant ainsi l'accessibilité dans un idéal de démocratisation de l'éducation. En formation à distance, tout est mis en œuvre pour pallier l'absence qui est vue comme la plus grande faiblesse du concept. Et si, en formation à distance, l'absence n'était pas quelque chose à combler? Si la distance était le résultat d'un choix inhérent à la formation? Alors la distance ne serait plus réduite à un écart spatiotemporel; elle deviendrait une nécessité, une contribution à la spécificité et aux fondements de la formation.

Pour Bernard (1999), la distance est plurielle. «Il y a des distances insécurisantes, il y a des distances de sécurité. Il y a des distances génératrices de présence, il y a des distances provocatrices d'absences. Il y a, par les distances, des éloignements salvateurs, pertinents ou insupportables» (p. 35). Bernard propose d'envisager la distance, traitée généralement de façon trop restrictive, trop technique et trop organisationnelle, comme une dimension positive même si, comme la formation elle-même, elle exige plusieurs qualités chez la personne en formation: désir, volonté, énergie, ténacité et méthode personnelle. Mettre en valeur la distance comme inhérente à l'action de la personne pour produire sa propre formation, c'est miser sur l'autoformation. Cette mise en valeur de la distance ne devrait pas pour autant évacuer le groupe, la collégialité et la communauté qui font de la formation un processus de socialisation où les pairs et le 
formateur sont des partenaires. Pour Bernard, la mise à distance intègre la formation comme résultat de l'autoformation et de la coformation (p. 149).

La distance constitue donc un potentiel pour forger l'œuvre de formation. Elle n'en transforme pas la spécificité et les fondements; elle les maintient et peut même les renforcer. Toujours selon Bernard, la distance est à envisager suivant deux axes : celui des significations, qui fournit les fondements, et celui des situations, qui négocie avec les éléments de la réalité. Ce qui diffère dans les situations de formation à distance, ce sont les modalités, les outils et les méthodes utilisés pour permettre à l'apprenant de contrôler sa démarche d'autoformation et de s'engager dans un processus de coformation.

\section{L'APPRENTISSAGE COLLABORATIF}

On reproche souvent à la formation à distance d'être privée d'interactions, de ne pas permettre le dialogue et de se limiter à des situations d'apprentissage individuel. Les médias traditionnels ne rendent pas possibles les relations soutenues entre l'apprenant, le formateur et les pairs. Ni les logiciels d'enseignement assisté par ordinateur (EAO) ni les tutoriels intelligents les plus puissants ne peuvent simuler de manière intégrale le répertoire cognitif et social de l'humain ou l'accompagnement du formateur et la présence des pairs. Ce qui manque à la formation à distance depuis ses débuts, c'est la capacité de soutenir la communication humaine, cette composante de l'expérience d'apprentissage valorisante et significative qui ne peut être réduite. Avec les NTIC, on peut actuellement entrevoir une solution à ce problème. Il devient possible d'intégrer dans la formation à distance la relation humaine en continu, l'interaction et le dialogue soutenus, et cela, surtout par la communication asynchrone (Chambers, 1999). Désormais, il est plus facilement envisageable de parler de coformation, de communauté virtuelle d'apprentissage, de collaboration, d'interaction de groupe, de renforcement du caractère social de l'apprentissage, de valorisation du partage et, encore plus qu'avant, de développement de l'autonomie. 
Dans des environnements d'apprentissage virtuels, les apprenants peuvent travailler ensemble pour découvrir de nouveaux univers de connaissances, tout en gardant l'entière responsabilité de leur apprentissage et de leur autoformation. Dans ce contexte, le groupe joue un rôle actif de soutien. Il n'a pas la connotation de «classe" qui rassemble de manière quelque peu artificielle des apprenants qui sont spectateurs d'un enseignement. Le groupe a plutôt le sens de «cohorte » où des apprenants vivent ensemble un même événement et participent de manière volontaire à des activités communes (Miller, 1995). S'engager dans une démarche d'apprentissage collectif ne signifie pas s'en remettre au groupe pour apprendre. Chacun travaille activement, à sa manière, à la construction de ses propres connaissances. Au fil des échanges et des travaux de groupe, l'apprentissage se réalise par la discussion, le partage, la négociation et la validation des connaissances nouvellement construites. Ce processus qui, en lui-même, est un objet de formation suscite le développement d'habiletés de collaboration et la mise en œuvre de stratégies cognitives et métacognitives de haut niveau.

Les communautés d'apprenants peuvent désormais vivre à distance dans des environnements technologiques rendus accessibles par diverses infrastructures de réseaux qui les rejoignent et les réunissent afin qu'ils puissent travailler et apprendre en collaborant. Dans ces environnements, on utilise des médias plus ou moins interactifs de présentation de contenu; on donne accès à des ressources de toutes sortes incluant des outils et des espaces de collaboration. Mais ce ne sont pas les médias et les technologies qui créent les communautés d'apprenants, qui animent la communication de groupe, qui motivent à la participation, au partage et à l'entraide. C'est plutôt la philosophie et la pédagogie qui soustendent la conception de ces environnements qui induisent la naissance et le développement de la collaboration ; c'est notre capacité de donner aux apprenants le contrôle accru de leur apprentissage, notre habileté à créer des environnements assez riches pour répondre aux besoins de chacun et notre volonté de favoriser un réel dialogue entre l'apprenant, le groupe et le formateur. 
D’aucuns s'inquiètent de la validité des démarches pédagogiques fondées sur la collaboration et la négociation des apprentissages. Ils s'interrogent, ou ne reconnaissent pas aux apprenants la capacité de décider ce qu'ils doivent apprendre. Ils craignent de laisser l'ignorance guider l'apprentissage. Ils voient des risques à promouvoir la collaboration qui renforce le rôle d'apprenant. Sous la poussée des NTIC et le développement d'Internet, la culture de la collaboration se développe de plus en plus. Elle exerce des pressions qui ne peuvent être que salutaires pour le renouvellement de nos systèmes de formation. Elle oblige les «fournisseurs » de la formation à se préoccuper des besoins des apprenants plutôt qu'à s'intéresser uniquement à la transmission du canon préétabli de la connaissance (Mason, 1998).

Mais la démarche d'apprentissage collaboratif n'est pas universelle; elle n'est pas la seule façon d'apprendre et elle ne convient pas à toutes les situations de formation. Elle sera judicieusement choisie par le concepteur de la formation selon l'apprenant, le milieu, la culture, l'objet et les objectifs de formation. Si nous nous y intéressons, c'est parce qu'elle peut contribuer au développement de l'être et que, à un moment ou un autre, elle devrait faire partie de la formation. Nous nous y intéressons parce qu'il est désormais possible de concevoir, à distance, une formation complète, de la soutenir par des environnements technologiques pouvant permettre la collaboration, des environnements qui n'ont rien d'aliénant et où l'on vit une forme de présence, de proximité et d'émancipation.

\section{ORGANISATION DE L'OUVRAGE}

Dans le but de mieux comprendre les mécanismes de construction des connaissances dans une perspective d'auto- et de coformation, cet ouvrage analyse les fondements de l'apprentissage collaboratif, les modalités de son fonctionnement dans un environnement virtuel et les outils technologiques qui peuvent être utilisés pour le soutenir.

Dans le premier chapitre, nous étudions les assises théoriques de l'apprentissage collaboratif. Nous y établissons la distinction entre les démarches coopérative et collaborative avant de proposer 
une définition de l'apprentissage collaboratif. Nous abordons, dans le deuxième chapitre, le fonctionnement de la collaboration dans les environnements virtuels. Nous y présentons le forum électronique à la fois comme un lieu de collaboration et comme une technologie d'apprentissage. Dans le troisième chapitre, nous décrivons les ressources, humaines et technologiques, de l'environnement de collaboration. Nous mettons en lien des outils technologiques et le soutien à la démarche de collaboration. Nous décrivons, dans le quatrième chapitre, la dynamique cognitive de l'apprentissage collaboratif, les habiletés cognitives de l'apprenant de même que le rôle du formateur qui l'accompagne dans sa démarche. 


\section{Chapitre 1}

\section{Vers une définition de l'apprentissage collaboratif}

\section{工'}

apprentissage collaboratif est un construit dont les assises ont plusieurs sources. D'abord, il s'alimente aux valeurs du constructivisme et il s'appuie sur les théories cognitivistes pour expliquer les mécanismes de l'apprentissage. Ensuite, il trouve dans la théorie de la flexibilité cognitive, dans le concept de cognition répartie et dans l'andragogie les supports théoriques qui permettent d'établir des principes pour baliser la démarche d'apprentissage collaboratif.

Dans ce chapitre, nous étudions les diverses contributions qui servent de fondements à l'apprentissage collaboratif. Le constructivisme, auquel certains accordent, à tort, valeur de théorie ou de stratégie d'apprentissage, est un paradigme qui propose une conception de la connaissance faisant de l'apprentissage un acte de collaboration. Le cognitivisme, par ses théories psychocognitives et sociocognitives, est à la base de la dualité qui caractérise l'apprentissage collaboratif. Il propose de tenir compte des mécanismes mentaux et $\mathrm{du}$ fonctionnement cognitif des individus autant que des aspects sociaux de l'apprentissage. Il met également 
en lumière la complémentarité et l'interdépendance de l'apprentissage individuel et collectif. La théorie de la flexibilité cognitive s'intéresse à l'épistémologie de la connaissance. Elle nous amène à comprendre que la démarche collaborative, en misant sur la présentation d'une multiplicité de points de vue, convient à l'apprentissage de contenus complexes. La notion de cognition répartie se révèle aussi utile à la conception des démarches et des environnements de collaboration en proposant d'exploiter la diversité des cognitions présentes dans le groupe et dans l'environnement. Les principes andragogiques sont évoqués pour montrer leur compatibilité avec la démarche d'apprentissage collaboratif. Ils viennent soutenir les assises cognitives et sociales de l'apprentissage et souligner l'importance du respect de l'autonomie de l'apprenant et des efforts pour en favoriser la croissance.

En terminant notre étude des fondements de l'apprentissage collaboratif, nous comparons les démarches de coopération et de collaboration afin de relever ce qui les distingue. Cette comparaison renforce l'idée selon laquelle l'apprentissage collaboratif est adapté aux apprenants qui savent faire preuve de maturité et d'autonomie dans leur apprentissage. Nous concluons ce chapitre en formulant une définition qui, tout au long de l'ouvrage, permettra d'appréhender la démarche d'apprentissage collaboratif et d'en préciser le fonctionnement.

\section{L'APPRENTISSAGE SELON LES CONSTRUCTIVISTES}

En s'opposant au réalisme, le constructivisme adopte une vision subjectiviste du monde qu'on associe aux positions épistémologiques de Piaget, Von Foerster, Bateson et Varela (Wilson, 1992). L'essence du paradigme constructiviste s'exprime par les conceptions qu'il propose de la réalité, de la connaissance, de l'interaction humaine et de la science. Les positions de ce courant de pensée peuvent se résumer ainsi :

- La nature de la réalité. Les représentations mentales sont des réalités ontologiques; elles existent au même titre que l'individu et que tout ce qui se trouve à l'extérieur de lui. 
- La nature de la connaissance. Les connaissances sont construites par l'individu dans son univers mental; ce ne sont pas des réalités qui existent à l'extérieur de l'individu.

- La nature de l'interaction humaine. L'interaction humaine est un processus qui aboutit à un entendement commun, à une compréhension partagée du monde. C'est en échangeant, en partageant, en discutant et en confrontant nos idées à celles des autres qu'on arrive à comprendre le monde et à lui donner un sens. Ces échanges sont faits de coopération et de négociation sociale, plutôt que d'acceptation d'une vision imposée d'autorité.

- La nature de la science. La science est une activité qui cherche le sens à donner au monde; comme toutes les activités humaines, elle possède un caractère subjectif.

\section{LES CONNAISSANCES : DES ENTITÉS SUBJECTIVES EN QUÊTE DE VIABILITÉ}

Pour les constructivistes, les connaissances sont des fabrications individuelles qui résultent de l'expérience personnelle. Ainsi, les constructions mentales se rapportant à une même réalité extérieure diffèrent d'un individu à l'autre. Or, comme les connaissances ne sont pas les mêmes pour tous, on ne peut les considérer comme des entités uniformes et universelles. En fait, les connaissances correspondent à des réalités subjectives et intérieures. Ce que nous reconnaissons être des connaissances ne sont pas des vérités absolues; ce sont simplement des interprétations viables de notre monde élaborées à un moment donné (Resnick, 1987). Elles n'ont de valeur que dans la mesure où elles sont socialement acceptées. Sur ce point, la position constructiviste s'oppose à la tradition scientifique.

La science accorde aux connaissances valeur de vérité ; elles sont censées être conformes à la réalité extérieure et doivent permettre de l'expliquer. Toute vision d'un phénomène qui ne satisfait pas au critère d'explicabilité est qualifiée de croyance, de préconception ou de conception erronée ou naïve. À cette théorie de la vérité, fondée sur la correspondance entre connaissance et réalité, les constructivistes objectent que la connaissance 
est évolutive et que sa viabilité dépend du consensus social qu'elle suscite. On s'appuie souvent sur l'histoire des sciences pour montrer que peu de théories ou de propositions scientifiques résistent au temps. En effet, il y a eu des époques où l'on croyait que la Terre était plate, que le Soleil tournait autour de la Terre ou encore que les plus petites particules de la matière étaient des électrons, des neutrons et des protons. Ces connaissances, qui plus tard ont été invalidées ou dépassées, étaient alors viables. À un moment donné de l'histoire, elles ont obtenu l'assentiment général; tous s'entendaient pour dire que les concepts et les principes provenant de ces représentations fournissaient les meilleures interprétations du monde.

\section{POUR UNE PÉDAGOGIE DE COLLABORATION ET DE COOPÉRATION}

Le constructivisme prône une pédagogie de l'interaction humaine reposant sur deux principes fondamentaux.

- Les connaissances sont construites par l'apprenant en fonction de trois principaux éléments : la structure du domaine étudié, l'expérience de l'apprenant et le contexte dans lequel les connaissances seront utilisées.

- Les connaissances font l'objet d'une négociation dans le cadre d'interactions marquées par la collaboration et la coopération entre les différents acteurs de la formation.

Les notions de subjectivité et de viabilité des connaissances sont ici fondamentales; elles conditionnent le processus d'apprentissage qui s'emploie à rechercher un entendement par la négociation et la collaboration. L'apprentissage se déroule dans un contexte social où l'apprenant partage et confronte ses idées et ses points de vue avec ceux de ses pairs et ceux de la société. Cette interaction l'amène à construire ses connaissances et à dégager une compréhension de la réalité. Par la négociation sociale, en groupe, l'apprenant vérifie la compatibilité de ses propres représentations et de ses visions du monde; il prend la mesure de ses connaissances et de leur viabilité. 
En résumé, la pédagogie constructiviste reconnaît la multiplicité des modes de pensée et admet que les connaissances varient d'un individu à l'autre. Elle mise essentiellement sur les interactions entre l'apprenant et son environnement social pour donner un sens aux connaissances qu'il construit (Tomlinson et Henderson, 1995).

\section{POUR UNE PÉDAGOGIE RÉFLEXIVE}

En plus d'exposer les apprenants à des visions multiples du monde, les situations d'apprentissage constructivistes ont un caractère réflexif. Elles invitent l'apprenant à procéder à un examen critique de ses connaissances et de ses expériences (Bertrand, 1990), à faire un retour sur ses propres processus cognitifs et à évaluer leur efficacité. C'est une pédagogie exigeante qui propose à l'apprenant une démarche de retour sur soi pour mettre au jour la compréhension intuitive des phénomènes étudiés. Elle veut l'aider à prendre conscience de ses propres compréhensions, à éprouver des confusions cognitives et à explorer de nouvelles avenues pour comprendre et agir (Schön, 1994). C'est une démarche introspective de découverte du sens personnel à donner aux apprentissages (Jonassen et al., 1995 ; Crotty, 1994).

La pédagogie réflexive veut calquer la pratique professionnelle qui, du point de vue de la science appliquée, est un processus de résolution de problèmes. Le praticien, en cours d'action ou après l'action, s'interroge sur les processus qu'il met en jeu et sur les moyens qu'il utilise pour résoudre le problème qui lui est posé. Il réfléchit sur ses actions, ses résultats, ses manières de faire et ses connaissances intuitives implicites utilisées dans l'action; il se les remémore, les critique, les restructure et les incorpore dans ses actions ultérieures (Schön, 1994).

Les constructivistes proposent à l'apprenant une démarche qui, comme celle du praticien professionnel, est vue comme un processus de construction progressive des connaissances sollicitant fortement les habiletés métacognitives. 


\section{CONTRIBUTION DU CONSTRUCTIVISME À L'APPRENTISSAGE COLLABORATIF}

L'apprentissage collaboratif adhère à la vision subjectiviste de la connaissance et à sa nature profondément sociale. C'est un processus qui privilégie l'expression de divers modes de pensée et de visions multiples du monde en vue de la construction, par l'apprenant, de son propre corpus de connaissances sur le monde. L'apprentissage collaboratif est constructiviste parce qu'il se définit comme un processus mental, interne et individuel, qui s'alimente à un processus de groupe basé sur l'interaction humaine pour assurer l'élaboration de représentations viables. Il se nourrit d'échanges, de mises en commun, de confrontations, de discussions et de «disputes » opposant des points de vue qui provoquent des remises en question et stimulent de nouveaux apprentissages (von Glaserfeld, 1990 et 1995 ; Jonassen et al., 1995).

L'apprentissage collaboratif utilise les interactions de groupe pour créer sa dynamique, mais il ne se limite pas à la mise en commun des idées. Il suppose un retour sur le processus qui a conduit autant le groupe que l'apprenant à élaborer des représentations et des connaissances nouvelles. L'apprentissage collaboratif est donc un processus dont la dualité inhérente comporte une démarche individuelle et une démarche collective de construction des connaissances.

\section{L'APPRENTISSAGE SELON LES PSYCHOCOGNITIVISTES}

Les théories psychocognitivistes, issues des recherches en psychologie cognitive, tentent d'expliquer comment procède l'apprentissage en décrivant les processus mentaux qui gèrent la construction des connaissances (analyse, raisonnement, validation; préconceptions, images mentales, représentations, etc.). Les recherches dans ce domaine portent sur la structure de la mémoire à long terme et à court terme et sur les stratégies cognitives et métacognitives mises en œuvre pour acquérir les connaissances et résoudre des problèmes. L'étude des stratégies implicites utilisées par les experts a révélé que l'apprentissage est un processus 
dynamique et interactif qui assure une intégration constante de divers éléments et composantes et de nouvelles structures significatives pour l'apprenant (Ericsson et Smith, 1991; Holoyak, 1991). Le processus du traitement de l'information, qui donne lieu à l'élaboration de structures cognitives, est à la base des modèles d'apprentissage psychocognitivistes comme celui élaboré par Anderson et ses collègues (1995). Ces derniers expliquent comment l'apprenant perçoit et emmagasine l'information en créant de nouvelles structures mentales pour ensuite les récupérer et les intégrer aux structures existantes avant de les utiliser.

L'apprentissage n'est donc pas complet si les nouvelles structures ne sont pas intégrées dans les structures antérieures. Pour les psychocognitivistes, trois principaux éléments sont en interaction dans le processus d'apprentissage : les connaissances préalables de l'apprenant (dont on reconnaît l'importance sans toujours savoir exactement comment en tenir compte), l'information dont il dispose et les représentations mentales qu'il élabore.

\section{POUR UNE PÉDAgOGIE DE STRUCTURATION}

Les théories psychocognitivistes appliquées à la pédagogie ont surtout contribué à l'élaboration de méthodes didactiques qui prescrivent le cheminement cognitif de l'apprenant et qui déterminent les conditions à réunir pour créer des situations idéales d'apprentissage. Ainsi, la prise en compte des connaissances préalables, le conflit cognitif, l'accès à des formalismes et la résolution de problèmes font partie des moyens et des méthodes d'enseignement qui amènent les apprenants à insérer les nouvelles connaissances dans des structures cognitives bien ancrées et bien intégrées. Le formateur y joue un rôle primordial et irremplaçable (Bertrand, 1990). Aucun autre intervenant, aucun programme, aucun système ne peut agir en substitut. C'est l'apprenant qui apprend à partir de ses structures propres, mais $c^{\prime}$ est le formateur qui met en place l'environnement didactique indispensable à l'élaboration des connaissances.

Ajoutons que les théories psychocognitivistes ont aussi contribué au développement de systèmes informatiques (hypertexte, hypermédia, tutoriel intelligent, système expert). Inspirés par la 
notion de structures cognitives organisantes, les concepteurs ont utilisé des paradigmes de programmation (décomposition modulaire et fonctionnelle) qui reflètent la théorie d'apprentissage psychocognitiviste.

\section{CONTRIBUTION DES PSYCHOCOGNITIVISTES}

\section{À L'APPRENTISSAGE COLLABORATIF}

Comme nous venons de le voir, les théories psychocognitivistes focalisent surtout sur l'intérieur du système cognitif, sur sa structure et sur son fonctionnement. Elles ne s'intéressent pas à la dimension sociale de l'apprentissage qui demeure un épiphénomène de l'apprentissage individuel (Salomon et Perkins, 1998). Elles portent principalement sur le registre cognitif par opposition à d'autres théories cognitivistes qui prennent en considération ceux de l'affectif et du social comme facteurs déterminants de l'apprentissage.

L'apprentissage collaboratif s'appuie sur les propositions psychocognitives pour élaborer des démarches d'apprentissage mieux adaptées au fonctionnement cognitif des apprenants, des démarches qui respectent les étapes de l'apprentissage et qui sont calquées sur le processus de traitement de l'information. Toutefois, dans les démarches collaboratives, on ne réserve pas au formateur le rôle prédominant que les psychocognitivistes veulent lui accorder. La perspective protégeant la relation d'autorité ou de quasi-autorité entre enseignant et apprenant n'est pas retenue. L'apprentissage collaboratif se tourne plutôt vers les théories sociocognitives pour définir la place et le rôle du formateur.

\section{L'APPRENTISSAGE SELON LES SOCIOCOGNITIVISTES}

Contrairement aux psychocognitivistes qui étudient avant tout le fonctionnement $\mathrm{du}$ mental, les sociocognitivistes s'intéressent surtout au contexte social qui encadre l'apprentissage et qui le nourrit. Inspirés par l'anthropologie et la psychologie sociale, et sans renier les explications de leurs collègues psychocognitivistes sur le fonctionnement cognitif de l'apprentissage, ils estiment que l'apprentissage est soumis à trois variables dominantes: 
1) l'interdépendance entre l'apprenant, l'environnement d'apprentissage et le milieu culturel, 2) l'ancrage social et 3) le transfert des connaissances. Pour percer le processus d'apprentissage, les sociocognitivistes se basent sur des observations anthropologiques décrivant comment l'apprenti est amené, progressivement, à intégrer des gestes, des comportements et des activités qui ressemblent de plus en plus à celles qu'exécute le maître (Lave et Wenger, 1991). Ils décrivent comment se fait l'apprentissage en précisant les caractéristiques de l'environnement d'apprentissage, les éléments d'un contexte idéal d'apprentissage et le rôle que doit jouer le formateur.

Les sociocognitivistes ne font pas de l'apprentissage une démarche exclusivement individuelle et intérieure comme c'est le cas des psychocognitivistes. Ils y ajoutent une dimension sociale qui rend l'apprentissage tributaire de facteurs extérieurs à la personne. Le processus est donc autant individuel que collectif et met en cause deux types de négociations: l'une avec soimême et l'autre avec les personnes du milieu (Harri-Augstein et Thomas, 1991). La négociation individuelle prend la forme d'une interaction entre les structures cognitives élaborées par l'apprenant et la réflexion critique, métacognitive, qu'il mène sur ses propres connaissances. La négociation à caractère social permet à l'apprenant de revoir et de restructurer ses représentations individuelles pour les adapter à la culture du milieu. Dans ce double processus, individuel et collectif, qui ne peut se réaliser que dans le cadre d'interactions de groupe, il importe que les apprenants partagent un même langage, un même univers mental et culturel et un même but (Lave, 1993); autrement, la dynamique entre apprenants devient impossible et l'apprentissage authentique ne peut se réaliser.

\section{POUR UNE PÉDAGOGIE}

\section{MARQUÉE PAR L'ANCRAGE SOCIAL ET CULTUREL}

Selon la pédagogie inspirée des théories sociocognitivistes, pour qu'il y ait apprentissage et que l'apprenant y trouve un sens, la situation pédagogique doit être réelle (Cognition and Technology Group at Vanderbilt, 1990 et 1991 ; Wilson et Cole, 1991). Elle doit de plus permettre aux apprenants de travailler en groupe et 
d'apprendre à apprendre ensemble. Dans ce processus, l'apprenant joue un rôle actif et se sent responsable autant de son apprentissage que de celui des autres. Le rôle du formateur est davantage celui d'un animateur ou d'un guide. Au fur et à mesure que les apprenants développent la capacité d'apprendre de manière autonome et en groupe, le rôle du formateur s'estompe pour laisser graduellement les apprenants prendre en charge leur apprentissage (Garrison, 1995 ; Fisher, 1995 ; Brown et Palincsar, 1987; Collins, Brown et Newman, 1989; Cranton, 1994).

Cette approche soulève la question de la relation entre la dimension individuelle de l'apprentissage et la dimension collective. Qui de l'individu ou du groupe doit avoir la primauté ? Les sociocognitivistes s'entendent pour accorder la primauté à la coordination des actions du groupe dans la construction des connaissances puisque, selon Vygotsky (rapporté par Bertrand, 1990, p. 145), c'est l'interaction sociale qui permet à l'individu de progresser.

\section{CONTRIBUTION DES SOCIOCOGNITIVISTES À L'APPRENTISSAGE COLLABORATIF}

En accord avec l'approche sociocognitive, les démarches de collaboration placent les apprenants dans des situations réelles pour leur permettre de construire, ensemble, leurs connaissances selon le modèle du maître et de l'apprenti et d'en faire le transfert. Le rapport de l'apprenant avec le maître n'est pas celui du modèle traditionnel de l'école, car on ne se situe pas ici dans une perspective de transmission de connaissances. La démarche d'appropriation est dynamique, encadrée par le formateur qui anime, aide et guide les apprenants. Il adapte ses interventions selon le degré d'autonomie du groupe et son objectif ultime est de remettre le plus possible aux apprenants le contrôle de leur apprentissage.

Le processus collaboratif ne dévalue pas le caractère individuel de l'apprentissage au profit du fonctionnement du groupe. Il reconnaît les dimensions individuelle et collective de l'apprentissage et valorise l'interaction entre les deux. Sans négliger de 
fournir un soutien aux apprentissages individuels, le formateur s'intéressera surtout à la coordination des actions du groupe et aux processus collectifs.

\section{LA THÉORIE DE LA FLEXIBILITÉ COGNITIVE}

Dans notre recherche d'une meilleure compréhension de l'apprentissage collaboratif, la théorie de la flexibilité cognitive aide à répondre aux questions suivantes: Quand est-il opportun d'appliquer la démarche collaborative? Est-ce une méthode universelle applicable à l'acquisition de tous les types de connaissances? Est-il approprié de proposer des démarches collaboratives pour l'atteinte de tous les objectifs d'apprentissage?

En s'intéressant à l'épistémologie de la connaissance, la théorie de la flexibilité cognitive nous amène à comprendre que les démarches collaboratives servent mieux que d'autres les apprentissages reliés à des domaines complexes, peu ou mal structurés. Car la collaboration, qui est faite de la mise en commun de visions et de points de vue, permet de fournir des explications diverses d'un même phénomène et ainsi rendre compte de sa complexité.

\section{LES DOMAINES COMPLEXES, PEU OU MAL STRUCTURÉS}

Pour Spiro et al. (1991), un domaine de connaissances, peu ou mal structuré, possède au moins les deux propriétés suivantes:

- de manière typique, chaque cas d'application des connaissances implique le recours simultané à de multiples structures conceptuelles génériques et complexes (points de vue, principes organisateurs, représentations schématiques, algorithmes, etc.) qui interagissent les unes sur les autres.

- le patron des structures conceptuelles utilisées, leurs interactions et l'effet même de ces interactions varient considérablement d'un cas à l'autre, même s'ils sont tous du même type. 
La médecine et l'histoire, par exemple, sont réputées être des domaines peu structurés. D'un cas à l'autre, d'une situation à l'autre, les facteurs et les combinaisons de facteurs à l'œuvre sont toujours différents. Ces domaines font une large place à l'interprétation, à la formulation d'hypothèses et aux spéculations. Par ailleurs, on peut dire avec Spiro que les domaines de connaissances qualifiés de mieux structurés, tels que les mathématiques, ont eux aussi des aspects peu structurés, particulièrement lorsqu'il s'agit de connaissances avancées ou hautement spécialisées. Plus encore, on peut affirmer que tous les domaines dont les connaissances s'appliquent à des situations ou des cas qui ne répondent pas à un ensemble stable de contraintes ou de critères sont, en substance, peu structurés. Par exemple, des règles de base en arithmétique peuvent former un ensemble de connaissances bien structurées, mais lorsqu'il s'agit d'appliquer ces règles pour résoudre un problème issu d'une situation réelle et décrite en langage naturel, il faut faire intervenir un ensemble de connaissances complexes qui se rapportent au processus d'application de ces règles. Cet ensemble de connaissances complexes, appelées métaconnaissances, pourtant inhérent à un domaine bien structuré, est dit peu structuré.

\section{CONNAISSANCES INITIALES ET CONNAISSANCES AVANCÉES}

$\mathrm{Si}$, dans un domaine de connaissances donné, on distingue les connaissances structurées de celles qui le sont moins, on peut aussi appliquer cette distinction aux objectifs d'apprentissage, les uns visant l'acquisition de connaissances initiales (niveau introduction) et les autres se rapportant aux connaissances avancées (niveau spécialisation). Lorsque l'apprenant s'initie à un domaine, ses premiers apprentissages, qui se rapportent le plus souvent à des concepts clés ou à des faits, concernent des connaissances qui peuvent être qualifiées de générales ou «superficielles ». On utilise souvent dans ce cas des stratégies de présentation et d'exposé pour mettre l'apprenant en contact avec le domaine (Smith et Ragan, 1992 ; Yelon, 1996). Les connaissances qui en découlent lui permettent de reproduire grossièrement ce qui a été enseigné. On ne s'attend pas alors à ce qu'il maîtrise toute la complexité du domaine ou qu'il soit en mesure de transférer ses connaissan- 
ces de bases pour étudier et comprendre des cas complexes. Par ailleurs, lorsque l'apprentissage porte sur des connaissances avancées et qu'il propose l'étude de notions exigeant un traitement spécialisé du sujet, le pédagogue se demande quelles situations d'apprentissage sont les mieux adaptées et les plus efficaces pour amener l'apprenant à saisir la complexité et la diversité transversale d'un domaine peu structuré (multiplicité et nonconformité des caractéristiques d'une situation à l'autre).

Toujours selon Spiro, les stratégies qui se sont révélées les plus rentables pour l'apprentissage de connaissances complexes ne sont pas à celles qui sont utilisées avec succès lors des phases d'introduction ou d'initiation à un domaine (segmentation de la matière par bloc, principes généraux, représentation unique et unifiante, etc.). L'application de procédés contraires se montre plus efficace. L'apprentissage de contenus complexes semble plus facile lorsque les apprenants sont exposés, d'une part, à des représentations multiples couvrant tout le domaine et, d'autre part, à une présentation englobante et non segmentée du domaine. Cette approche est exigeante pour l'apprenant et elle lui impose une lourde charge cognitive. Afin d'aider l'apprentissage des connaissances complexes issues de domaines peu structurés, Spiro et ses collaborateurs ont élaboré la théorie de la flexibilité cognitive.

\section{FLEXIBILITÉ COGNITIVE ET APPRENTISSAGE DE CONTENUS COMPLEXES, PEU OU MAL STRUCTURÉS}

Selon l'approche psychocognitiviste, le processus d'apprentissage s'amorce d'abord par la récupération de structures mentales (blocs de connaissances) telles qu'elles sont constituées dans la mémoire. L'apprenant s'appuie sur ces structures pour s'approprier les informations nouvelles et pour construire une nouvelle structure. La théorie de la flexibilité cognitive soutient, pour sa part, que l'utilisation de ces blocs intacts récupérés par l'apprenant n'est pas une opération mentale appropriée à l'apprentissage de contenus complexes. L'apprenant doit d'abord puiser et récupérer des connaissances pertinentes parmi les diverses structures et représentations mentales qu'il possède pour en faire un nouvel assemblage (Li et Jonassen, 1996). C'est à l'aide de cette 
structure initiale nouvellement composée qu'il pourra s'approprier les informations complexes et construire des connaissances avancées. Le processus d'apprentissage est donc celui d'une double construction: la première construction faite à partir des connaissances déjà acquises et des expériences est utilisée pour élaborer les connaissances nouvelles, c'est-à-dire la deuxième construction.

Pour que l'apprenant arrive à maîtriser des connaissances complexes et qu'il puisse en faire le transfert, il doit développer cette souplesse cognitive qui lui permet d'extraire de ses nombreuses structures mentales uniquement les parties pertinentes à la situation d'apprentissage. Il est possible de stimuler la flexibilité cognitive en présentant un même contenu à différents moments de l'apprentissage, selon des perspectives conceptuelles ou des points de vue divers, dans des contextes toujours réorganisés pour l'atteinte d'objectifs différents. Cette méthode, essentielle pour l'acquisition des connaissances complexes, évite la simplification et garantit que l'étude d'un sujet complexe ne se limite pas à une seule vision des choses.

\section{CONTRIBUTION DE LA FLEXIBILITÉ COGNITIVE À L'APPRENTISSAGE COLLABORATIF}

L'apprentissage collaboratif fait sien le principe directeur de la théorie de la flexibilité cognitive, à savoir la nécessité d'offrir plusieurs représentations d'un même objet et de faire place à l'expression de divers modes de pensée pour favoriser l'acquisition de connaissances complexes et en faciliter le transfert. Dans un contexte d'apprentissage collaboratif, le moyen par excellence pour réaliser cette diversité, c'est le groupe, lieu privilégié d'expression de représentations multiples et d'approches diverses. Toutefois, les concepteurs de situations collaboratives doivent être capables de proposer au groupe une problématique ou un objet d'étude d'un niveau de complexité approprié afin de ne pas imposer une charge cognitive trop lourde et risquer la perte de motivation. Ils doivent aussi pouvoir fournir l'aide, les ressources et les outils requis pour que les groupes puissent aborder graduellement l'étude de domaines de plus en plus complexes. 
L'expérience nous montre qu'il ne suffit pas de rassembler des apprenants pour que s'installe la dynamique de collaboration. Parmi les conditions qui doivent être réunies pour susciter l'apprentissage, il faut, entre autres, que la complexité de la problématique proposée soit établie en fonction du groupe. Il nous a été donné d'observer à plusieurs occasions l'échec de forums électroniques dont le but était de susciter la collaboration pour apprendre. La plupart du temps, le manque d'intérêt, la confusion, la banalité, le vide, le silence, l'épuisement rapide du sujet étaient dus au degré de complexité trop faible ou trop élevé des sujets de discussion. La théorie de réflexivité cognitive fournit des pistes précieuses pour nous aider à mieux choisir et définir l'objet d'étude et à établir où se situe le degré de complexité souhaitable pour un groupe donné.

\section{LA COGNITION RÉPARTIE}

Les valeurs associées au constructivisme et les principes d'apprentissage issus du courant cognitiviste posent les assises de l'apprentissage collaboratif, à savoir la reconnaissance de la multiplicité des représentations et des connaissances, qui trouvent leur viabilité par l'ancrage social et culturel; l'interaction entre les pairs et avec le formateur; la négociation et la validation des connaissances; la réflexivité cognitive et sociale; la reconnaissance de la dimension individuelle du processus d'apprentissage; et la primauté à accorder à la coordination des processus collectifs. C'est ce dernier aspect de l'apprentissage collaboratif que le concept de cognition répartie permet de développer en fournissant une meilleure compréhension de la gestion et de la coordination à faire entre les divers types de ressources, humaines et autres, au sein de l'environnement d'apprentissage (Hatano et Inagaki, 1991 ; Pea, 1993 ; Salomon, 1993).

Selon le sens commun, l'intelligence et la connaissance sont des attributs exclusifs de l'homme; elles lui permettent de guider ses actions et de choisir ses comportements. Pourtant, il existe d'autres formes d'intelligence manifestes dans nos environnements; elles existent à l'extérieur de nous. On parle alors de cognitions sociales qui se traduisent dans les buts qu'une société 
se donne, dans les outils qu'on utilise, dans les situations qu'on crée et qu'on partage avec l'entourage. Ces cognitions sociales imprègnent les artefacts, les outils, les habitudes, les traditions, les modes de pensée, les idéologies et les valeurs d'un société.

Les objets, créés par l'homme, sont des «instanciations » de l'intelligence collective d'une société (Pea, 1993; Hastie et Pennington, 1993). Nous les utilisons régulièrement sans être pleinement conscients qu'ils augmentent notre propre cognition, qu'ils y participent et qu'ils l'influencent. Ainsi, lorsque l'individu s'adonne à une activité, il utilise ses ressources de cognition, mais aussi l'intelligence tacite qui existe autour de lui, celle qui est présente dans son environnement. Lave (1993) a repensé l'apprentissage en fonction de l'héritage social, culturel et historique et elle suggère que l'apprentissage n'est pas seulement le fruit de l'interaction sociale ou de l'intelligence individuelle, mais plutôt le résultat de la synergie des deux. Elle conçoit l'apprentissage comme une activité qui fait participer l'apprenant à un monde réel. Cette activité lui permet de s'approprier graduellement les connaissances du «maître» ou de l'expert en utilisant sa capacité de cognition et en faisant intervenir l'intelligence collective, ou cognitions sociales, de son environnement ou de son entourage. Ce faisant, l'apprenant développe également son appartenance à une collectivité.

La cognition n'est donc pas une entité que l'on possède, elle s'accomplit et se met en œuvre par l'exploitation des ressources qui structurent l'activité et qui la rendent possible. Ces ressources sont des personnes, l'environnement, des événements, des situations mais aussi des outils ou des machines (Pea, 1993). Dans l'apprentissage, la cognition en cause n'est donc pas uniquement celle de l'apprenant. Elle est partagée entre au moins trois entités: les acteurs qui participent au processus, les composantes de l'environnement et la situation elle-même. Dans les situations d'apprentissage, à part celle que l'on associe aux personnes, on retrouve assez fréquemment trois autres formes de cognitions: la participation guidée, les langages et les outils informatiques (Pea, 1993). La participation guidée est une cognition qui résulte de l'interaction entre le formateur et les apprenants. Les langages (symbolique, sémantique, mathématique, etc.) ont le pouvoir de 
traiter différentes situations ; pour l'apprenant, il s'agit de choisir le langage qui convient le mieux à la situation et d'en faire une utilisation créatrice au bon moment. Les outils informatiques ont la capacité de réorganiser le fonctionnement mental ; en les exploitant, l'apprenant peut envisager d'autres modes de fonctionnement et ne pas se limiter uniquement à améliorer ceux qu'il connaît déjà. Le cas qui suit, donné en exemple, illustre ces trois formes de cognition. Un formateur s'entend avec un apprenant (participation guidée pour soutenir l'apprentissage) pour qu'il fasse un diagramme avant de construire une équation (choix des bons langages pour traiter adéquatement le problème). L'apprenant utilise alors un logiciel qui permet de créer des représentations (outil informatique pour explorer un nouveau fonctionnement mental).

Plusieurs auteurs reconnaissent l'importance de faire place au concept de cognition répartie pour concevoir des situations et des environnements d'apprentissage. Il s'agit alors de prévoir la synergie des cognitions inhérentes aux apprenants, au groupe d'apprenants, au formateur, aux outils et à tous les autres éléments de l'environnement. C'est au groupe que Pea (1993) attribue les plus puissantes ressources de cognition. Le groupe est comme une vaste mémoire dont la capacité cognitive est grandement supérieure à celle d'un individu. Il peut mettre en œuvre un éventail beaucoup plus large de stratégies de repérage et de traitement de l'information. Cependant, chaque groupe possède un répertoire fini de stratégies qui varie selon le domaine et le profil du groupe: sa taille, sa culture, la dynamique des interactions entre les personnes, la distribution du savoir entre les membres et le mode d'organisation pratique du travail à effectuer. La cognition dans le contexte d'un groupe d'apprentissage se partage et se traduit dans la formulation du but commun, dans les ressources et les outils disponibles, dans les habiletés et les compétences des participants et dans l'information disponible sur le sujet à traiter. 


\section{CONTRIBUTION DE LA COGNITION RÉPARTIE À L'APPRENTISSAGE COLLABORATIF}

Du point de vue pédagogique, l'association du concept de cognition aux personnes, mais aussi à des situations et à des objets, ouvre une nouvelle perspective pour la conception d'activités d'apprentissage et le développement d'environnements informatisés de collaboration. Elle pose comme défi d'exploiter les cognitions des apprenants, individuelles et de groupe, par l'intermédiaire de technologies qui, à leur tour, deviennent des sources de cognitions. Ces environnements d'apprentissage virtuels qui se veulent plus riches exigent, en revanche, que l'apprenant puisse mettre en œuvre des habiletés et des compétences qui sont différentes de celles requises dans un environnement présentiel et traditionnel. Le groupe, le but qu'il se fixe et les outils de communication et de collaboration qu'il doit alors utiliser servent à développer une nouvelle culture induisant une nouvelle façon de faire, des normes et une dynamique sociale particulière.

La conception d'une démarche de collaboration ne se limite donc pas au scénario des interactions entre apprenants. Elle s'intéresse à la composition de l'environnement d'apprentissage et à l'exploitation optimale des autres formes de cognition qui y sont présentes - outils de communication et de travail, sources diverses d'information, intervenants, etc. La démarche collaborative doit proposer à l'apprenant des actions à la mesure de sa cognition, mais elle doit aussi orchestrer l'apport des autres sources de cognition pour l'amener à élargir sa propre cognition. Si nous réussissions à concevoir de telles démarches et de tels environnements, l'apprenant devrait graduellement être amené à faire des apprentissages de haut niveau et de plus en plus complexes.

\section{LES PRINCIPES ANDRAGOGIQUES}

L'andragogie s'inspire du courant humaniste et s'intéresse à l'apprentissage des adultes. En adoptant une démarche empirique, l'andragogie étudie les caractéristiques de l'adulte en situation d'apprentissage et détermine les conditions favorables pour apprendre. Malgré son approche fort différente de celles 
des cognitivistes, elle arrive à des propostions similaires. Les valeurs andragogiques s'accordent avec celles du constructivisme et la conception de l'apprentissage préconisée recoupe les préoccupations des théories sociocognitives. En andragogie, toute situation d'apprentissage doit permettre:

- l'établissement d'un climat d'apprentissage égalitaire et convivial ;

- la prise en compte de l'expérience et des acquis antérieurs de l'apprenant (habileté à la prise de décision, à la résolution de problème, autonomie, capacité d'adaptation, motivation intrinsèque, etc.) ;

- le juste équilibre entre, d'une part, la structure imposée par la démarche d'apprentissage et le matériel pédagogique et, d'autre part, le degré d'autonomie laissé à l'apprenant pour faire des choix relatifs au contenu de ses apprentissages et à leur mode de réalisation ;

- le recours à des méthodes pédagogiques qui permettent la discussion.

Ces principes ont été élaborés à partir de très nombreuses études (Bohlin, Milheim et Viechnicki, 1994 ; Verduin et Clark, 1991 ; Brookfield, 1986 ; Beder et Darkenwald, 1982 ; Cross, 1981 ; Knowles, 1990) qui tendent à démontrer que lorsque l'adulte décide d'entreprendre un apprentissage, il est en général motivé et décidé à fournir les efforts nécessaires pour apprendre, et cela, plus que ce qui a pu être observé chez les adolescents. Selon ces études, l'adulte se distingue également des plus jeunes apprenants par son désir de travailler de manière indépendante. Les études montrent aussi que les adultes se sentent à l'aise et réussissent bien dans un environnement qui exige d'eux qu'ils soient responsables de leur apprentissage et qu'ils collaborent.

Selon Knowles (1990), l'apprentissage proposé par le modèle andragogique repose sur six variables: 1) le besoin de l'adulte de savoir pourquoi il apprend, 2) le concept de soi et le sentiment chez l'apprenant d'être pleinement responsable de son apprentissage, 3) le rôle de l'expérience de l'apprenant qui doit être exploité, 4) la volonté d'apprendre à condition d'y trouver un gain réel, 5) l'orientation que l'adulte veut bien donner à son apprentissage et 6) sa motivation à apprendre. 


\section{CONTRIBUTION DE L'ANDRAGOGIE}

\section{À L'APPRENTISSAGE COLLABORATIF}

Sans apporter d'éléments véritablement nouveaux, les principes andragogiques viennent appuyer les assises cognitives et sociales de l'approche collaborative et renforcer l'idée que la démarche de collaboration convient davantage aux apprenants qui ont acquis un certain degré de maturité et qui peuvent faire preuve d'autonomie dans leur cheminement d'apprentissage et en rapport avec le domaine étudié.

Le profil recherché pour l'apprenant collaboratif recoupe celui que propose l'andragogie et qu'elle décrit comme étant celui de l'adulte qui, en raison de son expérience et de sa situation de vie, apprend de manière plus autonome que les plus jeunes et s'attend à ce que ses apprentissages soient immédiatement transférables. Mais, aujourd'hui, nos systèmes d'éducation veulent développer chez les élèves ce profil d'apprenant autonome et responsable. Dans cette optique, la possibilité d'adopter une démarche collaborative ne serait donc pas exclue pour les apprenants plus jeunes. Ce ne serait alors pas le critère de l'âge qui amènerait l'éducateur ou le formateur à proposer une démarche collaborative, mais plutôt ceux de la maturité et de l'autonomie de l'apprenant.

\section{LA DISTINCTION ENTRE L'APPRENTISSAGE COOPÉRATIF ET L'APPRENTISSAGE COLLABORATIF}

Les éléments théoriques que nous venons de présenter composent la toile de fond de l'apprentissage collaboratif sans toutefois en proposer une définition formelle. Quelques auteurs comme Lebow (1991) ont tenté de cerner le phénomène de la collaboration dans l'apprentissage en posant son système axiologique et en décrivant les principales caractéristiques de la démarche de collaboration.

Selon Lebow, l'apprentissage collaboratif est une conception fondée sur sept valeurs puisées dans la philosophie constructiviste: collaboration, autonomie, réflexivité, générativité, engagement actif, pertinence personnelle et pluralisme En s'appuyant 
sur les théories cognitivistes, il propose à l'apprenant de s'engager dans un processus dynamique et réflexif de construction des connaissances qui se caractérise de la manière suivante.

La démarche collaborative offre à l'apprenant :

- d'explorer les connaissances d'un domaine pour en dégager la structure plutôt que l'assimilation d'un contenu dont les composantes et la structure sont imposées ;

- de réaliser ses apprentissages dans des situations réelles, authentiques ;

- de participer de manière active et soutenue aux interactions de groupe ;

- de développer progressivement son autonomie et sa capacité d'interagir de manière efficace ;

- de développer des compétences de haut niveau: analyse, synthèse, résolution de problème et évaluation.

Par ailleurs, elle exige de lui :

- qu'il mette en œuvre des stratégies cognitives et métacognitives efficaces pour exploiter les ressources de cognition disponibles dans l'environnement d'apprentissage ;

- qu'il s'engage envers le groupe et poursuive le but commun ;

- qu'il accepte de fonctionner dans un cadre de soutien mutuel entre apprenants ;

- qu'il participe à la synergie du groupe pour élaborer des connaissances complexes par la discussion et la négociation du sens.

En plus de cette synthèse, nous proposons, pour notre part, de mieux comprendre l'apprentissage collaboratif en le comparant à l'apprentissage coopératif. Ce procédé nous permet de résumer ce qu'est la coopération et de relever les similitudes entre coopération et collaboration tout en faisant ressortir que la démarche de collaboration convient davantage aux apprenants autonomes, ayant acquis une certaine maturité et se sentant responsable de leur apprentissage. 
Nous avons noté, comme Abrami et al. (1995) et Lewis (1996a et 1996b), que, dans les écrits, les expressions démarche collaborative et démarche coopérative sont, le plus souvent, utilisées indifféremment pour désigner le processus en cours au sein d'équipes ou de groupes. Les démarches sont confondues autant lorsqu'on parle d'apprentissage que lorsqu'on rapporte des démarches de travail dans les organisations. Nous tenterons de lever le flou qui existe entre coopération et collaboration en présentant les caractéristiques de ces deux démarches.

\section{MATURITÉ, CONTRÔLE ET AUTONOMIE}

Pour un groupe donné, le choix entre une démarche coopérative ou collaborative se fait selon la maturité des apprenants, leur autonomie et leur capacité de contrôler leur apprentissage. La démarche coopérative est plutôt adaptée au profil des jeunes apprenants ; elle convient à ceux qui sont moins autonomes, qui n'ont pas acquis beaucoup de maturité cognitive et qui ne possèdent pas encore un répertoire élaboré de stratégies d'apprentissage. Structurée et encadrante, la démarche coopérative réserve au formateur ou à l'enseignant un contrôle sur l'apprentissage. Mais ce contrôle s'exerce de manière éclairée dans le but d'aider l'apprenant à développer graduellement des habiletés de collaboration et à acquérir une plus grande autonomie. La démarche de coopération poursuit donc un double but: apprendre le contenu, mais aussi apprendre à travailler en groupe de manière efficace et à devenir autonome.

La démarche collaborative poursuit essentiellement les mêmes buts à la différence qu'elle est plus souple et qu'elle offre aux apprenants plus de liberté; elle convient davantage aux adultes. Mais pour ceux qui n'ont pas encore acquis la maturité nécessaire pour s'engager dans une réelle collaboration, on pourra adapter la démarche de collaboration en proposant un juste dosage entre le contrôle exercé sur l'apprenant par le formateur et l'autonomie qui lui est accordée.

Pour savoir s'il est plus approprié d'utiliser une démarche de coopération, le formateur fait le diagnostic des habiletés des apprenants, évalue leur degré de maturité et leur capacité de travailler en groupe. Il prend des décisions conséquentes quant 
Figure 1.1

DE LA COOPÉRATION À LA COLLABORATION : UN EXERCICE DE CROISSANCE VERS L'AUTONOMIE

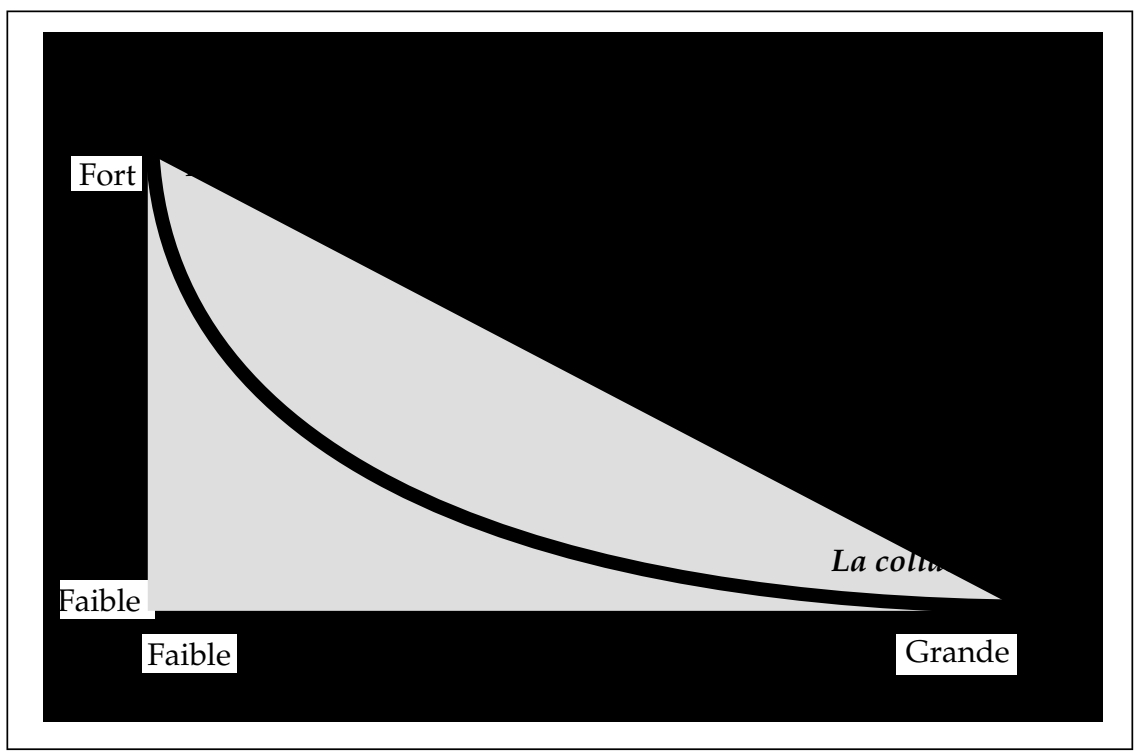

à la liberté qui leur sera laissée: liberté de faire des choix en rapport avec certains aspects du contenu de l'apprentissage et les stratégies à mettre en œuvre pour apprendre.

Des auteurs comme Adams et Hamm (1990) et Cavalier, Klein et Cavalier (1995) conçoivent la démarche coopérative comme une méthode d'initiation ou de préparation à la collaboration véritable. Inspirées par la même philosophie, la coopération et la collaboration se situent sur un continuum. L'apprenant est amené à passer de l'une à l'autre en prenant de plus en plus de décisions et de responsabilités concernant son apprentissage. Ce qui distingue les deux démarches, c'est le degré d'autonomie des apprenants et le contrôle qu'ils exercent sur leur apprentissage (voir figure 1.1).

\section{LE BUT PARTAGÉ}

On s'entend généralement pour dire que les groupes coopératifs et collaboratifs agissent et travaillent pour atteindre un but commun ou partagé. Cole (1993) tient cependant à nuancer cette 
proposition en nous rappelant les deux sens que l'on peut donner au mot "partage". D'une part, le partage renvoie à la division $d^{\prime}$ un tout en plusieurs parties. D'autre part, le partage peut aussi vouloir dire prendre part ou participer à quelque chose. Cole observe que la coopération entend le partage dans le sens de «division » alors que dans le cadre d'une collaboration, il signifie «participer». Nous trouvons cette nuance fort utile pour nous aider à distinguer les deux modes de fonctionnement.

En effet, la coopération, comme l'indiquent Abrami et al. (1995), repose sur la division des tâches et des responsabilités au sein du groupe. Chaque membre est responsable de poser un geste, de mener une action ou d'accomplir une sous-tâche. L'ensemble de ces gestes, de ces actions et de ces activités conduit le groupe au but (voir figure 1.2). C'est le groupe comme entité qui atteint le but; c'est lui qui réalise la tâche de laquelle ressort une production collective. Chaque apprenant participe à l'atteinte de ce but par un apport spécifique à l'œuvre collective.

Dans un contexte de collaboration, les membres du groupe se donnent également un but commun. Mais ce n'est pas uniquement le groupe qui, par ses activités, travaillera à l'atteinte du but : chaque membre, individuellement, cherchera à atteindre par lui-même ce but qui fait consensus au sein du groupe. Il en

Figure 1.2

MODALITÉ DE LA TÂCHE COOPÉRATIVE

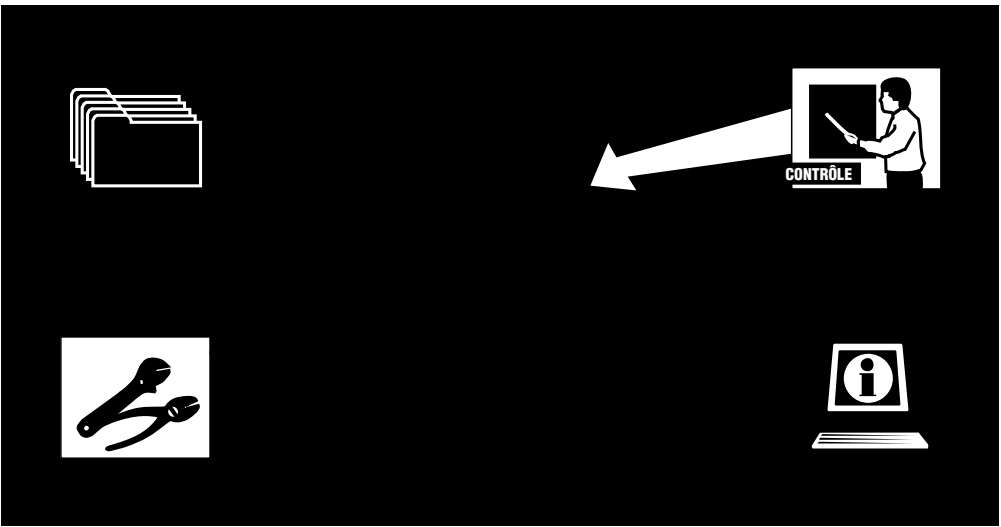


Figure 1.3

MODAlité DE LA TÂCHE COLLABORATIVE

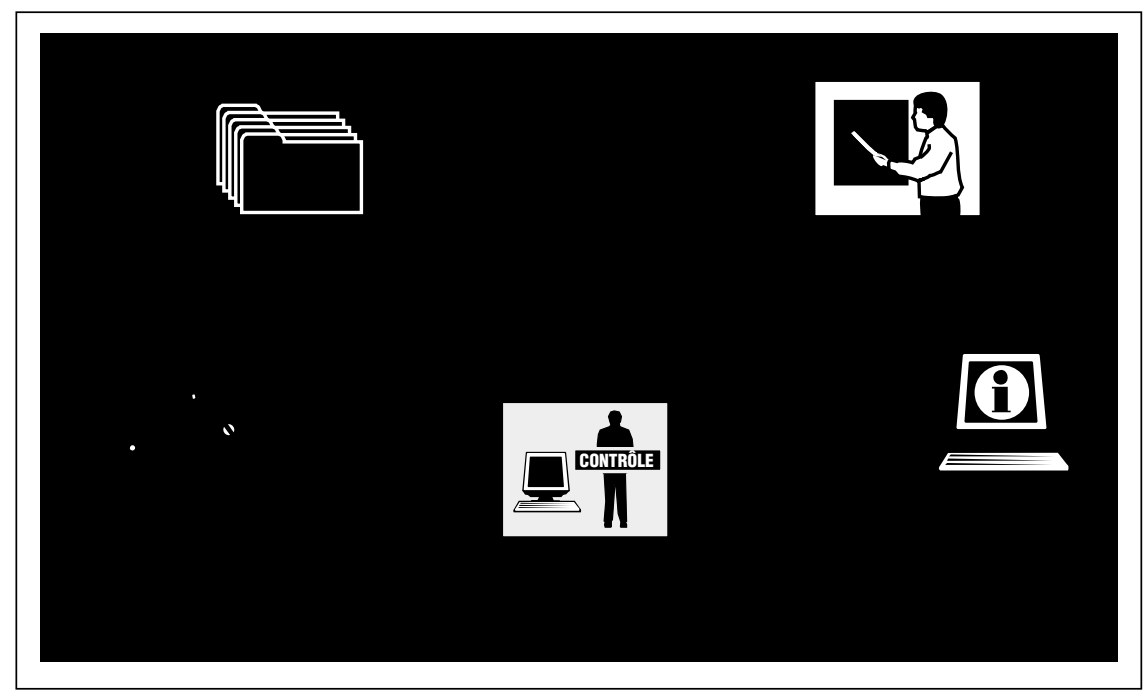

résultera plusieurs productions, une production collective et les productions individuelles des apprenants (voir figure 1.3). L'analyse des tâches collaboratives et coopératives qui suit aide à mieux saisir les différentes façons d'envisager le but en coopération et en collaboration.

\section{LA TÂCHE}

La tâche collaborative se compose d'un ensemble d'activités ou de sous-tâches consignées dans un scénario d'apprentissage qui mène à l'exploration du contenu, à l'élaboration des représentations, à la communication d'idées et à la construction des connaissances. Mais contrairement à la tâche coopérative, elle n'est pas morcelée en vue d'être distribuée entre les participants. L'apprenant réalise, pour lui et par lui-même, l'ensemble de la tâche en puisant dans les ressources de l'environnement et en s'appuyant sur le groupe qui devient, lui aussi, une ressource. Avec quelques variantes, le groupe reprend la réalisation de la tâche en utilisant les contributions de chacun. Cette démarche collective permet au groupe d'atteindre le but tout en constituant 
un soutien à la démarche individuelle de chaque apprenant. La collaboration procède donc de deux démarches : celle de l'apprenant et celle du groupe. Cette dernière devant fournir à chaque apprenant une partie des ressources et de l'aide dont il a besoin pour réaliser seul la tâche afin d'atteindre le but (voir figure 1.4).

Figure 1.4

LA TÂCHE COLLABORATIVE:

LA MÊME POUR TOUS ET RÉALISÉE PAR TOUS

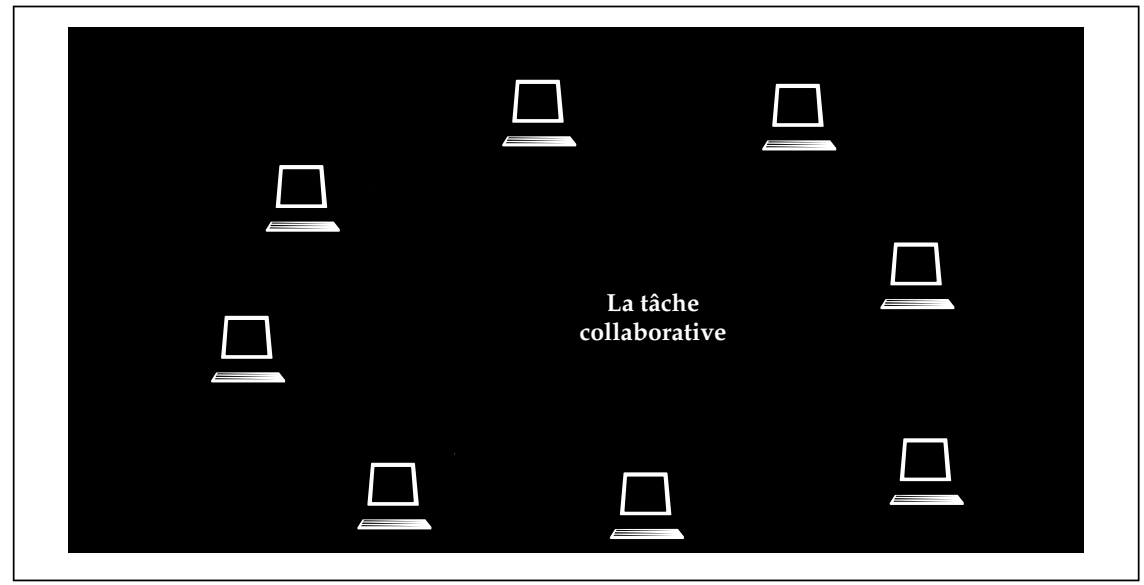

Figure 1.5

LA TÂCHE COOPÉRATIVE:

UN ENSEMBLE DE SOUS-TÂCHES DIFFÉRENTES RÉALISÉES EN ÉQUIPE

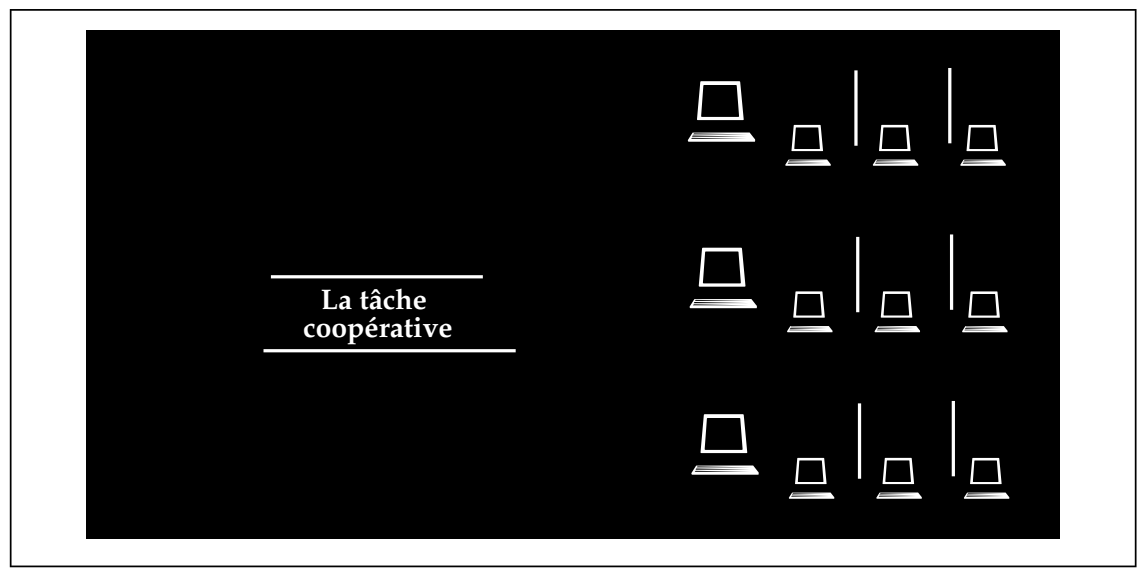


Il en va autrement de la réalisation de la tâche dans la démarche coopérative. L'accomplissement de la tâche se fait par un procédé de spécialisation et adopte une forme pyramidale. Le plus souvent, le groupe est divisé en équipes de deux à cinq apprenants à qui l'on confie des sous-tâches qui peuvent varier d'une équipe à l'autre. Au sein des équipes, chaque membre, individuellement, a une responsabilité spécifique. La tâche est complète lorsque tous les membres de l'équipe ont fait ce qu'ils avaient à faire et que toutes les équipes ont mis en commun leurs réalisations (voir figure 1.5).

\section{L'INTERDÉPENDANCE}

L'interdépendance est un attribut des groupes coopératifs et collaboratifs, mais elle n'a pas la même portée dans les deux cas. Dans la coopération, l'interdépendance entre les apprenants est très forte puisque la contribution des uns n'a de sens que si elle est juxtaposée aux autres ou complétée par le travail des autres. La complémentarité des tâches et leur étroite coordination créent entre les participants une dépendance réciproque. La collaboration, pour sa part, valorise une interdépendance à caractère associatif. Ce qui importe surtout pour les membres, c'est de mettre en commun leurs idées, de faire part de leurs réalisations et de trouver auprès du groupe inspiration, soutien et appui.

Dans les deux cas, l'interdépendance positive doit être encouragée pour que chacun découvre la valeur des autres, leurs compétences et leur expertise afin de pouvoir en bénéficier. Pour stimuler cette interdépendance, les concepteurs de la formation doivent prévoir des activités qui favorisent et facilitent les contacts sociaux, qui permettent le développement du sentiment d'appartenance et stimulent l'engagement envers le groupe. Selon Abrami et al. (1995), l'interdépendance est composée principalement de trois variables: 1) la dynamique sociale, 2) les outils et les moyens de coopération mis à la disposition du groupe et 3) les résultats attendus de l'action du groupe. Par ailleurs, Lundgren-Cayrol (1996) a montré que les techniques utilisées pour constituer les groupes ont une influence considérable sur l'interdépendance; elle se développera de manière fort 
différente entre les membres du groupe si le regroupement se fait au hasard ou selon les affinités de chacun ou encore en fonction des compétences de chacun.

Le tableau 1.1 résume la problématique de l'interdépendance. Dans la première colonne, on retrouve les variables de l'interdépendance propres à la démarche de coopération. La deuxième colonne donne la liste des moyens les plus appropriés pour stimuler ces variables. La troisième colonne rassemble des commentaires sur la pertinence de considérer ces variables dans la démarche collaborative.

\section{EN RÉSUMÉ}

La collaboration se caractérise par des rapports plus égalitaires entre les acteurs : apprenants, formateurs, concepteurs et gestionnaires de formation (Pradl, 1991). Elle préconise un processus plus démocratique que la coopération en donnant aux apprenants plus de pouvoir dans un climat d'ouverture et de responsabilités partagées. En raison de leur maturité, de leur responsabilité et de l'autonomie dont ils jouissent, les apprenants peuvent, tout en participant aux activités du groupe, faire des choix selon leur cheminement et utiliser les stratégies d'apprentissage qui leur conviennent. Pour induire l'apprentissage, la collaboration mise autant sur la réalisation de la tâche par l'apprenant que par le groupe, contrairement à la coopération qui propose à l'apprenant de s'acquitter d'une sous-tâche permettant au groupe de réaliser la tâche. Toutefois, s'il le désire, rien n'empêche un groupe collaboratif de réaliser une tâche selon le mode coopératif. Dans une démarche de collaboration, la tâche coopérative n'est donc pas exclue dans la mesure où elle est l'expression d'un choix fait par le groupe et où elle trouve sa pertinence pour l'atteinte du but.

Le groupe n'est pas l'unique moteur de l'apprentissage collaboratif. Il s'offre comme un moyen d'apprentissage, comme un source de stimulation et de soutien, et sa démarche ne vient pas supplanter celle de l'individu (Boomer, 1990). L'apprenant demeure au centre du processus et son engagement à collaborer repose sur l'intérêt intrinsèque qu'il trouve à partager avec le groupe pour l'aider dans l'accomplissement de la tâche. Le tableau 1.2 résume les caractéristiques des deux démarches. 
Tableau 1.1

INTERDÉPENDANCE EN COOPÉRATION ET EN COLLABORATION

\begin{tabular}{|c|c|c|}
\hline $\begin{array}{l}\text { Variables de l'interdépendance dans la } \\
\text { démarche de coopération }\end{array}$ & $\begin{array}{l}\text { Moyens pour stimuler les variables de } \\
\text { l'interdépendance dans la démarche } \\
\text { coopérative }\end{array}$ & $\begin{array}{l}\text { Pertinence des variables } \\
\text { d'interdépendance pour la démarche } \\
\text { collaborative }\end{array}$ \\
\hline \multicolumn{3}{|l|}{ En rapport avec la dynamique sociale } \\
\hline L'esprit de corps au sein du groupe & $\begin{array}{l}\text { Amener le groupe à se construire une } \\
\text { identité pour stimuler le sentiment } \\
\text { d'appartenance et à réfléchir sur sa } \\
\text { cohésion et sa productivité. }\end{array}$ & $\begin{array}{l}\text { Pertinente. Les activités favorisant la } \\
\text { construction d'une identité sont } \\
\text { importantes à la phase initiale du travail. }\end{array}$ \\
\hline \multicolumn{3}{|l|}{ En rapport avec les outils de coopération } \\
\hline La démarche de réalisation de la tâche & $\begin{array}{l}\text { Décomposer la tâche en sous-tâches; } \\
\text { dresser un plan d'ordonnancement et de } \\
\text { réalisation; préparer un calendrier de } \\
\text { travail. }\end{array}$ & $\begin{array}{l}\text { Moins pertinente. Chaque apprenant doit } \\
\text { pouvoir choisir la stratégie et le } \\
\text { cheminement qui lui conviennent pour } \\
\text { apprendre. Les contributions au travail du } \\
\text { groupe se font selon les habiletés de } \\
\text { chacun. Le groupe s'entend sur la } \\
\text { démarche de collaboration et sur les } \\
\text { modalités du travail. Il trouve lui-même } \\
\text { son équilibre. }\end{array}$ \\
\hline Les ressources communes & $\begin{array}{l}\text { Amener les apprenants à faire connaître } \\
\text { leurs forces et leurs talents et les inviter à } \\
\text { les mettre au service du groupe } \\
\text { (convergence des cognitions). } \\
\text { Identifier et localiser les ressources à } \\
\text { partager pour chaque sous-tâche. }\end{array}$ & $\begin{array}{l}\text { Pertinente. Le partage des ressources est le } \\
\text { moteur de la construction des } \\
\text { connaissances. }\end{array}$ \\
\hline
\end{tabular}


INTERDÉPENDANCE EN COOPÉRATION ET EN COLLABORATION (suite)

\begin{tabular}{|l|l|l|}
\hline $\begin{array}{l}\text { Variables de l'interdépendance dans la } \\
\text { démarche de coopération }\end{array}$ & $\begin{array}{l}\text { Moyens pour stimuler les variables de } \\
\text { l'interdépendance dans la démarche } \\
\text { coopérative }\end{array}$ & $\begin{array}{l}\text { Pertinence des variables } \\
\text { d'interdépendance pour la démarche } \\
\text { collaborative }\end{array}$ \\
\hline La distribution des rôles et des responsabilités & $\begin{array}{l}\text { Attribuer à chaque équipe et à chaque } \\
\text { apprenant un rôle et des responsabilités } \\
\text { distinctes. } \\
\text { Rendre explicite le modèle de } \\
\text { communication au sein du groupe: qui } \\
\text { parle à qui, pour dire quoi, quand, } \\
\text { pourquoi et comment (diade, réseau, } \\
\text { chaîne, cercle, etc.). }\end{array}$ & $\begin{array}{l}\text { Moins pertinente. Chaque apprenant doit } \\
\text { réaliser la tâche pour lui-même. }\end{array}$ \\
L'aménagement de l'environnement & $\begin{array}{l}\text { Prévoir des espaces de travail collectif; } \\
\text { identifier les lieux et les moyens de } \\
\text { communication; faciliter l'accès aux } \\
\text { ressources. }\end{array}$ & $\begin{array}{l}\text { Pertinente. Le groupe établit les règles et } \\
\text { travail contribue à faire prendre conscience } \\
\text { que, sans communication, la collaboration } \\
\text { n'existe pas. }\end{array}$ \\
$\begin{array}{l}\text { Pertinente. L'aménagement de } \\
\text { 'environnement se rapporte au choix et } \\
\text { aux usages qu'on fait des ressources et de } \\
\text { la cognition distribuée. }\end{array}$ \\
\hline En rapport avec les résultats attendus \\
L'atteinte du but & $\begin{array}{l}\text { Donner une même orientation au travail; } \\
\text { s'assurer de la concordance des buts } \\
\text { individuels avec le but commun. }\end{array}$ & $\begin{array}{l}\text { Moins pertinente. À travers l'atteinte d'un } \\
\text { but commun partagé par le groupe, les } \\
\text { membres travaillent pour atteindre leur but } \\
\text { personnel; conciliation des intérêts } \\
\text { personnels et de ceux du groupe. } \\
\text { La reconnaissance et le mérite }\end{array}$ \\
\hline $\begin{array}{l}\text { Moins pertinente. La collaboration est } \\
\text { issue de la participation volontaire et } \\
\text { spontanée. La reconnaissance intrinsèque } \\
\text { est plus significative et plus valorisée. }\end{array}$ \\
\hline
\end{tabular}


Tableau 1.2

\section{COMPARAISON DES DÉMARCHES COOPÉRATIVE ET COLLABORATIVE}

\begin{tabular}{|l|l|l|}
\hline $\begin{array}{l}\text { Variables pédagogiques et } \\
\text { organisationnelles }\end{array}$ & Apprentissage coopératif & Apprentissage collaboratif \\
\hline But de la formation & $\begin{array}{l}\text { Le but se traduit dans des objectifs de } \\
\text { formation formulés par le formateur qui se } \\
\text { rapportent: } \\
\text { à l'apprentissage d'un contenu défini, } \\
\text { - au développement de l'autonomie, } \\
\text { à la préparation à la collaboration. }\end{array}$ & $\begin{array}{l}\text { Dans le cadre du but et des objectifs } \\
\text { définit son but et ses objectifs personnels; il } \\
\text { donne un sens à sa formation. } \\
\text { Ses objectifs incluent le développement de } \\
\text { ses stratégies d'apprentissage et de ses } \\
\text { habiletés de collaboration. }\end{array}$ \\
\hline Atteinte du but & $\begin{array}{l}\text { Le but est commun; il est le même pour } \\
\text { tous. } \\
\text { Il est atteint collectivement par la } \\
\text { réalisation d'activités structurées et de } \\
\text { tâches prédéterminées. }\end{array}$ & $\begin{array}{l}\text { Le but est partagé par le groupe } \\
\text { d'apprenants. Chacun y souscrit et travaille } \\
\text { pour l'atteindre en conciliant ses propres } \\
\text { intérêts, son but et ses objectifs. }\end{array}$ \\
\hline Contenu de formation, objet à connaître & $\begin{array}{l}\text { Le contenu est structuré et présenté par le } \\
\text { formateur. } \\
\text { Les apprenants se l'approprient en réalisant } \\
\text { une tâche de groupe. }\end{array}$ & $\begin{array}{l}\text { Le contenu est un objet à explorer. } \\
\text { L'apprenant l'explore pour en comprendre } \\
\text { la structure et se l'approprier. Cette } \\
\text { démarche se fait individuellement, mais } \\
\text { aussi en groupe. }\end{array}$ \\
\hline Contrôle et autonomie & $\begin{array}{l}\text { Le formateur contrôle l'apprentissage pour } \\
\text { pallier le manque d'autonomie. }\end{array}$ & $\begin{array}{l}\text { Le degré de contrôle que l'apprenant exerce } \\
\text { sur son apprentissage est fonction de sa } \\
\text { maturité et de son autonomie. Le formateur } \\
\text { encourage le fonctionnement autonome de } \\
\text { l'apprenant et du groupe. }\end{array}$ \\
\hline
\end{tabular}


Tableau 1.2

COMPARAISON DES DÉMARCHES COOPÉRATIVE ET COLLABORATIVE (suite)

\begin{tabular}{|l|l|l|}
\hline $\begin{array}{l}\text { Variables pédagogiques et } \\
\text { organisationnelles }\end{array}$ & Apprentissage coopératif & Apprentissage collaboratif \\
\hline Formule pédagogique & $\begin{array}{l}\text { L'apprentissage se fait en équipe. Le travail } \\
\text { réalisé par chaque équipe contribue à } \\
\text { l'œuvre collective. }\end{array}$ & $\begin{array}{l}\text { L'apprentissage résulte du travail } \\
\text { individuel soutenu par des activités de } \\
\text { groupe ou d'équipe. L'apprenant partage } \\
\text { des ressources avec le groupe et utilise le } \\
\text { travail réalisé en groupe pour apprendre. }\end{array}$ \\
\hline Activité pédagogique & $\begin{array}{l}\text { La structure de l'activité pédagogique est } \\
\text { imposée. L'exploration et la découverte du } \\
\text { contenu sont guidées par le formateur } \\
\text { selon une structure imposée. }\end{array}$ & $\begin{array}{l}\text { La structure de l'activité est souple et } \\
\text { ouverte. Les parcours d'exploration et de } \\
\text { découverte sont libres. }\end{array}$ \\
\hline Tâche et sous-tâches & $\begin{array}{l}\text { La tâche est décomposée en sous-tâches qui } \\
\text { sont distribuées aux équipes et aux } \\
\text { apprenants par le formateur. }\end{array}$ & $\begin{array}{l}\text { L'apprenant réalise la tâche et les sous- } \\
\text { tâches, à sa manière. En groupe, les } \\
\text { apprenants réalisent la même tâche ou une } \\
\text { tâche semblable à celle qu'ils réalisent } \\
\text { individuellement. }\end{array}$ \\
\hline Interdépendance & $\begin{array}{l}\text { L'interdépendance est encouragée. Elle est } \\
\text { présente à toutes les étapes. }\end{array}$ & $\begin{array}{l}\text { L'interdépendance sociale et cognitive est } \\
\text { encouragée par le partage des ressources. }\end{array}$ \\
\hline Composition des équipes & $\begin{array}{l}\text { Le formateur décide de la composition des } \\
\text { équipes selon la situation d'apprentissage } \\
\text { et le profil des apprenants. }\end{array}$ & $\begin{array}{l}\text { Les apprenants décident, en accord avec le } \\
\text { formateur, comment les équipes seront } \\
\text { constituées. }\end{array}$ \\
\hline Organisation du groupe et des équipes & $\begin{array}{l}\text { Elle est formelle, méthodique et } \\
\text { systématique. }\end{array}$ & $\begin{array}{l}\text { Elle est souple et déterminée par les } \\
\text { apprenants. }\end{array}$ \\
\hline Participation & Elle est obligatoire. & Elle est volontaire et spontanée. \\
\hline
\end{tabular}


Tableau 1.2

COMPARAISON DES DÉMARCHES COOPÉRATIVE ET COLLABORATIVE (suite)

\begin{tabular}{|l|l|l|}
\hline $\begin{array}{l}\text { Variables pédagogiques et } \\
\text { organisationnelles }\end{array}$ & Apprentissage coopératif & Apprentissage collaboratif \\
\hline Responsabilité du formateur & $\begin{array}{l}\text { Il organise, supervise, guide et encadre } \\
\text { l'apprentissage. Il facilite l'accès aux } \\
\text { ressources. }\end{array}$ & $\begin{array}{l}\text { Il est un facilitateur, une ressource parmi } \\
\text { plusieurs autres. Il fournit de l'aide sur } \\
\text { demande et anime le groupe quand le } \\
\text { besoin est exprimé. }\end{array}$ \\
\hline Responsabilité de l'apprenant & $\begin{array}{l}\text { L'apprenant est responsable de réaliser la } \\
\text { sous-tâche qui lui a été confiée. Son } \\
\text { exécution est coordonnée avec celle des } \\
\text { autres. }\end{array}$ & $\begin{array}{l}\text { L'apprenant est responsable de son } \\
\text { apprentissage et de l'atteinte du but qu'il } \\
\text { s'est fixé. Mais il est aussi responsable de } \\
\text { l'atteinte du but par le groupe. }\end{array}$ \\
\hline Évaluation & $\begin{array}{l}\text { L'évaluation est sommative. Elle porte sur } \\
\text { la production finale du groupe. Le } \\
\text { formateur accorde une note pour le groupe. } \\
\text { Pour développer les habiletés de } \\
\text { collaboration, le formateur évalue les rôles } \\
\text { joués par les apprenants en faisant un } \\
\text { retour sur la démarche du groupe. }\end{array}$ & $\begin{array}{l}\text { L'évaluation est formative. Elle porte sur } \\
\text { les connaissances acquises. Le formateur en } \\
\text { est responsable. } \\
\text { L'apprenant, aidé par le formateur, est } \\
\text { invité à faire un diagnostic de ses stratégies } \\
\text { métacognitives et de son processus } \\
\text { d'apprentissage. } \\
\text { Les apprenants font une appréciation } \\
\text { collective du fonctionnement du groupe et } \\
\text { des équipes (cohésion et productivité). }\end{array}$ \\
\hline
\end{tabular}




\section{VERS UNE DÉFINITION DE L'APPRENTISSAGE COLLABORATIF}

Pour nous, l'apprentissage collaboratif n'est pas une théorie d'apprentissage, mais une démarche en vue de la construction progressive des connaissances. La définition que nous en proposons se base sur le paradigme constructiviste, sur les théories d'apprentissage issues du courant cognitiviste, sur les principes andragogiques et sur la comparaison que nous avons établie entre les démarches coopérative et collaborative. Comme le veut l'approche constructiviste, cette définition est de nature provisoire. Nous l'utiliserons tout au long de ce travail pour nous permettre de progresser. D'une part, pour explorer les processus sociaux et cognitifs qui sont en jeu dans la collaboration et étudier leur fonctionnement et, d'autre part, pour proposer un modèle d'aménagement des environnements virtuels de collaboration et décrire des usages possibles des technologies de l'information et de la communication.

L'apprentissage collaboratif est une démarche active par laquelle l'apprenant travaille à la construction de ses connaissances. Le formateur y joue le rôle de facilitateur des apprentissages alors que le groupe y participe comme source d'information, comme agent de motivation, comme moyen d'entraide et de soutien mutuel et comme lieu privilégié d'interaction pour la construction collective des connaissances. La démarche collaborative reconnaît le caractère individuel et réflexif de l'apprentissage de même que son ancrage social en le raccrochant aux interactions de groupe. En fait, la démarche collaborative couple deux démarches: celle de l'apprenant et celle du groupe.

L'apprenant s'engage à travailler avec les membres du groupe en vue de la réalisation du but commun tout en conciliant ses intérêts et ses objectifs personnels. Il collabore dans le cadre des interactions de groupe en partageant ses découvertes. Les échanges avec le groupe et la réalisation d'une tâche collective lui permettent de partager ses découvertes, de négocier le sens à donner à son travail et de valider ses connaissances nouvellement construites. Dans sa démarche, il fait preuve d'autonomie et assume la responsabilité de son apprentissage tout en se sentant responsable de l'atteinte du but qu'il partage avec tous. 
Le groupe est un catalyseur de l'apprentissage. En se donnant un but, en s'engageant dans la réalisation collective d'une tâche, le groupe, en tant que groupe, apprend et construit des connaissances. Ce faisant, il permet à chaque apprenant de se confronter aux autres et il nourrit les apprentissages de chacun. Dans la démarche collaborative, les apprenants collaborent aux apprentissages du groupe et, en retour, le groupe collabore à ceux des apprenants. 



\section{Chapitre 2}

\section{Environnements d'apprentissage virtuels, communication et collaboration}

\section{L}

distinction que nous venons de faire entre les apprentissages coopératif et collaboratif montre que la démarche collaborative convient aux apprenants qui savent faire preuve d'autonomie et qui sont prêts à s'engager volontairement dans un processus qui combine le travail individuel et collectif. Nous avons vu aussi que cette démarche mise sur des environnements riches où l'apprenant trouve les ressources et les outils nécessaires à la réalisation des activités individuelles et de groupe.

Dans un contexte de formation à distance, la collaboration entre apprenants est rendue possible grâce à des environnements technologiques accessibles à distance. Les espaces communs de partage de ressources, les espaces de communication de groupe et l'espace privé y sont aménagés pour faciliter la collaboration. C'est à travers ceux-ci que la collaboration se développe. L'apprenant, seul dans un espace privé, s'adonne à un dialogue intérieur pour construire de nouvelles connaissances; en groupe, dans l'espace de communication, il échange avec ses pairs pour poursuivre la 
construction de ses connaissances, pour en débattre et pour les valider. Dans l'espace commun, il trouve tous les outils nécessaires pour réaliser le travail qui mène à l'atteinte du but que s'est donné le groupe.

Ce deuxième chapitre traite des environnements d'apprentissage virtuels qui renvoient à une pédagogie ouverte en accord avec les valeurs du constructivisme. Nous y décrivons les espaces de collaboration qui structurent ces environnements avant d'étudier plus particulièrement celui que l'on réserve à la communication de groupe. Nous montrons comment, dans cet espace, la pédagogie de collaboration exploite le forum électronique et l'interaction écrite comme ressources privilégiées de l'apprentissage. On verra alors que le forum se situe au cœur des environnements d'apprentissage virtuels et qu'il fournit des lieux d'échange et de partage où se joue la partie la plus importante de la collaboration et de l'interaction. Nous verrons également quelles conditions doivent être satisfaites pour qu'une collaboration misant sur l'interaction écrite soit fructueuse. Nous terminons en proposant aux concepteurs d'environnements d'apprentissage virtuels d'aménager les forums électroniques en lieux de télétravail, télégestion, télédiscussion et téléassistance afin d'encadrer le travail collaboratif.

\section{LES ENVIRONNEMENTS D'APPRENTISSAGE VIRTUELS}

La notion d'environnement d'apprentissage n'est pas nouvelle ; elle a été exploitée par le courant de la pédagogie ouverte qui conçoit l'école comme un environnement éclaté. Au cours des années 1970, plusieurs applications de cette pédagogie ont vu le jour. L'école ouverte est alors aménagée non plus en fonction des groupes classe, mais en espaces ayant différentes vocations et au sein desquels on trouve diverses ressources: un coin pour l'histoire et la géographie, un autre pour les mathématiques, un autre encore pour le dessin et le bricolage, etc. L'école devient un lieu décloisonné où les élèves des différentes classes accèdent à ces espaces et à ces ressources selon une grille horaire souple et 
variable pour réaliser des travaux individuels et d'équipe (Morlan, 1973). Les groupes classe ont tout de même un quartier général où ils se rassemblent pour recevoir des explications et des consignes, pour planifier et organiser le travail et pour faire le bilan de leurs apprentissages avec l'enseignant. Ce dernier, qui n'est plus la seule source de connaissances, est plus un facilitateur qui offre un enseignement-soutien et pour qui l'apprentissage résulte d'une découverte guidée.

Pour nous, les environnements d'apprentissage virtuels sont envisagés un peu comme des écoles ouvertes et décloisonnées, mais sans connaître les sérieux problèmes d'aménagement des espaces physiques, de gestion et d'accès aux ressources que l'école ouverte a connus. Grâce aux technologies, il est dorénavant possible de créer plus facilement des environnements ouverts où l'on retrouve tous les espaces désirés foisonnant de ressources et rendus accessibles à tous sans contraintes de temps et d'espace.

Aujourd'hui, les milieux de l'éducation, de la formation et du perfectionnement en milieu de travail se tournent vers les environnements $d$ 'apprentissage virtuels espérant y trouver une réponse prometteuse à la demande pressante de modèles d'apprentissage adaptables à différents profils d'apprenants et assez souples pour s'affranchir des contraintes de temps, d'espace et d'accès aux ressources. Mais avant que les environnements d'apprentissage virtuels puissent s'implanter et se répandre dans ces milieux, il reste un long travail d'appropriation à faire et au moins trois grands défis à relever; les voici:

- prendre un virage pédagogique radical qui nous amène à faire de l'apprentissage notre principale problématique plutôt que de nous intéresser en priorité à l'enseignement ;

- adopter de nouvelles méthodes de design pédagogique adaptées à la conception de genre d'environnements ;

- maîtriser un nouveau savoir-faire technologique pour réaliser des environnements conviviaux, faciles à diffuser, à maintenir et à gérer. 


\section{LA NOTION D'ENVIRONNEMENT D'APPRENTISSAGE VIRTUEL}

Pour plusieurs, le terme «environnement d'apprentissage virtuel » renvoie de plus en plus à des univers dynamiques étroitement associés à une vision constructiviste dont le fonctionnement se caractérise essentiellement par l'interaction humaine. Certains autres les envisagent encore comme des produits statiques construits par l'assemblage d'objets technologiques servant uniquement à transmettre de l'information. Nous optons pour le premier entendement et nous reprenons les termes de Bordeleau (1994) qui décrit les environnements d'apprentissage informatisés en rappelant les choix pédagogiques qu'ils soustendent et leurs composantes technologiques.

Organisation spatio-temporelle réelle ou virtuelle constituée d'un ensemble d'éléments en interaction, mondains ou imaginaires, aussi bien des personnes, des machines, des logiciels, des techniques que des conditions susceptibles d'agir sur les activités humaines [...] En qualifiant l'environnement d'apprentissage, on signifie que, parmi les activités humaines, celles qui consistent à apprendre et à apprendre à apprendre doivent être prédominantes. En ajoutant technologique, on signifie que, parmi les éléments en interaction, il y a présence obligatoire de machines, de logiciels, de techniques et de ressources humaines qui se les sont appropriés. Rappelons qu'il faut entendre par technologie à la fois les objets techniques (matériaux, machines, outils, programmes, logiciels) et les procédés de conception, de production ou d'utilisation desdits objets. Dans le domaine qui nous occupe, il s'agit surtout des technologies du monde de l'informatique et de l'électronique (p. 26).

Nous complétons cette définition en y ajoutant la notion de système proposée par Doré et Basque (1998). Dans la perspective de la théorie des systèmes, l'environnement abrite un ou plusieurs systèmes et sous-systèmes. C'est un ensemble organisé de composantes en interaction dont les actions concourent à l'atteinte d'un but. Ainsi, dans un environnement d'apprentissage réel ou virtuel, le formateur, l'apprenant, le groupe, la classe, les manuels et les autres ressources sont des sous-systèmes en interaction qui sont orientés vers le développement de nouvelles connaissances. Doré et Basque situent en outre ces systèmes 
d'apprentissage dans le paradigme constructiviste parce qu'ils sont fondés sur l'exploration des connaissances, sur l'initiative personnelle et la liberté de choix.

Les environnements sont donc des systèmes conçus pour soutenir les communautés d'apprenants qui veulent collaborer. Ils proposent des lieux où les apprenants interagissent et travaillent en utilisant diverses ressources. En raison du degré de liberté permis dans un environnement d'apprentissage, la démarche d'apprentissage n'est pas dictée d'autorité par un formateur. Elle est plutôt proposée à l'apprenant et au groupe puis elle est discutée et négociée dans le but de faire émerger un consensus au regard de la cible à atteindre et pour que les attentes et les responsabilités des uns et des autres soient rendues explicites et comprises par tous. Le rôle du formateur devient alors celui d'un accompagnateur. En somme, sous la notion d'environnement, on retrouve les principes de la collaboration et de démarche négociée et adaptée au groupe dont le parcours, qui se situe à l'intérieur des balises connues, n'est pas entièrement défini à l'avance par le concepteur.

La conception d'environnements d'apprentissage virtuels s'appuie sur le constructivisme et fait appel aux technologies. C'est une tâche complexe qui pose aux concepteurs deux problèmes principaux (Doré et Basque, 1998). Le premier problème se rapporte au caractère flou des environnements qui doivent pouvoir s'adapter aux contingences de démarches libres et aux besoins spécifiques de chaque groupe. Pour résoudre ce problème, les efforts des concepteurs ne doivent plus être mis sur la définition des stratégies pédagogiques, mais plutôt sur l'anticipation des démarches d'apprentissage, sur les besoins des apprenants et sur l'accès aux outils et aux ressources qui seront utilisées.

Le deuxième problème réside dans l'absence de méthodes de conception pédagogique adéquates et le manque de connaissances pragmatiques pour la création de ces environnements. Rappelons que la conception ou «design» pédagogique se veut une démarche scientifique qui vise à trouver et à appliquer des moyens pour favoriser l'apprentissage. Le résultat se traduit par un ensemble de plan et de devis décrivant des activités d'apprentissage et d'enseignement (Paquette, à paraître). En s'appuyant 
sur de solides fondements théoriques, les méthodes de conception pédagogique proposent des interventions pédagogiques conséquentes aux théories de l'apprentissage sur lesquelles elles se fondent. Or celles qui ont été couramment utilisées depuis de nombreuses années - par exemple celles de Mager, 1962, de Briggs (1981), de Romiszowski (1980), de Gagné (1985) ou de Dick et Carey (1985) - ne peuvent tenir compte des nouvelles théories d'apprentissage issues des avancées des sciences cognitives. Elles ne répondent pas non plus aux exigences de la conception d'environnements d'apprentissage médiatisés et informatisés. Plus précisément, elles ne permettent pas de tirer profit de la nature interactive et du potentiel médiatique des technologies (Trollip et Alessi, 1991) ni de tenir compte de la complexité de la situation $\mathrm{d}$ 'apprentissage qui fait intervenir et interagir plusieurs acteurs et des ressources multiples.

Les méthodes de design pédagogique traditionnelles sont centrées sur l'analyse minutieuse du problème de formation. Elles adoptent des démarches de développement séquentielles, par étape et avec des boucles de rétroaction pour produire, de manière systématique et planifiée, un produit fini de formation. La conception d'environnements d'apprentissage virtuels dynamiques ne peut plus s'accommoder de ces méthodes. De nouvelles pratiques de design pédagogique capables d'opérationnaliser et d'intégrer des éléments théoriques de la systémique et du cognitivisme et d'exploiter pleinement les potentialités des technologies doivent être développées (Paquette, à paraître). Des chercheurs sont actuellement à l'œuvre pour élaborer un cadre théorique, des outils méthodologiques de même que des outils logiciels pour répondre aux besoins des concepteurs d'environnements d'apprentissage.

\section{LA CONSTRUCTION D'ENVIRONNEMENTS D'APPRENTISSAGE}

Les percées des sciences cognitives pour décoder les mécanismes de l'apprentissage, l'évolution des technologies de l'information et de communication (multimédia, hypertexte, hypermédia services télématiques, etc.) et l'Internet en véritable explosion comme moyen de diffusion ont placé le concepteur d'environnements d'apprentissage devant des possibilités inouïes. Au Centre 
de recherche LICEF 1 , des chercheurs ont mis en évidence la multiplicité des décisions à prendre pour concevoir de tels environnements et ils ont voulu répondre aux besoins des concepteurs en développant une méthode et des outils d'ingénierie pédagogique. Ils ont mis au point MISA, Méthode d'ingénierie des systèmes d'apprentissage, qui permet de prendre en considération la complexité des systèmes d'apprentissage (Paquette, à paraître). Résolument constructiviste, MISA utilise la technique de modélisation par objet typé (MOT) pour représenter les systèmes d'apprentissage. Comme le montre le tableau 2.1, cette méthode progresse en six phases:

- définition du projet de formation, principes d'orientation de la formation,

- analyse préliminaire,

- architecture du système d'apprentissage,

- préparation des devis du matériel d'apprentissage,

- production du matériel et des environnements sur la base des devis,

- planification de la diffusion.

À chacune de ces phases, la méthode aborde le problème de conception selon quatre axes:

- l'axe des connaissances et des compétences à acquérir,

- l'axe pédagogique,

- l'axe médiatique,

- l'axe de diffusion.

Bien que structurée en phases, en axes pour la réalisation de tâches et d'activités, MISA ne propose pas pour autant une démarche linéaire ; elle fait appel à un processus de résolution de problème de conception en procédant par une démarche en spirale et par itérations successives (Paquette, à paraître). Le tableau qui suit donne une vue d'ensemble des phases et des axes de MISA.

1. Le Centre de recherche LICEF est rattaché à la Télé-université. Il concentre ses travaux dans le domaine de l'informatique cognitive et des environnements de formation. 
Tableau 2.1

Phases ET AXeS De MISA

\begin{tabular}{|c|c|c|c|c|c|c|}
\hline $\begin{array}{l}\text { Phase } 1 \\
\text { Définition du projet } \\
\text { de formation }\end{array}$ & & $\begin{array}{l}\text { Phase } 2 \\
\text { Analyse } \\
\text { préliminaire }\end{array}$ & $\begin{array}{l}\text { Phase } 3 \\
\text { Architecture }\end{array}$ & $\begin{array}{l}\text { Phase } 4 \\
\text { Devis matériels et } \\
\text { des téléservices }\end{array}$ & $\begin{array}{l}\text { Phase } 5 \\
\text { Réalisation et } \\
\text { validation }\end{array}$ & $\begin{array}{l}\text { Phase } 6 \\
\text { Plan de diffusion }\end{array}$ \\
\hline \multirow{4}{*}{$\begin{array}{l}\text { - Profil } \\
\text { organisationnel } \\
\text { - Objectifs du projet } \\
\text { de formation } \\
\text { - Publics cibles } \\
\text { - Contexte actuel } \\
\text { - Ressources existantes }\end{array}$} & $\begin{array}{l}\text { Modèle de } \\
\text { connaissances }\end{array}$ & $\begin{array}{l}\text { - Décrire le } \\
\text { modèle de } \\
\text { connaissances et } \\
\text { le tableau des } \\
\text { compétences. }\end{array}$ & $\begin{array}{l}\text { - Compléter le } \\
\text { modèle de } \\
\text { connaissances et } \\
\text { le tableau des } \\
\text { compétences. }\end{array}$ & $\begin{array}{l}\text { - Décrire les sous- } \\
\text { modèles associés } \\
\text { aux instruments } \\
\text { et aux objets } \\
\text { matériels. }\end{array}$ & & $\begin{array}{l}\text { - Décrire la } \\
\text { gestion des } \\
\text { connaissances. }\end{array}$ \\
\hline & $\begin{array}{l}\text { Modèle } \\
\text { pédagogique }\end{array}$ & $\begin{array}{l}\text { - Décrire le } \\
\text { réseau des } \\
\text { événements } \\
\text { d'apprentissage. }\end{array}$ & $\begin{array}{l}\text { - Compléter le } \\
\text { réseau des } \\
\text { événements } \\
\text { d'apprentissage. } \\
\text { - Décrire les } \\
\text { scénarios } \\
\text { pédagogiques. }\end{array}$ & $\begin{array}{l}\text { - Définir les } \\
\text { propriétés des } \\
\text { instruments et } \\
\text { objets matériels. }\end{array}$ & & $\begin{array}{l}\text { - Décrire la } \\
\text { gestion des } \\
\text { apprenants et } \\
\text { des facilitateurs. }\end{array}$ \\
\hline & $\begin{array}{l}\text { Modèle } \\
\text { médiatique }\end{array}$ & $\begin{array}{l}\text { - Orienter le } \\
\text { modèle des } \\
\text { matériels. }\end{array}$ & $\begin{array}{l}\text { - Décrire } \\
\text { l'infrastructure } \\
\text { de l'ingénierie. } \\
\text { - Orienter le } \\
\text { modèle } \\
\text { médiatique. }\end{array}$ & $\begin{array}{l}\text { - Décrire les } \\
\text { modèles des } \\
\text { matériels et } \\
\text { les outils et } \\
\text { moyens de } \\
\text { communication. } \\
\text { - Définir les } \\
\text { services et les } \\
\text { milieux de } \\
\text { formation. }\end{array}$ & $\begin{array}{l}\text { - Réaliser le } \\
\text { matériel } \\
\text { pédagogique. } \\
\text { - Faire le } \\
\text { registre des } \\
\text { changements. }\end{array}$ & $\begin{array}{l}\text { - Décrire la } \\
\text { gestion du SA, } \\
\text { des matériels et } \\
\text { des ressources. }\end{array}$ \\
\hline & $\begin{array}{l}\text { Modèle de } \\
\text { diffusion }\end{array}$ & $\begin{array}{l}\text { - Orienter le } \\
\text { modèle de } \\
\text { diffusion. } \\
\text { - Faire l'analyse } \\
\text { préliminaire des } \\
\text { coûts-bénéfices- } \\
\text { impacts. }\end{array}$ & $\begin{array}{l}\text { - Orienter le } \\
\text { modèle de } \\
\text { diffusion. }\end{array}$ & $\begin{array}{l}\text { - Décrire le } \\
\text { modèle de } \\
\text { diffusion, } \\
\text { l'infrastructure } \\
\text { organisationnelle } \\
\text { et technologique. }\end{array}$ & $\begin{array}{l}\text { - Établir le plan } \\
\text { des essais et } \\
\text { des tests. } \\
\text { - Définir le } \\
\text { registre des } \\
\text { changements. }\end{array}$ & $\begin{array}{l}\text { - Décrire la } \\
\text { gestion de la } \\
\text { qualité. }\end{array}$ \\
\hline
\end{tabular}


Cette équipe de chercheurs du LICEF a également développé un modèle d'un campus virtuel (figure 2.1) entièrement dédié au téléapprentissage qui s'organise autour de cinq acteurs:

- l'apprenant,

- le concepteur,

- le formateur,

- le gestionnaire de la formation,

- l'expert de contenu.

Chacun de ces acteurs est engagé dans divers processus:

- un processus d'apprentissage,

- un processus de design,

- un processus de tutorat,

- un processus de gestion,

- un processus d'information.

Les ressources du campus virtuel sont réparties dans cinq espaces (figure 2.2) :

- l'espace d'autogestion permet à l'apprenant de planifier, de gérer et de faire le suivi de son apprentissage ;

- l'espace d'information regroupe des documents de tous types ;

- l'espace de production contient les outils nécessaires pour s'acquitter de la tâche d'apprentissage et produire les travaux attendus ;

- l'espace de collaboration réunit des outils et propose des lieux permettant à l'apprenant d'échanger, de participer à des travaux en groupe ou en équipe ;

- l'espace d'assistance permet d'obtenir de l'aide et des conseils de la part d'une personne-ressource en ligne ou du système informatique.

Cette architecture générique, à la disposition des concepteurs, sert de cadre conceptuel au design d'environnements d'apprentissage virtuels alors que MISA offre une méthode pour développer de tels environnements. 


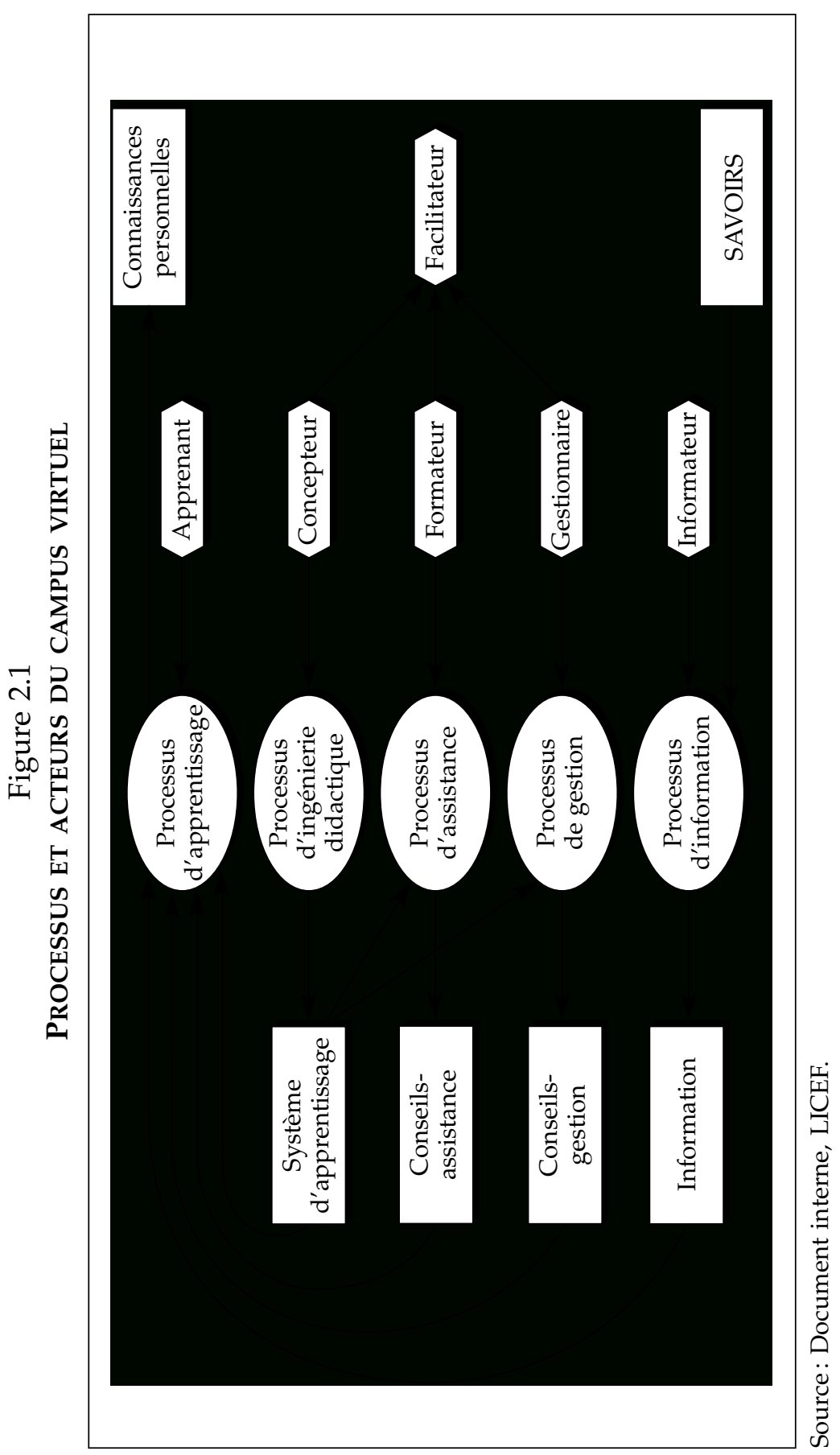


Figure 2.2

CINQ ESPACES DU CAMPUS VIRTUEL

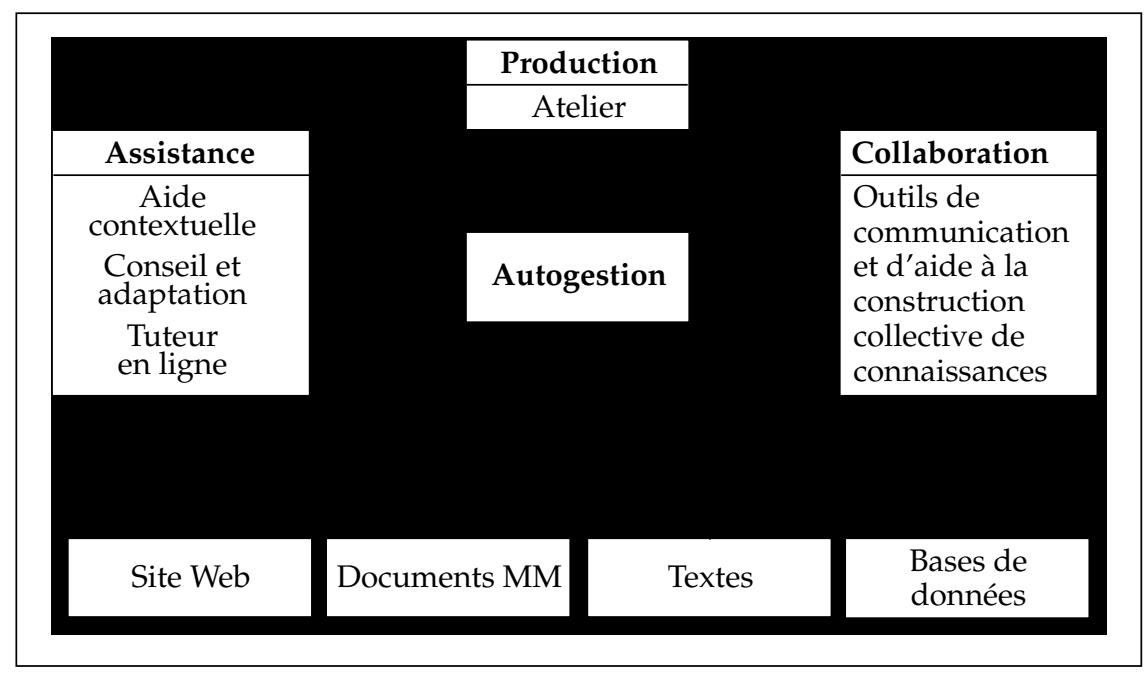

Pour les besoins de la présente étude, nous nous concentrons sur la modélisation de l'espace de collaboration tout en sachant que, dans un environnement d'apprentissage complet, les autres espaces d'un campus virtuel doivent être présents.

\section{L'ESPACE DE COLLABORATION}

La collaboration, quelle que soit sa nature, son but ou son objet, a besoin d'espaces pour exister. Dans ce cas, l'espace peut correspondre à un lieu physique ou virtuel où les collaborateurs se rencontrent pour travailler ensemble. On peut aussi donner à ce terme une valeur métaphorique pour englober des univers d'idées, de sentiments et d'émotions partagés par le groupe. Pris dans un sens concret, l'espace de collaboration peut donc être une salle de réunion où des collaborateurs discutent du projet qui les rassemble, ou un laboratoire où des chercheurs travaillent pour faire avancer un projet commun. Mais ce peut aussi être quelques feuilles de papier sur lesquelles des notes ont été griffonnées au cours d'une conversation informelle ou encore un modèle, un concept ou une hypothèse développés par l'un des collaborateurs et présentés au groupe. En somme, est espace de 
Figure 2.3

ESPACES ET OUTILS DE COLLABORATION

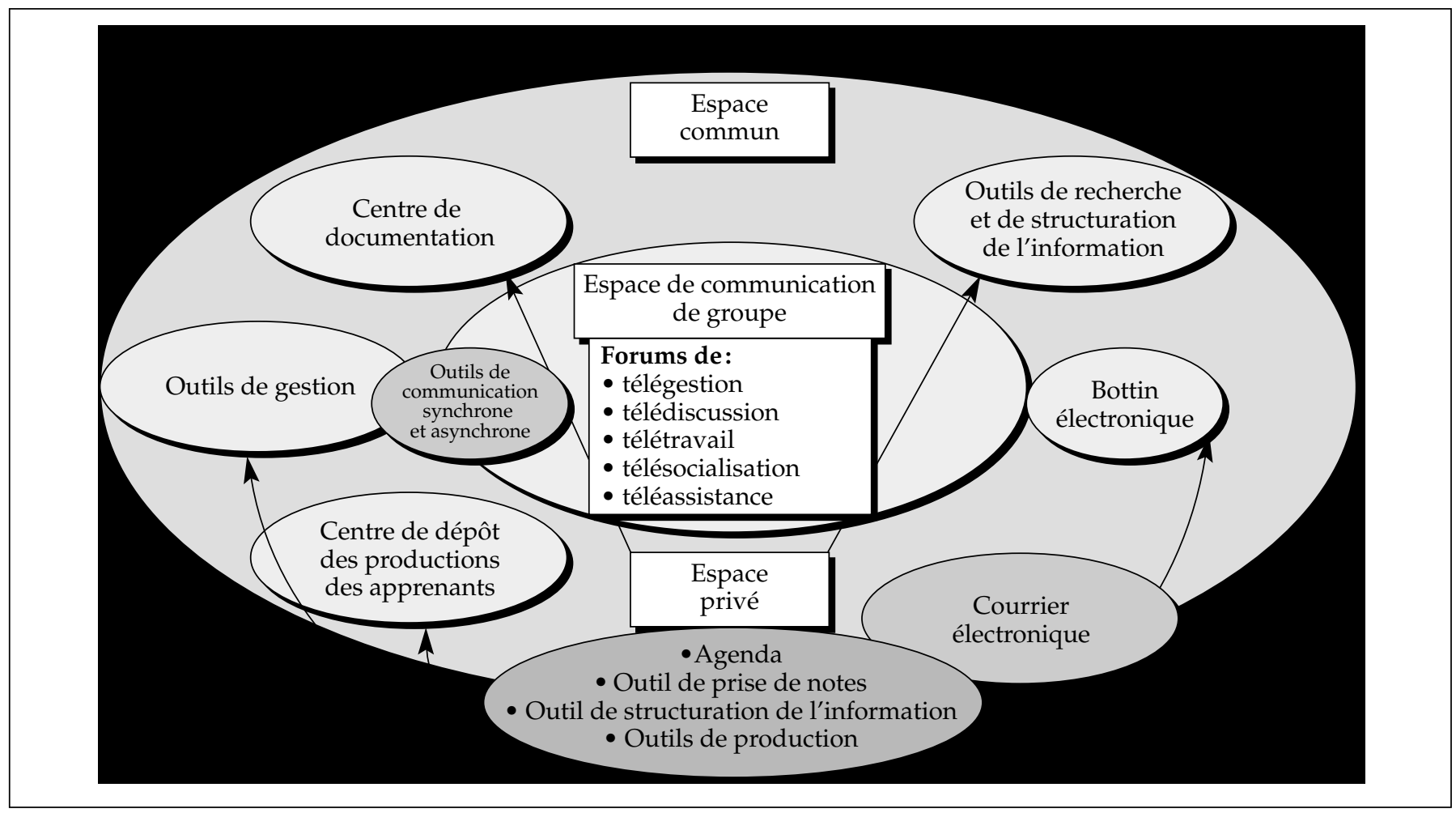


collaboration toute entité que le groupe peut partager et qui permet de traiter, de traduire ou de rendre compte des représentations élaborées individuellement ou en groupe. Pour les collaborateurs, ces espaces sont des ressources ou des outils porteurs de cognition. Ce sont de véritables partenaires de la collaboration (Schrage, 1990).

Certains espaces de collaboration peuvent être prévus, mis en place et aménagés par les concepteurs d'environnements d'apprentissage. D'autres s'organisent au fil de la collaboration tels les univers intellectuels, culturels, sociaux ou affectifs qui sont des émanations plus ou moins spontanées du groupe que le concepteur peut difficilement prévoir et planifier. Pour les fins $\mathrm{du}$ design pédagogique des environnements d'apprentissage virtuels, nous proposons aux concepteurs de prévoir trois grands espaces de base pour la collaboration (voir figure 2.3):

- l'espace privé,

- l'espace commun,

- l'espace de communication.

\section{L'ESPACE PRIVÉ: UN CENTRE DE RÉALISATION DE LA TÂCHE D'APPRENTISSAGE}

Il peut sembler étrange de parler en premier lieu d'espace privé lorsqu'on traite de collaboration. Mais comme l'effort collectif se nourrit du travail de chaque membre du groupe, il faut que chacun dispose d'un endroit pour réaliser certaines activités et pour se préparer à les partager avec le groupe.

L'espace privé est celui que chacun se construit lui-même, à sa manière, sur son poste de travail. Selon ses besoins et ses préférences, l'apprenant y installe des outils, crée des routines de travail, organise et gère les objets qu'il a choisis d'y mettre. Il pourrait lui être possible d'éclater son espace privé pour le prolonger sur un serveur distant où il pourra archiver des documents ou encore traiter des données ou de l'information à l'aide de systèmes qui ne peuvent résider sur son poste. Le concepteur verra donc à rendre disponibles dans l'environnement tous les outils et les ressources dont l'apprenant pourra avoir besoin dans son espace privé. 
Essentiellement dédié aux travaux personnels de recherche d'information, réflexion, d'appropriation et de production, l'apprenant travaille dans l'espace privé pour organiser sa pensée et ses idées, élaborer ses représentations, faire le point sur ses connaissances, préparer ses interventions et travailler à la production de documents qu'il partagera avec le groupe. À partir de cet univers personnel, il doit pouvoir accéder à tous les autres espaces et à toutes les ressources de l'environnement. Le passage d'un espace à l'autre ou d'une ressource à l'autre doit être transparent. Au cours d'une séance de travail, l'apprenant doit pouvoir se déplacer aisément vers l'espace de communication pour rejoindre d'autres apprenants ou vers l'espace commun afin de rechercher de l'information, d'importer un outil logiciel, d'y travailler à partir du serveur, d'y déposer le produit d'un travail ou $d^{\prime} y$ consulter les productions de ses pairs.

\section{L'ESPACE COMMUN : UN CENTRE DE RESSOURCES}

L'espace commun est un véritable centre de ressources polyvalent et accessible à tous. Il contient divers outils: des matériels pédagogiques, didactiques, méthodologiques, des sources d'information variées, des logiciels, etc., et rassemble donc les matériels et les logiciels qui nourriront l'apprentissage et qui serviront à bâtir ou à enrichir l'espace privé. En outre, l'espace commun est le lieu où les apprenants viennent déposer leurs productions. Accessibles à tous, ces productions seront partagées avec le groupe et pourront être annotées et commentées à l'aide d'outils logiciels disponibles dans l'environnement.

Les outils logiciels que l'on retrouve dans l'espace commun peuvent être, par exemple, des logiciels de planification, de gestion, de production écrite et multimédia, de communication, de recherche d'information, d'aide à la structuration de ses idées et à la représentation des connaissances. Les outils pédagogiques, didactiques, méthodologiques peuvent prendre la forme de guides d'étude, de présentations de stratégies d'apprentissage, de consignes de travail, de didacticiels, etc. Les sources d'information présentes dans l'environnement peuvent être aussi fort nombreuses également: des documents textuels ou multimédias, préparés expressément pour la formation en cours, des recueils 
de documents déjà publiés, des bases de données, des bibliographies, des webographies qui guident les apprenants dans leur recherche d'information sur le Web, etc. Ces ressources peuvent être logées sur plusieurs serveurs, mais il importe que l'accès soit toujours transparent pour l'apprenant.

\section{L'ESPACE DE COMMUNICATION DE GROUPE: UN CENTRE DE NÉGOCIATION ET DE VALIDATION DES CONNAISSANCES}

La collaboration est faite d'interaction et de dialogue. La communication y domine; elle en est le cœur. De cela découle l'importance à donner à l'espace de communication dans les environnements d'apprentissage. C'est l'endroit où les apprenants se rencontrent, c'est le lieu où ils se parlent. $C^{\prime}$ est là où le groupe se forme, où sa dynamique se cristallise. C'est l'espace qui permet au groupe d'exister et de vivre. Plus loin, nous analyserons de manière détaillée les caractéristiques de cette communication et nous présenterons les outils qui servent à la médiatiser et à la soutenir. Disons simplement pour l'instant que, dans cet espace, les apprenants communiquent en modes synchrone ou asynchrone, en langue naturelle, qu'elle soit écrite ou parlée.

L'espace de communication comprend des outils synchrones et asynchrones. Les plus couramment utilisés sont le courriel, le forum électronique, le babillard, le bavardage (ou chat) et les tableaux blancs. Parmi cet éventail d'outils de communication, le forum électronique est, pour nous, le plus important. Il l'emporte haut la main sur les autres outils pour soutenir l'apprentissage collaboratif à distance. Parce qu'il fonctionne en mode asynchrone, le forum rassemble les apprenants quelles que soient les contraintes de temps et d'espace. Il focalise les communications en permettant à tous et chacun de se faire entendre et fournit au groupe des lieux pour vivre, interagir, travailler et socialiser.

Avant d'analyser en profondeur la communication dans le cadre des forums électroniques, nous verrons d'abord l'importance de la communication pour la collaboration et l'apprentissage. 


\section{COMMUNICATION, COLLABORATION ET APPRENTISSAGE}

Collaborer et apprendre sont essentiellement des actes de communication qui procèdent par le dialogue et la conversation. Ils sont marqués par la bienveillance, l'ouverture et la générosité. Ce sont des gestes vrais dont il faut arriver à préserver le caractère authentique même s'ils sont médiatisés. Dans les pages qui suivent, nous tenterons de mettre en lumière le fonctionnement de cette communication médiatisée afin de pouvoir réunir les conditions qui lui sont favorables dans un environnement de collaboration.

\section{LA CONVERSATION AU CCEUR DE LA COLLABORATION}

Une étude menée auprès de groupes de chercheurs a permis de décrire la collaboration intellectuelle et les comportements des collaborateurs et de comprendre l'importance de la conversation dans ce processus. Schrage (1990) a observé que les collaborateurs intellectuels se réunissent pour partager leurs idées, communiquer leur vision des choses, élaborer des hypothèses, discuter et s'entendre sur l'orientation à donner au travail. Au cours des échanges, ils tentent de concilier les points de vue de chacun et de dégager un schéma rassembleur. Ils s'entendent sur le but de la collaboration et sur le processus de travail. Les collaborateurs ne sont toutefois pas toujours d'accord et ils n'ont pas à l'être. Ils peuvent s'opposer ou même s'affronter dans le respect les uns des autres. Obtenir un consensus sur toutes les questions n'est pas une règle absolue. Au contraire, la collaboration est d'autant plus saine et productive qu'elle permet l'expression d'idées et de points de vue divergents, même opposés. À cet égard, chaque groupe développe un seuil de tolérance au désaccord, mais s'il le dépasse, la collaboration prend fin.

Pour les collaborateurs, le travail en groupe alterne avec le travail individuel. Après leurs rencontres, ils partent chacun de leur côté pour continuer à travailler. Ils se réuniront à nouveau, de manière formelle ou informelle, à une fréquence et à un rythme convenus, pour rendre compte de l'avancement de leurs travaux, de leurs découvertes et de leurs réflexions. Les contributions de 
chacun seront analysées, commentées, critiquées et évaluées en fonction des résultats visés (Suthers et Weiner, 1995 ; Steeples, Goodyear et Mellar, 1994 ; Shedletsky, 1993 ; Rowntree, 1995).

Schrage explique que c'est en conversant que les collaborateurs se rejoignent et que c'est grâce à la conversation que la collaboration se construit. Lorsqu'ils se parlent, l'intention tacite des collaborateurs est d'apprendre des autres, c'est-à-dire comprendre ce que les autres expriment, mais aussi se comprendre soi-même en relation avec les autres. La conversation a une fonction de médiation; elle soutient la négociation qui vise à rapprocher les pensées et à réduire l'écart entre l'état des connaissances de chacun. À travers elle, les collaborateurs s'adaptent les uns aux autres, ils arrivent à expliciter leurs connaissances, à s'approprier celles des autres, à faire émerger de nouveaux points de vue, à développer une orientation commune et à s'entendre sur une vision partagée du travail à accomplir. Le défi des collaborateurs consiste à négocier l'arrimage des points de vues de chacun et à faire émerger un schème collectif qui orientera les travaux. Dans cette démarche, la conversation est le lieu central de partage et de négociation, le lieu de convergence du processus de collaboration (Bouton et Garth, 1983; Lemke, 1994 ; Cole, 1993 ; Fisher, 1995; Guzdial et al., 1995).

\section{LA CONVERSATION COMME MODÈLE D'APPRENTISSAGE}

Les collaborateurs apprennent les uns des autres. Il peut en être ainsi d'apprenants autonomes et responsables. C'est ce que Laurillard (1995) propose en développant à l'intention des étudiants universitaires un modèle d'apprentissage basé sur la conversation. Ce modèle, qui convient particulièrement bien à l'apprentissage des adultes, reconnaît que l'acquisition de connaissances relève d'une démarche sociocognitive. Il s'adresse aux apprentissages complexes et il est conçu pour soutenir l'émergence de connaissances abstraites. En s'appuyant sur Linard (1995), Laurillard entend les connaissances abstraites comme celles qui vont au-delà des connaissances pragmatiques et de premier niveau, issues du bon sens, des perceptions, des représentations et de l'action. Son modèle conversationnel oriente et 
structure les échanges entre formateurs et apprenants. Il s'articule autour de quatre grandes fonctions, présentes dans la collaboration, qui correspondent à quatre phases de l'apprentissage.

- Pour le formateur, la fonction discursive aide à repérer l'écart entre le modèle de connaissances des apprenants et le modèle de connaissances de l'expert $^{2}$; elle sollicite l'expression des conceptions, des visions et des points de vue de chaque apprenant. Formateur et apprenants s'emploient ainsi à dégager un schème ou un modèle collectif de connaissances.

- La fonction adaptative vise l'adaptation par le formateur de ses interventions à l'état des connaissances des apprenants.

- La fonction d'interaction fournit aux apprenants une rétroaction sur les résultats des expériences concrètes et pratiques qu'ils auront menées pour appliquer et pour manipuler les objets de connaissances. Elle sous-entend la mise en commun des travaux accomplis individuellement et des résultats obtenus.

- La fonction réflexive s'applique à l'ensemble du processus but-action-rétroaction dans l'intention de faire émerger de nouvelles connaissances à partir de ce que les apprenants ont découvert aux divers moments de l'apprentissage. Cette fonction vise à produire des connaissances de second niveau, abstraites et décontextualisées, telles que principes, concepts, méthodes, etc.

Le modèle de conversation en est un qui convient au processus de collaboration. Il nous rappelle que la réflexion et le retour sur la démarche des apprenants sont essentiels à l'apprentissage ; qu'ils permettent aux apprenants de prendre du recul, d'objectiver les nouvelles connaissances et d'évaluer le processus d'acquisition de ces connaissances.

2. Voir à ce sujet le chapitre 4 qui porte sur la dynamique cognitive de l'apprentissage collaboratif. 


\section{LA COMMUNICATION DE GROUPE SYNCHRONE OU ASYNCHRONE}

Cette étude de la conversation comme pratique de communication pour collaborer et pour apprendre nous amène à réfléchir sur les outils les mieux adaptés pour médiatiser ce genre de communication. Nous nous penchons ici sur les avantages et désavantages des outils synchrones et asynchrones.

Les outils de communication synchrone ${ }^{3}$ permettent à des personnes éloignées de communiquer en temps réel. Dégagées de la contrainte spatiale, elles doivent se plier à un horaire convenu pour se rencontrer. Ces systèmes sont conçus pour une communication immédiate. Ils sont très appréciés des groupes pour apprendre à se connaître et à socialiser. Ils sont efficaces pour prendre des décisions rapides, pour coordonner les activités et pour offrir des conseils et de l'aide technique (de la Teja, Lundgren-Cayrol et Paquin, 1997). Ces rencontres virtuelles sont de courte durée et ont un caractère éphémère. Elles se prêtent bien aux échanges qui doivent mener à un résultat immédiat. Bien que la plupart des outils synchrones permettent de garder en mémoire la trace de la rencontre et de la consulter une fois l'événement terminé, ils n'ont pas été conçus à cette fin.

Par ailleurs, la communication asynchrone libère des contraintes et de temps et d'espace. Au moment qui leur convient, $\mathrm{du}$ jour comme de la nuit, les participants se joignent au groupe pour échanger, discuter, converser. La durée de ces rencontres virtuelles peut s'étendre sur des semaines, des mois, voire des années. Les participants disposent de temps pour laisser mûrir les idées. À cet égard, notre travail auprès de groupes d'apprenants à distance nous a permis d'observer que les échanges

3. On pense ici à la vidéoconférence et aux nombreux logiciels de «chat» maintenant disponibles sur le Web qui offrent un éventail de fonctionnalités telles que la liste des présences, le multifenêtrage, le partage de document, le tableau blanc, l'appel de sites URL, le courrier électronique, etc. Voir par exemple les logiciels Netmeeting, ICQ, le module Conference dans Communicator 4 de Netscape, Webex, etc. 
asynchrones sont souvent nettement plus réfléchis, mieux structurés et plus profonds que les échanges synchrones. Car avant de faire leurs interventions, les apprenants peuvent relire, analyser et mieux comprendre les contributions des autres. Les messages étant stockés, ils peuvent être consultés à tout moment. La trace de la discussion, accessible en permanence, aide les apprenants à comprendre l'évolution de leur travail et le sens à lui donner. En outre, pour le groupe, l'analyse de cette trace se révèle un excellent moyen de faire un retour sur son fonctionnement et d'en tirer des pistes pour mieux collaborer.

Certains penseront que la communication asynchrone sera bientôt supplantée par la communication synchrone jugée plus naturelle et plus conviviale et dont on observe la popularité grandissante pour les échanges de groupe par télématique. Mais, selon nous, le mode asynchrone occupera toujours une place de choix dans les démarches de l'apprentissage à distance. Les atouts de l'asynchronicité sont indéniables pour la collaboration et pour l'apprentissage à distance en raison de la souplesse des échanges qu'il procure et de la profondeur du contenu qu'il permet d'atteindre. Comme nous espérons le montrer ici, il donne lieu, dans le cadre des forums électroniques, à la mise en œuvre de processus de collaboration novateurs que le mode synchrone ne nous a jamais permis de réaliser. Toutefois, pour que la communication asynchrone s'impose comme un véhicule d'apprentissage puissant et pour qu'elle devienne de plus en plus satisfaisante, il faut pouvoir offrir aux apprenants des outils plus performants que ceux qui existent actuellement pour médiatiser et soutenir la collaboration avec une efficacité renforcée.

\section{L'INTERACTION, ÉCRITE ET ASYNCHRONE}

Dans les environnements virtuels qui nous intéressent, les interactions humaines jouent un rôle de premier plan. Ce n'est pas l'image qui y domine, mais plutôt l'écrit qui sert de véhicule et d'outil privilégié pour soutenir les activités de construction des 
connaissances. Cela explique l'usage incontournable des forums électroniques dans les environnements d'apprentissage collaboratifs. Dans cette partie, nous voulons montrer que si cette technologie est largement utilisée pour la collaboration, ce n'est pas par défaut ou parce qu'elle est à la portée de tous, mais bien parce qu'elle recèle des potentialités pédagogiques appréciables.

\section{LE FORUM ÉLECTRONIQUE ET AUTRES TECHNOLOGIES DE COMMUNICATION ASYNCHRONE}

Quelle différence faire entre le forum électronique (souvent appelé forum de discussion), le courriel, le babillard et les collecticiels? Ne sont-ils pas des outils qui se ressemblent et qui servent aux mêmes usages? Le courriel ne permet-il pas d'envoyer des messages à des groupes tout comme le forum électronique et le babillard? Qu'est-ce qui distingue ces outils? Comme ils sont souvent intégrés dans un même système et qu'on peut facilement naviguer de l'un à l'autre de manière transparente, il n'est pas facile de se rendre compte qu'ils induisent une dynamique de communication fort différente. Une chose est certaine, ils sont tous au service des groupes et de la communication humaine. Mais chacun de ces outils remplit une fonction différente. Nous les décrirons séparément pour mieux cerner leurs différentes fonctions.

Les collecticiels sont des outils construits pour faciliter la manipulation d'information à partir de bases de données utilisant une ou des interfaces programmables. Fraase (1991) les définit ainsi: "Groupware is about people managing together their collective information resources» (p. 47). Ce sont des outils de productivité de groupe qui ont été développés pour les entreprises. Ils facilitent l'organisation et l'accès à des informations destinées à des groupes qui doivent réaliser collectivement une tâche ou un projet. Ces systèmes permettent de manipuler et de traiter des informations se rapportant à la tâche, à sa gestion et à son mode de réalisation. Contrairement aux forums électroniques, les collecticiels n'ont pas comme objectif principal de soutenir le processus dynamique de la communication de groupe 
à distance, mais plutôt celui d'aider à traiter les informations et les décisions qui résultent de discussions et à les mettre à la disposition du groupe.

Les babillards, ou outils de communication pour groupes d'intérêt, sont construits selon le principe du tableau d'affichage (Gunawardena, 1991a et 1991b). Utilisés le plus souvent par un ensemble de personnes qui partagent un intérêt commun, ils permettent l'inscription d'information dans un «lieu» accessible à ces personnes. La principale fonction des babillards est de faciliter la circulation de l'information peu importe la tâche ou le projet communs à réaliser. Les usagers de ces systèmes ne sont pas des groupes de collaborateurs. Ils forment des rassemblements de personnes poursuivant des démarches personnelles qui ne se rattachent pas à une démarche collective.

Les forums électroniques ont été conçus pour faciliter les interactions humaines et pour encadrer la dynamique des groupes. Leur architecture, leurs fonctionnalités et leurs interfaces invitent les usagers à adhérer au groupe, à s'engager envers celui-ci, à collaborer et à participer activement à ses activités. Les observateurs silencieux (lurkers), qui fréquentent souvent les babillards, n'ont pas leur place dans un forum. L'interface presse les usagers de participer. Presque chaque geste, presque chaque clic appellent une action ou une décision relative à la participation. Après la lecture de chaque message, l'usager est invité à réagir. "Voulezvous rédiger un message? " Voulez-vous répondre? « Voulezvous faire un commentaire?» "Voulez-vous transférer le message dans un autre forum ? »... La dynamique des échanges est constamment stimulée. Au début de chaque message, les participants indiquent l'objet et signalent le lien entre le message qu'ils rédigent et les messages antérieurs. C'est un encouragement à la pertinence et à la cohérence. À tout moment, il est possible d'afficher les messages précédents pour reconstituer la trame de la discussion; c'est un encouragement à l'élaboration d'une argumentation. Au fil des messages, des liens se tissent entre les idées et aussi entre les participants. Lorsque les échanges sont menés par un bon modérateur et que le but est significatif, 
les habitués des forums électroniques ont toujours la surprise d'observer l'intensité des rapports qui s'établit entre les participants, la richesse de l'information issue de la conversation et du partage des idées (Harasim, 1990 ; Hiltz, 1987). Les concepteurs de systèmes de forums électroniques reconnaissent la nécessité de l'intervention d'un modérateur pour assurer l'efficacité du fonctionnement du groupe. C'est pourquoi des droits exclusifs lui sont attribués pour organiser et gérer les échanges du groupe.

Les systèmes de forums électroniques permettent à un groupe donné d'aménager son propre espace de communication en faisant de chaque forum un lieu à vocation unique et spécifique. Certains forums pourront être réservés à la vie sociale du groupe, d'autres à la planification du travail, et d'autres encore à la réalisation des tâches et à la coordination des efforts. Il est toujours possible pour un groupe en cours de travail de revoir l'aménagement de ses forums. On peut fragmenter un thème jugé soudainement trop vaste en plusieurs sous-thèmes et créer pour chacun un nouveau forum électronique; on peut réorganiser les forums pour traiter un nouveau sujet ou disposer d'une question jugée importante par le groupe; on peut également changer la vocation d'un forum ou en ajouter un à l'environnement pour couvrir une fonction qui a émergé et qui n'apparaissait pas dans le plan de départ.

La navigation entre les forums réservés à un groupe est généralement transparente. Dans plusieurs systèmes, on peut passer de manière automatique d'un forum à un autre, ce qui incite les membres à prendre connaissance de tous les échanges sur tous les thèmes qui préoccupent le groupe.

Collecticiels, babillards et forums sont des outils de collaboration, apportant chacun une contribution spécifique au travail de groupe. Notre intérêt pour le forum vient du fait qu'il peut répondre, plus que les autres outils, à un large spectre de besoins chez les groupes collaboratifs et parce qu'il propose un mode inédit de communication humaine (tableau 2.2). 
Tableau 2.2

\section{LE FORUM ÉLECTRONIQUE}

\begin{tabular}{|c|c|}
\hline $\begin{array}{l}\text { Le forum } \\
\text { électronique }\end{array}$ & ... pour l'apprenant \\
\hline Qu'est-ce que c'est? & $\begin{array}{l}\text { - C'est un lieu virtuel où se construit le discours d'un } \\
\text { groupe, où l'apprenant s'approprie de nouvelles } \\
\text { connaissances en conversant avec d'autres. } \\
\text { - C'est une agora de collaboration et de socialisation. }\end{array}$ \\
\hline Que permet-il? & $\begin{array}{l}\text { - On peut y exposer des idées, élaborer sa pensée, construire } \\
\text { de nouvelles connaissances, les valider et les confirmer } \\
\text { avec le soutien du groupe. } \\
\text { - Il facilite l'apprentissage de connaissances complexes } \\
\text { appartenant à des domaines peu ou mal structurés. } \\
\text { - Il favorise une attitude réflexive sur l'apprentissage. } \\
\text { - Il permet à un groupe de vivre une expérience } \\
\text { d'apprentissage motivante et de développer un } \\
\text { engagement sociocognitif qui donne encore plus de sens } \\
\text { à l'apprentissage. }\end{array}$ \\
\hline Qui participe? & $\begin{array}{l}\text { - Des agents humains qui créent un réseau social: le tuteur } \\
\text { ou le formateur et d'autres apprenants qui travaillent au } \\
\text { sein de petits groupes (collaboration spontanée, informelle) } \\
\text { ou au sein d'un grand groupe (collaboration encadrée, } \\
\text { formelle). } \\
\text { - Des agents machines qui composent l'environnement } \\
\text { technologique: des outils de collaboration, de coordination } \\
\text { et de soutien aux processus cognitifs et sociaux. }\end{array}$ \\
\hline $\begin{array}{l}\text { Comment } \\
\text { participe-t-on? }\end{array}$ & $\begin{array}{l}\text { - Des agents humains transmettent des messages au groupe ; } \\
\text { l'interaction est la sémantique de ces messages. } \\
\text { - Des agents machines, greffés sur le forum, offrent un } \\
\text { support automatisé à l'apprentissage en fournissant, par } \\
\text { exemple des représentations multiples de ce qui est dit } \\
\text { dans les messages; ce support est immédiat, rapide, } \\
\text { interactif. }\end{array}$ \\
\hline $\begin{array}{l}\text { Quand convient-il } \\
\text { de l'utiliser? }\end{array}$ & $\begin{array}{l}\text { - Lorsque l'apprentissage met en jeu des compétences de } \\
\text { haut niveau comme l'analyse, la synthèse et l'évaluation, } \\
\text { le forum est un choix pédagogique approprié. Il est alors } \\
\text { conçu comme un environnement de résolution de } \\
\text { problèmes, de prise de décision. Il témoigne de } \\
\text { l'intentionnalité de la situation d'apprentissage. } \\
\text { - Dans un cadre formel d'apprentissage, lorsque les } \\
\text { apprenants sentent qu'ils ont besoin des autres pour } \\
\text { apprendre, lorsqu'un groupe de } 15 \text { à } 30 \text { apprenants veulent } \\
\text { apprendre ensemble avec le soutien d'un formateur et } \\
\text { lorsqu'ils sont disposés à s'entendre pour atteindre un } \\
\text { objectif partagé. } \\
\text { - Dans un cadre informel, lorsqu'un nombre suffisant de } \\
\text { personnes souhaitent former un groupe et travailler } \\
\text { ensemble pour apprendre. }\end{array}$ \\
\hline
\end{tabular}




\section{UN MODE INÉDIT DE COMMUNICATION HUMAINE}

Le forum réunit des groupes dans un espace virtuel et leur propose de communiquer en mode conversationnel. Ces conversations évoluent comme celles de collaborateurs qui, au fil de leur propos, développent des idées nouvelles. Dans un forum, chacun jouit du droit de parole et tous sont invités à exprimer leurs idées et à réagir à celles des autres. L'alternance des messages n'est pas soumise au tour de parole de la conversation verbale. Les règles d'étiquette et le code de comportement de la conversation orale ne s'appliquent pas. Les participants d'un forum sont libérés de l'ordre séquentiel de la prise de parole et de la règle de politesse qui demande aux interlocuteurs de parler un à la fois et de répondre à ce qui vient d'être dit (Davie et Wells, 1991; Duning, 1993 ; Donovan, 1995 ; Eales et Welsh, 1996 ; Edelson et al., 1995). Il est possible, à tout moment, de faire part de points de vue différents, d'idées divergentes ou même controversées. D'un message à l'autre, le contenu n'a pas à être en rapport direct avec celui qui précède. Il peut répondre à un message qui apparaît beaucoup plus haut à l'écran. Le désordre qu'on peut observer dans la transcription chronologique d'un forum électronique n'est qu'apparent puisque des fonctionnalités permettent de retracer les liens entre les messages. Ainsi, certaines commandes permettent de regrouper les messages portant sur une même idée ou un même objet et de reconstituer le déroulement d'un raisonnement ou d'une argumentation.

L'asynchronicité du forum électronique offre de nombreux avantages par rapport au caractère éphémère de la conversation de vive voix. Au cours d'un échange synchrone, chacun livre des informations, fait valoir son point de vue et réagit spontanément à ce qui vient d'être dit oubliant parfois ce qui a été mentionné au cours des minutes qui précèdent. Une bonne idée peut être perdue, mal comprise ou déformée sans qu'on puisse la reprendre ou la clarifier nuisant ainsi à la collaboration (Burge, 1994; Davie et Wells, 1991). Ni la mémoire des collaborateurs ni la prise de notes ne peuvent rendre compte de manière authentique et objective du déroulement des conversations. Dans un forum, les idées ne s'évanouissent pas. Les messages sont mis en mémoire et deviennent des objets tangibles qui pourront être manipulés, 
placés dans une base de données, annotés, commentés, critiqués, restructurés, etc. Rien de ce qui s'est dit ne se perd et toutes les idées peuvent être récupérées à tout moment dans leur intégralité.

Le forum se prête remarquablement à l'expression libre de points de vue concurrents et à l'élaboration de plusieurs raisonnements en parallèle. Les fonctions d'étiquetage et de tri des messages permettent de remonter le fil des idées. Il peut donc y avoir, au sein des groupes, un réel partage, un apprentissage mutuel et l'émergence d'une collaboration véritable (Pugh, 1993; Salomon, 1992). Dans un contexte d'apprentissage, le mode conversationnel propre au forum peut être exploité pour soutenir le modèle d'apprentissage proposé par Laurillard que nous avons évoqué précédemment et que nous reprenons brièvement ici en adoptant le point de vue de l'apprenant.

- La fonction discursive, qui permet de faire émerger l'état des connaissances, s'exerce grâce à la possibilité qu'ont les apprenants d'exprimer des points de vue divers et d'élaborer en parallèle une série de raisonnements sur un thème ou sur un sujet donné.

- La fonction adaptative est assurée par la mise en mémoire des messages et la possibilité de les consulter à tout moment. Le formateur peut, en relisant les messages, dégager un schème ou un modèle collectif de connaissances en fonction duquel il orientera ses interventions et la suite des apprentissages.

- Le forum est au service de la fonction d'interaction et de la rétroaction. Après avoir travaillé individuellement, après avoir manipulé, appliqué et expérimenté des objets de connaissances, les apprenants se retrouvent dans les forums pour faire part des résultats obtenus et pour recevoir les commentaires et les rétroactions du formateur et du groupe.

- La fonction réflexive vise le retour sur le processus de collaboration, l'extraction des connaissances de second niveau et l'objectivation. Elle est facilitée par la mise en mémoire des messages qui offre la possibilité de revoir la transcription des échanges, d'analyser les comportements du groupe et d'évaluer tant sa production que sa productivité. 


\section{LES LIMITES COGNITIVES}

On peut dire du forum électronique, textuel et asynchrone, que c'est un outil souple qui facilite la collaboration à distance et qui favorise l'apprentissage. Son fonctionnement - basé sur le modèle conversationnel et la communication asynchrone - et ses caractéristiques technologiques - qui permettent la création de lieux à vocations spécifiques, la récupération de la trace des échanges et le tri des messages - soutiennent les processus sociaux et cognitifs des groupes de travail (Davie et Wells, 1991 ; Wells, 1995 ; Burge et Roberts, 1993 ; Cerratto et Belisle, 1995; Bruce et al., 1993; Crotty, 1994). Toutefois, force nous est de constater que les systèmes de forums dont nous disposons actuellement imposent des limites à la mise en œuvre de ces processus.

\section{Limite de la présence sociale}

La présence sociale ne s'exprime que faiblement dans un forum électronique. L'absence d'indices paraverbaux (ton, attitude, gestuelle, etc.) peut causer de l'incertitude chez les participants. Certains n'arrivent pas à savoir s'ils sont acceptés par le groupe ou à percevoir quelle est leur place au sein du groupe. Il est alors difficile pour eux de s'engager dans la collaboration et de développer un sentiment d'appartenance (Burge, 1994). En outre, l'absence de pression sociale n'a pas toujours un effet positif sur la collaboration. Elle augmente le sentiment de liberté personnelle et peut encourager l'individualisme qui, s'il se développe, menace la cohésion et la productivité du groupe. Les modérateurs de forums sont généralement bien au fait de ces limites. Ils savent utiliser des stratégies pour contrer les effets négatifs d'une faible présence sociale. En revanche, ils connaissent peut-être moins bien les limites cognitives des forums électroniques et, en conséquence, maîtrisent moins les stratégies de soutien au développement de la pensée. 


\section{Limite du processus de développement de la pensée}

Dans un forum, les fonctions d'étiquetage et de tri permettent de regrouper les messages se rapportant à un même sujet et de retracer l'évolution de la conversation et le développement des idées. Cette représentation de la discussion, linéaire à souhait, ne traduit pas le processus récursif du développement de la pensée. Lorsque la pensée se construit, les idées ne viennent pas à l'esprit dans un ordre, une structure ou une formulation finale. Que ce soit pour élaborer un raisonnement ou développer un concept, pour bâtir une argumentation ou pour développer le fil conducteur d'une pensée, les idées s'ordonnent et se précisent de manière récursive. Il faut de nombreux retours sur la pensée pour réviser, clarifier, expliciter, élaborer ou structurer ce qui a été imaginé, dit ou écrit. Ce processus n'est pas linéaire et ne procède pas toujours selon une logique évidente. Il dépend de la spontanéité du penseur, de sa créativité, de son profil cognitif et de son organisation mentale.

Le texte d'un forum est plat et linéaire ; il n'a qu'une dimension et ne propose qu'une lecture séquentielle. La suite de messages que l'on déroule à l'écran ou que l'on imprime apparaît déroutante surtout lorsqu'on s'intéresse aux processus de la pensée et qu'on souhaite en observer l'évolution. La simple transcription d'un forum ne réussit pas à traduire clairement la structure élaborée par le groupe. La présentation du contenu des messages ne rend pas compte du résultat de l'activité cognitive du groupe ni ne traduit l'exercice de pensée à laquelle les participants se sont livrés.

Nous demandons aux systèmes de forums qu'ils offrent plus que ce pourquoi ils on été conçus. Nous voulons qu'ils engendrent des représentations des processus cognitifs du groupe (raisonnements spiralé, arborescent, moléculaire, circulaire, latéral, multidimensionnel, etc.; processus de révision, comparaison, restructuration, etc.) alors que c'est plutôt le stockage des interventions qu'ils visent. Afin de mieux servir la communication de groupe et l'apprentissage collaboratif, ces systèmes gagneraient à intégrer des fonctionnalités qui permettent d'augmenter leurs capacités de représentation en montrant, par des formes 
plus dynamiques, plus visuelles ou symboliques, les cheminements aboutissant aux constructions mentales. Les développeurs pourraient y arriver dans un premier temps en greffant aux systèmes de forums des outils de type remue-méninges, carte conceptuelle, hypertexte, etc., qui proposent une visualisation non linéaire d'un contenu. Il y aurait sans doute au début des manipulations à faire sur le texte pour en assurer le traitement par ces outils, mais l'exercice en vaudrait la peine dans la mesure où il servirait à établir le bien-fondé de nos demandes. C'est dans cette perspective que des chercheurs du LICEF (Ricciardi Rigault, 1995) s'emploient actuellement à développer des outils d'analyse du texte qui peuvent se greffer aux forums. Ils permettent de faire émerger des structures autrement invisibles et $\mathrm{d}^{\prime}$ en fournir une image dynamique ${ }^{4}$. Les formateurs et les apprenants pourraient ainsi observer les représentations qu'ils produisent, ce qui devrait favoriser une certaine emprise sur leurs compétences cognitives.

Malgré ses limites, il ne faut pas délaisser le forum comme outil de soutien à l'apprentissage collaboratif. Il demeure malgré tout une des ressources les plus efficaces pour la construction collective de connaissances.

\section{Limite du processus réflexif}

Le stockage de la trace est évoqué comme l'une des forces du forum électronique. En consultant l'historique des échanges, un apprenant peut à tout moment revoir le contenu des messages et prendre le temps de réfléchir à la dynamique dans laquelle le groupe est engagé. En analysant les messages, il peut aussi mieux comprendre le sens d'une discussion et mieux cibler ses interventions. Toutefois, on constate que le retour sur la trace n'est pas une pratique courante chez les participants. Les systèmes de forums en sont en partie responsables puisqu'ils encouragent rarement les participants à remonter dans les messages précédents avant d'intervenir. Les commandes qui apparaissent à la fin des messages invitent à réagir immédiatement, en ligne, sans

4. Il s'agit de la technologie Nomino qui a donné lieu à des expériences convaincantes d'analyse de messages. 
plus de réflexion. En outre, comme il n'est pas obligatoire de déclarer le lien entre un nouveau message et les anciens, les digressions et les points hors d'ordre sont monnaie courante dans les forums. C'est alors qu'il faut que le modérateur fasse preuve de beaucoup d'adresse pour garder le groupe sur les rails.

Bien que les systèmes de forums offrent aujourd'hui certaines fonctionnalités pour exploiter l'histoire de la conversation, cellesci ne sont pas évidentes et elles sont généralement peu utilisées par les apprenants. Il faut s'en remettre au modérateur pour proposer aux participants de le faire. Celui-ci peut également soutenir le processus réflexif en incitant les apprenants à ne pas répondre spontanément en ligne mais plutôt à prendre le temps de réfléchir avant de rédiger leurs messages hors ligne. En outre, en leur demandant d'indiquer à quel message ils répondent et le lien à établir, le modérateur encourage le processus réflexif. La réflexion et l'exploitation du contenu des messages constituent une discipline intellectuelle importante à acquérir. Il est à espérer que les futurs systèmes de forums électroniques offriront une aide à cet effet pour augmenter l'efficacité des échanges et soutenir le développement cognitif des usagers.

\section{Limites à la structuration des échanges}

Dans un environnement d'apprentissage collaboratif, chaque forum a une vocation spécifique. Le but, le contenu d'apprentissage, la tâche et le déroulement du travail y sont clairement identifiés. Il peut s'agir de réaliser des activités cognitives simples comme échanger de l'information sur un thème donné, ou d'activités plus complexes comme faire une étude de cas, prendre une décision, faire l'analyse d'une théorie, etc., ou encore d'activités à caractère social ou organisationnel. Il est regrettable que, pour réaliser une telle variété de tâches, les systèmes ne proposent qu'une seule et même structure. Tous les forums, quelle que soit la nature de l'activité à réaliser (Harasim, 1990; Hiltz, 1987) s'organisent et se déroulent de la même façon. On doit s'accommoder du même environnement et des mêmes fonctionnalités pour mener des tâches cognitives, réaliser des activités de socialisation et assurer des interventions de suivi et de coordination du travail. Comme si, à l'école, toutes les activités qui ont lieu 
dans le cadre de la vie scolaire (l'enseignement, la récréation, les laboratoires, le repas de midi, etc.) se passaient dans la même pièce en utilisant les mêmes ressources. Pour contourner ce problème, le modérateur doit planifier rigoureusement les diverses étapes et, par des consignes très précises, informer les apprenants des règles de participation qui s'appliquent à chaque forum; $c^{\prime}$ est un poids qui s'ajoute à sa tâche déjà lourde d'animateur psychosocial et de soutien cognitif et organisationnel.

\section{Limites du langage verbal et de la conversation}

Le forum, on l'a dit, utilise le langage verbal et adopte la conversation comme mode de représentation. Tout en reconnaissant la puissance de cette forme d'expression, il faut admettre qu'elle est rarement univoque et que l'ambiguïté inhérente aux conversations crée souvent de la confusion, ce qui nuit à la productivité et au bon fonctionnement des groupes collaboratifs. Par ailleurs, on sait que la collaboration vit et se maintient grâce à la diversité des apports et à la multiplicité des points de vue. Les systèmes de forums textuels ne devraient pas se limiter à la seule utilisation du langage verbal et $\mathrm{du}$ mode conversationnel. On aurait avantage à rechercher ceux qui permettent l'intégration ou la création de liens vers des formes de représentations multiples. Les apprenants devraient pouvoir recourir à d'autres outils qui permettent d'utiliser d'autres langages (mathématique, informatique, visuel, etc.) et de créer d'autres représentations (schémas, équations, images, modèles, etc.) qui pourraient être consultées, partagées et manipulées par le groupe dans l'espace commun. Ces outils de collaboration périphériques au forum et utilisés pour exprimer et communiquer ce qui ne peut être facilement expliqué par des mots assureraient que tous disposent de moyens adaptés à l'expression de leur forme de pensée.

Si nous insistons sur l'importance de pouvoir reproduire des représentations multiples, c'est que la collaboration véritable exige que chacun puisse s'exprimer, que tous soient capables de comprendre les contributions des autres et qu'ils saisissent collectivement le sens de la tâche à réaliser, du problème à résoudre ou de la question à étudier (Coleman et Furey, 1996; Bonk et King, 1995; Ehrlich et Cash, 1996 ; Hoadley et Hsi, 1993 ; Klemm et 
Snell, 1996 ; Kanselaar et Erkens, 1996 ; Prescott, 1994 ; Röscheisen, Mogensen et Winograd, 1996; Suthers et Weiner, 1995 ; Spuck, Prater et Palumbo, 1995 ; Dillenbourg, 2000).

\section{POUR UNE COLLABORATION FRUCTUEUSE}

En dépit de l'absence de lieu physique commun à partager, les apprenants réunis dans un forum découvrent une capacité d'action et de socialisation insoupçonnée. Une vie de groupe peut se créer et se développer dans l'espace virtuel. Des relations d'amitié se tissent, des conflits surgissent, des sentiments de découragement et d'enthousiasme se succèdent; l'humour, la contestation, le retrait se manifestent. Pour optimiser le succès de la collaboration, cinq facteurs humains doivent être considérés: la participation, la motivation, l'animation, la cohésion et la productivité du groupe (Lundgren-Cayrol, 1996).

\section{LA PARTICIPATION}

$C^{\prime}$ est en rédigeant et en transmettant des messages qu'on participe à un forum électronique ; la présence de lecteurs silencieux est mal tolérée. Chacun doit contribuer (Schrage, 1990). L'équation est simple: pas de messages, pas de participation; pas de participation, pas de forum. Le manque ou l'absence de participation peuvent avoir plusieurs causes que le concepteur de l'environnement ne peut contrôler:

- difficulté pour le participant d'accéder à un poste de travail ou à l'environnement informatique au moment désiré,

- habiletés technologiques déficientes,

- manque de temps pour communiquer,

- peu d'habiletés en écriture ou en lecture,

- manque d'attrait pour le langage verbal,

- manque de motivation,

- etc. 
En revanche, le concepteur peut encourager la participation en soignant l'aménagement de l'environnement et en faisant bien comprendre aux apprenants ses intentions pédagogiques et les règles de la collaboration.

\section{Établir les règles du jeu et développer une perception positive de la collaboration}

Dans sa démarche de design pédagogique, le concepteur choisit les ressources et les outils de l'environnement de collaboration. Il définit leurs usages et leurs fonctions. Il développe un rationnel pour justifier la tenue de un ou de plusieurs forums. Sa logique de conception l'amènera à déterminer la place des forums dans l'environnement et le rôle qu'ils auront à jouer dans l'apprentissage. Si, dans l'esprit du concepteur, la démarche d'apprentissage s'appuie sur des échanges et des interactions écrites, les forums occuperont alors une place centrale dans l'environnement.

Pour qu'un environnement d'apprentissage soit collaboratif, il ne suffit pas pour le concepteur de le déclarer tel et d'ouvrir un ou deux forums en invitant les apprenants à s'y joindre (Damphousse, 1996). Si l'on veut qu'une collaboration réelle se développe dans l'environnement, il faut faire en sorte que les forums ne deviennent pas que de simples lieux de bavardage fréquentés de manière occasionnelle pour répondre à des besoins individuels. Avant même le début de leur formation, les futurs apprenants doivent être informés du but de l'apprentissage et de sa finalité. On doit leur expliquer les principes de la collaboration, les exigences du travail en groupe, le rythme et la fréquence de la participation. Ces informations seront communiquées d'autant plus efficacement que le concepteur aura lui-même une vision et une compréhension profonde du sens de la participation et de l'usage réservé au forum dans une démarche de collaboration. Les apprenants qui décideront de s'engager dans une telle démarche le feront en toute connaissance de cause. Ainsi, on évitera à ceux qui n'ont aucun désir de s'engager d'être frustrés ou déçus par la formation. 
Il importe donc que l'apprenant conçoive de manière raisonnée le lien entre l'apprentissage collaboratif, la communication et la participation aux forums; qu'on lui rappelle que la collaboration s'appuie sur la poursuite d'un but commun, mais qu'elle doit également rejoindre ses intentions personnelles. Il doit aussi comprendre que, dans la démarche de formation qu'il a choisie, il sera aussi question d'apprendre à collaborer et que l'apprentissage de la collaboration fait partie des valeurs et des buts éducatifs auxquels le concepteur souscrit. L'apprenant doit, somme toute, développer une perception positive de la collaboration en y voyant une valeur ajoutée. Cette perception sera renforcée par les pratiques d'animation, par la rétroaction donnée à l'apprenant et par l'évaluation de l'apprentissage.

Une fois que les apprenants auront décidé de s'engager dans la formation et qu'ils auront accepté d'apprendre à collaborer, le but et les modalités de la participation pourront être discutés et négociés par le groupe : la cible commune, l'orientation du travail et l'organisation de la tâche d'apprentissage, les rôles, la fréquence des communications, l'échéancier, etc. Il faut se rappeler que sans consensus sur le but et les modalités de la participation, il est impossible de bâtir une communauté et de faire en sorte que les membres apprennent ensemble.

\section{LA MOTIVATION}

La collaboration est pragmatique; elle permet de satisfaire un besoin. Aux yeux des collaborateurs, elle n'a rien de théorique; elle revêt un caractère utilitaire, orienté vers la réalisation d'une tâche spécifique. C'est un moyen efficace d'atteindre un but et de tirer bénéfice de l'action collective. La collaboration est un phénomène raisonné, soutenu par des éléments de motivation extrinsèque et intrinsèque que l'on retrouve à des degrés divers chez les apprenants.

\section{La motivation extrinsèque}

La motivation extrinsèque s'installe lorsque l'apprenant réalise que la collaboration représente le moyen le plus efficace pour apprendre et pour atteindre le but qu'il poursuit. Un besoin 
s'impose alors : joindre les autres. Peu importe qui sont les autres collaborateurs, il s'engage sincèrement envers le groupe. L'apprenant s'adapte et s'accommode au groupe dans la mesure où il se sent compétent par rapport à celui-ci et où il a la conviction que le groupe peut l'aider dans la réalisation de la tâche d'apprentissage. Lorsque la motivation extrinsèque est installée, il ne perçoit pas la collaboration comme un piège. Il comprend que son engagement n'est pas permanent, que la collaboration est limitée dans le temps et qu'après la réalisation de la tâche elle prendra fin. Les relations interpersonnelles qui se développent au sein du groupe sont importantes pour lui, mais la collaboration ne leur est pas subordonnée.

La motivation extrinsèque s'intéresse surtout à la gestion des forces de chacun plutôt qu'à la recension des faiblesses, à la critique et aux doléances. Dans la mesure où chacun contribue en fonction de ses compétences, l'apprenant accepte ses collaborateurs même si, dans d'autres circonstances, il ne souhaite pas se retrouver avec eux. Il mise sur l'originalité et la spécificité des apports de chacun, sur l'interdépendance positive et sur la productivité du groupe pour l'aider à réaliser la tâche d'apprentissage.

Schrage (1990) explique que lorsque les collaborations prennent fin, les collaborateurs vivent à la fois un sentiment de tristesse et de libération. Ils sont tristes parce les personnes avec qui ils ont travaillé de manière si efficace et productive n'entreprendront pas d'autres travaux. Par ailleurs, ils se sentent libérés parce qu'un fois le travail terminé, les collaborateurs demeurent libres et ne se sentent aucunement obligés envers le groupe. L'idée d'une collaboration fructueuse pourra éventuellement inciter ces collaborateurs à faire à nouveau l'expérience de la collaboration.

\section{La motivation intrinsèque}

La motivation intrinsèque trouve sa source dans le plaisir d'interagir, dans le désir de prendre part à une vie de groupe et de s'engager, dans la soif de découvrir à travers le groupe le sens à donner à son apprentissage. Elle émane du besoin de réalisation de soi par la participation à une communauté d'apprentissage. L'apprenant perçoit alors la démarche collective comme une 
occasion de croître et de se transformer non seulement en intégrant des connaissances nouvelles, mais en développant ses capacités de collaboration et en apprenant à utiliser les technologies pour y arriver. La motivation intrinsèque peut être soutenue et renforcée par la réflexion sur les valeurs de la collaboration et sur le bénéfice qu'on retire d'en maîtriser les processus.

\section{L'ANIMATION DU GROUPE}

L'animation des forums électroniques est confiée à un modérateur. Il joue un rôle de référence capital dans le fonctionnement du groupe. Il choisit un style d'animation qui convient au profil du groupe afin de créer un climat propice à la collaboration, de favoriser la participation de chacun, de stimuler les interactions et d'assurer la régulation du déroulement du travail. Sa mission consiste à faciliter l'atteinte du but avec la plus grande efficacité possible (Damphousse, 1996). Il doit être capable de faire émerger une interdépendance positive entre les membres pour que chacun sente que ses expériences et ses contributions sont valorisées et enrichissantes pour le groupe. L'animation des forums exige du modérateur qu'il maîtrise les habiletés usuelles d'animation, mais il doit également savoir les transposer et les adapter au contexte des échanges textuels et en mode asynchrone (Feenberg, 1987; Hotte, 1995 ; Damphousse, 1996, Lundgren-Cayrol, 1996).

Le modérateur de forum mène un ensemble d'interventions et joue des rôles d'animation qui ont déjà été bien décrits. Mason (1993) et Berge (1995) parlent d'interventions d'encadrement cognitif et pédagogique, d'animation sociale, de soutien technique et de gestion de la discussion. Pour Dimock (1987) et St-Arnaud (1989), les rôles joués par l'animateur peuvent être ceux de directeur, de coach, de facilitateur du contenu, de la procédure et du climat et de répartiteur de tâches.

Feenberg (1987), pour sa part, propose trois types d'interventions spécifiques à l'animation des forums : la contextualisation, le contrôle et les métafonctions. Les interventions de contextualisation visent à introduire le thème de la discussion pour la structurer, pour établir les étapes et le plan de travail et pour s'entendre sur le fonctionnement du groupe (procédure à suivre, code d'éthique, etc.). Elles ont lieu surtout au moment de 
l'amorce des échanges. Les interventions de contrôle se veulent le plus souples possible. Le modérateur veille à la cohésion et à la productivité du groupe, au maintien de la participation, à la progression de la tâche, etc. Les interventions que Feenberg qualifie de métafonctions amènent le modérateur à poser un regard global sur l'activité du groupe pour l'aider à dégager le sens de son travail. À l'ouverture d'un forum, elles proposent l'orientation à donner aux travaux et à la clôture, elles proposent au groupe une vision d'ensemble de ce qui a été réalisé. Après la réalisation de chaque étape, elles prennent la forme de résumés ou de conclusions afin d'établir des liens entre elles.

Dans le contexte de la formation à distance, c'est le formateur qui joue le rôle de modérateur et qui prend en charge le groupe. Son rôle consiste à favoriser la participation des apprenants en fournissant un soutien à la motivation et un encadrement pédagogique. Comme le modérateur, il exerce également des fonctions d'hôte social, d'animateur des échanges et de gestionnaire du travail du groupe. Dans les écrits, on explique que les fonctions d'animation sont multidimensionnelles. Elles portent sur plusieurs niveaux du discours : pédagogique, cognitif, métacognitif, organisationnel et social. On souligne également qu'elles ont des effets sur la cohésion et la productivité du groupe (Winn, 1990; Tennyson, Elmore et Snyder, 1990 ; Brown et Palincsar, 1987; Lundgren-Cayrol, 1996).

Pour certaines activités menées dans le cadre de forums électroniques, le formateur partagera ses responsabilités de modérateur avec les apprenants puisqu'il doit favoriser et encourager le développement de leur autonomie. À tour de rôle, les apprenants se verront confier une partie ou la totalité des fonctions d'animation. La pratique d'auto-animation est un moyen de développer sa responsabilité par rapport à l'apprentissage puisqu'il doit être actif et s'employer, comme le formateur, à clarifier les contenus, à établir des liens et des associations entre les idées, à formuler des hypothèses, à résoudre des problèmes et à évaluer les résultats (voir tableau 2.3). 
Tableau 2.3

\section{PARTAGe DU RÔLE DE MOdÉRATEUR ENTRE LE FORMATEUR ET LES APPRENANTS}

\begin{tabular}{|c|c|c|}
\hline \multirow{2}{*}{$\begin{array}{l}\text { Fonctions d'animation } \\
\text { d'un groupe virtuel }\end{array}$} & \multicolumn{2}{|c|}{ Partage du rôle de modérateur } \\
\hline & $\begin{array}{l}\text { Responsabilités } \\
\text { du formateur }\end{array}$ & $\begin{array}{l}\text { Responsabilités } \\
\text { de l'apprenant }\end{array}$ \\
\hline Contextualisation & $\begin{array}{l}\text { - Souhaiter la bienvenue. } \\
\text { - Proposer un ordre du } \\
\text { jour. } \\
\text { - Expliquer les règles de } \\
\text { la nétiquette. }\end{array}$ & $\begin{array}{l}\text { - Ouvrir la discussion. } \\
\text { - Clarifier le sujet de } \\
\text { discussion. } \\
\text { - Formuler les arguments } \\
\text { de départ. }\end{array}$ \\
\hline $\begin{array}{l}\text { Surpervision / } \\
\text { Monitoring }\end{array}$ & $\begin{array}{l}\text { - Inciter et encourager la } \\
\text { participation régulière. } \\
\text { - Reconnaître le groupe } \\
\text { comme une entité à part } \\
\text { entière pour créer une } \\
\text { synergie entre les } \\
\text { participants. }\end{array}$ & $\begin{array}{l}\text { - Reconnaître et } \\
\text { encourager les } \\
\text { commentaires des pairs. } \\
\text { - Inviter les pairs à une } \\
\text { participation régulière. }\end{array}$ \\
\hline $\begin{array}{l}\text { Maillage de la } \\
\text { discussion }\end{array}$ & $\begin{array}{l}\text { Extraire et mettre en } \\
\text { lumière les grandes } \\
\text { trames de la discussion. } \\
\text { - Clôturer la discussion } \\
\text { par une synthèse. }\end{array}$ & $\begin{array}{l}\text { - Gérer la surcharge } \\
\text { d'information lorsque la } \\
\text { discussion s'embrouille. } \\
\text { - Essayer d'extraire et de } \\
\text { mettre en lumière les } \\
\text { liens entre les idées. } \\
\text { - Essayer de régler les } \\
\text { problèmes de } \\
\text { compréhension de } \\
\text { contenu, de clarté et } \\
\text { de pertinence. } \\
\text { - Participer à la synthèse } \\
\text { de clôture. }\end{array}$ \\
\hline
\end{tabular}

\section{LA COHÉSION ET LA PRODUCTIVITÉ DU GROUPE}

Cohésion et productivité sont deux conditions à réunir pour réaliser une collaboration fructueuse. Comme nous allons le montrer, elles sont étroitement liées. Elles sont empreintes de subjectivité et ne se mesurent qu'à travers les perceptions des collaborateurs. La cohésion est un sentiment extrêmement positif qui règne au sein d'un groupe et elle s'exprime par des comportements solidaires et la participation régulière des membres. La productivité se rapporte au sentiment qu'éprouvent les membres d'un groupe lorsque leurs interactions débouchent sur une 
production qu'ils jugent satisfaisante. St-Arnaud (1989) constate que la cohésion et le sentiment d'être productif sont, pour un groupe, des indicateurs de maturité. Un groupe passe typiquement par des stades de croissance et d'évolution qui l'amènent jusqu'à la maturité lorsque ses membres sont devenus de bons collaborateurs. Ils se connaissent bien, savent interagir et ils ont développé un sentiment d'appartenance.

La cohésion n'existe pas sans qu'un groupe soit véritablement constitué. Elle est faite de solidarité et d'autorégulation. Elle est la résultante des forces qui agissent sur les membres pour les inciter à demeurer ensemble (Festinger, 1957). La cohésion accroît sa force lorsque les membres se sentent attirés par le groupe et qu'ils souhaitent profondément y appartenir; c'est l'attrait du groupe. Le degré de proximité et l'identification des membres au groupe sont également des forces de cohésion. La cohésion sert d'indice de maturité du groupe et, si elle est forte, affecte la productivité de manière positive. Dans un groupe, le degré de cohésion et le niveau de productivité s'interinfluencent dans une corrélation positive (Mullen et Copper, 1994).

La productivité s'observe par l'efficacité et la facilité qu'a le groupe de s'entendre sur une cible commune, d'élaborer un plan de travail, de l'implanter, de le réviser en cours de route et de mener à bien son activité. Il n'existe pas de mesure objective pour évaluer la productivité d'un groupe. Les échelles sont aussi nombreuses que les groupes eux-mêmes. Les résultats qu'on obtient sont toujours subjectifs, car ils traduisent des perceptions de satisfaction au sujet de l'efficacité que le groupe a démontrée pour étudier une question, résoudre le problème, prendre une décision, réaliser une activité, etc.

C'est en favorisant l'interdépendance positive entre les apprenants que le formateur peut stimuler la cohésion et augmenter la productivité. Il pourra en prendre la mesure en analysant la qualité des interactions et le contenu cognitif des messages. Il pourra également sonder le groupe directement en administrant un questionnaire sur leurs perceptions (Mullen et Copper, 1994). 


\section{POUR STRUCTURER L'ESPACE DE COMMUNICATION}

Comme nous l'avons expliqué précédemment, la communication est le cœur de la collaboration et, en conséquence, l'espace de communication occupe une place centrale dans l'environnement d'apprentissage. Pour le concepteur pédagogique, la structuration de cet espace est une étape éminemment importante, car elle sera déterminante dans la réussite d'une collaboration. En raison de sa position stratégique, l'espace de communication doit être soigneusement aménagé en fonction de la tâche d'apprentissage et des activités auxquelles participeront les apprenants. Pour mieux comprendre le fonctionnement de la collaboration, il importe de préciser ici la distinction que nous faisons entre la tâche d'apprentissage réalisée individuellement et les activités de soutien à l'apprentissage qui sont menées en groupe ou en équipe.

Nous avons mentionné dans le premier chapitre qu'un groupe collaboratif existe en fonction d'un but commun et partagé par tous. Dans un contexte de formation, chaque apprenant membre d'un groupe vise l'atteinte du but par la réalisation individuelle d'une tâche d'apprentissage. Par ailleurs, les apprenants sont invités à mener des activités en groupe ou en équipes qui les aideront dans la réalisation de la tâche individuelle. C'est ainsi que le groupe agit comme ressource et comme soutien à l'apprentissage et qu'il devient le lieu d'entraide et de collaboration. Par exemple, si la tâche est de produire une analyse des tensions idéologiques de notre société à travers la doctrine économique du néolibéralisme, le concepteur pourra organiser une grande activité de type «débat » sur le bien-fondé de l'approche néolibérale. En s'y préparant par des lectures, par des recherches documentaires et par des discussions en équipes, les apprenants pourront s'approprier le pour et le contre et débattre du sujet de manière convaincante dans le cadre d'un forum de discussion réunissant tout le groupe. Ces activités de recherche, de discussion et de débat permettront aux apprenants de recueillir des informations, de réfléchir à la problématique soulevée et d'analyser les différentes positions. Dans son espace privé, l'apprenant se prépare à mener des activités de groupe ou d'équipe qui, elles, 
se déroulent dans l'espace de communication. Il y présentera de l'information, négociera ses idées avec le groupe et validera les connaissances qu'il a construites. Il s'en retournera par la suite dans son espace individuel pour compléter la réalisation de la tâche d'apprentissage.

L'espace de communication doit donc être aménagé en différents lieux pour:

- discuter en grand groupe,

- coordonner la réalisation des activités collectives,

- réaliser des activités en équipe,

- trouver de l'aide,

- collaborer spontanément,

- socialiser,

- etc.

\section{FORUMS ET LIEUX DE CONSTRUCTION COLLECTIVE DES CONNAISSANCES}

En nous appuyant sur une étude de Ricciardi Rigault et al. (1993), nous proposons d'utiliser les forums électroniques en les aménageant en lieux de:

- télégestion, pour coordonner de la démarche de collaboration;

- télédiscussion;

- télétravail, pour la mise en commun de connaissances et pour l'élaboration collective de connaissances nouvelles;

- télésocialisation, pour canaliser les échanges informels et spontanés ;

- téléassistance, pour offrir conseil et soutien technique.

La figure 2.4 montre comment s'aménagent les lieux d'échange, de travail et d'apprentissage. 
Figure 2.4

\section{L'AMÉNAGEMENT DES LIEUX D'ÉCHANGE, DE TRAVAIL ET D'APPRENTISSAGE}

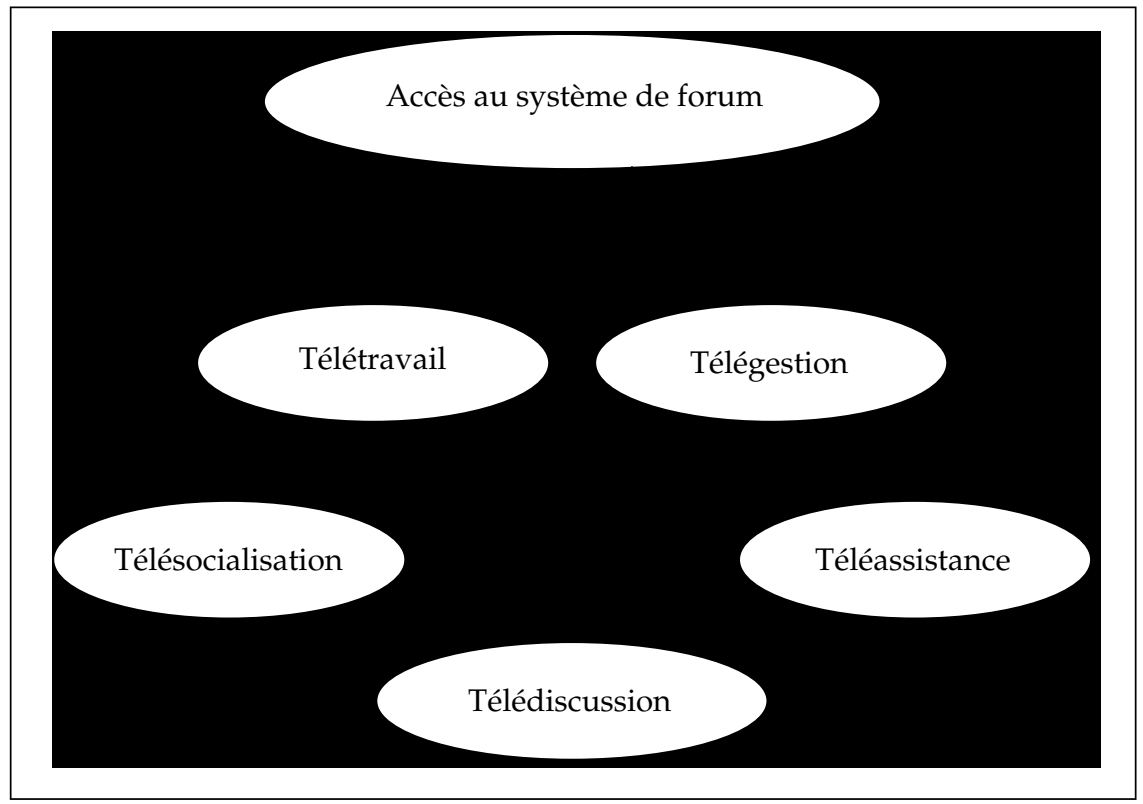

\section{LA TÉLÉGESTION}

Les forums de télégestion sont des lieux de coordination des activités du groupe (voir figure 2.5); ils constituent un centre d'organisation et de gestion pédagogique, cognitive et logistique. Les apprenants s'y retrouvent pour décider d'un plan de travail, pour convenir d'un échéancier, pour traiter de l'organisation temporelle et matérielle des activités, pour former des équipes, pour discuter des orientations cognitives du travail, pour négocier le mode de participation et pour déterminer la contribution et le rôle de chacun. Afin de faciliter le travail de gestion, les apprenants devraient idéalement avoir accès à des outils de planification, de suivi du travail et d'aide à la prise de décision (Kanselaar et Erkens, 1996).

Les lieux de télégestion sont les plus propices pour s'entendre explicitement sur les modalités de la collaboration, pour mettre au clair les contraintes qu'elle impose et les avantages qu'on peut en 
Figure 2.5

LIEUX DE TÉLÉGESTION

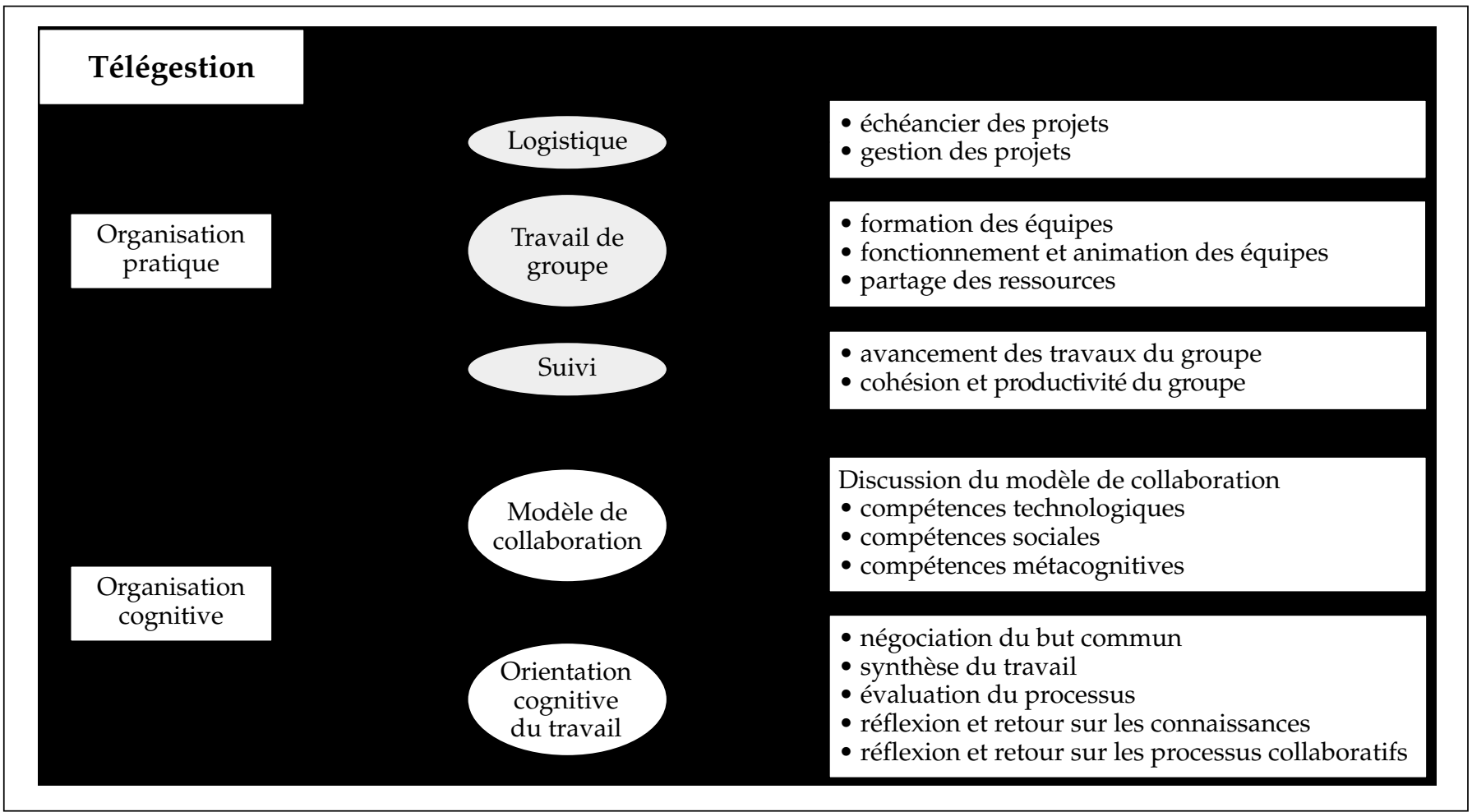


tirer. Les apprenants éveillés aux exigences de collaboration sont invités à y souscrire volontairement et à s'y plier en toute connaissance de cause. Par la même occasion, ils sont amenés à comprendre les buts de l'apprentissage et à donner un sens aux activités collaboratives. Les apprenants qui partagent la même représentation de la collaboration sont plus susceptibles de respecter les modalités de fonctionnement et de réussir leur collaboration.

Les modalités de collaboration et l'orientation cognitive du travail ne sont pas toujours soumises à la discussion par les groupes à qui l'on propose une démarche collective. Surpris par les exigences de la collaboration, les apprenants peuvent manifester de la frustration, de l'insatisfaction et un manque de motivation qui peuvent les amener à abandonner ou être la cause de l'échec de l'activité de formation. Afin d'éviter l'incompréhension des exigences de la collaboration et pour optimiser sa réussite, le groupe doit prendre le temps d'en discuter les conditions et de convenir du modèle de collaboration qui prévaudra.

En plus d'être des lieux de discussion du modèle de collaboration et des aspects pratiques de l'organisation du groupe, les forums de télégestion sont aussi des endroits où le groupe établit un consensus sur l'orientation cognitive à donner au travail et où se confirme le but commun à atteindre.

\section{LA TÉLÉDISCUSSION}

Les forums de télédiscussion sont des lieux privilégiés d'exploration des concepts par la dispute (Ricciardi Rigault et Henri, 1989), d'élaboration et d'évaluation des connaissances par le dialogue et l'interaction (voir figure 2.6). Ils offrent la possibilité de discuter, de poser des questions et de résoudre des problèmes. Ils sont pour les participants la source d'enrichissement cognitif. Hiltz et Turoff (1982) avaient déjà noté la puissance et la synergie au sein des groupes de travail intellectuel qui, par la télédiscussion, produisent des performances supérieures à celles que chaque membre du groupe peut réaliser.

La télédiscussion propose aux apprenants des lieux de construction collective de connaissances en favorisant des échanges approfondis et réfléchis. De manière pratique, les forums de 
Figure 2.6

LIEUX DE LA TÉLÉDISCUSSION

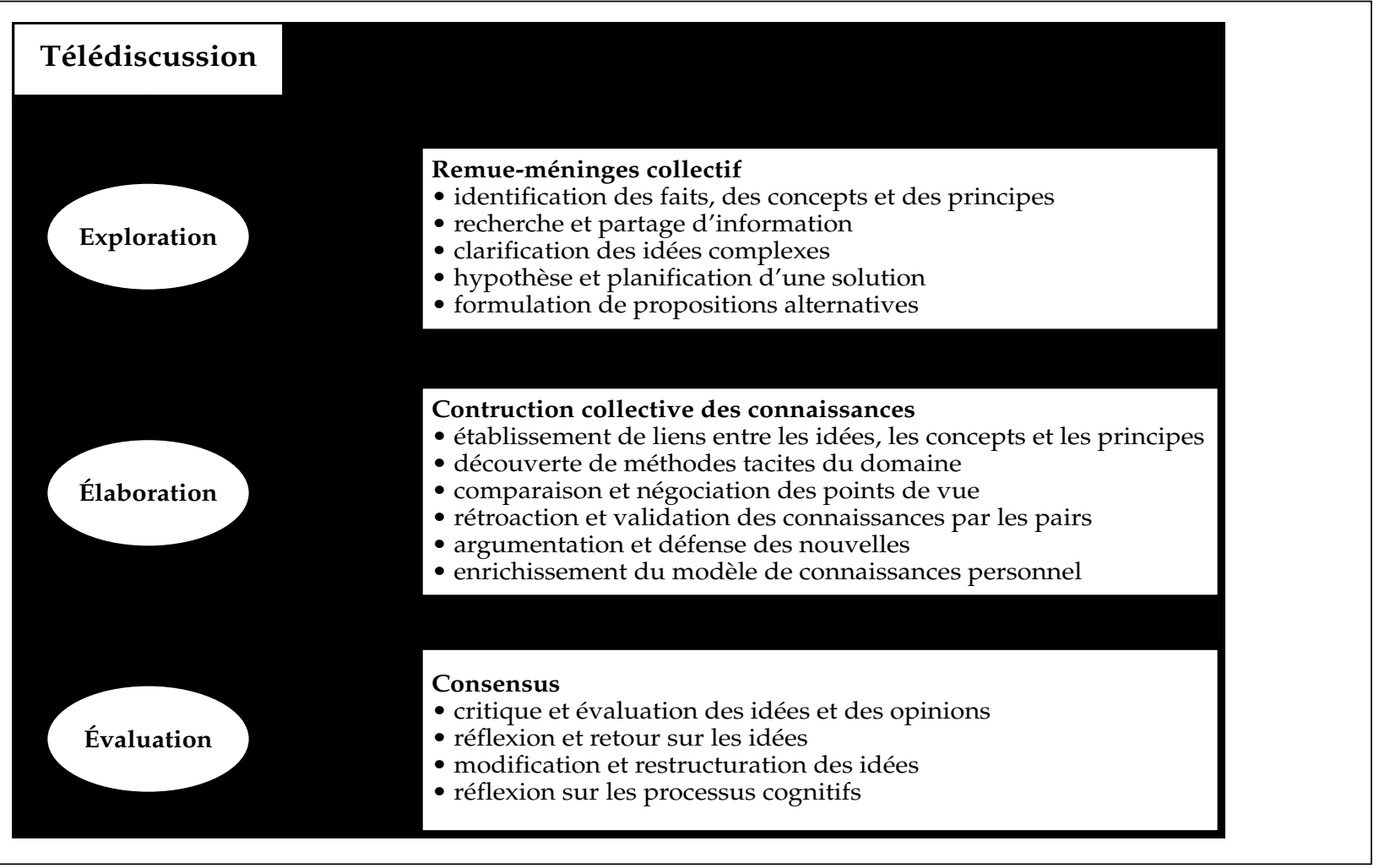


télédiscussion peuvent héberger les échanges de groupes de taille moyenne de plus ou moins 20 apprenants ou de groupes plus nombreux, de 30 à 50 apprenants (Ricciardi-Rigault et al., 1993; Wells, 1995 ; Crotty, 1994 et 1995).

\section{LE TÉLÉTRAVAIL}

Les lieux de télétravail sont réservés aux équipes formées de trois à cinq apprenants pour accomplir des activités ciblées qui précèdent ou qui prolongent le travail fait en privé ou en télédiscussion. Ces travaux peuvent être réalisés à l'intérieur même du forum de télétravail (par exemple: préparation d'une définition, mise en commun d'information sur un sujet donné, analyse de cas, résolution de problème, etc.) ou à l'extérieur de celui-ci (par exemple: constitution d'un dossier, rédaction d'un essai, développement d'un programme informatique, etc.). Dans le premier cas, le forum de télétravail est le lieu qui sert à la planification, à l'organisation et à la réalisation proprement dite de l'activité. Si la réalisation du projet doit se faire à l'extérieur du forum dans l'espace privé, comme ce serait le cas par exemple pour un projet de programmation, les échanges dans le forum de télétravail porteront sur la planification et l'évaluation du projet qui est accompli ailleurs dans l'environnement d'apprentissage (voir figure 2.7).

En outre, selon les activités à réaliser, les forums de télétravail peuvent servir de lieu d'incubation et de planification des conversations ou des discussions qui sont menées dans la télédiscussion. Ils peuvent être utilisés pour fragmenter les grands groupes et ainsi optimiser les échanges en évitant qu'il y ait surcharge ou sous-charge d'informations. En raison du nombre réduit de participants, les forums de télétravail représentent des lieux plus intimes et peu menaçants où les apprenants peuvent exprimer librement leurs idées et leurs opinions avant de les partager dans la télédiscussion. Comme les activités réalisées dans les forums de télétravail sont plus circonscrites et souvent plus concrètes, il est plus facile d'y participer parce que les échanges sont plus directs. Les forums de télétravail peuvent alors servir de lieu d'adaptation pour les apprenants qui n'ont pas l'habitude des forums et de la communication en mode asynchrone. Autant que 
Figure 2.7

LIEUX DE TÉLÉTRAVAIL

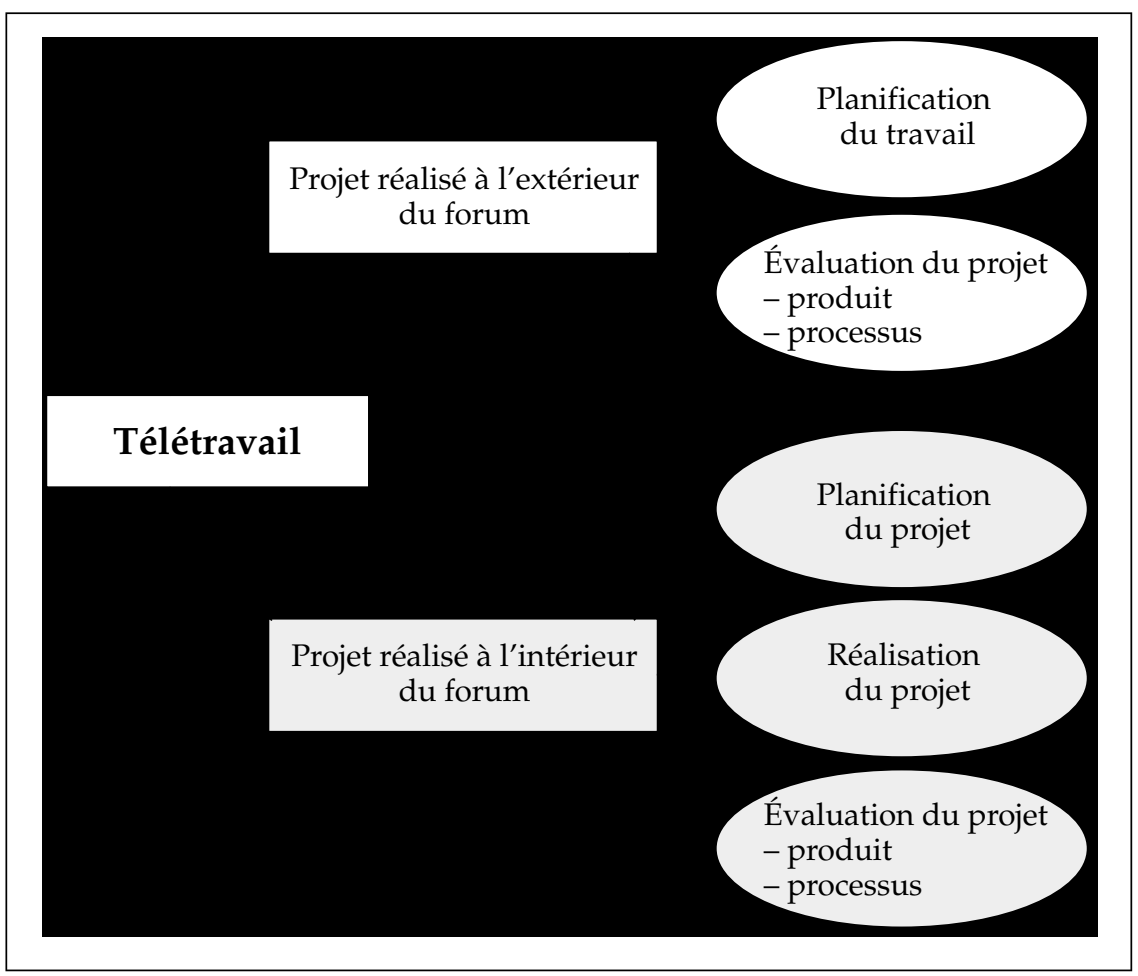

possible, l'animation de ces forums doit être laissée aux apprenants qui, en apprenant à s'auto-animer, développeront leur leadership et leur autonomie.

\section{LA TÉLÉSOCIALISATION}

Dans un environnement virtuel d'apprentissage, on doit tenir compte d'un ensemble de besoins qui ont un impact sur l'apprentissage: besoins cognitifs mais aussi affectifs et psychosociaux. Parmi ceux-ci, la socialisation et le besoin de communiquer de manière informelle ne peuvent être ignorés. Ce sont des besoins universels qui ne manquent pas de s'exprimer au sein des communautés virtuelles. Dans les environnements où l'on n'a pas prévu de lieu ni de moyen pour satisfaire le besoin de 
socialisation, les participants tentent toujours de le combler, avec parfois pour résultat le chaos. Ils peuvent détourner la vocation originale d'un forum en le squattant pour s'en faire un lieu d'échanges informels. Il nous a été donné d'observer à plusieurs reprises des forums qui avaient perdu leur intérêt et leur crédibilité parce que des participants, cherchant à socialiser, émaillaient les échanges de propos qui n'avaient aucun lien avec l'objet de discussion.

Les forums de télésocialisation ont donc comme fonction de canaliser les échanges informels et spontanés qui n'ont pas leur place dans les forums de télégestion, de télétravail ou de télédiscussion. Ils favorisent la création d'un esprit de groupe et le développement de l'appartenance. Les interventions d'un modérateur ont toujours leur place dans ce type de forum. Sans véritablement encadrer la discussion, il veille au ton et au climat des échanges et se soucie que des bons rapports s'établissent entre les participants. Il fait en sorte que le forum de télésocialisation stimule la collaboration. Au besoin, il pourra intervenir avec autorité pour imposer le respect des autres et condamner tout manque d'éthique. Dans certains groupes, le modérateur d'un forum de télésocialisation peut, sans contredit, être un apprenant.

Tout comme on réserve, sur les campus traditionnels, des lieux de rencontres informelles, salons, salles de récréation, cantines, lieux de détente, etc., on doit aménager dans l'environnement virtuel des lieux à vocation de socialisation (voir figure 2.8).

\section{LA TÉLÉASSISTANCE}

Les forums de téléassistance (figure 2.9) offrent de l'aide aux apprenants qui éprouvent des difficultés d'ordre technique ou administratif. Les modérateurs de ces forums verront à ce que les demandes exprimées trouvent réponse s'ils ne peuvent y répondre eux-mêmes. À l'instar des précédents, ils ont comme fonction d'accueillir les propos qui n'ont pas leur place dans les forums de télégestion, de télétravail ou de télédiscussion. 
Figure 2.8

\section{LIEUX DE TÉLÉSOCIALISATION}

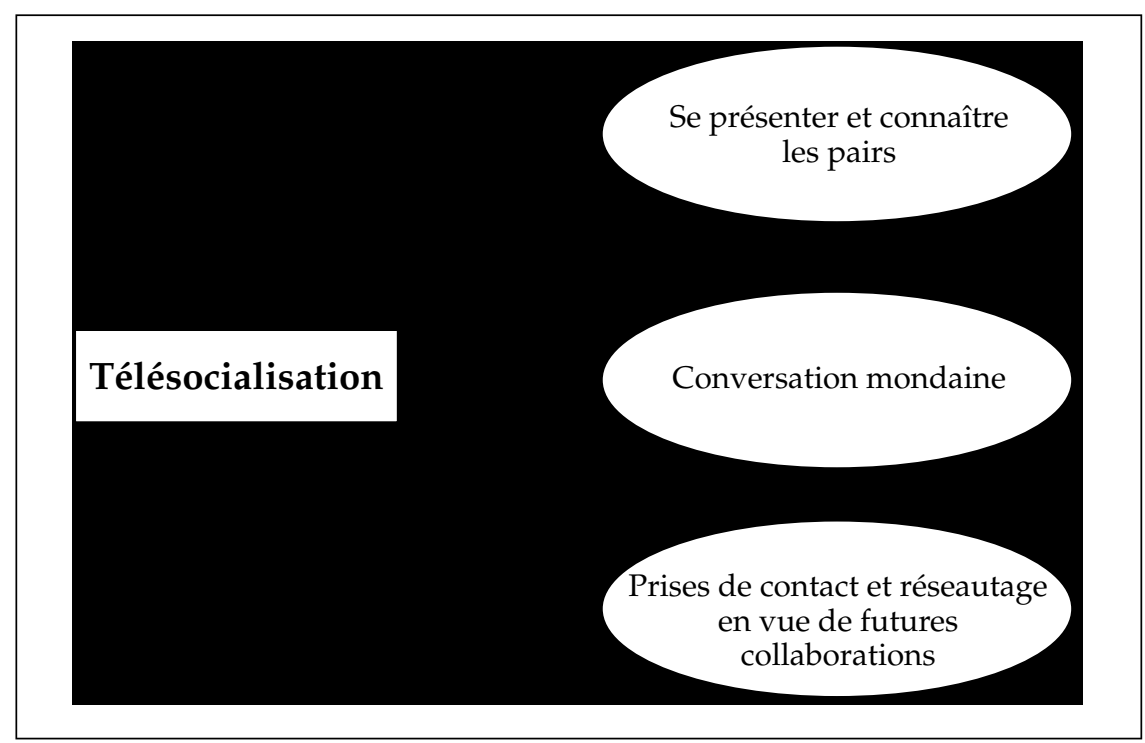

Figure 2.9

LIEUX DE TÉLÉASSISTANCE

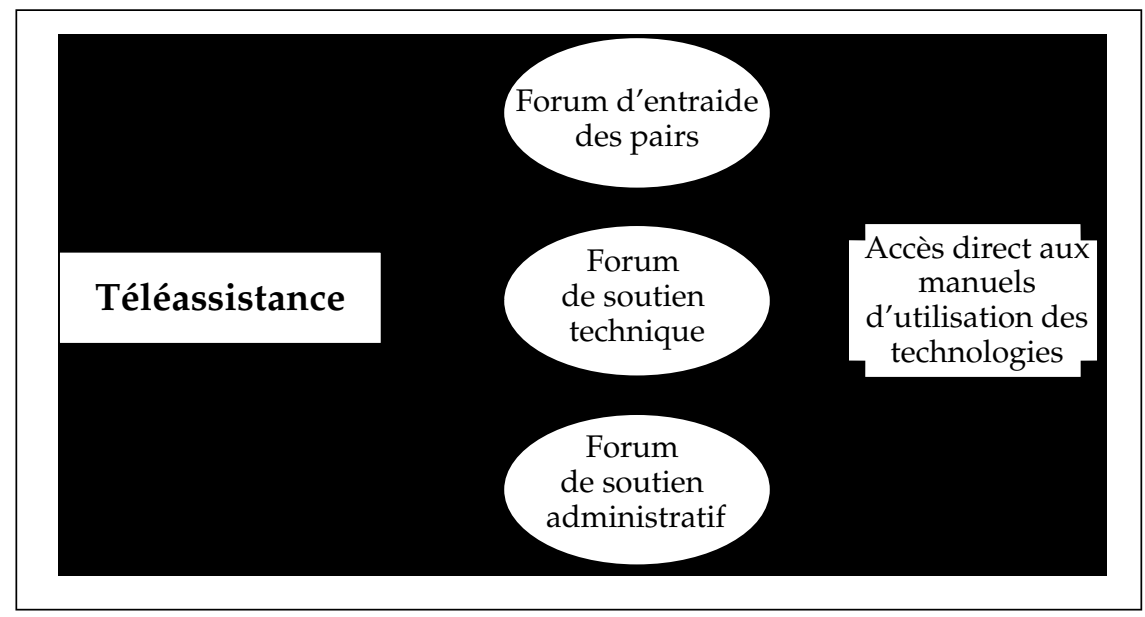




\section{POUR UNE STRUCTURE SOUPLE}

La structure de l'espace de communication que nous venons de décrire servira de repère pour le concepteur d'environnements d'apprentissage. Elle propose des lieux génériques qu'il aménagera et organisera en fonction de la tâche d'apprentissage et des activités de collaboration qu'il proposera au groupe. Le concepteur aura le souci d'une organisation conviviale et ergonomique. L'expérience a montré que les forums de discussions ne doivent pas être trop nombreux, car cela risque de désorienter l'apprenant qui pourra se perdre dans les dédales des forums, ne sachant pas où aller déposer son message, ou encore de le décourager en lui demandant de fréquenter plusieurs lieux et de s'investir dans de trop nombreuses discussions. Combien de forums, sur quels thèmes, pour participer à quelles activités, en combien de temps ? Les réponses viendront d'une démarche d'ingénierie qui s'appuie sur l'analyse du contenu et de la structure de la tâche d'apprentissage et des activités retenues.

Soucieux de demeurer ouvert aux besoins des apprenants, le concepteur aura prévu pour le formateur et même pour les apprenants la possibilité de créer de nouveaux lieux en cours de formation. Il aura laissé le champ libre à la création de lieux émergents pour accueillir des collaborations spontanées, plus ou moins formelles, pour répondre à un besoin et pour soutenir la démarche de collaboration. 


\section{Chapitre 3}

\section{Modèle et ressources de collaboration à distance}

ans le premier chapitre, nous avons montré que la collaboration se distingue de la coopération en cela qu'elle exige de l'apprenant une plus grande autonomie pour l'exercice d'un plus grand contrôle sur son apprentissage. Nous avons aussi souligné que les deux démarches se rejoignent en ayant comme valeur fondamentale l'engagement envers le groupe. Dans le deuxième chapitre, nous avons expliqué qu'entre apprenants autonomes et capables d'autogestion la collaboration se réalise à travers la conversation et s'impose comme modèle de communication. Nous avons vu par la suite que dans les environnements de collaboration l'espace de communication devient central, s'organisant en lieux de conversation de télégestion, de télédiscussion, de télétravail, de télésocialisation et de téléassistance. Les apprenants y interagissent par écrit dans le cadre de forums électroniques. Le succès de ces échanges textuels, avec les forces et les limites qu'on leur reconnaît, repose entre autres sur la participation volontaire et bienveillante des apprenants, sur des modalités de communication acceptées de tous, sur la conciliation des besoins personnels des apprenants avec le but que le groupe s'est 
donné, sur la motivation qui doit être authentique, sur le sentiment d'une profonde satisfaction à appartenir au groupe et sur la plus-value qu'apportent le groupe et le travail en groupe. Quant à l'animation, elle fait également partie des facteurs de succès des groupes virtuels. Sous la responsabilité principale du formateur, l'animation s'appuie sur les principes connus de l'animation des groupes présentiels auxquels s'ajoute le savoir-faire spécifique à l'animation des forums électroniques.

Dans ce troisième chapitre, nous présentons la base d'un modèle de collaboration entre apprenants et nous traitons des ressources de collaboration que sont le groupe et les outils technologiques présents dans l'environnement d'apprentissage. Le chapitre est divisé en trois parties qui correspondent aux trois composantes de base du modèle de collaboration proposé: la communication d'idées au sein des groupes, l'engagement des apprenants et la coordination de leurs activités. Nous verrons alors comment le groupe devient ressource d'apprentissage et les outils technologiques qui peuvent soutenir le processus d'apprentissage et de collaboration. Les outils que nous décrirons viennent se greffer à l'espace de communication et sont exploités en concomitance avec les forums électroniques.

\section{LE GROUPE ET LA COLLABORATION}

L'étude de la collaboration conduit inévitablement à s'intéresser au groupe, à ses caractéristiques et à son fonctionnement. C'est pourquoi il est essentiel de savoir ce qu'on entend par groupe afin de pouvoir jeter les bases d'un modèle de collaboration et $\mathrm{d}^{\prime}$ apprentissage en groupe.

\section{QU'EST-CE QU'UN GROUPE?}

Le groupe n'est pas un concept flou. Il est défini par les recherches en psychosociologie comme une entité collective complexe et dynamique dont l'activité est régie par des principes de fonctionnement. Un groupe n'est pas un simple regroupement d'individus puisque d'autres conditions doivent être réunies pour que des personnes constituent un groupe. Pour définir ce qu'est un 
groupe, nous puisons chez plusieurs auteurs ${ }^{1}$ l'explication de sa raison d'être et quelques principes qui nous font comprendre son fonctionnement.

Un groupe se définit principalement par sa taille, par la relation qui s'établit entre les individus et par le but qui les rassemble. Les petits groupes, dont le nombre n'excède pas 12, et les grands groupes, qui peuvent réunir une trentaine de personnes et même un peu plus, ne fonctionnent pas de la même manière. Pour être efficaces, les grands groupes sont parfois divisés en sous-groupes. On veut ainsi rendre la communication plus facile, encourager le partage, favoriser le développement de solides relations et stimuler l'interdépendance. Mais, on le sait, il ne suffit pas de mettre des personnes en présence les unes des autres pour qu'un groupe existe, pour que des relations se tissent et pour qu'une vie collective s'organise (St-Arnaud, 1989).

\section{Un groupe est plus $q u^{\prime}$ un regroupement}

Groupe et regroupement ne sont pas des notions équivalentes: elles renvoient à deux types de rassemblement qui répondent à des attentes différentes. Comme le rapporte Damphousse (1996), les groupes et les regroupements ont un point en commun: ils partagent un même but qui répond aux intérêts des membres. Ce qui les distingue, c'est l'interaction entre les membres et les relations qui se tissent entre eux. Dans le cas des regroupements, les relations humaines sont généralement superficielles. Les membres manifestent de l'inertie et de la passivité. Ils ne sont pas portés à agir et à engager des actions communes dans la conduite d'actions collectives (p. 24). Ils laissent aux organisateurs, qui sont une petite minorité de membres, le soin de les représenter, de parler et d'agir en leur nom. En revanche, le groupe réunit des personnes qui veulent se mobiliser pour une cause et qui ont une cible commune. Les membres du groupe ont un telle perception de la cible qu'elle justifie et rend nécessaire la participation active de tous les membres aux actions du groupe (p. 24). Cette perception

1. Bales, 1950 ; Shaw, 1981 ; St-Arnaud, 1989 ; Damphousse, 1996 ; Legendre, 1993 ; Festinger, 1950 ; Dimock, 1987 ; Lewin, 1948. 
de la cible commune, particulière au groupe, ne s'impose pas à tous de façon spontanée. À la naissance du groupe, la cible peut être diffuse, mais elle deviendra une réalité concrète et de plus en plus significative grâce aux interactions des membres. Le groupe doit prendre le temps et se donner les moyens pour en construire une représentation commune et pour qu'elle s'installe solidement dans la culture du groupe.

La cible, ou le but commun, est à l'origine du groupe. Partagée et valorisée, elle exprime une intention et une finalité. Au début, lorsqu'il entreprend une collaboration, le groupe n'a pas encore d'objectifs formels et précis. Ceux-ci, contrairement au but qui représente le résultat à atteindre, spécifient les tâches à accomplir. Ils se préciseront graduellement au cours des échanges et des négociations. Ils seront formulés de manière directe, simple, mesurable et réalisable. Les objectifs collectifs, compatibles avec la raison d'être du groupe, sauront aussi satisfaire les objectifs individuels. Chaque membre recherchera donc l'atteinte de ses propres objectifs à travers l'action du groupe.

\section{La cible commune des groupes d'apprentissage}

Les groupes d'apprentissage ont aussi une cible commune qui se présente en deux volets :

- la réussite individuelle de l'activité de formation dans laquelle l'apprenant s'est engagé ;

- l'apprentissage avec les autres (Damphousse, 1996).

Pour qu'un groupe d'apprentissage existe, les apprenants doivent donc avoir, en plus du désir de réussir, la volonté de participer et de collaborer, c'est-à-dire de s'engager dans la réalisation d'activités collectives et accepter le mode de fonctionnement de groupe avec ses gratifications et satisfactions, mais aussi ses exigences et contraintes.

\section{LES BASES D'UN MODÈLE DE COLLABORATION POUR LES GROUPES D'APPRENANTS}

Le groupe est une entité vivante qui naît, croît, atteint une maturité et s'éteint. Ses stades de développement sont connus et abondamment documentés; ils ont d'ailleurs déjà été modélisés par 
St-Arnaud (1989). Il nous est cependant impossible, dans le cadre du présent travail, de rendre compte de manière exhaustive des nombreux travaux se rapportant au groupe et à son fonctionnement. Nous limitons notre étude au processus de collaboration qui se vit au sein d'un groupe. Rappelons que notre but n'est pas de décrire le processus de croissance des groupes d'apprenants, mais :

- de comprendre comment s'articule le travail intellectuel au sein d'un groupe d'apprenants, déjà constitué ou en voie de l'être, qui collabore pour apprendre dans un environnement virtuel ;

- d'identifier les exigences cognitives de ce travail intellectuel ;

- de décrire les outils technologiques qui peuvent leur être utiles.

Nous tenons l'existence du groupe pour un fait acquis et nous tentons de modéliser l'activité cognitive surtout. Nous sommes par ailleurs conscientes qu'on ne peut pas espérer que des apprenants regroupés dans un environnement virtuel actualisent spontanément le potentiel du groupe pour collaborer et apprendre. Il faut pour cela que des méthodes et des moyens soient mis en œuvre pour que le groupe naisse, croisse et atteigne une certaine maturité ${ }^{2}$.

\section{Trois composantes d'un modèle de collaboration}

Les apprenants qui ont choisi de se joindre à un groupe pour apprendre en collaborant ont compris qu'ils doivent participer activement aux activités du groupe; lorsqu'ils cessent de participer, ils cessent de collaborer et le groupe cesse d'exister: la communauté d'apprentissage se dissout. La participation est essentielle à la vie de groupe et constitue la base de la collaboration.

2. L'étude de Damphousse (1996) est fort éloquente à ce sujet. Elle montre que certains concepteurs de cours qui réunissent des étudiants dans des forums électroniques pour les faire collaborer ne prennent aucun moyen pour stimuler la croissance des groupes. Ces concepteurs ne cachent pas leur déception face au désintéressement des étudiants et blâment la technologie sans être conscients que rien n'a été fait pour que les apprenants se donnent un but commun et pour qu'ils comprennent qu'ils sont rassemblés pour apprendre ensemble. 
Comme nous l'avons expliqué dans les chapitres précédents, la démarche d'apprentissage collaboratif reconnaît à l'apprenant et au groupe une certaine liberté. Ils doivent adhérer librement au groupe et vouloir communiquer pour travailler et apprendre ensemble. La collaboration exige en outre l'utilisation de méthodes de gestion pour coordonner l'activité des apprenants tout en respectant leur autonomie. La collaboration est donc faite:

- de communication pour alimenter la réflexion sur l'objet de la collaboration, pour réaliser des tâches et pour tisser des relations ;

- d'engagement pour assurer une contribution cognitive et sociale significative, pour se mettre au service du groupe et pour mobiliser les efforts en vue de la réussite;

- de coordination pour optimiser l'efficacité de l'activité du groupe.

La figure 3.1 représente les trois composantes de base d'un modèle de collaboration.

Figure 3.1

Composantes DE BASE DE LA DYNAMIQUE DE COLLABORATION

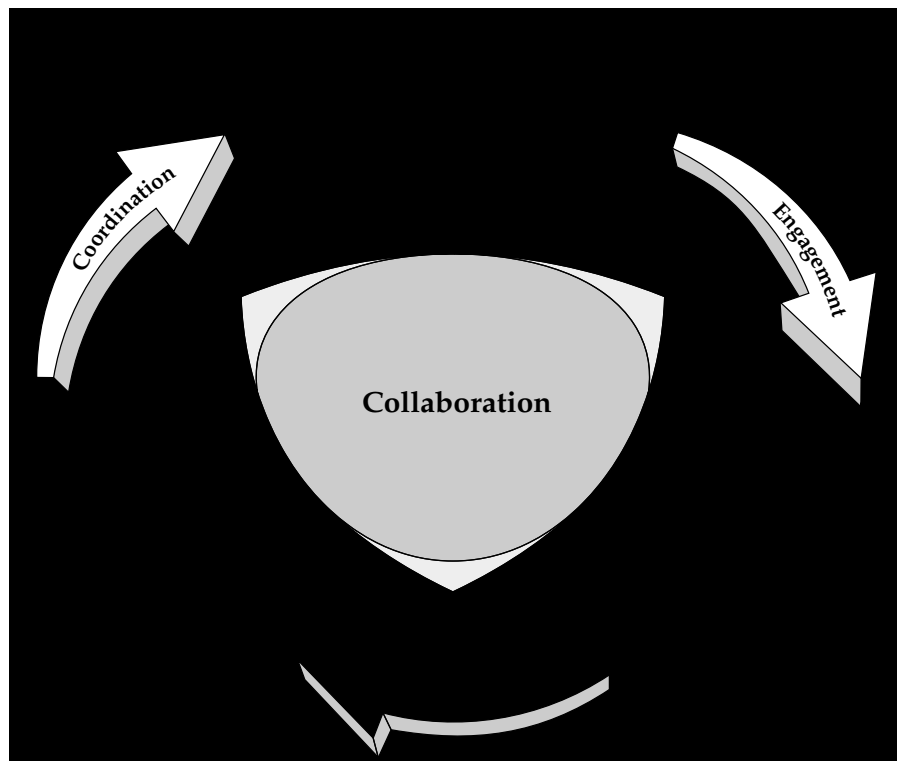


Nous les décrivons dans les pages qui suivent et nous expliquons comment elles agissent dans le cadre de forums électroniques. Nous identifions pour chaque composante les outils technologiques qui peuvent soutenir le travail du groupe. Cette description aidera, espérons-nous, les concepteurs à se représenter les activités et les outils de collaboration d'un environnement d'apprentissage virtuel.

\section{LA COMMUNICATION DES IDÉES}

Dans notre modèle, la communication est abordée sous un angle cognitif et elle se rapporte au travail mis en œuvre par les apprenants pour partager leurs idées et pour construire ensemble leurs connaissances.

Lorsque l'apprenant participe à une démarche collaborative, il s'engage dans un processus de communication cognitive qui l'amène à :

- exprimer des idées afin de les partager avec le groupe ;

- établir des liens entre les idées exprimées (les siennes et celles des autres) afin de faire émerger de nouvelles idées ;

- structurer les idées (les siennes et celles des autres) pour leur donner un sens et pour construire des connaissances.

Ce travail, qui devraient aboutir à la construction de connaissances nouvelles, s'effectue en groupe par la réalisation d'une suite d'activités ou de tâches à caractère cognitif qui se déroulent selon un ordre croissant de complexité. Les apprenants sont invités à "penser ensemble» pour «apprendre ensemble». L'effort cognitif à fournir augmente suivant la progression des échanges. D'une étape à l'autre - expression d'idées, établissement de liens entre les idées et structuration des idées -, la complexité cognitive croît de même que les besoins cognitifs de l'apprenant (tableau 3.1). Nous tentons dans cette partie de cerner les besoins cognitifs au regard de la communication des idées et de décrire les outils technologiques que l'environnement devrait fournir pour y répondre. 
Tableau 3.1

\section{DYNAMIQUE DE LA COMMUNICATION DES IDÉES ET ACTIVITÉS COGNITIVES CORRESPONDANTES}

\begin{tabular}{|l|l|}
\hline $\begin{array}{l}\text { Dynamique de la communication } \\
\text { des idées }\end{array}$ & Exemples d'activités et tâches cognitives \\
\hline Exprimer des idées afin de les partager & $\begin{array}{l}\text { - Participer à un remue-méninges } \\
\text { - Participer à une discussion } \\
\text { - Transmettre des informations } \\
\text { - Développer les idées qui ont été } \\
\text { présentées par d'autres } \\
\text { - Questionner et se questionner } \\
\text { - Commenter, critiquer et évaluer } \\
\text { - Prendre position }\end{array}$ \\
\hline $\begin{array}{l}\text { Établir des liens entre les idées pour faire } \\
\text { émerger des idées nouvelles }\end{array}$ & $\begin{array}{l}\text { - Analyser des énoncés pour les épurer } \\
\text { et en extraire l'essentiel } \\
\text { - Réfléchir, induire, déduire } \\
\text { - Établir des liens entre les concepts, } \\
\text { les principes, les stratégies } \\
\text { - Comparer les différents points de vue } \\
\text { et les idées divergentes }\end{array}$ \\
& $\begin{array}{l}\text { - Cerner les champs théorique et } \\
\text { applicatif et en trouver les assises }\end{array}$ \\
\hline $\begin{array}{l}\text { Structurer les idées pour leur donner } \\
\text { un sens }\end{array}$ & $\begin{array}{l}\text { - Organiser les idées } \\
\text { - Formaliser les idées } \\
\text { - Élaborer une carte conceptuelle } \\
\text { - Développer un modèle de } \\
\text { connaissances }\end{array}$ \\
\hline
\end{tabular}

\section{EXPRIMER DES IDÉES AFIN DE LES PARTAGER}

Dans une démarche d'apprentissage collaboratif, les apprenants mettent d'abord en branle un processus d'exploration exigeant des habiletés cognitives et métacognitives de haut niveau. Ils commencent cette exploration de l'objet à connaître en exprimant de manière cohérente, significative et compréhensible pour les autres, les idées qui leur viennent à l'esprit sur cet objet. Puis, ils s'interrogent sur leurs propres idées, expriment ce qu'ils pensent et réagissent aux propos et commentaires de leurs pairs. Ils font des recherches pour repérer les limites et la structure du domaine étudié et se prononcent sur le contenu de leurs lectures et sur la pensée des auteurs consultés. Ce travail peut faire surgir des conflits cognitifs qu'ils doivent s'employer à résoudre. 
Pour que l'exploration soit réalisable et efficace, les apprenants doivent pouvoir trouver dans l'environnement suffisamment d'informations pertinentes sur l'objet d'étude. Par ailleurs, le formateur leur fournira des indices et leur proposera des pistes de réflexion qui les amèneront à explorer les diverses facettes de l'objet d'étude. En somme, pour stimuler l'expression des idées, les apprenants ont surtout besoin d'information et du soutien d'une personne compétente pour orienter leur recherche et animer leurs échanges.

\section{Ressources de l'environnement technologique}

La principale ressource technologique de soutien à l'expression des idées est sans contredit le forum électronique. Ce que l'on attend de cet outil, c'est qu'il soit assez souple pour permettre la réalisation d'activités libres, spontanées et non structurées, comme le remue-méninges, pour explorer l'objet à connaître en laissant libre cours à l'expression des idées. Dans ce cas, il n'est pas souhaitable que le logiciel de forum dicte une structure pour la discussion ou qu'il la prenne en charge; il ne pourrait alors qu'imposer des contraintes au discours et limiter la spontanéité de la communication.

Si le logiciel de forum électronique ne doit pas régir la structure des échanges, il doit par ailleurs en permettre une certaine organisation. Pour explorer un thème ou un domaine, on doit pouvoir créer pour chacune des idées fortes ou des problématiques cruciales un forum de discussion spécifique. L'interface du logiciel doit permettre de visualiser la structure de ces forums et d'identifier les forums qui se rapportent à chacun des thèmes. L'apprenant pourra ainsi s'orienter dans l'espace de communication et repérer facilement le bon forum pour y déposer son message.

Les logiciels que nous connaissons sont assez rudimentaires. Pour retrouver les idées exprimées dans un forum, ils offrent des fonctionnalités limitées qui procèdent par tri : tri par thème de discussion, par sujet du message, par date d'envoi, par auteur et par type de document. Ils sont en revanche assez conviviaux, faciles à apprendre, faciles à utiliser et leurs interfaces permettent la manipulation intuitive des commandes. Un logiciel qui n'aurait 
pas ces fonctions de base pour le repérage des idées et une convivialité minimale ne saurait être retenu surtout lorsque les apprenants en sont à leurs premières expériences de communication télématique.

\section{ÉTABLIR DES LIENS ENTRE LES IDÉES POUR FAIRE ÉMERGER DES IDÉES NOUVELLES}

Par l'exploration collective d'un thème ou d'un domaine, les apprenants sont amenés à faire un traitement de reconstruction de l'information par l'analyse, la comparaison, l'induction et la déduction. Ils établissent des liens entre les idées et élaborent des inférences qui conduisent au développement de microstructures (Deschênes, 1995). Ces microstructures, qu'ils bâtissent progressivement par la mise en relation des idées, mènent à la formation de nouveaux concepts et donnent naissance à une première perception du domaine et de ses limites.

Pour mettre en relation les idées exprimées par les uns et les autres et pour les organiser dans une structure, les apprenants travaillent sur un ensemble souvent volumineux de messages où sont exprimés les points de vue de leurs pairs. Ils sont alors confrontés à deux problèmes: celui du volume de l'information à traiter et de sa complexité.

\section{Ressources de l'environnement technologique}

Pour traiter efficacement l'information contenu dans les forums électroniques, pour établir des liens entre les idées et pour stimuler l'émergence de concepts nouveaux, les apprenants doivent pouvoir consulter rapidement et facilement les messages et disposer d'un aperçu complet, mais résumé ou condensé, de ce qui a été dit.

Ils devraient retrouver dans l'environnement des fonctionnalités permettant:

- l'accès et le repérage de l'information pour recenser les idées contenues dans la banque de messages;

- le tri et le classement des messages pour regrouper les idées ; 
- l'annotation pour se donner des repères ;

- la création de liens entre les idées.

Insistons d'abord sur le fait que ces fonctionnalités devraient être accessibles hors ligne, sur le poste de travail de l'apprenant afin qu'il puisse travailler en toute quiétude sur la banque de messages. Les fonctions d'accès et de repérage de l'information devraient permettre

- une lecture rapide des messages,

- l'accès à des condensés ou à des résumés des interventions,

- la recherche par des fouilles dans le texte ${ }^{3}$.

Une fois l'information repérée, l'apprenant devrait pouvoir faire un premier regroupement d'idées avec un outil de tri puis utiliser des outils d'annotation pour consigner ses observations et ses commentaires sur les liens (typés ou non) entre les idées. Ces notes pourront être utilisées par la suite pour développer des idées nouvelles, pour rédiger d'autres messages à déposer dans les forums ou pour produire des documents qui seront déposés dans des espaces communs. Des outils de type hypertexte devraient également pouvoir être utilisés pour créer les liens entre les segments de messages que l'on veut associer et pour obtenir des représentations sous la forme de réseaux et de cartes sémantiques qui pourront être partagées avec le groupe (Klemm et Snell, 1996; Hoadley et Hsi, 1993 ; Guzdial et al., 1995).

\section{STRUCTURER LES IDÉES POUR LEUR DONNER UN SENS}

La structuration des idées permet de construire des ensembles notionnels complets et cohérents à partir des microstructures élaborées par les apprenants. Elle consiste à élaborer des macrostructures (Deschênes, 1995) qui peuvent être organisées selon différents modèles : logique, argumentatif, descriptif, narratif, etc. Le modèle retenu correspondra à l'objet, au but et au contexte de l'apprentissage.

3. On pense à des outils tel que Nomino développé au Centre de recherche LICEF. 
Tableau 3.2

JEU DE RÔLES POUR LA PRODUCTION D'UNE CARTE CONCEPTUELLE: STRATÉGIE POUR LA STRUCTURATION DES IDÉES

\begin{tabular}{|l|l|}
\hline $\begin{array}{l}\text { Élément de la } \\
\text { stratégie }\end{array}$ & \multicolumn{1}{c|}{ Description } \\
\hline Production du groupe & $\begin{array}{l}\text { Une carte conceptuelle organisée selon une structure } \\
\text { logique, argumentative, narrative, descriptive ou autre. }\end{array}$ \\
\hline Matériel de soutien & $\begin{array}{l}\text { - Guide de production d'une carte conceptuelle incluant: } \\
- \text { les règles de construction de différents modèles } \\
\text { de cartes } \\
\text { - les méthodes de travail individuel et collectif } \\
\text { - Outils logiciels utilisés pour la production } \\
\text { - Guide et consignes pour le travail d'équipe }\end{array}$ \\
\hline Distribution des rôles & $\begin{array}{l}\text { Les rôles visent à clarifier les idées exprimées, à les } \\
\text { structurer et à les partager avec le groupe. } \\
\text { - Coordonnateur: il s'assure que chaque membre accomplit } \\
\text { ses tâches à temps; il commente les documents remis } \\
\text { par les membres du groupe; il voit à ce que le travail } \\
\text { corresponde au but que le groupe ou l'équipe s'est } \\
\text { donné. } \\
\text { - Éditeur de la liste de concepts : il consolide et édite les } \\
\text { listes des concepts et des principes produites par chaque } \\
\text { membre; il produit une liste synthèse et la partage avec } \\
\text { le groupe. } \\
\text { - Chercheur d'information : il enrichit la liste synthèse en } \\
\text { aidant les éditeurs à trouver l'information manquante. } \\
\text { - Éditeur de la carte conceptuelle: à partir de la liste } \\
\text { synthèse, il construit une carte conceptuelle montrant les } \\
\text { liens et la structure; il la soumet au groupe pour } \\
\text { discussion, amélioration et validation. }\end{array}$ \\
\hline
\end{tabular}

En manipulant ainsi les idées, l'apprenant s'approprie l'objet d'étude; il le reconceptualise et en développe une compréhension plus approfondie. Cette démarche individuelle est suivie d'une mise en commun. À son tour, le groupe s'approprie l'objet d'étude de manière originale par la discussion, la négociation et le consensus. Le résultat de ce travail peut prendre plusieurs formes: des énoncés de principes, une prise de position commune, des définitions, un texte théorique, un hypertexte, un réseau sémantique, un carte conceptuelle, etc. Pour arriver à réaliser de telles productions, les apprenants ont besoin d'un encadrement qui sera plus ou moins structuré selon leur maîtrise du domaine, leur autonomie et leur connaissance des méthodes de travail de groupe. 
Le recours au jeu de rôles pour l'élaboration d'une carte conceptuelle est un exemple de stratégie d'encadrement ou de guidage qui peut être appliquée pour soutenir le processus de structuration des idées. Nous résumons dans le tableau 3.2 les éléments d'un jeu de rôles imaginé par Klemm et Snell (1996) pour amener un groupe d'apprenants à produire une carte conceptuelle de l'objet d'apprentissage.

\section{Ressources de l'environnement technologique}

Les apprenants doivent pouvoir accéder hors ligne à des dispositifs pour construire des cartes, des plans ou des gabarits qui permettent de visualiser, dans une perspective de plus haut niveau, la macrostructure tout en indiquant les microstructures, c'est-à-dire les liens qui ont été établis entre les idées à l'intérieur des banques de messages. Les outils qui se prêtent à l'élaboration de ces structures sont les outils de modélisation des connaissances, les outils de type hypertexte, les cartes conceptuelles et les outils d'assistance à l'élaboration d'un plan. Ils doivent pouvoir traiter l'ensemble des messages de manière transparente et être utilisés facilement.

Outils de modélisation des connaissances. Il existe des outils spécialisés de structuration des connaissances qui déterminent et contrôlent la structure de l'objet que l'on veut représenter ou modéliser. Ces outils peuvent prendre en charge les idées, les associer et les organiser selon des règles précises. Ils permettent d'identifier le type de lien entre les idées, les principes, les procédures et les faits qui composent le modèle de connaissances: liens de composition, de régulation, de précédence, etc., comme dans l'exemple qui suit ${ }^{4}$. Ce sont de véritables outils d'assistance à la pensée humaine (voir figure 3.2).

4. Le logiciel MOT (Modélisation par Objet Typé) produit au Centre de recherche LICEF est un exemple d'outil de structuration et d'organisation des connaissances. Il est couramment utilisé dans les cours dispensés par la Téléuniversité tant par les concepteurs que par les apprenants. 
Figure 3.2

EXEMPLE DE MODĖLE DE CONNAISSANCES

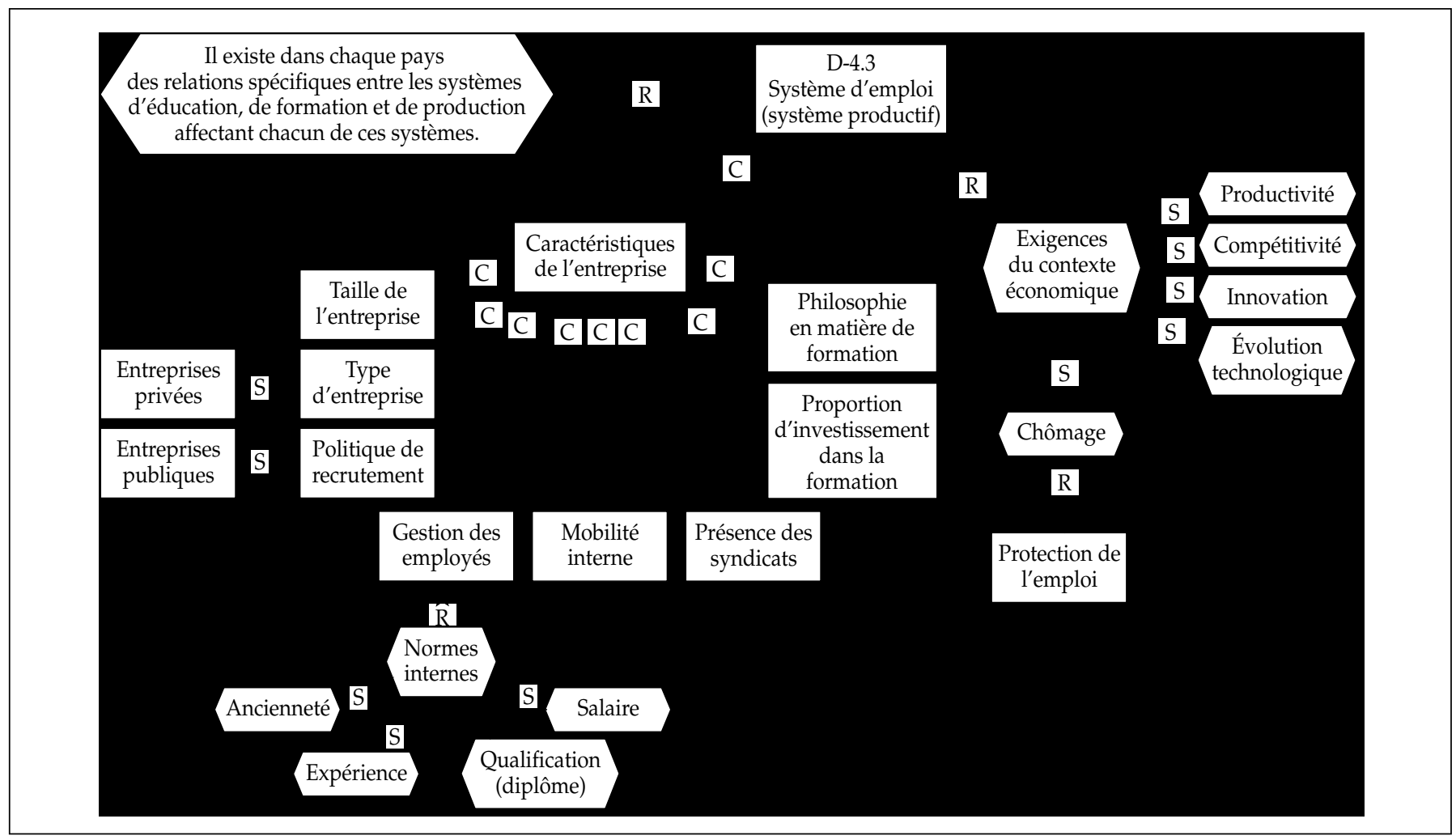


Outils de type hypertexte. L'hypertexte propose deux moyens de concevoir des structures: la collection et la structuration de l'information. Paquin (1993) explique que la collection "permet de regrouper un ensemble d'unités d'information afin de créer des agrégats ayant des propriétés en tant qu'agrégats ». La structuration de l'information regroupe «l'ensemble des opérations qui permettent la manipulation de la structure des documents et des vues multiples sur ces structures ». Sans avoir été prévus pour offrir une assistance à qui veut travailler à la structuration d'un objet de connaissances, ces mécanismes présents dans les systèmes hypertextes peuvent aider l'apprenant dans son processus de structuration des idées.

L'hypertexte ne cesse d'évoluer en raison des nombreuses recherches réalisées par les concepteurs d'interfaces pour l'Internet. On devrait suivre de très près ces développements et envisager de greffer les outils hypertextes aux forums électroniques. Cette intégration devrait permettre de considérer chaque message ou ensemble de messages comme un nœud adressable et de naviguer facilement d'un point à l'autre ${ }^{5}$.

L'outil hypertexte devrait faciliter:

- la consultation du réseau entier des banques de messages, en utilisant des liens pour atteindre et consulter l'information de ces nœuds ;

- la conception de structures, en établissant de nouveaux liens entre ces nœuds;

- la conception de documents emmagasinés sous la forme de nœuds et liés aux messages qui s'y rapportent.

La figure 3.3 schématise l'allure d'une autre carte conceptuelle et de liens hypertextes qui auraient été produits à l'issue d'un forum de discussion.

Cartes conceptuelles. Il existe des outils spécialisés pour construire des cartes conceptuelles; ils permettent d'organiser des informations linéaires en une représentation spatiale indiquant

5. Par exemple, l'outil Astra permet d'extraire et de représenter la structure d'un site Web. Pour les forums basés sur des pages Web, on peut imaginer un outil de ce genre pour dégager des îlots de messages parents et pour en représenter la structure. 
Figure 3.3

\section{UN EXEMPLE DE CARTE CONCEPTUELLE ET DE LIENS HYPERTEXTES}

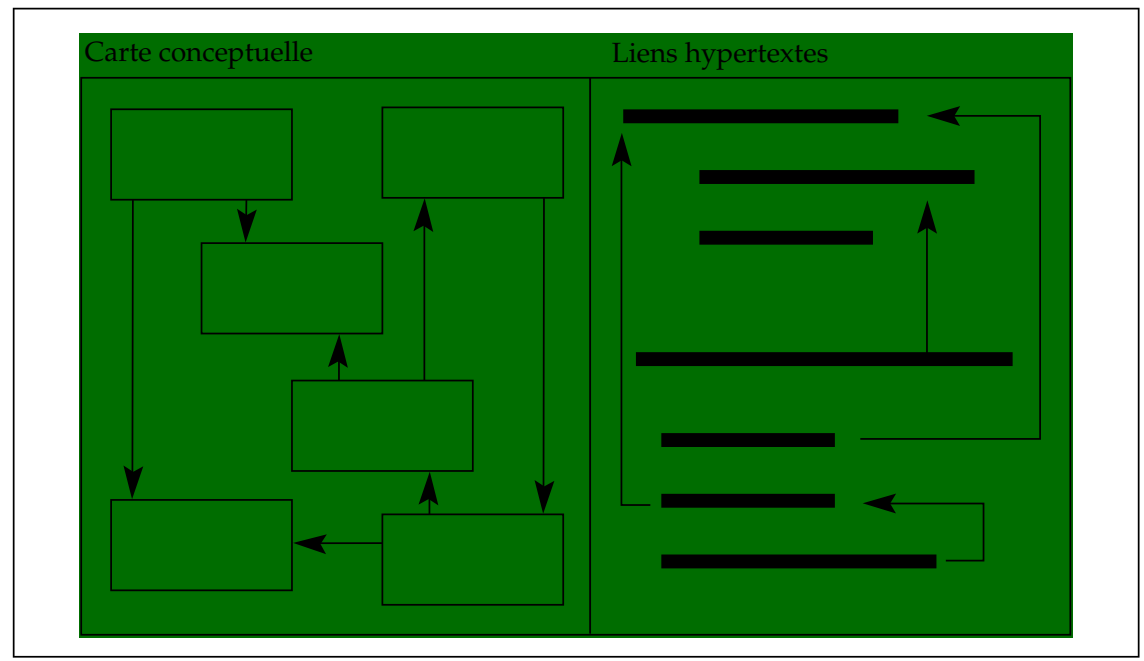

Source : Exemple tiré de Klemm et Snell (1996) et produit avec le logiciel Forum dans un écran à multifenêtrage.

les liens et les relations entre des idées ou des concepts. Ils proposent une organisation hiérarchique en plaçant les concepts plus généraux vers le haut de la carte et les concepts plus spécifiques, vers le bas. Le liens sont nommés pour unir les unités sémantiques. Ces cartes sémantiques sont d'abord et avant tout utilisées comme des outils de négociation de sens (Schroeder et Kenny, 1995) à la phase d'élaboration des connaissances. Toutefois, elles peuvent aussi servir à la phase initiale d'exploration pour colliger des idées et pour commencer à définir des liens et cerner les limites du sujet. Schroeder et Kenny (1995) rapportent que les cartes conceptuelles sont généralement très appréciées par les apprenants et qu'elles profitent surtout à ceux qui les construisent. Ils ajoutent que les cartes préparées par les enseignants ne peuvent aider les apprenants que s'ils ont déjà eu l'occasion d'en concevoir eux-mêmes.

Un outil hypertexte ou un outil de construction de carte conceptuelle peut se révéler une ressource d'apprentissage médiocre si l'apprenant ne sait pas l'exploiter. Les recherches ont 
montré que pour utiliser efficacement un outil hypertexte, 1'usager doit savoir prendre des notes et élaborer un plan. Il semble donc judicieux d'aider les apprenants à développer leurs compétences pour la prise de notes et l'élaboration de plan afin qu'ils puissent tirer profit d'outils hypertextes qui seraient intégrés au logiciel de forum électronique ou offerts en parallèle. Le texte qui suit s'inspire de comptes rendus de recherches menées sur cette question.

Outils d'assistance à la prise de notes. La prise de notes peut avoir trois fonctions: encoder de l'information brute pendant ou après la lecture, l'audition ou le visionnage d'une présentation ; préparer un document qu'on consultera pour se rappeler et mémoriser l'information; reconstruire l'information lorsque les notes sont prises après la lecture, l'audition ou le visionnage (Schroeder et Kenny, 1995).

Les outils de prise de notes sont généralement peu structurés. Ils permettent à l'usager de prendre des notes personnelles, mais aussi de copier et de coller de l'information prise dans un document. Alors que les notes personnelles exigent un effort d'analyse et de synthèse, la fonction "copier - coller » ne demande que le choix de l'information. Pour aider au développement de stratégies de prise de notes personnelles, on choisira un outil qui permet à l'apprenant de revoir l'information notée à partir de différents points de vue (liste des idées fortes, idée forte et les idées $s^{\prime} y$ rapportant, liens qui ont pu être établis entre les idées, etc.) et de la réorganiser en y ajoutant d'autres informations (Röscheisen, Mogensen et Winograd, 1996; Ackerman, 1996 ; Bonk et King, 1995).

Outils d'assistance à l'élaboration d'un plan. Savoir élaborer un plan est une habileté de haut niveau. Il faut être capable d'identifier les relations entre les idées et les concepts et les organiser dans un ordre de subordination. Ce procédé met en évidence les points importants et essentiels d'un contenu, aide à se familiariser avec la structure d'un domaine ou d'un document, à retenir des informations et à les réorganiser dans un ordre significatif. Il serait juducieux de fournir un outil d'assistance à l'élaboration d'un plan qui peut fonctionner sur divers formats de documents (doc, ppt, htm, etc.) et sur des notes personnelles prises sur un document. 


\section{L'ENGAGEMENT ENVERS LE GROUPE}

Nous venons de voir les principaux mécanismes de la communication cognitive, première composante d'un modèle de collaboration, et nous avons brièvement décrit des outils technologiques pour les supporter. Nous abordons maintenant l'étude de l'engagement envers le groupe qui doit animer les collaborateurs.

Nous concevons l'engagement comme une disposition affective et psychologique sans laquelle la collaboration ne peut être envisagée. L'engagement se traduit par la participation active de tous les membres d'un groupe et par d'authentiques efforts pour réaliser les tâches et atteindre le but. La dynamique sociocognitive qui règne au sein d'un groupe peut stimuler, encourager ou encore décourager l'engagement. Des recherches sur l'apprentissage en groupe (Abrami et al., 1995 ; St-Arnaud, 1989 ; Dimock, 1985) ont permis d'identifier trois variables de l'engagement: l'appartenance au groupe, la cohésion du groupe et la perception qu'a le groupe de sa productivité.

\section{L'APPARTENANCE AU GROUPE}

L'appartenance se révèle par les efforts manifestes des apprenants pour s'impliquer dans les travaux du groupe. Le sentiment d'appartenance se développe chez eux lorsqu'ils ont l'impression d'avoir véritablement contribué à l'atteinte du but commun et qu'ils estiment en avoir tiré un bénéfice personnel inestimable (Schrage, 1990). Selon Gutwin, Stark et Greenberg (1995), le sentiment d'appartenance, élément clé du succès de la collaboration, s'installe plus difficilement au sein des groupes virtuels en raison de la pauvreté des canaux de communication qui ne laissent passer que peu d'information contextuelle et peu de renforcements physiques, sociaux ou comportementaux. Le sentiment d'appartenance peut néanmoins être stimulé par quatre prises de conscience:

- l'aspect social de la collaboration; cette prise de conscience veut contrer l'impression d'être seul à travailler devant un ordinateur et sensibiliser aux contraintes du mode de travail en groupe et aux attentes de participation qu'il crée ;

- la nature cognitive de la tâche; cette prise de conscience veut aider l'apprenant à comprendre que ce sont ses ressources 
cognitives qu'il doit mettre à contribution et qu'en canalisant ses efforts dans la même direction que le groupe il participera à la réussite de la tâche ;

- les besoins cognitifs relatifs à la tâche; cette prise de conscience veut aider l'apprenant à gérer et réguler son apprentissage et à apprécier sa progression ;

- l'espace commun et le partage des ressources; cette prise de conscience veut aider l'apprenant à saisir qu'il partage avec ses pairs un univers mental et qu'il se doit de les soutenir vers l'atteinte du but commun en les faisant bénéficier de ses ressources.

Le formateur peut encourager ces prises de conscience en posant des questions comme celles présentées au tableau 3.3 et en utilisant diverses stratégies que nous résumons dans le tableau 3.4.

Tableau 3.3

PRISES DE CONSCIENCE ASSOCIÉES

À L'APPRENTISSAGE COLLABORATIF

Conscience du groupe comme milieu social

Que puis-je attendre des autres membres du groupe?

Comment vais-je interagir avec ce groupe?

Quel rôle vais-je adopter dans le groupe?

Quel rôle les autres membres de l'équipe vont-ils assumer?

Conscience de la nature cognitive de la tâche et de ses exigences

Que sais-je sur le sujet et sur la structure de la tâche?

Que savent les autres sur le sujet et sur la tâche?

Quelles sont les étapes que nous devrons compléter pour accomplir la tâche?

Comment les résultats seront-ils évalués?

Quels sont les outils et le matériel nécessaires pour compléter la tâche?

Combien de temps le travail requiert-il ? De combien de temps disposons-nous?

Conscience des besoins cognitifs relatifs à la tâche

Comment cette tâche s'intègre-t-elle dans ce que je sais déjà?

Qu'ai-je besoin d'apprendre en rapport avec la matière?

De quelle information ai-je besoin?

Ai-je besoin de réviser ma perception et mes idées à la lumière des nouvelles informations?

En m'appuyant sur mes connaissances, puis-je entrevoir les résultats de la tâche?

Conscience de l'espace commun et du partage des ressources

Que doivent faire les autres membres du groupe pour compléter la tâche?

Que font-ils? À quoi travaillent-ils actuellement?

Qu'est-ce qu'ils ont déjà réalisé?

Que feront-ils ensuite?

Comment puis-je aider d'autres étudiants en rapport avec la tâche? 


\section{Tableau 3.4}

\section{INTERVENTIONS POUR STIMULER LE SENTIMENT D'APPARTENANCE}

\begin{tabular}{|c|c|}
\hline Prises de conscience & Interventions pédagogiques \\
\hline $\begin{array}{l}\text { Aspect social de la } \\
\text { collaboration et mode } \\
\text { de travail } \\
\text { Les apprenants } \\
\text { forment un groupe, ils } \\
\text { partagent un but } \\
\text { commun. } \\
\text { Ils travaillent ensemble } \\
\text { et peuvent compter sur } \\
\text { le soutien du groupe. }\end{array}$ & $\begin{array}{l}\text { - Inviter les apprenants à se présenter pour mieux se } \\
\text { - Lonnaître. } \\
\text { - } \text { attentem demander de faire part de leur motivation, de leurs } \\
\text { - Fournir des informations sur la composition et le profil } \\
\text { du groupe. } \\
\text { - Publier les coordonnées des apprenants pour faciliter les } \\
\text { communications sociales informelles. } \\
\text { - Rappeler le but commun pour renforcer le consensus. } \\
\text { - Encourager les apprenants à valider leurs connaissances } \\
\text { - Euprès du groupe. } \\
\text { - } \text { percourager les échanges sur le sens, l'utilité et la } \\
\text { - Lancer des appels à l'aide. } \\
\text { - Identifier les ressources et les compétences au sein du } \\
\text { - Aroupe. } \\
\text { Assurer la complémentarité des rôles pour la réalisation } \\
\text { de tâches communes. }\end{array}$ \\
\hline $\begin{array}{l}\text { Nature cognitive de la } \\
\text { tâche et ses exigences } \\
\text { La tâche consiste à } \\
\text { manipuler des idées } \\
\text { abstraites et des } \\
\text { concepts; elle exige } \\
\text { des habiletés } \\
\text { cognitives et } \\
\text { métacognitives de haut } \\
\text { niveau. }\end{array}$ & $\begin{array}{l}\text { - Expliquer clairement les exigences de la tâche } \\
\text { collaborative: } \\
\text { - les habiletés requises incluant celles à développer, } \\
\text { - la participation attendue, } \\
\text { - l'investissement personnel, } \\
\text { - les contraintes du travail intellectuel en groupe. } \\
\text { - Donner des conseils méthodologiques. } \\
\text { - Reconnaître les contributions et les efforts de chacun. } \\
\text { - Montrer comment les contributions cognitives de chacun } \\
\text { concourent à l'atteinte du but commun. }\end{array}$ \\
\hline $\begin{array}{l}\text { Besoins cognitifs } \\
\text { relatifs à la tâche } \\
\text { Les apprenants font un } \\
\text { retour sur leurs acquis, } \\
\text { évaluent la tâche et } \\
\text { élaborent des stratégies } \\
\text { pour la réaliser. }\end{array}$ & $\begin{array}{l}\text { - Fournir des outils d'autoévaluation. } \\
\text { - Fournir des outils d'autogestion. } \\
\text { - Présenter des habiletés métacognitives et en discuter. } \\
\text { - Sensibiliser à la pensée réflexive. }\end{array}$ \\
\hline $\begin{array}{l}\text { Espace commun, } \\
\text { partage des ressources } \\
\text { Collaborer, c'est } \\
\text { élaborer une } \\
\text { compréhension } \\
\text { partagée dans un } \\
\text { espace virtuel } \\
\text { commun. }\end{array}$ & $\begin{array}{l}\text { - Permettre à tous de prendre connaissance du travail } \\
\text { accompli. } \\
\text { - Inviter les apprenants à partager les ressources, } \\
\text { à échanger leurs travaux, à les comparer et à les } \\
\text { commenter dans le but d'aider et d'apprendre. }\end{array}$ \\
\hline
\end{tabular}


Pour développer le sentiment d'appartenance, les apprenants ont besoin de percevoir la présence des autres dans l'environnement et de se percevoir eux-mêmes dans le groupe (De la Teja, Lundgren-Cayrol et Paquin, 1997). Il ont aussi besoin de savoir qui fait quoi pour apprécier l'effort fourni par les autres, pour mesurer la qualité et la quantité du travail accompli par le groupe et pour s'assurer que l'engagement est mutuel. Ils doivent développer une compréhension partagée en travaillant ensemble dans un espace commun, en partageant leurs ressources et en s'entraidant (Schrage, 1990 ; Crotty, 1995 ; Abrami et al., 1995).

\section{Ressources de l'environnement technologique}

Les ressources de l'environnement doivent, de manière concrète, renseigner les apprenants sur leur propre contribution au travail de groupe et sur l'activité du groupe. Ce besoin peut être satisfait par la visualisation ou la représentation d'objets se rapportant aux participants, au contenu, à la tâche, aux interactions, aux ressources, etc. De plus, comme nous l'avons déjà montré, les apprenants doivent pouvoir évoluer dans des espaces de travail virtuels spécifiquement aménagés, où ils peuvent échanger, interagir et travailler ensemble à la réalisation de la tâche.

Les mesures sociométriques. Des représentations visuelles faisant état de la collaboration peuvent être générées par des outils d'analyse de la trace. Les sociogrammes, par exemple, sont utiles pour permettre à l'apprenant de savoir ce qui se passe au sein du groupe, d'apprécier la dynamique des échanges et de se faire une idée du degré d'engagement des uns et des autres (St-Arnaud, 1989 ; Dimock, 1985). Ce genre de représentation peut être obtenue à l'aide de dispositifs automatisés de mesure sociométrique intégrés au logiciel de forum électronique. La figure 3.4 fournit un exemple de sociogramme où l'on peut observer que l'apprenant B interagit peu avec le groupe. Une telle information peut être utile pour diagnostiquer un problème de fonctionnement au sein du groupe même à un stade précoce. Le formateur pourra alors planifier des interventions par exemple pour améliorer le climat, stimuler l'engagement ou encore pour aider un apprenant à participer plus activement. Lorsque ces représentations sont accessibles aux apprenants, il est conseillé de préserver l'anonymat comme dans l'exemple qui suit. 
Figure 3.4

INTERACTIONS AU SEIN D'UN GROUPE D'APPRENANTS

\section{Les espaces de travail virtuels}

La démarche collaborative invite les apprenants à s'engager dans une série de tâches collectives et individuelles. En conséquence, l'environnement doit non seulement offrir les outils appropriés pour les réaliser, mais aussi comporter des espaces de travail pour réaliser chaque type de tâches. L'apprenant doit pouvoir naviguer aisément d'un espace à l'autre, sentir qu'il n'est pas seul à occuper ces espaces et pouvoir observer que d'autres travaillent aussi à la réalisation de la tâche (Gutwin et al., 1995).

En s'appuyant sur une métaphore efficace, les interfaces doivent faciliter l'orientation dans les différents espaces, permettre de savoir qui est présent et qui est venu, et indiquer où en est la démarche collaboratif. L'environnement doit donc donner accès à un ensemble de renseignements sur la situation de collaboration qui pourront aider le groupe à poursuivre son travail.

- Qui fait ou a fait quoi, quand et comment (gestion des tâches)?

- Quels sont les résultats de la tâche et les productions qui en découlent? Dans quel espace ont-elles été déposées (partage des ressources)?

- Quelles productions sont en attente de commentaires (discussion, négociation, consensus)?

- Où en est le groupe et où en sont les équipes par rapport à l'échéancier?

- etc. 
Figure 3.5

EXEMPLES D'INTERFACES INDIQUANT LA PRÉSENCE DES PAIRS

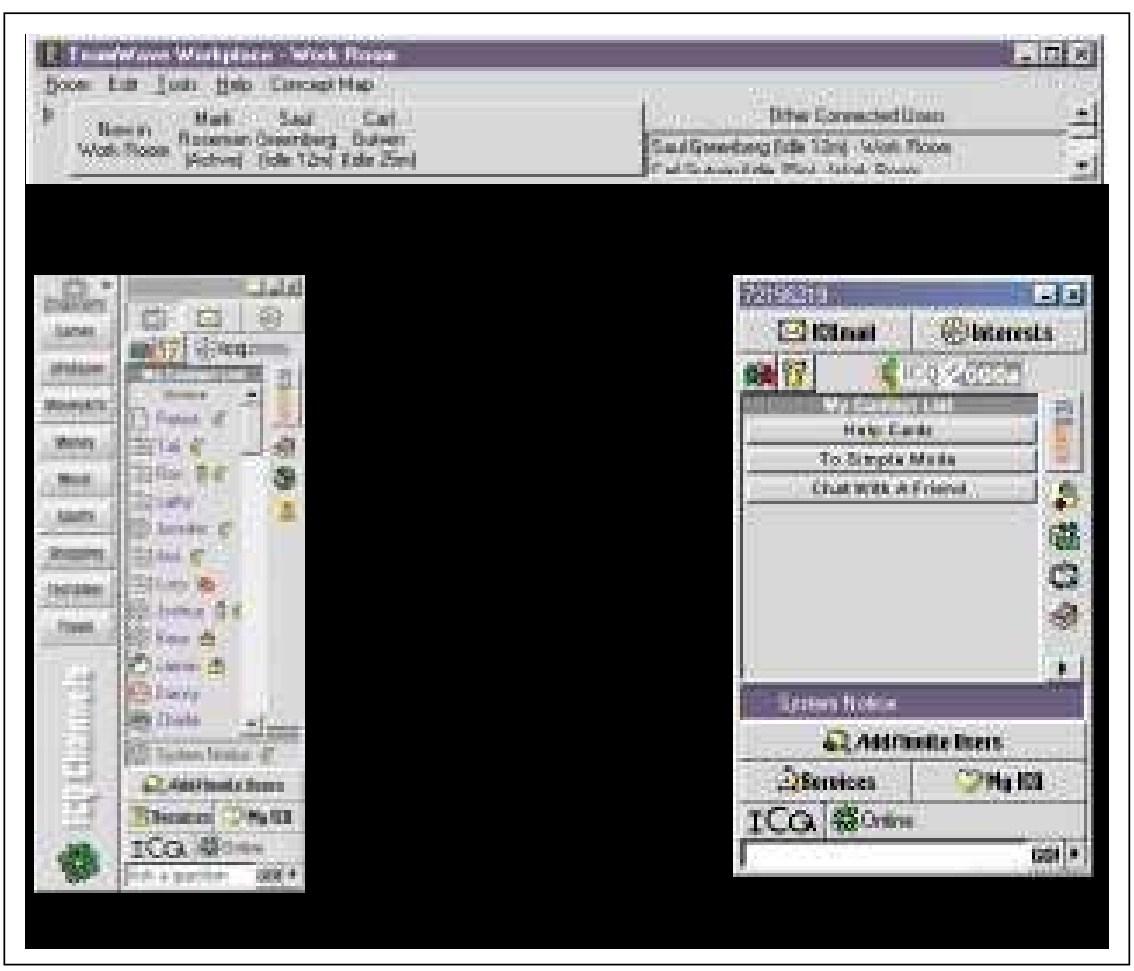

La figure 3.5 donne une idée de ce que les interfaces pourraient fournir comme renseignements.

\section{LA COHÉSION ET LA PRODUCTIVITÉ}

Comme nous l'avons mentionné au deuxième chapitre, la cohésion et la productivité sont des notions utiles pour décrire le fonctionnement d'un groupe virtuel ou présentiel et l'engagement des apprenants à l'égard du groupe. Nous verrons ici la corrélation entre les deux.

\section{La cohésion}

La cohésion s'observe et se mesure à travers la présence et l'absence d'un ensemble de comportements dans le groupe. La présence de comportements perçus de manière positive et, simultanément, 
l'absence d'autres comportements perçus de manière négative révèlent habituellement une bonne cohésion au sein du groupe (Hill, 1969 ; Dimock, 1987 ; St-Arnaud, 1989 ; Abrami et al., 1995 ; Lundgren-Cayrol, 1996). Un exemple de cette combinaison de comportements est donné au tableau 3.5.

Comme chaque groupe développe sa propre culture, les mêmes comportements peuvent être perçus différemment d'un groupe à l'autre. Chez un groupe, ce sera jugé comme un comportement souhaitable et dont la présence est positive, pourra être perçu comme indésirable ou négatif par un autre. Par exemple, l'humour peut être fort prisé chez certains groupes alors que chez d'autres, il n'est pas vu d'un bon œil. Pour stimuler et préserver la cohésion, il est donc utile pour les apprenants de connaître les perceptions du groupe afin qu'ils puissent adapter leurs comportements en conséquence.

En travaillant avec des groupes présentiels de jeunes, Kagan (1992) a observé qu'il est possible de stimuler la cohésion d'un groupe. Ils proposent aux éducateurs d'organiser des activités de

Tableau 3.5

\section{EXEMPLE DE COMPORTEMENTS INDIQUANT UNE BONNE COHÉSION DU GROUPE}

\begin{tabular}{|l|l|}
\hline $\begin{array}{l}\text { PRÉSENCE (comportements perçus } \\
\text { de manière positive) }\end{array}$ & $\begin{array}{l}\text { ABSENCE (comportements perçus } \\
\text { de manière négative) }\end{array}$ \\
\hline Partager de nouvelles informations. & Dominer la discussion. \\
\hline $\begin{array}{l}\text { Poser des questions pour mieux } \\
\text { comprendre. }\end{array}$ & Critiquer. \\
\hline Donner des explications. & Être compétitif. \\
\hline Fournir des exemples. & Être défensif. \\
\hline $\begin{array}{l}\text { Amorcer la discussion d'un nouveau sujet } \\
\text { qui fera véritablement avancer le travail. }\end{array}$ & Être hostile. \\
\hline S'exprimer avec humour au bon moment. & Être méchant. \\
\hline $\begin{array}{l}\text { Faire des commentaires positifs sur les } \\
\text { contributions des pairs. }\end{array}$ & Être sarcastique. \\
\hline
\end{tabular}


croissance d'appartenance dont l'objectif explicite est d'amener le groupe à se connaître et à comprendre comment travailler ensemble (par exemple, activités de prise de contact; activités d'appréciation menant au développement d'une interdépendance positive; activités normatives pour définir un but ou formuler des règles de participation, etc.). Il faut admettre cependant que ces interventions exigent beaucoup de temps et que ce n'est qu'après de nombreuses discussions que le groupe y arrive. Avec des groupes virtuels, certains outils peuvent être mis à la disposition des apprenants pour les aider à se connaître et à développer la cohésion comme nous le verrons plus loin (Ressources de l'environnement technologique, p. 121).

\section{La productivité}

L'évaluation de la productivité d'un groupe est également une question de perception: perception qu'ont les apprenants de la collaboration comme moyen d'atteindre le but commun et leurs objectifs personnels. Collings et Walker (1995) ont observé que lorsque le but commun est confus, le groupe ne sait pas véritablement à quoi sert ce qu'il fait ni où ses activités le mèneront. La cohésion se détériore et le groupe n'a pas l'impression d'être productif. Ces auteurs concluent que, pour éviter une telle situation qui met la collaboration en péril et pour stimuler la productivité, les groupes ont besoin d'expliciter et de négocier le but commun et de concilier les attentes des membres. Abrami et al. (1995), pour leur part, proposent d'influencer la perception de la productivité en donnant des notes de groupe plutôt que des notes individuelles. Cette méthode, utilisée avec de jeunes apprenants, n'a pas le même effet chez les apprenants matures et autonomes. Avec ces derniers, il vaudrait mieux négocier le but et la tâche à réaliser et s'entendre sur les modalités précises de réalisation: qui fait quoi, quand, comment, avec quoi, etc. Les participants auraient alors en main toutes les données pour apprécier la productivité réelle du groupe (Lundgren-Cayrol, 1996 ; Collings et Walker, 1995). 


\section{Cohésion et productivité}

Une recherche menée par Mullen et Copper (1994) a montré que la cohésion et la productivité sont étroitement liées et qu'elles s'influencent mutuellement. Pour ces auteurs, la cohésion est une résultante de quatre phénomènes: le pouvoir d'attraction du groupe sur les participants; l'intérêt que représente la tâche à accomplir; l'engagement dans l'accomplissement de la tâche et la fierté collective qui émane du groupe. Ils ont trouvé que l'engagement envers la tâche à accomplir est la variable la plus importante de la cohésion et de la productivité. Elle produit un effet positif sur la performance du groupe, et les informations que le groupe reçoit sur cette performance influencent de façon positive sa cohésion. En outre, ils ont observé que la corrélation s'applique, encore davantage, lorsqu'il s'agit de petits groupes.

Figure 3.6

\section{CORRÉLATION POSITIVE ENTRE LA COHÉSION ET LA PRODUCTIVITÉ DU GROUPE}

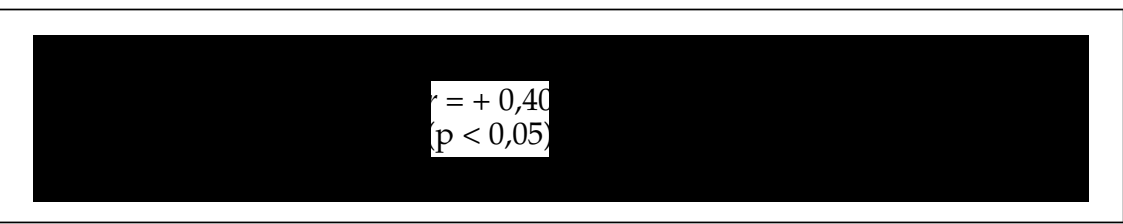

Source : Lundgren-Cayrol, 1996.

Abrami et ses collaborateurs (1995) ont développé une méthode pour informer le groupe de sa performance en utilisant des indices de productivité. Conçue pour des situations en face à face, cette méthode invite les apprenants à remplir pendant et après les séances de travail des questionnaires sur leur perception de la cohésion et de la productivité du groupe. Tout en préservant l'anonymat des répondants, les résultats sont présentés au groupe. Ces chercheurs ont observé que cette stratégie induit chez les apprenants une prise de conscience du fonctionnement du groupe et les amène à réfléchir sur leur manière de collaborer. Cette réflexion agit sur le fonctionnement du groupe et elle a comme effet d'améliorer le climat de travail, les conditions d'apprentissage, la cohésion et la productivité. 


\section{RESSOURCES DE L'ENVIRONNEMENT TECHNOLOGIQUE}

Les recherches sur la cohésion et la productivité des groupes d'apprenants nous amènent à postuler que pour optimiser l'apprentissage collaboratif à distance, les environnements virtuels doivent intégrer des outils de socialisation et de suivi de la tâche ainsi que des instruments de mesure de la cohésion et de la productivité.

Pour stimuler la cohésion, on peut prévoir des outils qui vont faciliter et même stimuler la socialisation (Wells, 1995; Berge, 1995 ; Collings et Walker, 1995). En voici des exemples.

- Un répertoire des participants fournissant les coordonnées de chacun renseignera les participants sur la composition et la taille du groupe (Qui sont mes pairs?).

- Un formulaire de carte de visite que chacun remplit pour se présenter au groupe (objectifs, aspirations, intérêts et moyens de les joindre, etc.) facilitera les premiers contacts, stimulera l'intérêt mutuel et favorisera le sentiment d'interdépendance (pouvoir d'attraction du groupe).

- Un espace pour le dépôt des curriculum vitæ peut faciliter la formation des équipes et aider à apprécier les compétences et la complémentarité des membres du groupe (réseau d'entraide).

- Un espace de socialisation pour bavarder de tout et de rien contribue à l'établissement d'un climat sain et de camaraderie (sentiment d'appartenance).

Pour aider les apprenants à évaluer la productivité du groupe, on peut offrir des outils de suivi de projet qui montrent clairement l'avancement de la tâche. On peut également mettre à la disposition des apprenants divers instruments de mesure automatisés (questionnaires électroniques, instruments de sondage, mécanismes de vote, etc.) et prévoir des modules d'aide à l'interprétation des résultats. Pour bâtir ces instruments, on pourra s'inspirer de ceux qui ont été développés pour étudier la cohésion et la productivité du groupe (voir les instruments développés par Abrami et al., 1995; Lundgren-Cayrol, 1996; Mullen et Copper, 1994 ; St-Arnaud, 1989 et Dimock, 1985). Ces instruments sont des inventaires de comportements que l'on soumet 
Tableau 3.6

\section{EXEMPLE DE RÉSULTATS DE L'ÉVALUATION DE LA PRODUCTIVITÉ D'UN GROUPE}

\begin{tabular}{|ll|}
\hline Pour le développement des idées... \\
\hline $81 \%$ & Nous n'avons rien fait ou presque pour générer des idées nouvelles. \\
$10 \%$ & $\begin{array}{l}\text { Nous nous sommes fait imposer des idées par un ou deux membres } \\
\text { du groupe. }\end{array}$ \\
$9 \%$ & Nous nous encourageons les uns les autres. \\
\hline
\end{tabular}

aux membres du groupe à divers moments et sur lesquels chaque apprenant se prononce au cours de la réalisation du travail collectif. Les résultats fournissent des indicateurs du climat. Le groupe et le formateur peuvent en tirer des pistes d'action pour améliorer la cohésion et la productivité. Le tableau 3.6 donne un exemple de résultats indiquant un problème de performance.

D'autres dispositifs moins spécialisés, comme ceux que nous avons mentionnés plus haut pour soutenir le sentiment d'appartenance, peuvent aussi être utilisés pour aider le groupe à atteindre une meilleure productivité. Des guides de réalisation de tâche, des indicateurs de nouveaux messages ou de nouveaux documents sont autant de moyens qui peuvent soutenir la productivité en renseignant l'apprenant sur ce qu'il y a à faire, ce qui a été fait et ce qui reste à faire.

\section{LA COORDINATION}

Coordonner, c'est voir à l'agencement efficace des activités, des personnes et des ressources pour atteindre une fin. Dans un contexte de collaboration, coordonner, c'est prendre en charge la gestion de la tâche: découpage en sous-tâches, attribution des responsabilités, utilisation des ressources, etc. C'est aussi gérer les aspects affectifs et psychosociaux du groupe: reconnaissance de chacun, soutien, encouragement, motivation, climat, etc. Pour décrire le processus de coordination dans un environnement virtuel, nous reprenons la stratégie développée par Ricciardi-Rigault et Henri (1989) qui porte principalement sur l'organisation du travail du groupe et sur l'architecture des forums électroniques. 


\section{L'ORGANISATION DU TRAVAIL DU GROUPE}

Ricciardi-Rigault et Henri ciblent trois variables à contrôler afin de mieux canaliser et de coordonner les énergies et les activités du groupe: 1) la tâche, 2) la constitution et la composition du groupe et 3) l'animation.

\section{La tâche}

Réaliser une tâche collaborative, c'est travailler ensemble et s'entraider pour que chacun atteigne le but que le groupe s'est fixé en négociation et en tenant compte des attentes de chacun. La tâche mène à une production concrète et engage les trois composantes du modèle de collaboration que nous avons présenté plus haut: communication, engagement et coordination. Elle est découpée et organisée en trois sous-tâches génériques, négocier, réaliser et gérer, présentées au tableau 3.7.

Tableau 3.7

\section{DÉCOUPAGE DE LA TÂCHE}

\begin{tabular}{|l|l|}
\hline Découpage de la tâche & Composantes de la collaboration \\
\hline $\begin{array}{l}\text { 1. Discuter, négocier pour s'entendre sur } \\
\text { le projet à réaliser. }\end{array}$ & Communication des idées \\
\hline $\begin{array}{l}\text { 2. } \\
\text { Réaliser le projet en travaillant } \\
\text { ensemble. }\end{array}$ & Engagement à l'égard du groupe \\
\hline $\begin{array}{l}\text { 3. } \\
\begin{array}{l}\text { Gérer la réalisation du projet en } \\
\text { fixant les paramètres matériels, } \\
\text { temporels, spatiaux et } \\
\text { organisationnels du travail. }\end{array}\end{array}$ & Coordination \\
\hline
\end{tabular}

\section{Le groupe et les équipes}

La constitution et la composition du groupe ou des équipes représentent une étape critique qu'il ne faut pas négliger pour assurer un fonctionnement optimal de la collaboration. C'est un moment critique et délicat qui peut influencer la motivation et l'engagement. C'est pourquoi il est souhaitable que les apprenants comprennent les choix qui auront été faits au regard de la constitution du groupe ou des équipes. Le groupe ou les équipes seront-ils homogènes? Qui sera responsable de les composer? 
Quelle sera leur taille? Quelle méthode sera adoptée pour répartir les apprenants dans les groupes ou dans les équipes? Le formateur répondra d'abord à ces questions en considérant les données sociodémographiques usuelles sur les apprenants, les éléments de contexte, les objectifs d'apprentissage et le modèle de collaboration souhaité.

Il y a essentiellement deux méthodes pour constituer des groupes et des équipes: l'assignation ou le libre choix. Si les apprenants se connaissent, s'ils ont l'habitude de travailler en groupe et si le temps le permet, il est souvent préférable de les laisser se regrouper. Dans le cas contraire, il est préférable de répartir les apprenants au hasard. Le temps dont on dispose pour faire l'activité de collaboration et le type de travail à réaliser sont des facteurs déterminants de la méthode à adopter pour constituer le groupe (Abrami et al., 1995 ; Lundgren-Cayrol, 1996).

Tableau 3.8

\section{ProblématiQue de la CONSTITUTION} ET DE LA COMPOSITION D'UN GROUPE

\begin{tabular}{|c|c|}
\hline $\begin{array}{l}\text { Constitution et composition du } \\
\text { groupe ou des équipes }\end{array}$ & Aspects à considérer \\
\hline $\begin{array}{l}\text { Le groupe sera-t-il hétérogène ou } \\
\text { homogène? }\end{array}$ & $\begin{array}{l}\text { Cela dépend des aspects que l'on veut favoriser. } \\
\text { - Complémentarité, interdépendance } \\
\text { - Cohésion } \\
\text { - Productivité }\end{array}$ \\
\hline $\begin{array}{l}\text { Le formateur choisit-il seul les } \\
\text { membres du groupe et des } \\
\text { équipes? } \\
\text { Le choix est-il laissé à la discrétion } \\
\text { des apprenants? }\end{array}$ & $\begin{array}{l}\text { Cela dépend du profil des apprenants et du temps } \\
\text { dont on dispose pour effectuer le regroupement. } \\
\text { - Autonomie } \\
\text { - Culture du milieu } \\
\text { - Contraintes temporelles }\end{array}$ \\
\hline $\begin{array}{l}\text { Quelle sera la taille du groupe: } \\
\text { petite, moyenne ou grande? }\end{array}$ & $\begin{array}{l}\text { Cela dépend de la tâche. } \\
\text { - Discussion = grands groupes (25-30) } \\
\text { - Réalisation du projet = groupes plus petits } \\
\text { (équipes, 3-5) } \\
\text { - Gestion = groupes de taille moyenne (moins } \\
\text { de } 20 \text { ) } \\
\text { Cela dépend de ce qu'on attend du groupe en termes } \\
\text { d'engagement, de participation. } \\
\text { - Plus le groupe est grand, moins les pressions } \\
\text { sont fortes au regard de l'engagement et de } \\
\text { la participation. }\end{array}$ \\
\hline
\end{tabular}




\section{L'animation}

L'animation des forums électroniques joue un rôle de première importance pour la réussite de la collaboration à distance. Pour chaque forum, la présence d'un modérateur est indispensable ; il faudra décider qui jouera ce rôle. Pour chaque groupe, il faudra choisir et planifier la stratégie d'animation. C'est le formateur qui est le principal responsable des décisions à prendre en matière d'animation. Il lui faut déterminer la personne qui animera les débats ou le travail et la stratégie d'animation qui sera adoptée. Cependant, plus le groupe est autonome, plus il sera en mesure d'assurer sa propre animation. Le tableau 3.9 reprend trois éléments de cette problématique que nous avons déjà présentée dans le deuxième chapitre: les fonctions et les stratégies d'animation, une typologie des interventions et le choix du modérateur.

\section{Tableau 3.9}

\section{ANIMATION DES FORUMS ÉLECTRONIQUES}

\begin{tabular}{|l|l|}
\hline $\begin{array}{l}\text { Éléments de la problématique de } \\
\text { l'animation des forums }\end{array}$ & Éléments de réponse \\
\hline $\begin{array}{l}\text { Quelles fonctions et quelles } \\
\text { stratégies d'animation? }\end{array}$ & $\begin{array}{l}\text { - Hôte social } \\
\text { - Guide, facilitateur, orienteur, gestionnaire } \\
\text { - Encouragement à la participation } \\
\text { - Soutien à la motivation }\end{array}$ \\
\hline Quelles interventions? & $\begin{array}{l}\text { Feenberg (1989) en propose trois types. } \\
\text { - La contextualisation: contexte et structure de } \\
\text { la discussion, calendrier de travail, mode de } \\
\text { fonctionnement des échanges, procédure, } \\
\text { code d'éthique, etc. } \\
\text { - Le monitoring: vérification de la cohésion et } \\
\text { de la productivité, reconnaissance de la } \\
\text { participation, guidage de la tâche à } \\
\text { accomplir, etc. } \\
\text { - Les métafonctions: ouverture et clôture, } \\
\text { résumé ou conclusion, liens entre les sous- } \\
\text { tâches, liens entre les idées (macrostructure } \\
\text { et microstructure), etc. }\end{array}$ \\
\hline Qui jouera le rôle de modérateur? & $\begin{array}{l}\text { Cette décision est prise en fonction de la tâche } \\
\text { et de la taille du groupe. } \\
\text { - Pour une télédiscussion avec un grand } \\
\text { groupe: le formateur } \\
\text { - Pour une télégestion en groupe de taille } \\
\text { moyenne : le formateur } \\
\text { - Pour la réalisation du projet en équipe: un } \\
\text { apprenant }\end{array}$ \\
\hline
\end{tabular}




\section{RESSOURCES DE L'ENVIRONNEMENT TECHNOLOGIQUE}

\section{Pour le soutien à la tâche}

Tous les dispositifs que nous avons décrits précédemment contribuent au soutien à la tâche. Dans une perspective de coordination, il faut cependant s'assurer de les rendre disponibles au moment voulu - ils n'ont pas à être tous accessibles en permanence - et au bon endroit. Pour éviter la confusion et la désorientation, l'environnement devrait être évolutif s'il est le moindrement complexe. Il devrait se transformer et s'adapter aux besoins ponctuels des apprenants. La configuration de l'environnement devrait aussi aider le groupe à développer une vision partagée de la collaboration, stimuler l'engagement et contrer le sentiment d'isolement. À cet effet, pour une activité ou une tâche donnée, l'interface devrait permettre d'afficher automatiquement l'état d'avancement du travail et pointer vers les forums correspondants.

\section{La constitution du groupe et des équipes}

Selon la méthode retenue pour constituer le groupe, l'assignation ou le libre choix, les outils pourront varier. Voici quelques outils qui peuvent soutenir la constitution des groupes.

- Une liste des membres d'un groupe fournissant leurs coordonnées et, éventuellement, les données sociodémographiques usuelles: plutôt utile pour la méthode par assignation;

- Une «carte de visite » qui permet à chacun de se présenter : plutôt utile pour la méthode par libre choix;

- Un indicateur des présences en ligne pour faciliter et encourager les premiers contacts: plutôt utile pour la méthode par libre choix.

\section{L'animation}

Pour assister l'animateur dans sa tâche, que ce rôle soit joué par le formateur ou un apprenant, le logiciel de forum électronique doit offrir une variété d'outils de gestion pour:

- créer des groupes;

- ouvrir et fermer les forums ; 
- accorder des privilèges et des droits d'accès ;

- suivre le déroulement des échanges: contenu, dynamique cognitive et dynamique psychosociale ;

- établir un calendrier et un agenda ;

- fabriquer des formulaires de suivi de la tâche ou des rapports d'avancement des travaux;

- élaborer des instruments de mesure, de sondage et de vote ;

- créer des outils de prise de décision.

L'animateur doit pouvoir combiner l'utilisation des outils de gestion avec celle de tous les autres outils présents dans l'environnement: outils de planification; de mesure sociométrique, d'analyse du contenu des échanges, de représentation du contenu sémantique; etc. Les informations que l'animateur obtiendra de ces ressources diverses l'aideront à apprécier la performance du groupe, à poser des diagnostics et à décider du type d'intervention appropriée à chaque situation.

\section{LA STRUCTURATION DE L'ESPACE DE COMMUNICATION}

La structure de l'espace de communication que nous avons présentée dans le deuxième chapitre retient cinq grandes espèces de forums qui ont chacune leurs caractéristiques et leur vocation propre: télédiscussion, télétravail, télégestion, téléassistance et télésocialisation. Parmi elles, nous nous intéressons ici plus spécifiquement aux trois espèces qui se rapportent à la tâche cognitive: télédiscussion, télétravail et télégestion.

La télédiscussion est utilisée pour soutenir les échanges intellectuels (débat, développement d'une problématique, élaboration d'hypothèses, etc.) qui auront une conclusion à l'intérieur du forum lui-même. Elle sert également à discuter et à alimenter un projet qui sera réalisé à l'extérieur du forum, dans d'autres espaces. Le forum de télétravail est le lieu où sont effectivement réalisés des projets communs. La télégestion sert à la communication de consignes, à la prise de décisions reliées à l'organisation et à la logistique de la tâche, et au suivi de l'ensemble du processus. Le tableau 3.10 présente les trois espèces de forum en rapport avec la tâche, la taille du groupe et l'animation. 
Tableau 3.10

ESPÈCES DE FORUMS EN RAPPORT AVEC LA TÂCHE, LA TAILLE DU GROUPE ET L'ANIMATION

\begin{tabular}{|l|l|l|l|}
\hline Variable & Télédiscussion & Télétravail & Télégestion \\
\hline Tâche & $\begin{array}{l}\text { Discuter pour } \\
\text { alimenter un } \\
\text { projet, commun } \\
\text { ou non, qui sera } \\
\text { réalisé à l'extérieur } \\
\text { du forum. }\end{array}$ & $\begin{array}{l}\text { Réaliser un projet } \\
\text { en commun, à } \\
\text { l'intérieur du } \\
\text { forum. }\end{array}$ & $\begin{array}{l}\text { Communiquer } \\
\text { des consignes. } \\
\text { Décider la } \\
\text { logistique. } \\
\text { Effectuer le suivi } \\
\text { de l'ensemble } \\
\text { du processus. }\end{array}$ \\
\hline Taille du groupe & $\begin{array}{l}\text { Plus grand groupe } \\
\text { (de 20 à 30 } \\
\text { au plus) }\end{array}$ & $\begin{array}{l}\text { Petit groupe } \\
\text { (3 à 5) }\end{array}$ & $\begin{array}{l}\text { Groupe de taille } \\
\text { moyenne } \\
\text { (20 au plus) }\end{array}$ \\
\hline Animation & Par le formateur & Par les apprenants & Par le formateur \\
\hline
\end{tabular}

\section{Ressources de l'environnement technologique}

C'est surtout l'interface qui entre en jeu lorsqu'il s'agit de représenter les lieux de communication. L'apprenant doit pouvoir identifier facilement à quel forum il doit se joindre pour la tâche à accomplir. Une interface simple doit permettre de reconnaître facilement la manière dont sont organisés les forums. Pour les télédiscussions, il s'agira de montrer la structure du thème ou de l'objet de discussion: linéaire, arborescente, parallèle, etc. Pour la télégestion, il faut identifier l'objet sur lequel porte la gestion. Enfin, pour le télétravail, chaque équipe devrait pouvoir repérer le lieu de travail qui lui est réservé.

\section{TABLEAU SYNTHÈSE DES RESSOURCES DE COLLABORATION}

Le tableau 3.11 fournit une liste de ressources de collaboration qui trouvent leur place dans une environnement d'apprentissage virtuel. Nous les avons mis en relation avec les composantes de notre modèle de collaboration. 
Tableau 3.11

\section{SYNTHÈSE DES RESSOURCES DE COLLABORATION}

\begin{tabular}{|c|c|c|}
\hline $\begin{array}{l}\text { Les composantes } \\
\text { de la collaboration }\end{array}$ & Le groupe comme ressource & $\begin{array}{l}\text { Les ressources technologiques dans le forum } \\
\text { et en périphérie }\end{array}$ \\
\hline $\begin{array}{l}\text { Communication des idées } \\
\text { - Microstructure } \\
\text { - Macrostructure }\end{array}$ & $\begin{array}{l}\text { Il est important que l'étudiant puisse: } \\
\text { - exprimer et communiquer librement ses idées ; } \\
\text { - faire émerger de nouvelles idées; } \\
\text { - faire des liens entre les idées pour élaborer des } \\
\text { concepts; } \\
\text { - construire ses propres connaissances. }\end{array}$ & $\begin{array}{l}\text { - outil d'aide à la structuration des messages; } \\
\text { - outil d'aide à la structuration de la discussion en } \\
\text { fonction de la tâche (activité cognitive, } \\
\text { socialisation, coordination); } \\
\text { - visualisation de l'organisation des forums; } \\
\text { - affichage de tous les messages pour que leur } \\
\text { lecture soit incontournable (pour favoriser la } \\
\text { participation); } \\
\text { - visualisation des liens entre les messages; } \\
\text { - à partir de n'importe quel point de } \\
\text { l'environnement, repérage facile et rapide du } \\
\text { forum à joindre pour envoyer un message; } \\
\text { - accès aux condensés des messages ; } \\
\text { - accès et repérage de l'information à l'aide d'outils } \\
\text { de fouille dans le texte des messages et dans tous } \\
\text { les autres documents ; } \\
\text { - outil hypertexte pour créer les liens ; } \\
\text { - outil d'annotations personnelles; } \\
\text { - résident local pour manipulation hors ligne. }\end{array}$ \\
\hline
\end{tabular}


Tableau 3.11

SYNTHÈSE DES RESSOURCES DE COLLABORATION (suite)

\begin{tabular}{|c|c|c|}
\hline $\begin{array}{l}\text { Les composantes } \\
\text { de la collaboration }\end{array}$ & Le groupe comme ressource & $\begin{array}{l}\text { Les ressources technologiques dans le forum } \\
\text { et en périphérie }\end{array}$ \\
\hline $\begin{array}{l}\text { Engagement } \\
\text { - cognitif } \\
\text { - affectif/social }\end{array}$ & $\begin{array}{l}\text { Pour assurer une collaboration efficace, les } \\
\text { membres d'un groupe d'apprenants doivent } \\
\text { atteindre un haut niveau de performance, de } \\
\text { cohésion sociale et de productivité } \\
\text { intellectuelle. } \\
\text { - Sur le plan socioaffectif, il faut qu'un climat de } \\
\text { confiance puisse s'établir et que les sources } \\
\text { d'anxiété soient éliminées. }\end{array}$ & $\begin{array}{l}\text { - analyseur de la trace; } \\
\text { - historique (qui a lu quoi et quand; qui a fait quoi, } \\
\text { - quand et comment); } \\
\text { instruments de mesure automatisés de la cohésion } \\
\text { - générateur de statistiques (indices de productivité } \\
\text { et de cohésion, par exemple); } \\
\text { - liste des participants et leurs CV, carte de visite; } \\
\text { - visualisation de la dynamique des échanges } \\
\text { (communicogramme, sociogramme). }\end{array}$ \\
\hline $\begin{array}{l}\text { Coordination } \\
\text { - télédiscussion } \\
\text { - télétravail } \\
\text { - télégestion }\end{array}$ & $\begin{array}{l}\text { L'organisation et la gestion des activités, tant } \\
\text { des grands que des petits groupes, doivent } \\
\text { être facilitées pour que l'apprentissage soit } \\
\text { harmonieux et efficace. }\end{array}$ & $\begin{array}{l}\text { - création et gestion faciles et efficaces des forums; } \\
\text { - orchivage et repérage de l'information; } \\
\text { (journal de bord, rapport d'avancement des } \\
\text { travaux, calendrier, etc.); } \\
\text { - outils de prise de décision (sondage, vote). }\end{array}$ \\
\hline
\end{tabular}




\section{Chapitre 4}

\section{La dynamique de l'apprentissage collaboratif}

$\mathrm{N}$

ous avons vu jusqu'ici que la démarche collaborative s'appuie sur les théories sociocognitives pour préconiser un processus de re-construction des connaissances. Dans un contexte de formation à distance, soutenue par un modèle de collaboration fait de communication d'idées, de coordination et d'engagement, l'apprentissage collaboratif s'actualise à travers l'usage d'environnements virtuels aménagés en espaces et en lieux stratégiques d'interaction où les apprenants disposent d'une variété de ressources, d'outils et de moyens pour atteindre le but commun tout en satisfaisant leurs propres attentes et leurs propres besoins.

Dans ce dernier chapitre, nous complétons notre étude en décrivant les phases de la collaboration après avoir analysé la dynamique de l'interaction cognitive qui se joue entre quatre principaux acteurs: l'apprenant, le groupe, le formateur et l'expert de contenu. Nous nous intéressons par la suite aux habiletés cognitives et de collaboration que l'apprenant doit maîtriser pour participer pleinement à la démarche collaborative. Puis nous nous penchons sur le rôle du formateur qui 
accompagne l'apprenant et dont la mission est de le faire progresser dans son cheminement vers une plus grande autonomie et une plus grande responsabilisation face à son apprentissage. Finalement, nous abordons le problème de l'évaluation des apprentissages dont la complexité est aussi grande que l'apprentissage lui-même.

\section{LA DYNAMIQUE COGNITIVE DE L'APPRENTISSAGE COLLABORATIF}

Selon les théories sociocognitives, l'apprentissage résulte d'interactions sociales et cognitives, comme celles qu'on peut observer entre le maître et l'apprenti. L'apprenti observe le maître, l'interroge, discute avec lui et suit ses conseils. Le maître commente, critique et corrige le travail de l'apprenti; il le guide et l'initie à son savoir-faire. De manière intentionnelle et progressive, il met à la disposition de l'apprenti ses outils, ses méthodes et ses techniques (Lave et Wenger, 1991). Ce faisant, l'apprenti prend conscience $\mathrm{du}$ rapport que le maître entretient avec son milieu et découvre graduellement l'utilité sociale du travail du maître. L'apprenti acquiert la conviction que ce qu'il apprend à faire a un sens et une portée.

Pour renforcer le principe de l'ancrage social des connaissances, Linard (1995) et Laurillard (1995) nous rappellent, en se référant aux théories sociales d'apprentissage de Piaget, Vygotsky et Leontiev, qu'une connaissance n'est véritable que si elle a pu être exprimée, explicitée, mise en contexte et confrontée à d'autres. Boder (1993) soutient également ce principe d'ancrage social en expliquant que les connaissances ne sont que de l'information qui deviendra connaissance dans la mesure où l'apprenant réussit à l'intégrer dans un usage.

Dans un environnement d'apprentissage, il y a donc quatre entités qui interagissent: le milieu en tant qu'agent de sens; le maître en tant qu'expert, détenteur des connaissances et agent de communication du savoir socialement reconnu; le maître en tant que formateur, guide et accompagnateur; et l'apprenant en tant qu'agent de son propre changement. Au départ, pour l'appre- 
nant, les connaissances du maître, expert de contenu, ne sont encore que de "l'information ». Ce n'est qu'après avoir appris à utiliser l'information dans un contexte réaliste et à la mettre au service d'usage que l'apprenant pourra dire qu'il a véritablement appris.

L'apprenant arrive à transformer l'information en connaissances véritables, utilisables, dans la mesure où il y voit un sens et une portée sociale (Chan et Chou, 1995 ; Choi et Hannafin, 1995 ; Appelt, 1995 ; Berge, 1995). Cela veut dire, pour lui, qu'il comprend à quel besoin social elles répondent et qu'il sait effectivement comment s'en servir dans la réalité. Cette démarche menant à la découverte du sens à donner aux apprentissages pose un défi de taille pour le formateur, surtout lorsqu'elle porte sur un contenu abstrait ou théorique dont l'application dans un usage n'est pas toujours évident ou immédiat. Ce défi est aussi celui du concepteur d'environnements d'apprentissage virtuels et collaboratifs qui doit imaginer non seulement un système d'interactions purement cognitives favorisant la transformation de l'information en connaissances, mais aussi un système d'interactions humaines et sociales indispensable au développement des communautés d'apprenants. Pour que la dynamique cognitive puisse s'épanouir au sein d'un groupe, il doit y régner un climat social sain empreint de motivation, du sentiment d'appartenance, d'engagement et de solidarité. Le concepteur doit donc concevoir des environnements qui mobilisent les apprenants et qui les amènent à s'engager à apprendre et à construire leurs connaissances ensemble.

Dans ces environnements, deux systèmes d'interaction sont en jeu : le social et le cognitif. Ils ne sont pas étanches et ils s'interinfluencent. Leur chimie, à chaque fois différente, fait qu'un groupe peut développer une synergie et ainsi faire en sorte de produire plus que ce qu'un individu pourrait faire seul. Si nous faisons ici l'exercice de distinguer les interactions sociales et cognitives, c'est parce que, dans la médiatisation des environnements d'apprentissage virtuels, le concepteur doit tenir compte de ces deux dimensions de la collaboration pour assurer une juste place autant à l'une qu'à l'autre. 
Sans minimiser l'importance des facteurs humains et sociaux de l'apprentissage (attitudes, motivation, climat social, culture du groupe, etc.), nous poursuivons notre étude en nous limitant à la description cognitive des quatre acteurs de l'apprentissage et du système qui les fait interagir.

\section{LES QUATRE PRINCIPAUX ACTEURS DE L'INTERACTION COGNITIVE}

La dynamique cognitive de l'apprentissage met en présence quatre acteurs que nous allons tenter ici de situer. Ce sont 1) le modèle de connaissances de l'expert, 2) l'apprenant, 3) le groupe, et 4) le formateur qui interagissent dans une dynamique globale convergeant vers l'apprentissage.

\section{Le modèle de connaissances de l'expert}

Dans un environnement d'apprentissage, le modèle de connaissances est un corpus qui représente le savoir socialement reconnu; c'est l'objet à connaître, l'objet d'étude, la matière, le contenu d'apprentissage. Il est réputé être le fait de l'expert. Nous le proposons comme acteur du processus d'apprentissage parce qu'à travers lui, c'est la société qui s'exprime ; parce qu'il influence la société et qu'il peut être influencé par elle. Un modèle de connaissances n'est pas une entité stable. Appelé à changer en fonction de l'avancement des connaissances, de la réflexion et de l'évolution de la société, il traduit une vision du monde incarnée dans le temps. Par lui, l'expert et, implicitement, la société proposent une structure et une organisation des connaissances que l'apprenant et le groupe, aidés par le formateur, vont explorer, appréhender, critiquer et reconstruire.

\section{L'apprenant}

Au départ, comme nous l'avons dit plus haut, les connaissances de l'expert ne sont encore pour l'apprenant que des informations avec lesquelles il interagit afin d'en appréhender la structure, 
l'étendue, le sens et l'utilité. Dans un dialogue intérieur qui mobilise ses capacités d'analyse, de synthèse et de penseur critique, il élabore ses propres représentations. Cette première phase, qui en est une d'exploration, se poursuit collectivement avec les membres du groupe. Dans l'espace de communication, l'apprenant rend explicites ses premières constructions cognitives en exprimant ses idées. Il découvre d'autres modèles de connaissances que le sien, ceux de ses pairs, membres du groupe. Son modèle, plus ou moins élaboré, plus ou moins juste, va être transformé, refait et reconstruit tout au long de ses recherches d'information, de sa réflexion personnelle et des interactions avec le groupe. Les échanges de l'apprenant avec le groupe ne sont pas à sens unique; il reçoit mais il donne aussi.

\section{Le groupe}

Des discussions et des débats qui ont lieu entre les apprenants au cours de la collaboration se dégage une intelligence collective, une entité qui a son existence propre. Le groupe est une entité qui naît, croît et se développe par le jeu des rapports et des interactions entre ses membres. Il se donne des règles, invente sa propre organisation, distribue les rôles et travaille pour atteindre un but commun. Chaque groupe développe sa propre culture et se caractérise par son degré de maturité et d'engagement cognitif. L'intelligence collective et les artefacts qu'il produit lui permettent d'exercer son activité. Dans les environnements virtuels, le groupe, qui forme un collectif intelligent, se rassemble pour explorer et s'approprier le modèle de connaissances de l'expert. Tout au long des échanges et des discussions, les membres partagent l'information, la structurent et l'organisent de manière cohérente et signifiante pour le groupe. Les productions du groupe sont plus qu'une simple accumulation des contributions ou des points de vue de chacun; elles traduisent un modèle de connaissances organisé, original et distinct de celui de l'expert et de chaque apprenant. 


\section{Le formateur}

Le «maître » et les «connaissances de l'expert» ont longtemps été confondus. Le maître était l'expert et les connaissances n'étaient accessibles qu'à travers lui. Il en était le possesseur, le porteur et le communicateur. Il développait les connaissances et il lui incombait de les enseigner. Aujourd'hui, cette conception qui fait du formateur un «maître» est ébranlée. L'étendue des connaissances est telle qu'un formateur ne peut plus toutes les posséder. En outre, il ne peut plus faire compétition aux moyens de communication qui rendent l'information accessible à tous de manière beaucoup plus efficace. Désormais, le concept qui fusionne «maître » et «connaissances de l'expert » a perdu de sa prégnance de même que la relation d'autorité qui la caractérisait.

Selon le paradigme constructiviste, le formateur n'a pas comme rôle principal de transmettre les connaissances; il n'est plus confondu avec le corpus de connaissances à acquérir. Il est vu comme un facilitateur et un animateur de l'interaction cognitive et sociale. Son rôle consiste, en éclaireur avisé, à structurer la démarche de recherche et de traitement de l'information par l'apprenant et par le groupe. Il les accompagne, les aide à donner un sens à leur travail, les encourage, les stimule et les motive. Il veille sur le groupe, recueille des informations sur son fonctionnement, pose des diagnostics, formule des hypothèses, élabore des stratégies d'interventions et les met en œuvre auprès des apprenants et du groupe. Il intervient comme médiateur entre les parties, préoccupé de la productivité cognitive du groupe mais aussi du climat social qui y règne.

L'interaction cognitive qui se produit entre ces quatre acteurs est complexe, car elle n'emprunte pas un processus linéaire ou séquentiel. Elle intègre les démarches individuelles et collective; elle s'appuie sur l'intériorisation et l'extériorisation des processus; elle est faite de recherche de sens et de constructions originales. Il s'agit d'un système d'interactions dynamique que nous avons tenté de représenter à la figure 4.1.

Dans cette dynamique cognitive de l'apprentissage, l'apprenant, mis en contact avec le modèle de connaissances de l'expert, s'engage dans un dialogue intérieur pour se forger une première 
Figure 4.1

APPRENDRE: INTÉGRER L'INFORMATION DANS UN USAGE

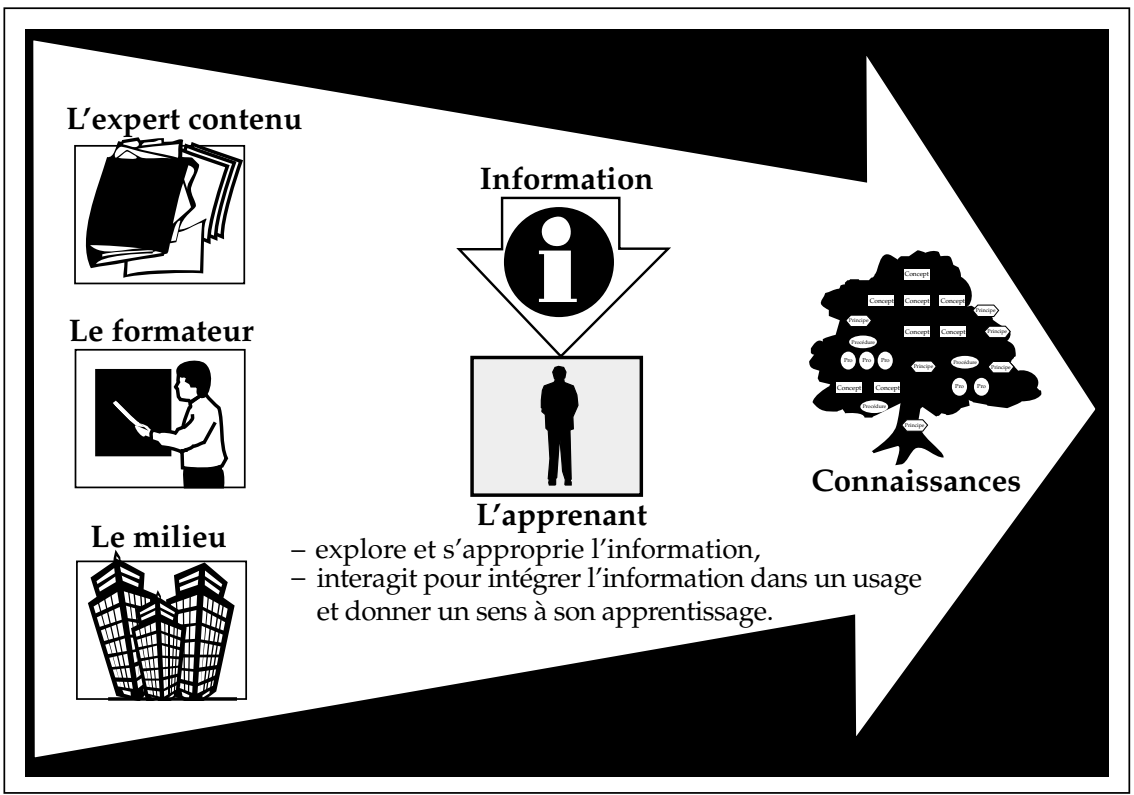

représentation de l'objet à connaître. En parallèle, le groupe s'alimente des contributions de chacun et du modèle de connaissances de l'expert. Il en tire l'information dont il a besoin pour faire ses propres constructions, pour réaliser une tâche et pour aboutir à une production collective porteuse d'un modèle de connaissances original. Le formateur, pour sa part, accompagne cette démarche en offrant au groupe et à chaque apprenant son soutien et son assistance dans la réalisation de la tâche et dans la construction de son modèle de connaissances. Au terme du processus, les constructions cognitives varient d'un acteur à l'autre. Comme le montre la figure 4.2, elles sont toutes différentes; les modèles de connaissances de l'expert, de l'apprenant et du groupe ne sont pas exactement les mêmes. Chacun a élaboré une structure cognitive et une organisation de l'information qui lui est propre. 
Figure 4.2

LA DYNAMIQUE COGNITIVE DE L'APPRENTISSAGE COLLABORATIF

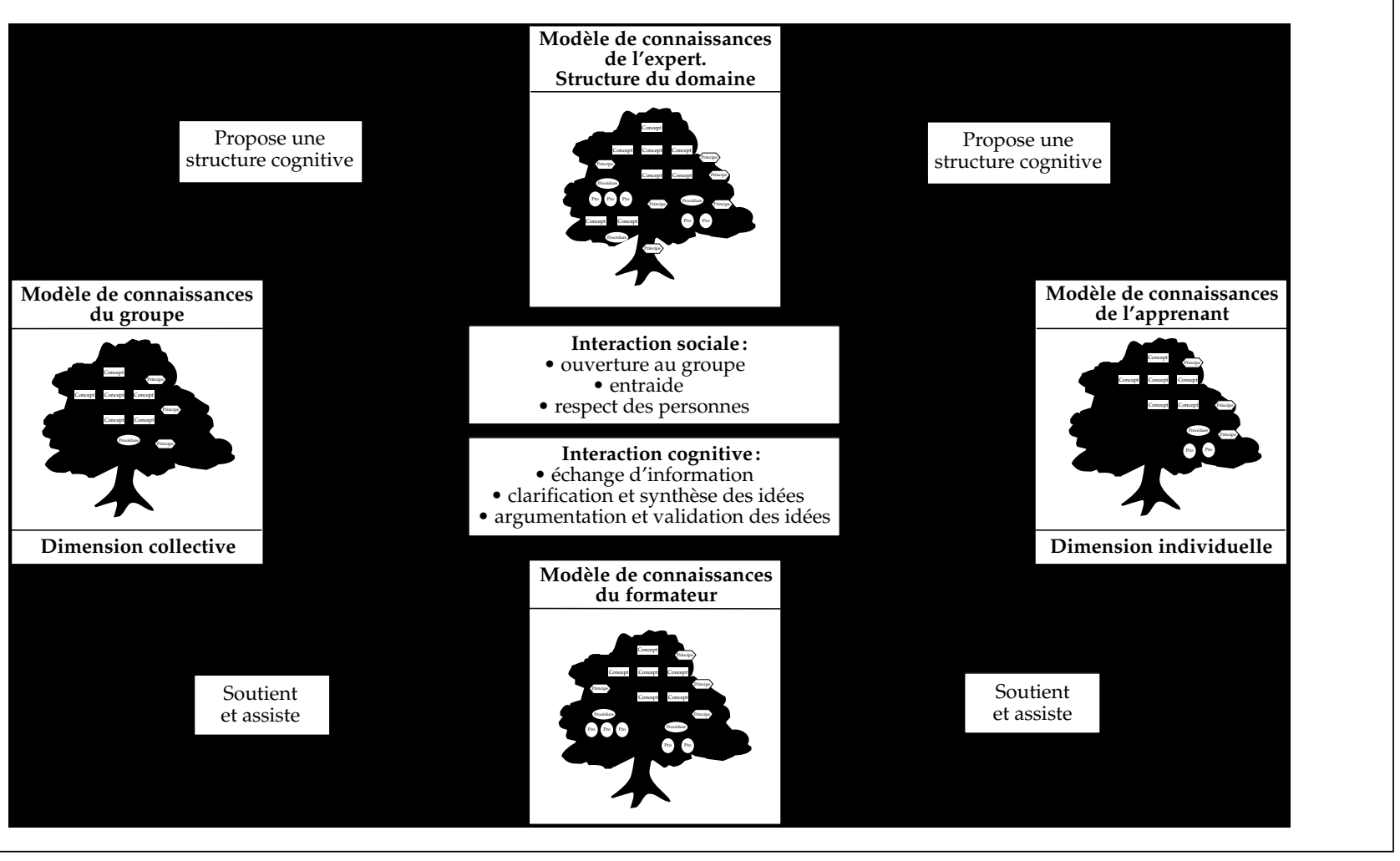




\section{LA DÉMARCHE DE COLLABORATION}

La dynamique cognitive que nous venons de décrire traduit une vision idéale que nous voulons proposer au concepteur d'environnements d'apprentissage virtuels comme guide dans l'élaboration de scénarios d'apprentissage et la conception d'activités pédagogiques. On aura compris que cette dynamique cognitive s'applique à des apprentissages qui portent sur des problèmes et des situations complexes, qui favorisent l'interaction, qui nécessitent une pensée réfléchie, qui mobilisent les ressources des apprenants et qui les amènent à se dépasser. Il n'est pas toujours facile pour le concepteur d'imaginer de telles situations d'apprentissage. Dans le but de fournir des informations qui lui faciliteront la tâche, nous poursuivons notre étude avec l'analyse de la démarche de collaboration pour montrer la dynamique cognitive à l'œuvre.

Pour baliser la démarche de collaboration, nous proposons un déroulement en phases. Nous faisons cette proposition en nous basant sur notre expérience de la collaboration en mode virtuel et après avoir étudié différentes démarches de coopération présentielles dont celle proposée par Reid, Forrestal et Cook (1989). Ces derniers ont mis au point, à l'intention des jeunes apprenants, une démarche de coopération en cinq étapes : l'engagement, l'exploration, la transformation, la présentation et la réflexion. Cette démarche ne convient pas à la collaboration entre apprenants adultes ni à l'exécution de tâches ou à la réalisation d'activités en mode virtuel. Selon notre expérience du travail asynchrone en mode virtuel, une démarche morcelée comportant autant d'étapes est laborieuse, difficile à gérer et laisse trop peu de latitude et de liberté à l'apprenant. À partir des principes de la collaboration et des principes andragogiques exposés dans les chapitres précédents, nous avons simplifié la démarche de Reid et al. en réduisant le nombre d'étapes pour en faciliter la coordination, pour lui donner plus de souplesse et laisser plus de contrôle à l'apprenant. 
Dans la démarche de collaboration, nous retrouvons trois temps forts qui n'ont pas de limites très précises dans le temps. C'est pourquoi nous parlons d'une évolution en phases qui peuvent chevaucher:

- l'exploration des connaissances et de la matière qui se traduit par la recherche et un premier traitement de l'information ;

- en même temps que se construit le modèle de connaissances de l'apprenant, la négociation des idées en vue de l'élaboration par le groupe d'un modèle de connaissances qui reflète la pluralité des points de vue;

- la réflexion personnelle et collective pour apprécier ou évaluer les modèles de connaissances, ceux des apprenants, de l'expert et du groupe, et un retour sur le processus de collaboration du groupe.

Chacune de ces phases renvoie à un processus de travail individuel et à un processus de travail de groupe qui produisent un résultat. Nous les décrivons dans les sections qui suivent et les résumons dans les tableaux 4.1 et 4.2 .

\section{L'EXPLORATION DES CONNAISSANCES}

$\mathrm{Au}$ cours de cette première phase, l'apprenant explore l'objet à connaître, décrypte les informations qui lui sont accessibles et dont on peut dire qu'elles sont l'instanciation du modèle de connaissances de l'expert. L'apprenant met ces informations en relation avec ce qu'il sait; il les appréhende avec ses structures cognitives. Ce faisant, il fait le point sur ses propres connaissances et sur leur étendue. L'exploration est une phase qui vise à cerner l'objet à étudier par la recherche d'information, par l'identification des concepts et des points de vue et constitue une première tentative pour comprendre et organiser l'information. Elle amène l'apprenant à faire un premier tri des informations qu'il juge pertinentes, délaissant ce qu'il juge inutile pour l'atteinte $\mathrm{du}$ but qu'il poursuit. Cette phase repose en bonne partie sur le travail individuel qui se déroule dans l'espace privé de l'apprenant. Elle se prolonge par un travail de groupe, dans l'espace de communication. 
Le scénario d'apprentissage prévoira des tâches ou des activités de groupe permettant aux apprenants de poser diverses questions reliées à l'objet ou au domaine d'apprentissage; de discuter des stratégies et des méthodes pour repérer, trier, ordonner et donner une première structure à l'information. Ces activités invitent les apprenants à exprimer librement leurs idées à travers, par exemple, des activités de remue-méninges, de partage d'informations, de clarification des idées, de discussion sur les stratégies de recherche et de repérage d'information, etc. Le résultat du travail, pour l'apprenant et pour le groupe, peut prendre plusieurs formes: une ébauche de carte conceptuelle ou de table des matières, un plan de travail, une série de questions ou d'énoncés, une prise de position, un point de vue, etc.

L'expérience nous a souvent permis d'observer une très forte participation des apprenants au cours de cette première phase de la collaboration. L'enthousiasme du début, le désir de connaître les autres et de se faire connaître, le besoin d'information et d'orientation de même que la nature peu structurée des activités expliquent cette intense activité.

\section{L'ÉLABORATION DU MODÈLE DE CONNAISSANCES}

Après le premier contact avec l'objet à connaître, l'apprenant, informé de la tâche à réaliser, poursuit ses recherches et ses réflexions. Il formule ses premiers énoncés, élabore ses premières propositions ou ses premiers points de vue. À cette phase, les activités sont plus ou moins structurées, conçues en fonction du degré $\mathrm{d}$ 'autonomie des apprenants. Il peut s'agir de mener une recherche documentaire, faire une analyse critique, se préparer à un débat, élaborer une prise de décision, travailler à la résolution de problème, etc. Elles exigent que chaque apprenant explicite ses idées, développe un raisonnement, bâtisse par lui-même une argumentation suffisamment étayée et qu'il en fasse part à ses pairs.

En parallèle à cette activité individuelle, l'apprenant répond et réagit aux propositions de ses pairs. Il participe activement aux travaux du groupe qui, en tant que groupe, réalise sa propre tâche, élabore sa propre construction, fait sa propre synthèse. Sous-jacent aux réalisations du groupe se trouve un modèle de connaissances autour duquel s'établit un consensus. Ce consensus 
ne doit pas être perçu comme un geste de renonciation au modèle de connaissances construit par chacun. Il doit plutôt être vu comme un signe de maturité chez le groupe et chez les participants qui ont à cœur d'atteindre l'objectif commun qu'ils se sont fixé. C'est à ce moment que l'apprenant utilise à fond les ressources de sa pensée créative pour faire sa propre synthèse, confronter son modèle au modèle collectif, le consolider et le valider. La coexistence dans l'environnement d'apprentissage de plusieurs modèles, celui de l'expert, celui du groupe et ceux des apprenants, n'est qu'une reconnaissance du fait que les connaissances ne sont pas les mêmes pour tous.

Invités à ne pas s'en remettre aveuglément à ce qui est proposé par le modèle de connaissances de l'expert (le savoir socialement constitué), à penser par eux-mêmes avec leurs pairs, à forger leur propre construction et à participer à celle du groupe, les apprenants adoptent un autre rythme d'échange dans cette phase de négociation et de validation. Ils font des interventions plus longues et mieux structurées. Les commentaires spontanés et les propos irréfléchis n'ont pas leur place. Chaque intervention exige un investissement de temps et d'énergie réel, le défi étant de transcender les positions individuelles pour atteindre l'objectif commun. Les apprenants doivent pouvoir disposer de suffisamment de temps pour donner cours à ce processus, pour s'adonner à la réflexion profonde et au développement d'une pensée complète, ce que le concepteur de l'environnement aura prévu. En outre, les interventions sécurisantes du formateur les inviteront à prendre leur temps pour apprendre, à adopter une attitude critique par rapport à la rapidité, à la compétitivité, à la recherche de la productivité et du rendement à outrance, trop souvent instituées en valeurs dans notre société qualifiée de manière antinomique de «société du savoir».

\section{L'ÉVALUATION DES APPRENTISSAGES}

\section{ET DU PROCESSUS DE COLLABORATION}

La troisième phase en est une de réflexion sur les apprentissages individuels et de groupe. Elle prend la forme d'un bilan ou d'une synthèse des événements ainsi que d'une évaluation par les apprenants de la réussite de la collaboration et de la cohésion du groupe. 
C'est l'occasion pour chacun de revoir, une dernière fois, son modèle de connaissances. Avant de clore l'apprentissage, cette réflexion sert à alimenter l'évaluation que chacun fait de son autonomie et de ses habiletés à apprendre et collaborer pour apprendre. Cette phase veut permettre aux apprenants de faire part des difficultés qu'ils ont pu éprouver au cours de la réalisation des tâches, de leurs incertitudes concernant la qualité et l'efficacité de leur processus d'apprentissage et de leur perception de la collaboration.

Souvent pressé par le temps qui manque en fin de formation, le groupe pourrait être tenté de condenser ou de télescoper cette phase. C'est pourquoi le scénario d'apprentissage doit prévoir une période de temps suffisante, clairement identifiée, pour mener cette réflexion. Le groupe doit avoir et prendre le temps de conclure et de clore l'apprentissage sans quoi les apprenants ne pourront tirer de la démarche de collaboration tous les enseignements qu'ils devraient. Ils ne pourront non plus acquérir une conscience plus aiguë des exigences que pose la collaboration et des bénéfices qu'ils en retirent.

L'exercice du bilan peut s'appuyer sur un journal de bord qu'on aura demandé au groupe et aux apprenants de tenir, sur une analyse des productions du groupe pour lesquelles on aura prévu un système d'archivage ou encore sur l'analyse du contenu des échanges qui pourrait être facilitée par l'utilisation d'un outil d'analyse automatisé.

\section{RÉSUMÉ DE LA DÉMARCHE DE COLLABORATION}

En guise de conclusion à cette section, nous résumons au tableau 4.1 la démarche de collaboration en donnant pour chacune des phases les principales caractéristiques du processus individuel et $\mathrm{du}$ processus de groupe et en indiquant les résultats. Dans le tableau 4.2, nous complétons cette synthèse en reprenant les trois phases de la collaboration, soit l'exploration, l'élaboration et l'évaluation, et en les mettant en relation avec les trois composantes de base de la collaboration, soit la communication, l'engagement et la coordination. 
Tableau 4.1

Processus et RÉSUltantes DeS TROIS PHASES DE LA DÉMARCHE COLlaborative

\begin{tabular}{|c|c|c|c|}
\hline \multirow{2}{*}{ Phases } & \multicolumn{2}{|c|}{ Processus } & \multirow{2}{*}{ Résultantes } \\
\hline & de l'apprenant & du groupe & \\
\hline Exploration & $\begin{array}{l}\text { Recherche d'information. } \\
\text { Dialogue intérieur. } \\
\text { Jugement sur la pertinence et } \\
\text { l'utilité des informations. }\end{array}$ & $\begin{array}{l}\text { Interaction avec les pairs pour aller } \\
\text { plus loin dans l'exploration de } \\
\text { l'objet à connaître. } \\
\text { Identification des informations } \\
\text { pertinentes et utiles. }\end{array}$ & $\begin{array}{l}\text { Sélection d'informations } \\
\text { pertinentes et utiles. } \\
\text { Informations plus ou moins } \\
\text { structurées. } \\
\text { Plan pour la poursuite du travail. }\end{array}$ \\
\hline $\begin{array}{l}\text { Élaboration } \\
\text { - négociation }\end{array}$ & $\begin{array}{l}\text { Organisation personnelle des idées, } \\
\text { ébauche d'un modèle. } \\
\text { Retour sur son propre modèle, } \\
\text { révision, amélioration. }\end{array}$ & $\begin{array}{l}\text { Considération des idées et des } \\
\text { points de vue de chacun. } \\
\text { Réaction aux idées des autres et } \\
\text { essai de structuration des } \\
\text { contributions multiples. } \\
\text { Proposition d'une structure. }\end{array}$ & $\begin{array}{l}\text { Successivement, à travers une } \\
\text { production collective: } \\
\text { - diverses propositions de modèle } \\
\text { de connaissances, } \\
\text { - modèle de connaissances du } \\
\text { groupe. }\end{array}$ \\
\hline - validation & $\begin{array}{l}\text { Consolidation et formalisation du } \\
\text { modèle personnel. }\end{array}$ & $\begin{array}{l}\text { Prise de position de chacun par } \\
\text { rapport à la production et au } \\
\text { modèle du groupe. } \\
\text { Conciliation, intégration des points } \\
\text { de vue. }\end{array}$ & $\begin{array}{l}\text { Consensus sur la production et le } \\
\text { modèle de connaissances du } \\
\text { groupe reflétant les visions } \\
\text { multiples. }\end{array}$ \\
\hline Évaluation & $\begin{array}{l}\text { Réflexion sur la signification } \\
\text { personnelle à donner aux } \\
\text { apprentissages. } \\
\text { Évaluation des stratégies de } \\
\text { collaboration utilisées et de la } \\
\text { contribution aux travaux du } \\
\text { groupe. }\end{array}$ & $\begin{array}{l}\text { Réflexion sur la signification } \\
\text { collective à donner aux } \\
\text { apprentissages et sur leur utilité } \\
\text { sociale. } \\
\text { Évaluation de la performance et de } \\
\text { la productivité du groupe. }\end{array}$ & $\begin{array}{l}\text { Prise de conscience de l'utilité } \\
\text { sociale des nouvelles } \\
\text { connaissances. } \\
\text { Prise de conscience des exigences } \\
\text { et des bénéfices de la collaboration. }\end{array}$ \\
\hline
\end{tabular}


Tableau 4.2

LES COMPOSANTES DU MODÈLE DE COLLABORATION

ET LES PHASES DE LA DÉMARCHE COLLABORATIVE

\begin{tabular}{|c|c|c|c|}
\hline \multirow{2}{*}{ Phases de la collaboration } & \multicolumn{3}{|c|}{ Composantes de la collaboration } \\
\hline & Communication & Engagement & Coordination \\
\hline $\begin{array}{l}\text { L'exploration } \\
\text { Phase de démarrage } \\
\text { Permet d'explorer le domaine et } \\
\text { d'établir des liens entre les } \\
\text { idées exprimées. } \\
\text { Vise surtout à faire un choix } \\
\text { critique de l'information } \\
\text { (pertinence et utilité). }\end{array}$ & $\begin{array}{l}\text { Élaboration de microstructures } \\
\text { par des activités de } \\
\text { - remue-méninges, } \\
\text { - partage d'idées, } \\
\text { - partage d'information, } \\
\text { - clarification des idées, } \\
\text { - discussion de stratégies } \\
\text { d'exploration du domaine et } \\
\text { de recherche d'information. }\end{array}$ & $\begin{array}{l}\text { Engagement social } \\
\text { - prise de conscience du but } \\
\text { commun et expression des } \\
\text { intérêts et objectifs } \\
\text { personnels, } \\
\text { - formulation des attentes de } \\
\text { chacun envers le groupe, } \\
\text { - premier stade du } \\
\text { développement d'une } \\
\text { communauté d'apprenants, } \\
\text { - prise de conscience de } \\
\text { l'importance de la } \\
\text { participation. } \\
\text { Engagement cognitif } \\
\text { - consensus sur le but, } \\
\text { - méthode de travail du } \\
\text { groupe, } \\
\text { - résultats anticipés. }\end{array}$ & $\begin{array}{l}\text { Organisation du travail } \\
\text { - reconnaissance de } \\
\text { l'architecture de } \\
\text { l'environnement virtuel } \\
\text { incluant les lieux de } \\
\text { communication, } \\
\text { - formation des équipes, } \\
\text { - établissement d'un plan de } \\
\text { travail, } \\
\text { - orientation cognitive du } \\
\text { travail, } \\
\text { - développement d'un } \\
\text { consensus sur les modalités } \\
\text { de collaboration. }\end{array}$ \\
\hline
\end{tabular}


Tableau 4.2

LES COMPOSANTES DU MODÈLE DE COLLABORATION

ET LES PHASES DE LA DÉMARCHE COLLABORATIVE (suite)

\begin{tabular}{|c|c|c|c|}
\hline \multirow{2}{*}{ Phases de la collaboration } & \multicolumn{3}{|c|}{ Composantes de la collaboration } \\
\hline & Communication & Engagement & Coordination \\
\hline $\begin{array}{l}\text { L'élaboration des } \\
\text { connaissances } \\
\text { Phase culminante } \\
\text { Permet de développer } \\
\text { l'interdépendance positive au } \\
\text { sein du groupe. } \\
\text { Vise la construction collective } \\
\text { d'un modèle de connaissances } \\
\text { par la négociation et la } \\
\text { validation. }\end{array}$ & $\begin{array}{l}\text { Participation aux échanges: } \\
\text { - prises de position, } \\
\text { - la reconnaissance et la } \\
\text { rétroaction sur les points de } \\
\text { vue des autres. } \\
\text { Élaboration de macrostructures } \\
\text { par des activités telles que: } \\
\text { - l'analyse critique, } \\
\text { - le débat, } \\
\text { - la prise de décision, } \\
\text { - la résolution de problèmes. }\end{array}$ & $\begin{array}{l}\text { Participation régulière et } \\
\text { volontaire. } \\
\text { Prise de conscience de la } \\
\text { prédominance de l'objectif } \\
\text { commun et du processus } \\
\text { collaboratif. } \\
\text { Transcendance des intérêts } \\
\text { individuels pour refléter ceux } \\
\text { du groupe. }\end{array}$ & $\begin{array}{l}\text { Choix d'une méthode } \\
\text { d'animation et d'un animateur. } \\
\text { Décisions relatives à l'archivage } \\
\text { de l'information (publique, } \\
\text { privée) et au partage des } \\
\text { ressources (degré de partage). } \\
\text { Gestion des travaux individuels } \\
\text { et de groupe et suivi de leur } \\
\text { avancement. }\end{array}$ \\
\hline $\begin{array}{l}\text { L'évaluation } \\
\text { Phase de réflexion } \\
\text { Permet à l'apprenant de } \\
\text { consolider ses connaissances, de } \\
\text { faire un retour sur le processus } \\
\text { de collaboration et sur ses } \\
\text { habiletés en la matière. } \\
\text { Vise à reconnaître le sens des } \\
\text { apprentissages sur l'objet à } \\
\text { connaître et sur la collaboration. }\end{array}$ & $\begin{array}{l}\text { Bilan de l'exercice de } \\
\text { collaboration et des } \\
\text { connaissances acquises. } \\
\text { Bilan de la qualité des } \\
\text { constructions du groupe. }\end{array}$ & $\begin{array}{l}\text { Bilan de la cohésion et de la } \\
\text { productivité du groupe. }\end{array}$ & $\begin{array}{l}\text { Évaluation du modèle et des } \\
\text { modalités de collaboration. } \\
\text { Enseignements à en tirer. }\end{array}$ \\
\hline
\end{tabular}




\section{LES HABILETÉS DE L'APPRENANT}

\section{LES HABILETÉS COGNITIVES}

À chacune des phases de la collaboration, la dynamique cognitive que l'on souhaite voir s'établir au sein du groupe sollicite de l'apprenant un ensemble d'habiletés cognitives de haut niveau que nous associons à la pensée réflexive telle que Pallascio et Lafortune (2000) la décrivent. La pensée réflexive est un construit qui «s'articule autour d'une pensée critique et créative, de compétences argumentatives et d'habiletés métacognitives» (p. 3). Pour ces auteurs, chacun de ces niveaux de pensée se détaille comme suit :

- la pensée critique permet de distinguer, dans un contexte donné, les informations les plus pertinentes au regard du but poursuivi;

- la pensée créative vise l'émergence de synthèses originales et fructueuses;

- les compétences argumentatives soutiennent l'expression de la pensée réflexive, le savoir-dire d'arguments susceptibles de la faire partager et d'en montrer la rigueur et le bien-fondé ;

- la métacognition renvoie à un ensemble de connaissances que chacun possède de son propre processus cognitif et à l'utilisation de ces connaissances pour gérer ses processus mentaux.

La pensée réflexive procure une souplesse et une liberté intellectuelle qui aide l'individu à exercer son libre arbitre et qui contribue à l'accomplissement cognitif et social. On ne peut imaginer que tous les apprenants possèdent d'emblée les habiletés de la pensée réflexive; elles sont à cultiver. Selon Daniel et al. (2000), la coopération et, selon nous, la collaboration pourraient contribuer à les développer.

Pour favoriser le développement de la pensée réflexive chez l'apprenant, il faut pouvoir la reconnaître. Selon Baffrey-Dumont (2000), elle se traduit par l'usage de jugements réflexifs que l'on peut distinguer des jugements préréflexif et quasi réflexif. Cette auteure définit ainsi ces trois types de jugements. 
1. Le jugement préréflexif s'exprime lorsque le sujet croit que la connaissance est absolument correcte et certaine.

2. Le jugement quasi réflexif est le fait des sujets qui reconnaissent que les affirmations concernant la connaissance contiennent des éléments d'incertitude.

3. Le jugement réflexif s'observe chez les sujets qui considèrent la connaissance comme provisoire et évolutive; qui sont capables de prendre en considération des cadres de référence multiples incluant leurs contradictions; qui savent intégrer les éléments du contexte pour arriver à formuler une synthèse dans une unité plus intégrée. Au moment de cette intégration, le sujet construit ses connaissances en s'engageant dans certaines valeurs, en adhérant à des croyances et en faisant place à la subjectivité.

Le formateur qui accompagne les apprenants doit pouvoir repérer la présence de ces jugements et développer des stratégies conséquentes pour favoriser ceux qui traduisent une pensée réflexive. Le concepteur qui développe l'environnement doit, de son côté, y intégrer les conditions et le soutien pour aider les apprenants à apprendre à penser. Il tiendra compte des conditions proposées par Martineau (2000) pour qui apprendre à penser:

- doit être un objectif explicite,

- doit se faire dans un contexte signifiant,

- est facilité par un contexte d'interaction sociale,

- requiert des occasions nombreuses de pratique,

- est facilité par l'entraînement à la métacognition,

- implique une préoccupation de transfert.

Baffrey-Dumont a observé que des formulations correspondant aux trois types de jugement réflexif, quasi réflexif et préréflexif peuvent coexister dans le discours d'un individu. Elle remarque toutefois que le niveau de jugement réflexif ne se manifeste que si les niveaux préréflexif et quasi réflexif ont été acquis. Il faut donc encourager ceux qui sont capables de jugement 
réflexif à faire des efforts pour toujours l'atteindre et travailler avec les autres pour qu'ils apprennent à penser et à dépasser les niveaux préréflexif et quasi réflexif.

Si un bon nombre d'éducateurs et de formateurs s'entendent pour dire qu'apprendre à penser implique le développement de compétences cognitives et métacognitives, nous voulons rapporter ici la réflexion de Romainville (2000) sur la métacognition. Cet auteur remet en question le présupposé largement partagé par les recherches et les pratiques actuelles en éducation selon lequel le développement de la métacognition constitue l'un des meilleurs moyens d'augmenter l'efficacité cognitive des apprenants. Dans son analyse critique, il observe que la réflexion sur l'acte d'apprendre peut entraver l'acte d'apprendre lui-même, le distraire. Elle peut être contre-productive. Il y a un risque de ralentir l'action ou même d'en arrêter son exécution si, en pleine réalisation de la tâche, on demande à l'apprenant de prendre conscience de son fonctionnement, de ses propres processus de pensée et de ses propres démarches pour les comprendre et les gérer. L'entraînement à la métacognition doit se faire progressivement, dans le cadre d'activités circonscrites. Ce que Romainville veut souligner, c'est le fait que la métacognition doit être sollicitée au bon moment et pour la bonne situation d'apprentissage.

En terminant cette section, nous présentons un tableau synthèse qui montre comment peuvent s'exprimer les habiletés de la pensée réflexive à travers les trois phases de la collaboration et les divers rôles que l'on peut proposer aux apprenants pour en favoriser le développement. Aux quatre grandes habiletés de Pallascio et Lafortune (2000), nous avons ajouté l'habileté à écrire le discours qui se rapporte à la pensée critique et créative, à l'argumentation et à la métacognition. C'est une habileté transversale qui nous apparaît importante et qu'il ne faut pas négliger puisque les apprenants qui évoluent dans des environnements virtuels s'adonnent à une communication essentiellement textuelle. Dans ce contexte, l'acquisition du «savoir-dire » par l'écriture nous apparaît fondamentale pour que la collaboration soit efficace. 
Tableau 4.3

LES HABILETÉS DE LA PENSÉE RÉFLEXIVE SOLLICITÉES AUX TROIS PHASES DE LA COLLABORATION

\begin{tabular}{|c|c|c|c|c|}
\hline \multirow{2}{*}{$\begin{array}{l}\text { Phases de la } \\
\text { collaboration }\end{array}$} & \multicolumn{4}{|c|}{ Habileté de mise en texte dans les forums de... } \\
\hline & la pensée critique & la pensée créative & l'argumentation & la métacognition \\
\hline $\begin{array}{l}\text { L'exploration } \\
\text { - préparation } \\
\text { Rôles de l'apprenant: } \\
\text { - chercheur, } \\
\text { - informateur, } \\
\text { - concepteur d'idées } \\
\text { nouvelles. }\end{array}$ & $\begin{array}{l}\text { Rechercher de } \\
\text { l'information. } \\
\text { Identifier et comprendre } \\
\text { les idées et les concepts. } \\
\text { Choisir l'information } \\
\text { pertinente. }\end{array}$ & $\begin{array}{l}\text { Établir des liens entre } \\
\text { des idées et des points } \\
\text { de vue multiples. } \\
\text { Faire de nouveaux liens } \\
\text { entre les idées et les } \\
\text { concepts exprimés. }\end{array}$ & & $\begin{array}{l}\text { S'informer pour } \\
\text { améliorer ses stratégies } \\
\text { de recherche et de } \\
\text { traitement de } \\
\text { l'information. } \\
\text { Rechercher des méthodes } \\
\text { de travail plus efficaces. } \\
\text { Se donner un cadre de } \\
\text { travail adéquat. } \\
\text { Préparer un plan. }\end{array}$ \\
\hline $\begin{array}{l}\text { L'élaboration } \\
\text { - négociation } \\
\text { Rôles de l'apprenant: } \\
\text { - ingénieur cognitif, } \\
\text { - présentateur, } \\
\text { - débatteur, } \\
\text { - avocat, } \\
\text { - arbitre. }\end{array}$ & $\begin{array}{l}\text { Extraire la structure et } \\
\text { les principes du } \\
\text { domaine. } \\
\text { Débattre des points de } \\
\text { vue opposés. } \\
\text { Comparer, faire la } \\
\text { critique et évaluer des } \\
\text { opinions. } \\
\text { Porter un jugement sur } \\
\text { la qualité et la pertinence } \\
\text { des connaissances. }\end{array}$ & $\begin{array}{l}\text { Reconstruire en } \\
\text { proposant une nouvelle } \\
\text { intégration de } \\
\text { l'information. } \\
\text { Faire des concessions. } \\
\text { Concilier les divergences } \\
\text { pour atteindre un } \\
\text { consensus. }\end{array}$ & $\begin{array}{l}\text { Exposer son point de } \\
\text { vue en faisant des } \\
\text { démonstrations } \\
\text { probantes. } \\
\text { Réfuter des arguments. } \\
\text { Reconnaître la justesse } \\
\text { de l'argumentation. }\end{array}$ & $\begin{array}{l}\text { S'interroger sur ses } \\
\text { propres démarches et sur } \\
\text { celles du groupe. } \\
\text { Reconnaître et respecter } \\
\text { les rythmes et les } \\
\text { différences de chacun. }\end{array}$ \\
\hline
\end{tabular}


Tableau 4.3

LES HABILETÉS DE LA PENSÉE RÉFLEXIVE SOLLICITÉES AUX TROIS PHASES DE LA COLLABORATION (suite)

\begin{tabular}{|c|c|c|c|c|}
\hline \multirow{2}{*}{$\begin{array}{l}\text { Phases de la } \\
\text { collaboration }\end{array}$} & \multicolumn{4}{|c|}{ Habileté de mise en texte dans les forums de... } \\
\hline & la pensée critique & la pensée créative & l'argumentation & la métacognition \\
\hline $\begin{array}{l}\text { - validation } \\
\text { Rôles de l'apprenant: } \\
\text { - analyste, } \\
\text { - décideur. }\end{array}$ & $\begin{array}{l}\text { Accepter des } \\
\text { propositions jugées } \\
\text { valables. }\end{array}$ & & & $\begin{array}{l}\text { Amorce de transfert: } \\
\text { prise de conscience des } \\
\text { similitudes et des } \\
\text { différences et façons } \\
\text { d'adapter les } \\
\text { connaissances à d'autres } \\
\text { contextes. }\end{array}$ \\
\hline $\begin{array}{l}\text { L'évaluation } \\
\text { - réflexion } \\
\text { Rôles de l'apprenant: } \\
\text { - philosophe, } \\
\text { - juge. }\end{array}$ & $\begin{array}{l}\text { Retracer les événements } \\
\text { de l'expérience } \\
\text { d'apprentissage. } \\
\text { Faire la comparaison et } \\
\text { la critique constructive } \\
\text { du modèle de } \\
\text { connaissances du } \\
\text { groupe et de ceux des } \\
\text { apprenants. }\end{array}$ & $\begin{array}{l}\text { Faire une synthèse de } \\
\text { l'ensemble de } \\
\text { l'expérience en } \\
\text { formulant une } \\
\text { appréciation de la } \\
\text { cohésion et de la } \\
\text { productivité du groupe. }\end{array}$ & $\begin{array}{l}\text { Savoir faire un bilan } \\
\text { des connaissances et } \\
\text { des processus de } \\
\text { collaboration. }\end{array}$ & $\begin{array}{l}\text { Faire un bilan de ses } \\
\text { propres positions ou de } \\
\text { ses points de vue. } \\
\text { Juger sa propre } \\
\text { contribution au travail } \\
\text { du groupe. } \\
\text { Évaluer ses compétences } \\
\text { comme apprenant et } \\
\text { comme collaborateur. }\end{array}$ \\
\hline
\end{tabular}




\section{APPRENDRE À COLLABORER}

Si l'on peut dire que la collaboration est une situation propice pour apprendre à penser, il ne faut pas perdre de vue que la collaboration, surtout en mode virtuel, est, elle aussi, un exercice qui s'apprend. Savoir collaborer n'est pas inné : cela s'apprend, au même titre que des connaissances ou des compétences disciplinaires. Savoir collaborer requiert des habiletés cognitives et métacognitives ainsi que des compétences sociales, des attitudes psychologiques et des dispositions affectives. Pour collaborer, les apprenants doivent être prêts à le faire. Ils doivent être disposés à s'engager, capables d'assumer la responsabilité de leur apprentissage, ouverts aux autres et motivés à participer activement aux travaux du groupe. S'ils ne le sont pas, il faut les y préparer. À cet égard, le concepteur de l'environnement d'apprentissage et le formateur ont chacun des rôles et des responsabilités spécifiques. Le concepteur doit proposer au formateur des stratégies et des démarches d'interventions qui auront comme but explicite le développement des habiletés de collaboration. Le formateur doit savoir faire un diagnostic de ces habiletés, choisir parmi les démarches d'intervention qui lui sont proposées celles qui sont les plus appropriées et, au besoin, savoir les adapter.

Collaborer n'est pas un exercice abstrait, c'est un geste concret. Apprendre à collaborer ne doit donc pas se limiter à savoir ce qu'il faut faire sans pouvoir le faire effectivement. La conceptualisation de la collaboration doit être mobilisée dans l'action et cette action doit être efficace. Comme le propose LundgrenCayrol (1996), l'apprentissage de la collaboration, surtout avec des apprenants qui en sont à leurs premières expériences, passe d'abord par la négociation en groupe du modèle et des modalités de collaboration qui s'appliqueront pour réaliser l'activité de formation. Cette activité de préparation amène le groupe à se donner une cible commune, à s'engager dans l'atteinte de son but et à développer une perception positive de la collaboration. Elle responsabilise les apprenants et les amène à préciser le rôle qu'ils peuvent et qu'ils veulent jouer, à prendre conscience du degré de leur engagement et à établir les règles de participation en des termes concrets: rythme de travail, contribution aux forums de discussion, couverture du contenu à apprendre, soutien apporté aux autres, etc. 


\section{Négociation du modèle de collaboration par le groupe}

Le forum de télégestion est le lieu tout indiqué pour négocier le modèle de collaboration qui régira l'activité du groupe. Cette activité doit être guidée et rigoureusement encadrée par le formateur surtout si le groupe en est à ses premières expériences de collaboration en milieu virtuel. Il s'agit principalement pour le groupe de découvrir, de comprendre et d'évaluer ses besoins pour ensuite définir la manière dont fonctionnera la collaboration et dont les tâches seront accomplies. Cet exercice n'a rien de théorique; il permet de traduire, en termes pratiques, les attentes et les perceptions et d'établir un consensus sur ce que les apprenants vont faire dans et avec le groupe. Le modèle de collaboration négocié par le groupe au début de la formation peut constamment être revu et renégocié.

Dès le démarrage de la formation, le formateur explique au groupe, de manière explicite et concrète, le fonctionnement qu'il entrevoit et les exigences de la démarche d'apprentissage qui leur est proposée. Ensuite, le groupe analyse le modèle de collaboration proposé et en évalue la pertinence par rapport à ses besoins et aux avantages qu'il en tire. Une discussion conduit à une prise de décision collective sur les règles qui régiront l'activité individuelle et celle du groupe. La collaboration doit être perçue de manière positive par les membres du groupe et apporter une plus-value à l'apprentissage dans lequel ils vont s'engager. Dans cette optique, ils négocieront entre eux et avec le formateur les modalités de la collaboration : participation (rythme, fréquence, ampleur des interventions), constitution d'équipes, nature des contributions de chacun, paramètres ou caractéristiques des productions du groupe ou des équipes, échéancier de travail et tout autre aménagement qui convient. Il devrait résulter de ce travail amorcé dès le début de la formation une représentation commune et partagée de la collaboration, un accord sur ce que chacun est prêt à donner et sur ce qu'il retirera. Toutefois, les modalités et les règles de collaboration pourront être remises en question en tout temps au cours du déroulement de l'activité de formation. Le modèle pourra être modifié pour, par exemple, aider le groupe à être plus efficace, favoriser une meilleure cohésion, faire mieux correspondre le modèle à ce que les membres attendent de la collaboration, etc. Le tableau 4.4 résume le processus continu de la négociation. 
Tableau 4.4

Processus CONTINU DE NÉGOCIATION DU MODÈle DE COLLAbORATION

\begin{tabular}{|c|c|c|c|}
\hline Étapes de la formation & Apprenant & Groupe & Formateur \\
\hline $\begin{array}{l}\text { Démarrage de la } \\
\text { formation } \\
\text { But: s'entendre sur un } \\
\text { modèle de } \\
\text { collaboration. }\end{array}$ & $\begin{array}{l}\text { Exprime ses perceptions, } \\
\text { ses attentes et ses besoins } \\
\text { au regard de la } \\
\text { collaboration. } \\
\text { Indique la contribution et } \\
\text { l'effort de collaboration } \\
\text { qu'il est prêt à fournir. }\end{array}$ & $\begin{array}{l}\text { Discute le modèle proposé } \\
\text { en fonction de ses attentes, } \\
\text { de ses besoins et de ses } \\
\text { capacités. } \\
\text { Au besoin, négocie un } \\
\text { nouveau modèle de } \\
\text { collaboration ou des } \\
\text { modalités mieux adaptées. }\end{array}$ & $\begin{array}{l}\text { Analyse de manière pragmatique le modèle } \\
\text { de collaboration proposé: } \\
\text { - contenu et rythme de la communication, } \\
\text { - engagement envers le groupe pour l'atteinte } \\
\text { de l'objectif commun, } \\
\text { - coordination de la tâche à réaliser, } \\
\text { - calendrier des travaux, } \\
\text { - rôles à jouer, } \\
\text { - habiletés à manifester, } \\
\text { - ressources à utiliser, } \\
\text { - etc. } \\
\text { S'assure que les apprenants ont une } \\
\text { représentation commune et consensuelle de la } \\
\text { collaboration qui va s'établir. }\end{array}$ \\
\hline $\begin{array}{l}\text { En cours de formation } \\
\text { But: optimiser la } \\
\text { collaboration. }\end{array}$ & $\begin{array}{l}\text { Exprime ses satisfactions et } \\
\text { insatisfactions au regard de } \\
\text { la collaboration. }\end{array}$ & $\begin{array}{l}\text { Apprécie sa productivité. } \\
\text { Au besoin, propose des } \\
\text { ajustements au modèle de } \\
\text { collaboration. }\end{array}$ & $\begin{array}{l}\text { Fait une évaluation formative du } \\
\text { fonctionnement de la collaboration. }\end{array}$ \\
\hline $\begin{array}{l}\text { Au terme de la } \\
\text { formation } \\
\text { But: prendre } \\
\text { conscience que la } \\
\text { collaboration est } \\
\text { une expérience } \\
\text { d'apprentissage. }\end{array}$ & $\begin{array}{l}\text { Fait un retour sur la } \\
\text { démarche de collaboration. } \\
\text { Identifie les habiletés } \\
\text { acquises et celles qui restent } \\
\text { à acquérir en matière de } \\
\text { collaboration. }\end{array}$ & $\begin{array}{l}\text { Fait un retour sur la } \\
\text { démarche de collaboration. } \\
\text { Apprécie la productivité du } \\
\text { groupe et les résultats } \\
\text { obtenus. }\end{array}$ & $\begin{array}{l}\text { Évalue la collaboration en rapport avec son } \\
\text { objet, son but et son fonctionnement. }\end{array}$ \\
\hline
\end{tabular}


En plus d'utiliser des activités formelles pour préparer et mettre en place la collaboration, l'apprentissage de la collaboration se fait par l'observation du formateur qui doit donner l'exemple par ses actes et son discours. Il démontrera l'habileté au partage et la capacité d'ouverture aux autres et aux idées des autres, l'habileté à négocier et la capacité de concilier sa pensée avec celle des autres. Le formateur transmettra également le message que le leadership est indispensable à la collaboration. Pour développer le leadership, Tagg (1994) propose de confier progressivement aux apprenants la responsabilité d'animer leurs propres échanges d'animateur. Le rôle du formateur consistera alors à simplement les soutenir dans leur animation. Les apprenants seront ainsi amenés à prendre des décisions, à exercer de plus en plus de contrôle sur leur apprentissage et à gagner en autonomie (Lundgren-Cayrol, 1996). La figure 4.3 présente la courbe idéale de croissance du contrôle exercé par l'apprenant sur son apprentissage et du gain d'autonomie tout au long de la démarche de formation.

Figure 4.3

L'ÉVOLUTION DE L'APPRENANT

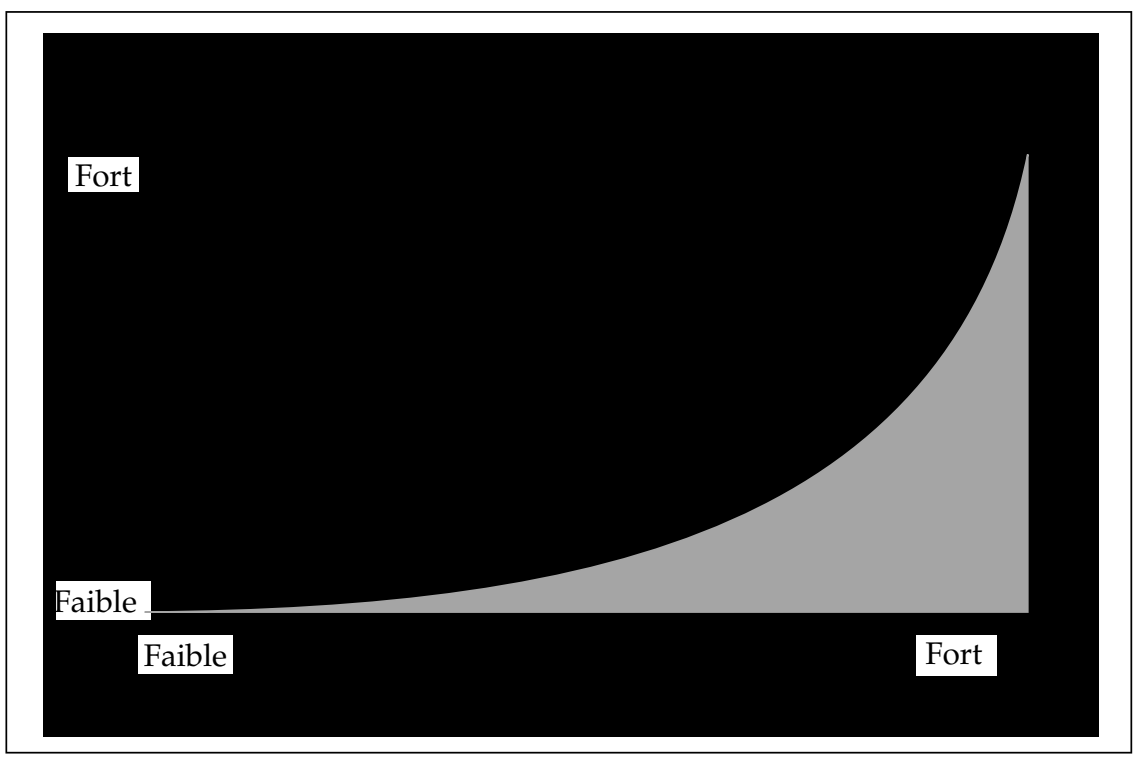




\section{LE SOUTIEN À LA CONSTRUCTION DES CONNAISSANCES}

Dans la démarche collaborative, le formateur agit en soutien à l'apprentissage. C'est un accompagnateur dont le rôle évolue au fur et à mesure que les apprenants acquièrent plus d'autonomie et qu'ils prennent plus de responsabilité à l'égard de leur apprentissage.

\section{LES RÔLES DU FORMATEUR}

Plusieurs auteurs ont décrit les nombreuses facettes du rôle du formateur en formation à distance (Henri, 1988 ; Feenberg, 1989 ; Damphousse, 1996 ; Paquin et al., 1996). C'est lui qui conseille, guide, aide à comprendre le contenu de l'apprentissage, organise et anime les groupes et les équipes et évalue les travaux des apprenants. Il intervient sur une base individuelle auprès des apprenants pour clarifier le contenu d'apprentissage, pour leur proposer des méthodes de travail intellectuel et pour leur suggérer l'application de stratégies efficaces. Son rôle est essentiellement de faciliter l'acquisition des connaissances (Feenberg, 1989).

Dans les environnements virtuels de collaboration, le formateur se manifeste et intervient surtout dans les forums de discussion. Tout comme dans une situation de collaboration présentielle, il répond aux besoins du groupe, anime et alimente les échanges, favorise la participation de chacun, stimule les interactions et veille au déroulement harmonieux des travaux. Sa mission est d'aider le groupe à progresser vers l'atteinte de l'objectif commun de la manière la plus efficace possible.

Ricciardi Rigault et al. (1993) et Damphousse (1996) ont montré que, dans le cadre des forums de discussion, le formateur ne doit pas tenir plusieurs rôles à la fois. Dans les lieux de télédiscussion, il est modérateur de débats et peut y traiter de l'objet d'apprentissage comme le ferait un expert de contenu. Dans les lieux de télétravail, le formateur aide les apprenants à prendre en charge leur animation et à gérer leurs travaux d'équipe; il joue alors le rôle de «coach». Dans la télégestion, il devient gestionnaire pédagogique et voit à l'organisation de l'apprentissage. D'un lieu à 
l'autre, son rôle se distingue. En exerçant ces divers rôles et tout au long de la collaboration, il sera appelé à moduler ses interventions selon la progression du groupe.

\section{LES INTERVENTIONS SPÉCIFIQUES}

\section{AUX DIFFÉRENTES PHASES DE LA COLLABORATION}

À chaque phase de la collaboration, les interventions du formateur se modifient au rythme de l'évolution des apprenants euxmêmes qui, graduellement, deviennent de plus en plus autonomes et de plus en plus habiles à collaborer. À la phase d'exploration, le formateur est un modérateur, son encadrement est plus étroit. À la phase d'élaboration des connaissances, il intervient comme facilitateur. Finalement, il agira comme simple animateur

\section{Tableau 4.5}

\section{LES RÔLES ET LES INTERVENTIONS DU FORMATEUR POUR LE SOUTIEN À L'ACQUISITION DES CONNAISSANCES}

\begin{tabular}{|c|c|}
\hline $\begin{array}{l}\text { Interventions aux } \\
\text { trois phases }\end{array}$ & Exemples d'intervention \\
\hline $\begin{array}{l}\text { Modérateur } \\
\text { Phase d'exploration }\end{array}$ & $\begin{array}{l}\text { - Présenter l'objet à connaître (structure et modèle de } \\
\text { connaissances), suggérer des stratégies cognitives pour la } \\
\text { recherche d'information, proposer des méthodes de } \\
\text { travail. } \\
\text { - Établir les liens entre les idées et les concepts. } \\
\text { - Voir à l'établissement des règles de collaboration. }\end{array}$ \\
\hline $\begin{array}{l}\text { Facilitateur } \\
\text { Phase d'élaboration }\end{array}$ & $\begin{array}{l}\text { - Soutenir et encourager l'élaboration des connaissances, } \\
\text { apporter des clarifications sur l'objet de connaissance, } \\
\text { suggérer des cheminements pour apprendre et travailler. } \\
\text { - Assister le groupe dans la négociation et la validation } \\
\text { des connaissances. }\end{array}$ \\
\hline $\begin{array}{l}\text { Animateur } \\
\text { Phase d'évaluation }\end{array}$ & $\begin{array}{l}\text { - Intervenir sur la demande des apprenants, attitude } \\
\text { réactive. } \\
\text { - Au besoin, stimuler la réflexion et la rétroaction pour en } \\
\text { améliorer la compréhension. } \\
\text { - Évaluer les acquis. }\end{array}$ \\
\hline $\begin{array}{l}\text { Évaluateur } \\
\text { A toutes les phases }\end{array}$ & $\begin{array}{l}\text { - Évaluer le fonctionnement du groupe (climat, cohésion, } \\
\text { productivité) et planifier des interventions en } \\
\text { conséquence. } \\
\text { - Préciser les critères d'évaluation des apprentissages et le } \\
\text { mode de notation. } \\
\text { - Évaluer les productions cognitives. }\end{array}$ \\
\hline
\end{tabular}


Figure 4.4

LE FORMATEUR: DE MODÉRATEUR À ANIMATEUR

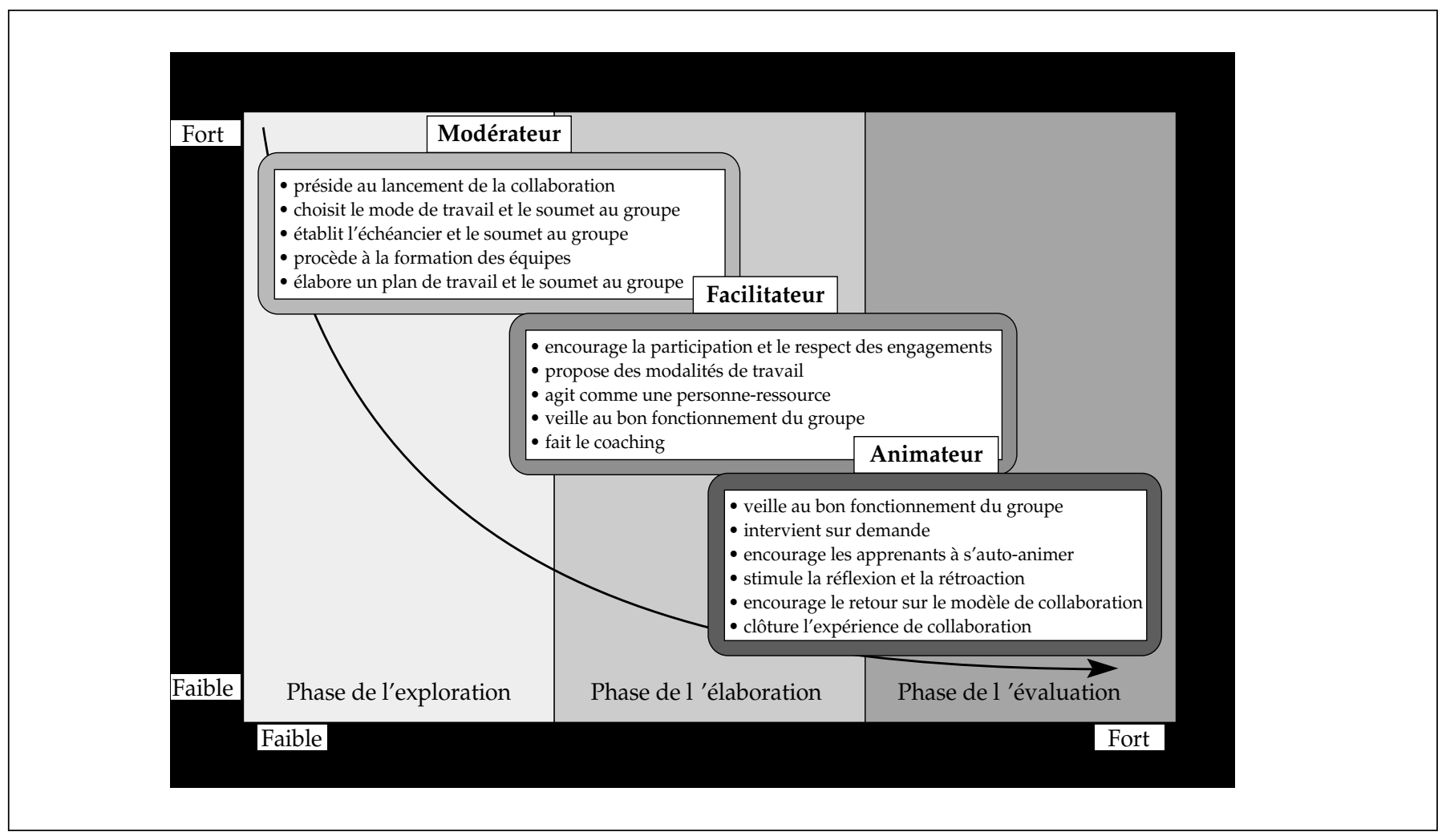


à la phase d'évaluation. Toutefois, à chacune des trois phases, il conservera un rôle d'évaluateur, constamment préoccupé de faire le diagnostic de la situation, d'évaluer ce qui se passe et la façon dont cela se passe afin d'intervenir adéquatement en véritable accompagnateur.

L'évolution du rôle du formateur au cours des trois phases de la démarche collaborative veut permettre à l'apprenant d'exercer un plus grand contrôle, de développer sa responsabilité et son autonomie à l'égard de son apprentissage. Celui-ci s'efface graduellement pour ne devenir qu'un simple animateur des échanges (Brown et Palincsar, 1987; Lave et Wenger, 1991). La figure 4.4 montre comment ses interventions se modulent d'une phase à l'autre.

\section{L'ÉVALUATION DES APPRENTISSAGES}

Au cours d'une formation à distance en mode virtuel, les apprentissages sont nombreux et de plusieurs ordres: apprentissages cognitifs, psychosociaux, méthodologiques et technologiques. Dans la plupart des cas, tous ces apprentissages n'ont pas à être évalués. Généralement, on cherche surtout à mesurer les apprentissages cognitifs. Mais dans l'approche collaborative, l'évaluation des apprentissages doit également porter sur le processus de collaboration.

\section{L'ÉVALUATION DES APPRENTISSAGES COGNITIFS}

La liberté dont dispose l'apprenant dans la démarche de collaboration et la personnalisation de son modèle de connaissances pose de sérieux problèmes aux évaluateurs lorsqu'ils doivent attester qu'au terme d'une formation les apprenants possèdent bel et bien telles ou telles compétences. Il n'existe pas de solutions toutes faites au problème de l'évaluation et du contrôle de la qualité des apprentissages. Jonassen (1995) nous ouvre cependant des pistes intéressantes par ses travaux sur l'évaluation des modèles mentaux. 


\section{Comprendre et évaluer les modèles mentaux des apprenants}

Nous avons mentionné au début de ce travail que la démarche collaborative en est une qui sert mieux que toute autre la construction de connaissances complexes, dites avancées, par le transfert de connaissances et par la résolution de situations ou de problèmes complexes. La résolution de problèmes est une compétence hautement valorisée dans la société; elle est l'apanage de la pensée de l'homme. C'est pourquoi Jonassen propose d'axer la formation sur la résolution de problèmes qui sont qualifiés de complexes parce qu'ils exigeront la mise en application non pas de un, mais de plusieurs systèmes de connaissances. Mais au terme d'une formation, comment savoir si l'apprenant a acquis suffisamment de connaissances pour résoudre des problèmes complexes réels? Comment évaluer ses apprentissages? Une des façons de procéder consisterait à évaluer les représentations mentales que l'apprenant utilise pour résoudre, dans leur contexte, des problèmes peu ou mal structurés.

Pour savoir si les connaissances construites par l'apprenant sont complètes et s'il peut les transférer, il faut d'abord comprendre le modèle mental qu'il a élaboré, puis l'évaluer. La performance d'un apprenant peut être expliquée, à tout le moins en partie, par la qualité des modèles mentaux qu'il possède et qu'il sait appliquer pour résoudre des problèmes (Gott, Benett et Gillet, 1988, cités par Jonassen, 1995).

\section{Qu'est-ce qu'un modèle mental et comment l'évaluer?}

La théorie des modèles mentaux tente d'expliquer le phénomène de la compréhension chez l'humain. Cette compréhension se traduit par des modèles qui fournissent des représentations qui, à leur tour, fournissent des moyens d'interpréter, de mémoriser et de communiquer l'information, et de contrôler ses performances (Wild, 1996). Les modèles mentaux correspondent aux conceptions que chacun élabore dans son univers mental sous forme de représentations d'objets ou d'événements et de relations structurelles entre ces objets ou ces événements. Les modèles mentaux se composent donc d'objets et de relations. Ces objets sont des concepts (des nœuds) et leurs relations sont des liens (des verbes) 
qui énoncent la nature des liens entre les objets (Jonassen, 1995). Il en résulte un réseau ou une carte de relations qui décrit le domaine de connaissances représenté par le modèle mental. Les modèles mentaux décrivent comment chacun conçoit un domaine. Ils varient d'une personne à l'autre en raison des connaissances antérieures, des habiletés individuelles, des croyances et des valeurs. Construits à l'aide de raisonnement analogique et métaphorique, on peut les décrire ainsi :

- ce sont des représentations internes que chacun élabore;

- le langage est la clé pour comprendre les modèles mentaux ; ils sont l'objet d'une médiatisation linguistique ;

- ils peuvent être représentés par des réseaux de concepts ;

- le sens donné aux concepts est compris dans leurs relations avec les autres concepts ;

- le sens social donné aux concepts découle de l'intersection des différents modèles mentaux que l'on trouve en chacun (Carley et Palmquist, cités par Jonassen, 1995).

Jonassen ajoute que les modèles mentaux sont des constructions dynamiques, multimodales et multidimensionnelles. Ils sont complexes et épistémiques, c'est-à-dire qu'ils sont un moyen $\mathrm{d}^{\prime}$ exprimer comment nous savons ce que nous savons. Ils ne sont pas facilement compris des autres et c'est pourquoi ils doivent être inférés par la manifestation d'une performance.

La performance qui traduit un modèle mental est faite de plusieurs types de connaissances qui, elles, peuvent être évaluées. Jonassen retient:

- les connaissances structurales: connaissances de la structure d'un domaine ;

- les connaissances procédurales de performance: capacité de résoudre un problème ;

- les connaissances procédurales de réflexion: capacité d'enseigner à un autre comment faire ;

- l'image du système: capacité de développer des images mentales ;

- la métaphore: capacité de relier le système étudié à un système existant ; 
Tableau 4.6

\section{CRITÈrES POUR ÉVALUER LES MODÈlES MENTAUX}

\begin{tabular}{|l|l|}
\hline Critères d'évaluation & Stratégie d'évaluation \\
\hline 1. Cohérence & Penser tout haut (pour les connaissances structurales) \\
\hline 2. Pertinence personnelle & Compte rendu cognitif, interview cognitif \\
\hline 3. Intégration & Simulation cognitive \\
\hline 4. Fidélité avec la réalité & Comparaison avec le modèle de l'expert \\
\hline 5. Imagerie & Génération de métaphores et d'analogies \\
\hline 6. Complexité & Penser tout haut (pour les connaissances structurales) \\
\hline 7. Applicabilité, transférabilité & Enseigner à un autre, penser tout haut \\
\hline 8. Inférence & Utilisation du bon modèle au bon moment \\
\hline
\end{tabular}

Source: Adapté de Jonassen, 1995.

- la connaissance exécutive : capacité d'utiliser le bon modèle mental au bon moment (un problème complexe exige le recours à plusieurs modèles mentaux).

Les modèles mentaux peuvent être évalués au moyen de huit critères: cohérence, pertinence, intégration personnelle, fidélité avec la réalité, imagerie, complexité, transférabilité, inférence, en utilisant des moyens basés sur la verbalisation. Nous résumons, au tableau 4.6, le modèle d'évaluation développé par Jonassen.

\section{L'ÉVALUATION DE LA DÉMARCHE DE COLLABORATION}

L'évaluation des apprentissages dans la perspective cognitiviste est une procédure complexe. Les tests de mémorisation souvent utilisés en éducation ne fournissent pas la mesure des apprentissages dans toute leur complexité. Dans la perspective de la démarche de collaboration, l'évaluation des apprentissages englobe les acquis cognitifs et les capacités à collaborer. Les premiers pourront être évalués, comme le propose Jonassen, par l'analyse des productions des apprenants et les seconds le seront par l'observation des comportements et l'analyse des perceptions. Trois formes d'évaluation peuvent être utilisées, l'évaluation-diagnostic, l'évaluation formative et l'évaluation sommative, aux différentes phases (voir tableau 4.7). Nous les résumons en nous appuyant sur les définitions fournies par Legendre (1993). 
Tableau 4.7

\section{LES CRITÈRES D'ÉVALUATION}

\begin{tabular}{|c|c|c|}
\hline $\begin{array}{l}\text { Évaluation aux trois phases de la } \\
\text { collaboration }\end{array}$ & $\begin{array}{l}\text { Critères d'évaluation cognitive à partir des } \\
\text { productions de l'apprenant }\end{array}$ & $\begin{array}{l}\text { Critères d'évaluation des capacités de } \\
\text { collaboration }\end{array}$ \\
\hline $\begin{array}{l}\text { Phase d'exploration } \\
\text { Évaluation-diagnostic } \\
\text { But: apprécier les caractéristiques } \\
\text { individuelles qui pourront avoir des } \\
\text { influences positives ou négatives sur } \\
\text { l'apprentissage. }\end{array}$ & $\begin{array}{l}\text { - Rapport au modèle de connaissances } \\
\text { de l'expert } \\
\text { - Habiletés cognitives }\end{array}$ & $\begin{array}{l}\text { Mesures prises au tout début: } \\
\text { - degré d'autonomie, } \\
\text { - habiletés sociales, } \\
\text { - nombre d'expériences antérieures de } \\
\text { collaboration, } \\
\text { - perceptions de la collaboration, } \\
\text { - compétences méthodologiques, } \\
\text { - compétences technologiques reliées à } \\
\text { l'utilisation de l'environnement } \\
\text { d'apprentissage. }\end{array}$ \\
\hline $\begin{array}{l}\text { Phases d'élaboration et d'évaluation } \\
\text { Évaluation formative } \\
\text { But: informer l'apprenant des objectifs } \\
\text { atteints ou non en vue de lui assurer le } \\
\text { soutien nécessaire. }\end{array}$ & $\begin{array}{l}\text { - Les concepts } \\
\text { - La structure et les liens entre les } \\
\text { concepts } \\
\text { - La clarté de la représentation } \\
\text { - La pertinence }\end{array}$ & $\begin{array}{l}\text { Inventaire des perceptions relatives à la } \\
\text { productivité du groupe: } \\
\text { - l'usage de temps, } \\
\text { - le développement des idées, } \\
\text { - l'habileté à prendre des décisions, } \\
\text { - les comportements du groupe, } \\
\text { - l'accomplissement du groupe. }\end{array}$ \\
\hline $\begin{array}{l}\text { Phase d'évaluation } \\
\text { Évaluation sommative } \\
\text { But: porter un jugement sur le degré de } \\
\text { réalisation des apprentissages en vue de } \\
\text { décider de la promotion, de l'accréditation } \\
\text { ou du décernement d'un diplôme. }\end{array}$ & $\begin{array}{l}\text { - L'applicabilité } \\
\text { - La transférabilité }\end{array}$ & $\begin{array}{l}\text { Participation notée en fonction de: } \\
\text { - la régularité des contributions, } \\
\text { - le respect du contrat et de l'échéancier } \\
\text { tels qu'ils ont été négociés et consentis } \\
\text { par le groupe/équipe. }\end{array}$ \\
\hline
\end{tabular}


L'évaluation-diagnostic s'applique surtout au début de la collaboration, avant que les apprenants ne commencent la démarche. Elle porte sur les connaissances reliées à l'objet d'apprentissage, sur les compétences et les connaissances se rapportant au travail collaboratif et sur les compétences techniques reliées à l'utilisation de l'environnement d'apprentissage. Les résultats sont utilisés pour aider le formateur à estimer si les apprenants sont prêts à s'engager et s'ils ont les connaissances, les capacités ou les aptitudes requises pour collaborer. Ils lui permettent d'anticiper les difficultés de cheminement et, le cas échéant, de proposer des mesures pour les surmonter.

L'évaluation formative survient surtout à la deuxième et la troisième phase de la collaboration. Elle a pour but d'informer de façon continue sur les difficultés éprouvées et les progrès réalisés. Elle est surtout orientée vers une aide à l'apprenant et au groupe, mais elle peut aussi être utilisée comme élément de motivation extrinsèque pour l'apprenant et comme un indice de productivité pour le groupe. L'évaluation formative peut être faite par le formateur ou par les pairs. L'évaluation sommative intervient à la troisième phase, en fin de formation; elle est assurée par le formateur qui porte un jugement sur le degré de réalisation des apprentissages. En plus d'utiliser les productions des apprenants comme témoins des apprentissages, l'évaluation est basée sur le bilan établi par les apprenants pour clore la collaboration. 


\section{Conclusion}

ans nos vies personnelle et professionnelle, nous nous prêtons à la collaboration sans toujours en être conscients. Nous manifestons notre engagement en posant des gestes qui concourent à l'atteinte d'un objectif que nous partageons avec d'autres. Certaines personnes sont plus portées que d'autres à collaborer. La collaboration, pour elles, relève d'une philosophie ou d'un style de vie qui les amènent à intégrer avec aisance les interactions sociales et cognitives de même que tout ce qui découle de l'appartenance à un groupe. D'emblée, elles voient un sens dans l'engagement et dans la collaboration. Elles y trouvent un accomplissement, de la satisfaction et de la gratification. Par ailleurs, $\mathrm{d}$ 'autres personnes perçoivent les démarches de groupe, les interactions et les négociations inhérentes à la collaboration comme contraignantes, comme des obstacles à leur cheminement. Elles hésitent à prendre part à des collaborations et peuvent même refuser systématiquement d'y souscrire.

La disposition à collaborer varie d'une personne à l'autre en fonction du profil de personnalité, des valeurs et des expériences antérieures. S'ajoutent aussi d'autres facteurs immédiats tels que le contexte, le but poursuivi, les actions en cause, les enjeux, etc. $\mathrm{Au}$ sein des groupes de formation, comme dans les autres milieux, on retrouve chez les apprenants des attitudes variées à l'égard de la collaboration. On se demande alors comment traiter cette diversité. Mais avant de répondre à cette question, on doit statuer sur l'expérience de collaboration. Il faut savoir si elle doit faire partie de la formation, pourquoi et dans quel but. 
La formation ne vise pas essentiellement à préparer des travailleurs pour répondre aux besoins du marché. Sa finalité est plutôt de former des citoyens qui, ensemble, peuvent contribuer à l'épanouissement de la société. C'est au nom de valeurs sociales et de la croissance personnelle que les éducateurs et les formateurs valorisent l'apprentissage de la collaboration et la collaboration dans l'apprentissage. Adopter la collaboration comme but et comme moyen de formation, cela ne veut pas dire opter pour la facilité. C'est un choix exigeant et ceux qui s'y engagent méritent toute notre admiration. Comme nous avons pu le voir au cours de cette étude, la démarche collaborative exige un certain dépassement autant de la part de l'apprenant que du formateur. Elle invite à une mobilisation authentique et à une ouverture aux autres; elle est faite de concessions et d'un certain renoncement pour atteindre le but.

Les situations de collaboration peuvent être vécues avec difficulté, voire avec irritation par les apprenants selon qu'elles sont plus ou moins bien préparées, expliquées et intégrées dans la formation, selon le temps et les ressources dont on dispose, selon le désir de chacun de se dépasser en relevant le défi de collaborer pour apprendre. Plusieurs conditions doivent donc être réunies pour que la collaboration soit possible. Nous avons tenté de les identifier et de les décrire en espérant faciliter la pratique collaborative et en favoriser la réussite. Le défi qui nous semble le plus important à relever, c'est d'arriver à concevoir et animer la démarche de collaboration avec le souci du respect de chacun et la recherche $\mathrm{du}$ bien de tous. Ce paradoxe existera toujours dans la collaboration qui le reconnaît en proposant une structure souple et adaptable. Contrairement à la coopération qui propose un structure prédéfinie, la collaboration, par une approche négociée, permet à chacun de mesurer, de définir et de communiquer son engagement. Chacun est appelé à dire jusqu'où il veut ou il peut s'engager. Pour gérer une telle situation, il importe que le groupe puisse négocier des modalités de collaboration pour créer une situation viable et optimale.

La collaboration est de plus en plus présente dans les formations. Des programmes entiers de formation universitaire procédant essentiellement par la résolution de problèmes ou la 
réalisation de projets y accordent une place très importante ${ }^{1}$. Le passage d'une logique d'enseignement à une logique d'apprentissage a, dans tous les cas, nécessité une profonde réflexion qui a suscité l'émergence d'un nouvel esprit de formation. On s'est interrogé sur ce qu'est la formation ; pour répondre à cette question, il a fallu repenser l'apprentissage. Actuellement, la volonté d'intégrer l'usage des nouvelles technologies dans la formation, qu'il s'agisse de formation en entreprise ou universitaire, devrait s'accompagner d'une redéfinition de la formation et de l'apprentissage. Cette redéfinition devrait par le fait même mener à une réflexion sur les potentialités et les limites des technologies en fonction des finalités poursuivies et de la conception nouvelle de l'apprentissage qu'on aura développée.

Nous avons voulu alimenter cette réflexion en associant notamment l'approche constructiviste à la démarche de collaboration. La pédagogie découlant de cette association est ancrée dans le milieu et exploite des scénarios pédagogiques basés sur des situations et des problématiques réelles. Ces scénarios, procédant selon une démarche d'exploration, d'élaboration et de validation des connaissances, encadrent l'apprentissage collaboratif que nous avons décrit comme un processus conversationnel où les pairs jouent un rôle important aux côtés du formateur et de l'expert-contenu. Nous avons également proposé le recours aux technologies non pas uniquement pour permettre aux apprenants de réaliser à distance ce processus, mais parce que ces nouveaux outils peuvent représenter un soutien efficace à la collaboration (communication, engagement et coordination). L'usage des technologies que nous proposons nous permet d'affirmer notre conception de l'apprentissage.

Nous sommes conscientes que le recours à de nouveaux moyens technologiques ne conduit pas automatiquement à l'innovation pédagogique. Trop souvent, les schèmes hérités se glissent de manière insidieuse dans ce que nous croyons être «nouveau » et nous nous rendons compte que, sous un appareil

1. Par exemple, au Québec, le programme de médecine de l’Université de Sherbrooke. 
considéré avant-gardiste et à la fine pointe, les anciens paradigmes s'appliquent et les mêmes structures se camouflent. Sachant que des changements véritables en pédagogie demeurent difficiles à implanter, nous souhaitons que le présent travail pourra contribuer à un nouvel examen de l'acte pédagogique et à la découverte du potentiel des technologies pour l'améliorer. Nous souhaitons également que ceux et celles qui entreprendront cet exercice veillent à éviter le piège des changements superficiels qui masquent les vieux modèles en leur donnant une apparence de renouveau. 


\section{Bibliographie}

ABrami, P., Chambers, P., POUlsen, C., De Simone, C., D'APOllonia, S. et HOWDEN, J. (1995). Classroom connections: Understanding and using cooperative learning. Toronto, HarcourtBrace \& Company.

ACKERMAN, M.S. (1996). «Providing social interaction in the digital library». Dans CSCW '96 Conference on Computer Supported Work, Association for Computing Machinery.

ADAMS, D.M. et HAMM, M.E. (1990). Cooperative learning : Critical thinking and collaborations across the curriculum. Springfield, Ill., C.C. Thomas.

Anderson, J.R., Corbett, A.T., Koedigner, K.R. et Pelletier, R. (1995). "Cognitive tutors: Lessons learned». The Journal of Learning Sciences, vol. 4, no 2, p. 167-207.

APPELT, W. (1995). Interoperable tools for cooperation support using the World-Wide-Web. German National Research Centre for Information Technology Institute for Applied Information Technology. <http://orgwis.gmd.de/COOPWWW/ERCIMWS.html>

BAFFREY-DUMONT, V. (2000). «Pensée postformelle, jugement réflexif et pensée réflexive». Dans Pallascio, R. et Lafortune, L. (dir.), Pour une pensée réflexive en éducation. Sainte-Foy (Québec), Presses de l'Université du Québec, p. 9-29.

BALES, R.F. (1950). Interaction process analysis: A method for the study of small groups. Cambridge, Ma, Addison-Wesley.

BEDER, H.W. et DARKENWALD, G.G. (1982). «Differences between teaching adults and preadults. Some propositions and findings ». Adult Educational Journal, vol. 32, p. 142-155. 
BÉLANGER, P.C., avec la collaboration de P. Ross (1997). " Vers une pédagogie de l'hyper-savoir? ». Revue de l'éducation à distance, vol. $12, \mathrm{n}^{\text {os }} 1$ et 2 , p. 29-48.

BERGE, Z. (1995). «Facilitating computer conferencing: Recommendations from the field». Educational Technology, vol. 35, no 1, p. 22-30.

Bernard, M. (1999). Penser la mise à distance en formation. Paris, Montréal, L'Harmattan.

BERTRAND, Y. (1990). Théories contemporaines de l'éducation. Montréal, Les éditions Agence d'arc inc.

BODER, A. (1993). «Le juste-à-temps de la connaissance dans les organisations ». Dans Actes du $4^{e}$ Colloque international en informatique cognitive des organisations (ICO '93). Montréal, 4 au 7 mai.

BoHliN, R.M., MiLHEIM, W.D. et VIECHNICKI, K. (1994). "The development of a model for the design of motivational adult instruction in higher education ». Journal of Educational Technology systems, vol. 22, no 1, p. 3-17.

BONK, C.J. et KING, K.S. (1995). Computer conferencing and collaborative writing tools: Starting a dialogue about student dialogue. <http://www-csc195.indiana.edu/csc195/bonk.html>

BoOMER, G. (1990). «Empowering the learner». Dans Brubacher, M., Payne, R. et Rickett, K. (dir.), Perspectives on small group learning. Oakville, Ontario, Rubicon Press.

BORDELEAU, Pierre (1994). Apprendre dans des environnements pédagogiques informatisés. Montréal (Québec), Les Éditions Logiques.

BOUTON, C. et GARTH, R.Y. (1983). "Students in learning groups. Active learning through conversations ". Dans Bouton, C. et Garth, R.Y. (dir.), Learning in groups : New directions in teaching and learning. San Francisco, Jossey-Bass.

BRIGGS, L.J. (1981). Instructional design: Principles and applications. Engelwood Cliffs, N.J., Educational Technology Publications. BROOKFIELD, S. (1986). Understanding and facilitating adult learning. San Francisco, Jossey-Bass Publishers. 
BROWN, A.L. et PALINCSAR, A.S. (1987). "Guided, cooperative learning and individual knowledge acquisition ». Dans Resnick, L.B. (dir.), Cognition and instruction: Issues and agendas. Hillsdale, N.J., Erlbaum, p. 391-451.

Bruce, M.C., Kreeft Peyton, J. et BAtSON, T. (1993). Networkbased classrooms. Promises and realities. Cambridge, Cambridge University Press.

BURGE, E. (1994). «Learning in computer conference contexts: The learner's perspective». Journal of Distance Education, vol. 9, no 1, p. 19-43.

BURGE, E. et ROBERTS, J.M. (1993). Classrooms with a difference: A practical guide to the use of conferencing technologies. Toronto, The Ontario Institute for Studies in Education, Distance Learning Office.

CAvalier, J.C., Klein, J.D. et CAVAlier, F.J. (1995). «Effects of cooperative learning on performance, attitude, and group behaviors in a technical team environment ». ETRED, vol. 43, no 3, p. 61-71.

CERRATTO, T. et BELISLE, C. (1995). Reframing learning in CSCL Environments. <http://www-csc195.indiana.edu/csc195/cerratto.html>

CHAMBERS, M. (1999). "The efficacy and ethics of using digital multimedia for educational purposes ». Dans Tait, A. et Mills, R. (dir.), The convergence of distance and conventional education. Patterns of flexibility for individual learner. Londres et New York, Routledge.

CHAN, T.-W. et CHOU, C.-Y. (1995). Simulating a learning companion in reciprocal tutoring systems. <http://www-csc195.indiana.edu/csc195/chan.html>

CHOI, J.-I. et HANNAFIN, M. (1995). «Situated cognition and learning environments: Roles, structures, and implications for design ». ETRED, vol. 43, no 2, p. 53-69.

Cognition and Technology Group at Vanderbilt (1990). « Anchored instruction and its relationship to situated cognition ». Educational Researcher, vol. 19, no 6, p. 2-10.

Cognition and Technology Group at Vanderbilt (1991). «Technology and the design of generative learning environments ». Educational Technology, vol. 31, no 5. 
Cole, M. (1993). «Conclusions ». Dans Salomon, G. (dir.), Distributed cognitions: Psychological and educational considerations. Cambridge, Cambridge University Press.

COlEMAN, D. et FureY, D. (1996). Collaborative infrastructures for knowledge management. <http://www.collaborate.com/tip.html>

Collings, P. et WALKER, D. (1995). «Applications to support student group work». Dans CSCL '95 Proceedings, Faculty of Information Sciences and Engineering, University of Canberra, Australie, octobre.

Collins, A., Brown, J.S., et Newman, S.E. (1989). "Cognitive apprenticeship: Teaching the crafts of reading, writing, and mathematics ». Dans Resnick, L.B. (dir.), Knowing, learning, and instruction: Essays in honour of Robert Glaser, Hillsdale, N.J., Lawrence Erlbaum Associates, Inc., p. 453-494.

CRANTON, P. (1994). "Self-directed and transformative instructional development ». Journal of Higher Education, vol. 65, $\mathrm{n}^{\circ} 6$, p. 726-744.

Cross, P. (1981). Adult as learners. San Francisco, Jossey-Bass Publishers.

CROTTY, T. (1994). «Integrating distance learning activities to enhance teaching and learning ». Dans Distance learning research conference proceedings, Texas A \& M University, p. 31-37.

CROTTY, T. (1995). " Constructivist theory unites distance learning and teacher education ». DEOSNEWS, vol. 5, n 6, 612 lignes.

DAMPHOUSSE, L. (1996). Participation et animation: Un modèle d'analyse de la téléconférence assistée par ordinateur à la Téléuniversité. Mémoire de maîtrise en communication, Université du Québec à Montréal.

Daniel, M.-F., Lafortune, L., PAllascio, R. et Scleifer, M. (2000). "Communauté de recherche philosophique». Dans Pallascio, R. et Lafortune, L. (dir.), Pour une pensée réflexive en éducation. Sainte-Foy (Québec), Presses de l’Université du Québec, p. 157-180.

DAVIE, L.E. et WelLS, R. (1991). «Empowering the learner through computer-mediated communication ». The American Journal of Distance Education, vol. 5, no 1, p. 15-23. 
De la Teja, I., Lundgren-Cayrol, K. et Paquin, C. (1997). Rapport de mise à l'essai du Prototype global. Montréal, Centre de recherche LICEF, Télé-université.

DESCHÊNES, A.J. (1995). «Vers un modèle constructiviste de la production de texte ». Dans Boyer, J.Y., Dionne, J.P., Raymond, P. (dir.), La production de textes. Montréal, Les Éditions Logiques.

Development. $2^{\mathrm{e}}$ édition. New York, Prentice Hall.

DICK, W. et CAREY, L. (1985). The systematic design of instruction. Glenview, Ill., Scott, Foresman and Company.

DillenbOURG, P. (2000). Virtual Learning Environments. <http://tecfa.unige.ch/tecfa/publicat/dil-papers-2/Dil.7.5.18.pdf>

DiMOCK, H.G. (1985). How to analyze and evaluate group growth. $2^{\mathrm{e}}$ éd., Guelph, University of Guelph.

DIMOCK, H.G. (1987). Designing and facilitating training programs. 4 éd., Guelph, University of Guelph.

DONOVAN, D.C. (1995). "Computer mediated communication and the basic speech course». Interpersonal Computing and Technology: An Electronic Journal for the 21st Century, vol. 3, no 3, p. 32-53.

DORÉ, S. et BASQUE, J. (1998). «Le concept d'environnement d'apprentissage informatisé ». Revue de l'éducation à distance, vol. $13, \mathrm{n}^{\circ} 1$.

DUNING, B.S. (1993). Reaching learners through telecommunications: Management and leadership strategies for higher education. San Francisco, Jossey-Bass Publishers.

EALES, J.R.T. et WELSH, J. (1996). Design for collaborative learnability. Australie, Department of Computer Science, University of Queensland and CRC for Distributed Systems Technology.

Edelson, D.C., O'NeILL, D.K., BOMEZ, L.M. et D'AMICO, L. (1996). A design for effective support of inquiry and collaboration. School of Education and social Policy, Northwestern University, USA. $<$ http://www-csc195.indiana.edu/cscl95/edelson.html>

EHRLICH, K. et CASH, D. (1996). Turning information into knowledge: Information finding as a collaborative activity. <http://www-csc195/indiana.edu/csc195.html> 
ERICSSON, K.A et SMITH, J. (1991). Toward a general theory of expertise: Prospects and limits. Cambridge, Cambridge Universtiy Press.

FEENBERG, A. (1989). "The written world». Mindweave, Communication, Computers and Distance Education. Dans Robin Mason et Anthony Kaye (dir.), Oxford, U.K., Pergamon Press.

FESTINGER, L. (1950). "Informal social communication ». Psychological Review, vol. 57, p. 271-282.

FESTINGER, L. (1957). A theory of cognitive dissonance. Evanston, Illinois : Row, Peterson.

FISHER, G. (1995). Distributed cognition, learning webs, and domainoriented design environments.

<http://www-csc195.indiana.edu/csc195/fisher.html>

FRAASE, M. (1991). Groupware for the Macintosh. A complete guide to collaborative computing. Homewood, Ill., Richard D. Irwin, Inc.

GAGNÉ, R.M. (1985). The conditions of learning. $4^{\mathrm{e}}$ édition, New York, Holt Rinehart and Winston.

GARRISON, R.D. (1995). "Constructivism and the role of selfinstructional course materials : A reply». Distance Education, vol. 16, no 1, p. 136-140.

GuNAWARDENA, C.N. (1991a). "Current trends in the use of communication technologies for delivering distance education ». International Journal of Instructional Media, vol. 18, n 3, p. 201213.

GunAWARDENA, C.N. (1991b). «Changing faculty roles for audiographics and online teaching ». The American Journal of Distance Education, vol. 6, no 3, p. 58-70.

Gutwin, C., STARK, G. et GREENBERG, S. (1995). Support for workspace awareness in educational groupware. <http://www-csc195. indiana.edu/cscl95/gutwin.html>

GuZdial, M., TuRns, J., RAPPIN, N. et CARlson, D. (1995). Collaborative support for learning in complex domains. <http:/www-cscl95/indiana.edu/cscl95/guzdial.html>

Harasim, L. (1990). Online education. Perspectives on a new environment. New York, Prager. 
HARRI-AUGSTEIN, S. et THOMAS, L. (1991). Learning conversations. The self-organized learning way to personal and organizational growth. New York, Routledge.

Hastie, R. et Pennington, N. (1993). «Cognitive and social processes in decision making ». Dans Salomon, G. (dir.), Distributed cognitions: Psychological and educational considerations. Cambridge, Cambridge University Press.

HATANO, G. et INAGAKI, K. (1991). "Sharing cognition through collective comprehension activity ». Dans Resnick, L.B., Levine, J.M. et Teasly, S.D., Perspectives on socially shared cognition. Washington, D.C., American Psychological Association.

HILL, F. (1969). Learning thru discussion. California : Sage Publications.

HILTZ, S.R. (1987). Evaluating the virtual classroom. CCCC Technical Report 87-1, Computerized Conferencing and Communications Center, New Jersey Institute of Technology, Newark, N.J., 07102.

HILTZ, S.R. et TUROFF, M. (1982). The network nation. Human communication via computer. $4^{\mathrm{e}}$ éd., Reading, Mass., AddisonWesley Publishers Co.

HoAdleY, C.M. et HSI, S. (1993). «A multimedia interface for knowledge building and collaborative learning ». Proceedings of InterCHI'93, joint conference of ACM SIG-CHI and INTERACT, Amsterdam, the Netherlands, 24-29 avril 1993. ACM Press, p. 103-104.

HOLOYAK, K.J. (1991). «Symbolic connectionism: Toward thirdgeneration theories of expertise». Dans Ericsson, K.A. et Smith, J., Toward a general theory of expertise: Prospects and limits. Cambridge, Cambridge Universtiy Press.

HotTe, R. (1995). L'intervention tutorale dans un système d'encadrement pédagogique assisté par ordinateur (EPAO). Québec, Téléuniversité.

JONASSEN, D.H. (1995). Operationalizing mental models: Strategies for assessing mental models to support meaningful learning and design-supportive learning environments. <http://www-csc195/ indiana.edu/csc195/jonassen.html> 
JonASSEN, D., DAVIDSON, M., COLINS, M., CAMPBELL, J. et BANNAN HAAG, B. (1995). "Constructivism and computer-mediated communication in distance education ». The American Journal of Distance Education, vol. 9, no 2, p. 7-27.

KAGAN, S. (1992). Cooperative learning. San Juan Capistrano, Cal., Ressources for Teachers.

KANSElAAR, G. et ERKENS, G. (1996). A cooperative system for collaborative problem solving. Netherlands, Department of Educational Sciences, University of Utrecht.

KLEMM, W.R. et SNELL, J.R. (1996). Enriching computer-mediated group learning by coupling constructivism with collaborative learning. <http://www.usq.edu.au/electpub/e-jist/homepage.htm>

KNOWLes, M. (1990). L'apprenant adulte. Paris, Les Éditions d'Organisation.

LAURILlARD, D. (1995). "Multimedia and the changing experience of the learner ». British Journal of Educational Technology, vol. 26, no 3, p.179-189.

LAVE, J. (1993). "Situating learning in communities of practice». Dans Salomon, Gavriel (dir.), Distributed cognitions: Psychological and educational considerations. Cambridge, Cambridge University Press.

LAVE, J. et WENGER, E. (1991). Situated learning: Legitimate peripheral participation. Cambridge, Cambridge University Press.

LEBOW, D. (1991). "Constructivist values for instructional systems design: Five principles toward a new mindset». ETRED, vol. 41, no 3, p. 4-16.

LEGENDRe, R. (1993). Dictionnaire actuel de l'éducation. 2e éd., Paris, Montréal, Larousse.

LEMKE, J.L. (1994). «Discourse, dynamics, and social change». Dans Halliday, M.A.K. (dir.), Dynamics. Dynamics paper, Disponible par FTP : cynyvm.cuny.edu

LEWIN, I. (1948). «Resolving social conflicts ». Dans Lewin, Gertrude W. (dir.), Selected papers on group dynamics. New York, Harper \& Row.

LEWIS, R. (1996a). «Editorial: Cooperation or collaboration?» Journal of Computer Assisted Learning, vol. 12, n 2, p. 96. 
LEWIS, R. (1996b). "Editorial: Collaborative learning at a distance». Journal of Computer Assisted Learning, vol. 12, $\mathrm{n}^{\circ} 4$, p. 193.

LI, T. et JONASSEN, D. (1996). The effect of lesson Structures on predication and inference. Document ERIC: ED397813.

LINARD, M. (1995). «New debates on learning support». Journal of Computer Assisted Learning, vol. 11, no 4. p. 239-253.

LUNDGREN-CAYROL, K. (1996). Computer-Conferencing : A collaborative learning environment for distance education students. Thèse de doctorat, Montréal, Université Concordia.

Mager, R.F. (1962). Preparing instructional objectives. Palo Alto, Cal., Fearon.

MARTINEAU, R. (2000). "La pensée historique... une alternative réflexive précieuse pour l'éducation du citoyen ». Dans Pallascio, R. et Lafortune, L. (dir.), Pour une pensée réflexive en éducation. Sainte-Foy (Québec), Presses de l'Université du Québec, p. 208-309.

MASON, R. (1993). Computer conferencing: The last word... U.K., Beach Holme Publishers.

MASON, R. (1998). Globalising Education. Trends and applications. Londres et New York, Routledge.

MilleR, Gary E. (1995). "The undergraduate curriculum and distance education ». New Directions for Adult and Continuing Education, no 67 , p. 41-49.

MORLAN, John E. (1973). Classroom learning centers : Planning, organization, materials, and activities. Belmont, Cal., Lake Pub Co.

Mullen, B. et COPPER, C. (1994). "The relation between group cohesiveness and performance: An integration ». Psychological Bulletin, vol. 115, no 2, p. 210-227.

Pallascio, R. et LAfortune, L., dir. (2000). Pour une pensée réflexive en éducation. Sainte-Foy (Québec), Presses de 1'Université du Québec.

PAQUETTE, G. (à paraître). L'ingénierie du téléapprentissage. La conception des systèmes de connaissances distribués. 
PAQUIN, C. (1993). Analyse exploratoire pour l'utilisation de la technologie du référentiel dans un environnement de développement de matériel de formation. Mémoire de maîtrise en informatique de gestion. Montréal, Université du Québec à Montréal.

Paquin, C., Paquette, G., Bourdeau, J. et Rigault, C. (1996). Spécification des besoins. Architecture du Campus Virtuel. Rapport interne du projet HyperGuide et Recto. Montréal, Centre de recherche LICEF.

PEA, R.D. (1993). "Practices of distributed intelligence and designs for education». Dans Salomon, G. (dir.), Distributed cognitions: Psychological and educational considerations. Cambridge, Cambridge University Press.

PRADL, G.M. (1991). "Collaborative learning and mature dependency». Dans Brubacher, M., Payne, R. et Rickett, K. (dir), Perspectives on small group learning. Oakville (Ontario), Rubicon Press.

PRESCOTT, S. (1994). "What works. Building effective collaborative learning experiences ». Dans A National conference on collaborative learning. The National Center on Post-secondary Teaching, Learning and Assessment, in cooperation with Pennstate University, Continuing and Distance Education.

PUGH, S.L. (1993). "Using case studies and collaborative computer-assisted communication to support conceptual learning in a teacher-education course on critical reading ». Educational Technology, novembre, p. 30-38.

REID, J-A., FORRESTAL, P. et COOK, J. (1989). Small group learning in the classroom. Toronto, Irwin Publications.

RESNICK, L.B. (1987). Education and learning to think. Washington, D.C., Academy Press.

RicCIARDi RigAUlt, C. et al. (1993). Apprentissage collaboratif et téléconférence intelligemment assistée. Rapport de recherche présenté au CRIM. Montréal, Centre de recherche LICEF, Télé-université.

RICCIARDI RigAULT, C. (1995). «Espace virtuel pédagogique et analyse de la communication par téléconférence ». DELTA Final Conference on the Concerted Action Program: Multimedia and Distance Learning for Science and Technology. 
RicCiARDI RigAulT, C. et HENRI, F. (1989). "Support à l'apprentissage». Actes du colloque "Le transfert des connaissances en sciences et techniques ». Université de Montpellier II.

ROMAINVILLE, M. (2000). "Savoir comment apprendre suffit-il à mieux apprendre? Métacognition et améliorations des performances ». Dans Pallascio, R. et Lafortune, L. (dir.), Pour une pensée réflexive en éducation. Sainte-Foy (Québec), Presses de l'Université du Québec, p. 69-86.

ROMISZOWSKI, A.J. (1980), Designing Instructional Systems, Londres, Kogan Page.

RÖSCHEISEN, M., MOGENSEN, C. et WinOGRAD, T. (1996). Beyond browsing: Shared comments, SOAPs (Seals Of APproval), trails, and on-line communities.

<http://www-diglib. stanford.edu/rmr/TR/TR.html>

ROWNTREE, D. (1995). «Teaching and learning on-line: A correspondence education for the $21^{\text {st }}$ century? » British Journal of Educational Technology, vol. 26, no 3 .

SALOMON, G. (1992). "What does the design of effective CSCL require and how do we study its effects? »SIGCUE Outlook, vol. 21, no 3, p. 62-68.

SALOMON, G., dir. (1993). Distributed cognitions: Psychological and educational considerations. Cambridge, Cambridge University Press.

SAlOMON, G. et PERKINS, D. (1998). «Individual and social aspects of learning ». Review of Research in Education, vol. 23. <http://construct.haifa.ac.il/ gsalomon/indsoc.htm>

SCHÖN, D.A. (1994). Le praticien réflexif. Montréal, Les Éditions Logiques.

SCHRAGE, M. (1990). Shared minds. The new technologies of collaboration. New York, Random House.

SCHROEDER, E.E. et KENNY, R.F. (1995). «Learning strategies for interactive multimedia instruction: Applying linear and spatial notetaking ». Canadian Journal of Educational Communication, vol. 24, $\mathrm{n}^{\mathrm{o}} 1$, p. 27-47.

SHAw, M.E. (1981). Group dynamics. USA, McGraw Hill Book Company. 
SHEDLETSKY, L. (1993). «Minding computer-mediated communication: CMC as experiential learning ». Educational Technology, vol. 33, no 10, p. 5-10.

SMith, P.L. et RaGAN, T.J. (1992). Instructional Design. New York, Macmillan Publishing Company.

SPIRO, R.J., FELTOVICH, P.J., JACOBSON, M.J. et COUlSON, R.L. (1991). "Cognitive flexibility, constructivism, and hypertext: Random access instruction for advanced knowledge acquisition ». Dans Duffy, T. et Jonassen, D. (dir.), Constructivism and the Technology of Instruction, vol. III-Structured Domains. Hillsdale, N.J., Lawrence Erlbaum Associates Publishers, p. 57-75.

SPUCK, D.W., PrATER, D.L. et PAluMBO, D.B. (1995). «Using electronic meeting system support in the design of the graduate core curriculum ». ETRED, vol. 43, no 2, p. 71-80.

ST-ARnaud, Y. (1989). Les petits groupes. Participation et communication. Montréal, Presses de l'Université de Montréal et Les Éditions du CMI.

SteEPles, C., GOOdyear, P. et Mellar, H. (1994). «Flexible learning in higher education: The use of computer-mediated communications ». Computers in Education, vol. 22, $\mathrm{n}^{\text {os }} 1-2, \mathrm{p} .83-90$.

SUTHERS, D. et WEINER, A. (1995). Groupware for developing critical discussion skills.

<http://www-csc195/indiana.edu/csc195/ suthers.html>

TAGG, A.C. (1994). «Leadership from within: Student moderation of computer conferences". The American Journal of Distance Education, vol. 8, no 3, p. 40-50.

TARDIF, J. (1998). Intégrer les nouvelles technologies de l'information Quel cadre pédagogique? Paris, ESF.

TENNYSON, R.D., ELMORE, R.L. et SNYDER, L. (1990). «Advancements in instructional design theory: Contextual module analysis and integrated instructional strategies ». Educational Technology Research E Development, vol. 40, no 2, p. 9-22.

TOMLINSON, H. et HendersON, W. (1995). «Computer supported collaborative learning in schools: A distributed approach». British Journal of Educational Technology, vol. 26, n² 2, p. 131-140. 
TrOlliP, Stanley R. et AlESSI, Stephen M. (1991). Computer-Based Instruction: Methods and development, $2^{\mathrm{e}}$ éd., Englewood Cliffs, N.J., Prentice-Hall.

VON GLASERFELD, E. (1990). "An exposition of constructivism: Why some like it radical». Dans Klir, George J., Facets of System Science. New York, Plenum Press, p. 229-238.

VON GLASERFELD, E. (1995). Radical constructivism: A way of knowing and learning. Washington D.C., The Falmer Press.

VERDUIN, J.R. et CLARK, T.A. (1991). «Distance education». Dans Knox, A.B. (dir.), Foundations of effective practice. San Francisco, Jossey-Bass Publishers.

WELLS, R. (1995). «Size of CMC classes ». Dans First Thread-IUCCMC. On-line conference. 3-21 avril.

WiLD, M. (1996). "Mental models and computer modelling». Journal of Computer Assisted Learning, vol. 12, p. 10-21.

Wilson, Brent et Cole, Peggy (1991). «Cognitive apprenticeships: An instructional design review». Dans Proceedings of Selected Research Presentations at the Annual Convention of the Association for Educational Communications and Technology.

WiLSON, B.G. (1992). The post-modern paradigm. Constructivism and instructional design: Some personal reflections. gopher:// ccnucd.cudenver.edu/00/UCD/dept/edu/IT/wilson / postmodern.txt

WINN, W. (1990). "Some implications of cognitive theory for instructional design». Instructional Sciences, vol. 19, p. 53-69.

YELON, S. (1996). Powerful principles of instruction. New York, Longman Publishers. 



\section{Index des auteurs cités}

\begin{tabular}{|c|c|c|}
\hline A & Cavalier, F.J., 31 & $\mathbf{E}$ \\
\hline Abrami, P., 30, 32, 35, & Cavalier, J.C., 31 & Eales, J.R.T., 69 \\
\hline $112,115,118,119,120$ & Cerratto, T., 71 & Edelson, D.C., 69 \\
\hline 121,124 & Chambers, M., 5 & Ehrlich, K., 75 \\
\hline Ackerman, M.S., 111 & Chan, T.-W., 133 & Elmore, R.L., 81 \\
\hline Adams, D.M., 31 & Choi, J.-I., 133 & Ericsson, K.A., 15 \\
\hline Alessi, S.M., 50 & Chou, C.-Y., 133 & Erkens, G., 76, 86 \\
\hline Anderson, J.R., 15 & Clark, T.A., 27 & \\
\hline Appelt, W., 133 & Cognition and & $\mathbf{F}$ \\
\hline B & $\begin{array}{l}\text { lechnology Group at } \\
\text { Vanderbilt, } 17\end{array}$ & Feenberg, A., 80, 81, 125, \\
\hline $\begin{array}{l}\text { Baffrey-Dumont, V., 147, } \\
\quad 148\end{array}$ & $\begin{array}{l}\text { Cole, M., 31, 32, } 61 \\
\text { Cole, P., } 17\end{array}$ & $\begin{array}{l}156 \\
\text { Festinger, L., 83, } 97\end{array}$ \\
\hline Bales, R.F., 97 & Coleman, D., 75 & Fisher, G., 18, 61 \\
\hline Basque, J., 48, 49 & Collings, P., 119, 121 & $\begin{array}{l}\text { Forrestal, 1., } 139 \\
\text { Fraase M } 65\end{array}$ \\
\hline Beder, H.W., 27 & Collins, A., 18 & $\begin{array}{l}\text { Fraase, M., } 65 \\
\text { Furey D. } 75\end{array}$ \\
\hline Bélanger, P.C., 1, 2 & Cook, J., 139 & Furey, D., 75 \\
\hline Belisle, C., 71 & Copper, C., 83, 120, 121 & \\
\hline Berge, Z., 80, 121, 133 & Cranton, P., 18 & C \\
\hline Bernard, M., 3, 4, 5 & Cross, P., 27 & Gagné, R.M., 50 \\
\hline Bertrand, Y., 13, 15, 18 & Crotty, T., 13, 71, 90, 115 & Garrison, R.D., 18 \\
\hline Boder, A., 132 & & Garth, R.Y., 61 \\
\hline Bohlin, R.M., 27 & D & Goodyear, P., 61 \\
\hline Bonk, C.J., 75, 111 & Damphousse, L., 77, 80, & Greenberg, S., 112 \\
\hline Boomer, G., 36 & $97,98,99,156$ & Gunawardena, C.N., 66 \\
\hline Bordeleau, P., 48 & Daniel, M.-F., 147 & Gutwin, C., 112, 116, \\
\hline Bouton, C., 61 & Darkenwald, G.G., 27 & 117 \\
\hline Briggs, L.J., 50 & Davie, L.E., 69, 71 & Guzdial, M., 61, 105 \\
\hline Brookfield, S., 27 & De la Teja, I., 63, 115 & \\
\hline Brown, A.L., 18, 81, 159 & Deschênes, A.J., 104, 105 & $\mathbf{H}$ \\
\hline Brown, J.S., 18 & Dick, W., 50 & Hamm, M.E., 31 \\
\hline Bruce, M.C., 71 & Dillenbourg, P., 76 & Hannafin, M., 133 \\
\hline Burge, E., 69, 71 & $\begin{array}{c}\text { Dimock, H.G., 80, 97, } \\
112,115,118,121\end{array}$ & $\begin{array}{l}\text { Harasim, L., 67, } 74 \\
\text { Harri-Augstein, S., } 17\end{array}$ \\
\hline C & Donovan, D.C., 69 & Hastie, R., 24 \\
\hline Carey, L., 50 & Doré, S., 48, 49 & Hatano, G., 23 \\
\hline Cash, D., 75 & Duning, B.S., 69 & Henderson, W., 13 \\
\hline
\end{tabular}


Henri, F., 88

Hill, F., 118

Hiltz, S.R., 67, 74, 88

Hoadley, C.M., 75, 105

Holoyak, K.J., 15

Hotte, R., 80

Hsi, S., 75, 105

\section{I-J}

Inagaki, K., 23

Jonassen, D., 13, 14, 21

Jonassen, D.H., 159, 160, 161,162

\section{K}

Kagan, S., 118

Kanselaar, G., 76, 86

Kenny, R.F., 110, 111

King, K.S., 75, 111

Klein, J.D., 31

Klemm, W.R., 75, 105, 107, 110

Knowles, M., 27

\section{L}

Lafortune, L., 147, 149

Laurillard, D., 61, 70, 132

Lave, J., 17, 24, 132, 159

Lebow, D., 28

Legendre, R., 97, 162

Lemke, J.L., 61

Lewin, I., 97

Lewis, R., 30

Li, T., 21

Linard, M., 61, 132

Lundgren-Cayrol, K., 35, $63,76,80,81,115,118$, $119,120,121,124,152$, 155

\section{M}

Mager, R.F., 50

Martineau, R., 148

Mason, R., 3, 7, 80
Mellar, H., 61

Miller, Gary E., 6

Mogensen, C., 76, 111

Morlan, John E., 47

Mullen, B., 83, 120, 121

$\mathbf{N}$

Newman, S.E., 18

$\mathbf{P}$

Palincsar, A.S., 18, 81, 159

Pallascio, R., 147, 149

Palumbo, D.B., 76

Paquette, G., 49, 50, 51

Paquin, C., 63, 109, 115, 156

Pea, R.D., 23, 24, 25

Pennington, N., 24

Perkins, D., 16

Pradl, G.M., 36

Prater, D.L., 76

Prescott, S., 76

Pugh, S.L., 70

$\mathbf{R}$

Ragan, T.J., 20

Reid, J.-A., 139

Resnick, L.B., 11

Ricciardi Rigault, C., 73, 85, 88, 156

Roberts, J.M., 71

Romainville, M., 149

Romiszowski, A.J., 50

Röscheisen, M., 76, 111

Rowntree, D., 61

\section{S}

Salomon, G., 16, 23, 70

Schön, D.A., 13

Schrage, M., 57, 60, 61, 76, 79, 112, 115

Schroeder, E.E., 110, 111
Shaw, M.E., 97

Shedletsky, L., 61

Smith, J., 15

Smith, P.L., 20

Snell, J.R., 76, 105, 107, 110

Snyder, L., 81

Spiro, R.J., 19, 20, 21

Spuck, D.W., 76

Stark, G., 112

St-Arnaud, Y., 80, 83, 97, $99,112,115,118,121$

Steeples, C., 61

Suthers, D., 61, 76

T

Tagg, A.C., 155

Tennyson, R.D., 81

Thomas, L., 17

Tomlinson, H., 13

Trollip, S.R., 50

Turoff, M., 88

V

Verduin, J.R., 27

von Glaserfeld, E., 14

\section{W}

Walker, D., 119, 121

Weiner, A., 61, 76

Wells, R., 69, 71, 90, 121

Welsh, J., 69

Wenger, E., 17, 132, 159

Wild, M., 160

Wilson, B.G., 10

Wilson, Brent, 17

Winn, W., 81

Winograd, T., 76, 111

Y

Yelon, S., 20 